\title{
Ecriture et Standardisation des Langues Gabonaises
}

Sous la direction de

Jacques Hubert \&

Paul Achille Mavoungou 
e. 



\section{Ecriture \\ et Standardisation des Langues Gabonaises}

Sous la direction de

Jacques Hubert \&

Paul Achille Mavoungou

Préface de J.T. KWENZI-MIKALA

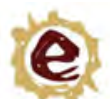

SUN PRESS 
Ecriture et Standardisation des Langues Gabonaises

Published by SUN MeDIA Stellenbosch

www.africansunmedia.co.za

www.sun-e-shop.co.za

All rights reserved. Copyright @ 2010 Jacques HUBERT \& Paul Achille MAVOUNGOU

No part of this book may be reproduced or transmitted in any form or by any electronic, photographic or mechanical means, including photocopying and recording on record, tape or laser disk, on microfilm, via the Internet, by e-mail, or by any other information storage and retrieval system, without prior written permission by the publisher.

First edition 2010

ISBN: 978-1-920109-90-5

e-ISBN: 978-1-920109-32-5

DOI: $10.18820 / 9781920109325$

Set in 10/12 Palatino Linotype

Typesetting by SUN MeDIA Stellenbosch

Cover design by SUN MeDIA Stellenbosch

Cover image by Petr Kratochvil - HTML: <a href="http://www.publicdomainpictures.net/viewimage.php?image=1786\&picture=colored-pencils" $>$ Colored Pencils $</ a>$

SUN PReSS is an imprint of AFRICAN SUN MeDIA (Pty) Ltd. Academic, professional and reference works are published under this imprint in print and electronic format. This publication may be ordered directly from www.sun-e-shop.co.za

Printed and bound by SUN MeDIA Stellenbosch, Ryneveld Street, Stellenbosch, 7600. 
La publication du présent ouvrage n'aurait pas été possible sans la contribution et le soutien des personnes ci-après:

- Pr. Rufus Gouws

- M. Augustin Tiwinot

- Mme Marina C. Nzalha, épouse Mavoungou.

- M. Hermano Ndenguino-Mpira

- Le Bureau du WAT

Puisse les uns et les autres trouver ici le témoignage de notre reconnaissance.

Nous sommes également très reconnaissants au Prof Jérôme T. Kwenzi-Mikala pour avoir accepté de préfacer le présent ouvrage.

Nous présentons enfin notre gratitude à Hugues Steve Ndinga-Koumba-Binza pour tout le travail de prospection et de coordination en vue de la publication de cet ouvrage. 



\section{SOMMAIRE}

Préface

i

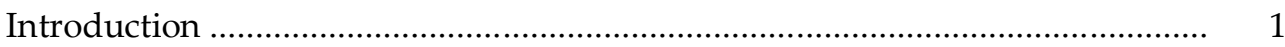

Etat des lieux sur 1'Enseignement des Langues Gabonaises ............................ 5 Jacques Hubert

Alphabet et Ecriture: approche historique et cas des langues gabonaises Hugues Steve Ndinga-Koumba-Binza

Alphabet et Orthographe: critères, qualités, conditions et vulgarisation Thierry Afane Otsaga

Orthographe, Standardisation et Confection des Dictionnaires Paul Achille Mavoungou

La Place des Tons dans l'Orthographe des Langues Gabonaises

Léandre Serge Soami

Unités-langues et Standardisation dans les langues Gabonaises Hugues Steve Ndinga-Koumba-Binza

Recommandations

Annexes

Index Thématiques 207

Les Contributeurs 209 



\section{PREFACE}

L'écriture et la standardisation des langues africaines en général et des langues gabonaises en particulier répondent à un idéal nouveau: annihiler les divers préconstruits sur l'orthographe de nos langues, et de la même manière, élargir et conserver les acquis de l'universalité. Pouvoir écrire et standardiser les langues gabonaises, c'est, à mon sens, unifier les différentes propositions d'alphabet de nos langues. C'est aussi réduire l'effet retors dont elles ont été l'objet. Harmoniser, enrichir, identifier les constantes des recherches réalisées et pouvoir enseigner ces acquis sans les galvauder sont les objectifs sur lesquels se sont fixés les auteurs du présent ouvrage.

Ecriture et Standardisation des Langues Gabonaises se présente comme un complément de réponse à un besoin réel : le développement et la transcription orthographique des langues gabonaises. Ce n'est certainement pas une chose négligeable que de parler d'écriture et de standardisation des langues gabonaises au moment où le monde se réduit progressivement en un village planétaire. En effet, le phénomène de mondialisation et les nouvelles technologies de l'information favorisent de plus en plus la maîtrise des langues des pays où sont élaborés ces nouveaux outils de développement, en l'occurrence le français et l'anglais. Il faudrait donc qu'un effort soit fait pour que nos langues puissent être utilisées dans les milieux scolaires, cadre d'apprentissage par excellence, et dans l'administration, moteur des échanges interculturels.

Le problème de nos langues, il faut aussi le souligner, commence lorsque les langues héritées de la colonisation (anglais, français et espagnol principalement), autrefois, facteurs de promotion sociale eurent à créer une classe d'évolués sachant faire usage des parlers européens. Cette classe aurait participé à l'élimination des langues locales dans les cellules familiales dont ils étaient les chefs. "L'éducation par le français rien que par le français ", peut on résumer la politique linguistique de cette époque. Les effets de cette politique sont manifestes de nos jours sur les jeunes, comme le fait remarquer Jacques Hubert dans son article.

Langues des technologies nouvelles, des échanges interculturels et des relations internationales, ces langues venues d'Europe participent de nos jours au processus de développement général de nos pays africains. Mais elles peuvent et doivent contribuer à l'épanouissement de nos langues. Il va falloir ainsi chercher à donner à nos langues le même statut technologique, culturel et sociopolitique sur le plan national que ces langues européennes que nous manions le plus souvent mieux que les nôtres. 
Ecriture et Standardisation des Langues Gabonaises ne saurait se réduire à une critique du colonialisme et de la léthargie, encore moins à une euphorie que dicte la nécessité de publication des chercheurs. C'est plus que des réponses dont peut avoir besoin le processus d'insertion des langues gabonaises dans le système éducatif. Pour ce faire, il faut systématiser, alphabétiser et orthographier les moyens de transmission desdits apprentissages. Je suis certain que cet ouvrage va contribuer efficacement à tous les processus de développement de nos langues : processus entamés depuis les travaux de Raponda-Walker jusqu'à l'adoption en 1999 d'un nouvel alphabet des langues gabonaises en Session de Concertation des experts. On peut retenir des différents chapitres que le développement et la conservation des langues gabonaises dépendent de l'écriture et de la standardisation. Ce qui, à mon avis, est juste puisque aucune langue ne s'est développée sans forme écrite ni norme.

Enrichir le débat scientifique sur la recherche linguistique au Gabon est également un objectif visé par le présent ouvrage. Il faut pour cela réduire les ambiguïtés définitionnelles. Un principe d'accord autour d'un alphabet communément adopté permettra de répondre aux "besoins énonciatifs et scripturaux des langues gabonaises". Il n'a donc pas été inutile de faire l'état des lieux. De cette connaissance du débat sur les langues gabonaises, les auteurs, chacun dans son domaine de compétence, donnent un avis dans une vision constructive. En effet, dans un maniement des concepts dont ils ont acquis l'art, ils critiquent, restaurent les faits jugés incompris et proposent des pistes de recherche et d'action.

Les auteurs nous le disent: "Science sans conscience n'est que ruine de l'âme". On osera nier que conscience sans science demeure tout de même une perte de l'âme. Engageons-nous donc à écrire, orthographier et standardiser les langues gabonaises. Laissons agir la symbiose synergique de la volonté qui vient de la jonction science et politique pour que se meuvent et se conservent ou que meurent les langues gabonaises. En définitive, je ne manquerai pas de relever que Ecriture et Standardisation des Langues Gabonaises est un excellent ouvrage qui remplit tous les critères de qualité et d'accessibilité dans une rigueur scientifique digne de spécialistes.

Qui sont les contributeurs de ce livre? Voici un point qui me touche également, et $q u$ 'il convient de relever. Tous des jeunes chercheurs qui ont su se faire encadrer par un homme rompu à la tâche et expérimenté dans le domaine. Il est sans conteste que Jacques Hubert a fait preuve de son souci pour le développement des langues gabonaises en initiant et faisant expérimenter la méthode appelée RAPIDOLANGUE pour l'apprentissage des langues gabonaises.

Cet homme a su voir les potentialités des jeunes que nous avons nous-mêmes formés et les conduire dans le cadre de ce bel ouvrage, étalant ainsi une relève bien assurée dans les métiers de la linguistique gabonaise. 
Enfin, pour ma part, Ecriture et Standardisation des Langues Gabonaises mérite d'être lu, et ses suggestions prises en compte pour sauvegarder les parlers gabonais.

Pr. Jérôme T. KWENZI-MiKala Coordinateur Général Chaire UNESCO Interculturalité Université Omar Bongo, Libreville 



\section{INTRODUCTION}

Ecriture et Standardisation des Langues Gabonaises a été conçu pour contribuer à la recherche des solutions à la problématique de l'alphabet et/ou de l'orthographe des langues gabonaises, et de manière générale à leur standardisation. La présente introduction aborde la situation de la linguistique gabonaise dans son rapport avec le développement des langues du Gabon. De nos jours, il est bien difficile de concevoir le développement des langues sans penser à l'écriture de celles-ci. C'est pourquoi, il est important de revenir ici sur les questions d'alphabet et/ou d'orthographe et de standardisation des langues du Gabon. La question du développement, au sens large du terme, des langues du Gabon est traitée avec un grand intérêt par de nombreux auteurs entre autres Kwenzi-Mikala et Idiata (voir les références bibliographiques de ces deux auteurs dans les différents chapitres du présent ouvrage). Nous recommandons la consultation de ces publications pour des informations complémentaires.

\section{LINGUISTIQUE GABONAISE ET DEVELOPPEMENT DES LANGUES}

La linguistique gabonaise est à un tournant crucial de son existence. Il est communément admis que la linguistique, au sens large, commence dans notre pays avec les travaux des missionnaires et des administrateurs coloniaux. Autrement dit, la linguistique gabonaise serait d'un âge largement au-delà de la cinquantaine si l'on part seulement de l'année d'indépendance, 1960.

Malgré cette relative tradition de recherche en linguistique, le développement des langues gabonaises achoppe encore sur des problèmes qui ont déjà trouvé des solutions dans bon nombre de pays africains. En effet les langues du Gabon ne sont ni standardisées, ni intégrées dans le système éducatif, et l'alphabet et/ou l'orthographe de ces langues ne sont pas encore adoptés et mis en application. En outre, le nombre de ces langues reste incertain, les grammaires d'un grand nombre d'entre elles ne sont pas encore élaborées, il n'y a pas encore de dictionnaires dans la majorité de ces langues et ceux qui existent ne sont pas toujours à la portée du grand public. Par conséquent, il est logique de déduire que le développement des langues gabonaises accuse un retard évident en comparaison avec la majorité des Etats africains.

Les raisons de ce retard sont diverses. La première est immanquablement la rupture qui s'est installée après l'accession du Gabon à la souveraineté internationale à la suite des travaux des missionnaires et administrateurs coloniaux. Même s'il est vrai que ces missionnaires et autres administrateurs 
coloniaux avaient peu ou pas du tout d'expérience en linguistique, la prise en compte et l'amélioration de leurs travaux auraient pu jouer un rôle important dans le processus de développement des langues gabonaises. La négligence de ces travaux pionniers favorise un perpétuel recommencement des recherches sur les aspects ayant déjà fait l'objet de propositions pertinentes. Cela est d'ailleurs illustré par la revalorisation à ce jour des travaux d'André Raponda-Walker ${ }^{1}$ qui servent aujourd'hui de référence à de nombreuses recherches sur les langues gabonaises.

Une autre raison de ce retard réside dans les orientations à donner à la recherche ${ }^{2}$ sur les langues gabonaises. A cela il faut ajouter la création tardive d'un département des sciences du langage et des unités de recherche linguistique (GRELACO, CRELL, Chaire UNESCO Interculturalité, etc.) au sein de l'Université Omar Bongo.

\section{POURQUOI CET OUVRAGE?}

Au-delà des raisons évoquées plus haut, le retard actuel du développement des langues gabonaises est aussi étroitement lié à l'absence d'un système d'écriture unifié3. En effet, les questions d'alphabet et/ou d'orthographe et de standardisation sont régulièrement évoquées lorsqu'il s'agit de parler du développement des langues du Gabon. Mais les propositions allant dans le sens de trouver des réponses à ce problème sont rares. Le présent ouvrage a pour objectif de proposer de nouvelles pistes de recherche susceptibles d'aider à la résolution des problèmes $\mathrm{d}^{\prime}$ alphabet et/ou d'orthographe et de standardisation des langues gabonaises.

Ecriture et Standardisation des Langues Gabonaises se présente en six chapitres chacun sous forme d'article individuel. Les contributeurs sont de formations diverses: pédagogue, lexicographes et phonéticien, mais ils sont avant de tous des linguistes qualifiés et soucieux du développement des langues gabonaises.

Dans son article, Jacques Hubert fait un état des lieux de l'enseignement des langues au Gabon. L'auteur insiste sur l'intégration des langues locales dans le système éducatif et fait le bilan des langues enseignées à titre expérimental dans quelques lycées et collèges de Libreville à l'aide de la méthode Rapidolangue

1 La majorité des travaux de Raponda-Walker sont aujourd'hui l'objet d'édition et réédition de la part de la fondation portant son nom (Fondation Raponda-Walker).

2 On note par exemple que la Fondation Raponda-Walker priorise la confection des manuels didactiques tandis que nombre de linguistes gabonais estiment que la description linguistique est l'étape préalable à tout développement des langues gabonaises.

3 Par alphabet unifié, on entend l'ensemble des graphèmes et symboles retenus pour l'écriture des langues gabonaises. Dans cet ensemble chaque langue puise et utilise les graphèmes et symboles dont elle a besoin pour sa transcription. Le présent ouvrage propose que les graphèmes et symboles retenus par la Session de Concertation sur 1'Orthographe des Langues Gabonaises de 1999 joue ce rôle d'alphabet unifié. 
élaborée avec le concours de la Fondation Raponda-Walker. Il revient également sur les conditions et les raisons de la conception de cette méthode. La question de l'orthographe et des sons pour l'écriture des langues gabonaises est également abordée. Jacques Hubert présente les critères de confection de l'orthographe proposée dans le Rapidolangue et sur son évolution en tenant compte des recommandations de la Session de Concertation sur l'Orthographe des Langues Gabonaises d'avril 1999.

Dans sa première contribution à ce volume, Hugues Steve Ndinga-Koumba-Binza fait brièvement l'historique du développement des alphabets des langues de manière générale. Il revient également sur l'écriture des langues africaines et fait des rapprochements entre celles-ci et les orthographes des langues européennes à alphabet latin. Il termine son étude en présentant l'état de connaissance sur l'écriture des langues gabonaises dont il fait une analyse des alphabets existants.

Thierry Afane Otsaga, dans son article, aborde les critères liés à l'élaboration de l'alphabet. Il part de l'expérience des pays des langues dites à longue tradition écrite pour proposer des voies qui pourront servir au choix d'un système unifié d'écriture pour les langues gabonaises. L'auteur insiste également sur le fait que tout système d'écriture est susceptible d'amélioration en fonction des besoins nouveaux. Par ailleurs, Thierry Afane Otsaga souligne que la promotion et la vulgarisation de ce système sont aussi importantes que le choix d'un système d'écriture unifié.

Dans son article, Paul Achille Mavoungou traite des problèmes d'orthographe, de standardisation et de confection de dictionnaires dans trois langues du Sud-Gabon, à savoir: le yilumbu, le yipunu et le civili. Selon l'auteur ces problèmes sont également liés à l'existence de variantes dialectales. Paul Achille Mavoungou fait entre autres une présentation critique des efforts entrepris par les missionnaires, administrateurs coloniaux et linguistes pour établir des conventions orthographiques dans les langues sus-mentionnées avant de proposer des conventions d'écriture et des faits de standardisation avec en filigrane la confection de dictionnaires dans lesdites langues.

Léandre Serge Soami quant à lui aborde le rôle et l'importance des tons dans le fonctionnement des langues gabonaises. Sa démarche est centrée sur le fonctionnement tonal des langues bantu en général et des langues gabonaises en particulier. Les analyses et décisions eu égard à la notation des tons pour les besoins de l'enseignement et du développement général de ces langues accordent une place de choix au grand public.

Dans sa seconde contribution, Hugues Steve Ndinga-Koumba-Binza propose une option pour la standardisation des langues du Gabon après avoir donné un aperçu de la situation dialectologique au Gabon et passé en revue les différents 
regroupements en unités-langues dont les langues du Gabon ont été l'objet. Il suggère l'identification des unités-langues et leur classification interne, telles qu'établies par Kwenzi-Mikala (voir l'article pour les références bibliographiques) comme point de départ du processus de standardisation en soutenant une coopération entre chercheurs et décideurs pour un choix quasi-arbitraire d'une variante dans chaque langue (ou unité-langue) comme norme que soutiendra la confection des dictionnaires et autres manuels didactiques.

Enfin, la présente publication ouvre de nombreux horizons et se veut informatif dans son approche. Il se propose également de fournir au public gabonais en général et aux locuteurs des langues concernées en particulier, des propositions de systèmes d'écriture. Il s'agit des graphèmes et règles d'écriture dont certaines langues gabonaises (le civili, le fang, le yilumbu et le yipunu) servent de support d'exemplification.

Jacques Hubert

Paul Achille Mavoungou

Hugues Steve Ndinga-Koumba-Binza 


\title{
ETAT DES LIEUX SUR L'ENSEIGNEMENT DES LANGUES GABONAISES: LE RAPIDOLANGUE ET L'ORTHOGRAPHE ${ }^{1}$
}

\author{
JACQUES HUBERT \\ FONDATION RAPONDA-WALKER, LIBREVILLE \\ (guerineau7@yahoo.fr)
}

\section{INTRODUCTION}

Parler de langues locales, de langues nationales, de langues régionales est un sujet d'actualité aussi bien au Gabon que partout ailleurs. Du fait de leur effet dominateur, les langues d'autorité (anglais, français, etc.) ont été pendant un temps perçues comme des moyens d'élévation sociale. Mais depuis la Conférence de Mexico (1982), il est communément retenu que la planète ne doit plus perdre de valeurs culturelles mais plutôt maintenir les écosystèmes culturels nationaux.

C'est ainsi que l'Union Européenne a autorisé les parlers régionaux et a demandé à tous ses partenaires de permettre leur apprentissage dans tous les pays de l'entité européenne. Elle a d'ailleurs créé en 1980, une "Grammaire de la Communication » (programme grammatical de la 6ème à la 3ème) et un «Vocabulaire lexical » (pour les mêmes classes). Mais nous savons aussi que la France, conforme à sa politique coloniale des temps passés, est toujours réticente à l'idée de promouvoir les parlers régionaux. Jusqu'à une date récente, des langues comme le breton, le basque, le gallois, l'occitan et le corse étaient interdits. Il en était de même du créole en Martinique. Le gouvernement Jospin en son temps avait amorcé l'apprentissage du corse sur l'île et nous connaissons les difficultés auxquelles il a eu à faire face.

Pourquoi une telle opposition? Parce que le colonisateur d'autrefois pensait que pour unifier un pays, il était indispensable que toute la population s'exprime dans une seule et même langue. Au fur et à mesure de leurs conquêtes, les Grecs, puis les Romains avaient imposé leur langue aux pays conquis, pour la même raison.

On donnait le fameux «symbole» aux enfants bretons qui parlaient en langue à l'école ; de la même manière, on l'a introduit en Afrique francophone, et par le fait

1 Une première version des données présentées ici a déjà fait l'objet de communications présentées par l'auteur au cours de divers ateliers et conférences. 
même au Gabon. Il ne s'agissait donc pas d'une politique nouvelle. Pour la France, imposer la langue française comme langue administrative était aussi un excellent moyen de recruter un personnel capable de communiquer dans la même langue que le colonisateur. Les peuples de la côte, les Kongo par exemple, puis les Mpongwè, n'hésitaient pas à envoyer dès le XVe siècle leurs enfants en Europe pour y apprendre le portugais ou l'espagnol, langue des explorateurs. Les rois de la côte atlantique eux-mêmes connaissaient les langues des puissances coloniales pour les avoir pratiquées pour les besoins de la cause.

Aujourd'hui, l'un des chevaux de bataille de la Francophonie est le développement des langues locales. Il en va de même pour l'Unesco qui l'encourage afin de lutter contre l'analphabétisme. Alors, pourquoi faut-il enseigner les langues locales ? Estil vrai que les langues sont en péril? Ce sont ici des questions auxquelles je voudrais donner une réponse en mettant un accent particulier sur la méthode Rapidolangue et sur le problème de l'orthographe.

\section{POURQUOI FAUT-IL ENSEIGNER LES LANGUES LOCALES ?}

Au Gabon, d'après les statistiques du début de l'année 2001, 73 \% de la population gabonaise habite dans les villes et les enfants parlent majoritairement la langue française et ne connaissent plus leur langue maternelle. S'il existe un phénomène de rejet des langues nationales par certains jeunes nés depuis 1975, il n'en demeure pas moins que d'autres commencent à comprendre l'importance de leur langue et lance déjà l'anathème sur leurs parents qu'ils interpellent de la manière suivante : «Pourquoi est-ce que nous ne parlons pas notre langue »? Cet appel doit être entendu pour des raisons toutes universelles, et parce que les langues en elles-mêmes en éprouvent le besoin.

Quinze raisons en faveur de l'enseignement des langues:

1. La raison primordiale de cet enseignement est la disparition rapide et progressive de nos langues, n'en déplaise à certains intellectuels qui affirment le contraire. Arrivé en 1966 au Gabon, j'ai déjà assisté à la disparition de fait du séké et du benga dans les environs de Libreville. Les peuples sékyani et benga sont linguistiquement assimilés aux Ngwè-Myènè. Evolution irréversible. Ainsi, pour le fang, on ne dit plus «bëkalë » mais «békalé », etc.

2. Deuxième raison: "Un peuple sans culture est un peuple sans âme ». La langue est le meilleur véhicule de la culture. D’où la nécessité de soutenir les langues nationales et de faire le nécessaire pour leur apprentissage.

3. L'enseignement des langues est devenu le leitmotiv des principaux pays africains, l'une de leurs plus grandes préoccupations. Ce n'est pas un phénomène de mode, mais une excellente manière de manifester son identité. 
Hors de son pays, un Africain reste Africain de par son comportement. Il transpire son origine et ne peut nier son identité. Il se sent mal à l'aise à l'étranger s'il ne connaît pas sa propre culture. Voilà pourquoi les pays africains ont redécouvert la nécessité de favoriser le développement des langues nationales. D'autres raisons s'ajoutent à ces trois raisons fondamentales.

4. Enseigner les langues pour lutter contre l'analphabétisme. Cette raison reste encore valable pour certaines zones rurales où les personnes ne s'expriment que dans leurs langues.

5. Les langues maternelles permettent encore de communiquer avec les autres. Une langue n'arrive pas à s'imposer s'il n'y a pas une nécessité pour cela. La connaissance de la langue des autochtones favorise l'échange et le commerce. $C^{\prime}$ est de cette manière que dans la sous-région, le lingala a progressé et continue de s'étendre vers les frontières gabonaises après avoir franchi le fleuve Congo. Un certain nombre de chants religieux ont déjà introduit le lingala dans la liturgie au Gabon.

6. La menace de la langue française est indéniable. L'expansion des médias modernes est un très grand danger pour les langues locales et l'impact de la langue française véhiculée par la radio et la télévision a abouti au fait que même les enfants de moins de cinq ans parlent le français sans aucune gêne d'autant que certains parents eux-mêmes ne parlent plus leur langue maternelle à la maison, en particulier les couples mixtes (ex. un père fang, une mère myènè, etc.). Certains écrivains francophones l'avaient bien compris puisque Makhily Gassama (ancien ministre de la Culture au Sénégal, ancien Directeur Général de l’UNESCO au Gabon), déclarait que «la langue française peut aider à la circulation et la promotion de nos langues ».

7. C'est également un problème philosophique. En effet, le refus des anciens de transmettre leur savoir et de favoriser l'identité culturelle de leur peuple à travers us et coutumes, sous prétexte de préserver leurs secrets est à l'origine de la perte de la philosophie traditionnelle qui s'exprime généralement à travers rites et croyances. C'est pourquoi « l'intelligentsia » africaine a relevé la tête pour le faire comprendre.

8. C'est un problème scientifique. L'expérience a montré que le développement de la pensée passe par l'éveil de l'esprit scientifique. A une époque où nous dévorons tout ce qui est moderne et technologique, il est important de comprendre que cet esprit scientifique se développe chez les enfants par le jeu et dans n'importe quelle langue. Les colonisateurs ne disaient-ils pas que le vocabulaire de nos langues est insuffisant pour un enseignement à partir des langues vernaculaires ? On sait qu'il est possible d'enseigner dans les langues locales les notions mathématiques et scientifiques. Nous en avons une preuve avec les dictionnaires d'André Raponda-Walker : 35000 mots pour la langue omyènè, 16000 mots pour la langue ghetsöghö et 10000 pour la langue gisir. Il 
reste cependant aux scientifiques de prouver que c'est réellement possible et qu'il n'est pas trop tard pour le faire.

9. L'apprentissage d'une langue étrangère est plus facile si les mécanismes de la langue maternelle sont connus. Ceci était encore valable pour le Gabon il y a vingt ans, mais ne l'est plus aujourd'hui, suite à l'invasion de la langue française sur tout le territoire. Si au début de l'indépendance on condamnait le colonisateur pour avoir imposé sa langue, on se rend compte désormais de la nécessité de maîtriser une langue à vocation mondiale, telles que l'anglais, le français, l'espagnol ou l'allemand. Il n'y a plus que les «frustrés » de la colonisation qui continuent à stigmatiser cette incursion étrangère dans leur vie. Les écrivains africains d'ailleurs reconnaissent que la connaissance de la langue française, par exemple, leur a permis de propager leur propre culture à travers notamment les romans dits « engagés ».

10. Une autre raison essentielle est celle de permettre aux peuples de sauver les valeurs culturelles de leurs pays par la sauvegarde de leurs langues. Il est fréquent de constater qu'un sentiment d'infériorité naît chez les peuples qui ont abandonné leurs langues maternelles, car un membre de leur communauté se sent écrasé, à l'étranger, par des langues de prestige - l'anglais par exemple - lorsqu'il voyage ou vit dans un autre pays. C'est d'ailleurs le cas des Francophones face à la propagation de la langue anglaise dans le monde. C'est aussi l'une des raisons du succès du concept de la « Francophonie ». C'est pour le retour à la dignité et à une certaine confiance en soi que l'apprentissage des langues vernaculaires doit être introduit dans le système éducatif.

11. L'obtention de meilleurs résultats scolaires si la langue maternelle est pratiquée. Mais il est indispensable pour cela que la langue maternelle soit maîtrisée. Les enseignants savent les difficultés qu'ils rencontrent dans l'enseignement des pronoms personnels. Exemple : "Je l'ai dit de venir » au lieu de «Je lui ai dit de venir »... «Je les ai données (des oranges, pour : je leur en ai données).

12. Pour un notable, il est politiquement correct de se faire comprendre dans sa langue. Sur le plan politique, la connaissance de la langue maternelle est valorisante auprès des populations: "C'est un vrai fils du pays ». Nous nous rappelons la réaction de populations villageoises lorsque l'un de leurs députés a été obligé d'avouer qu'il ne maîtrisait plus sa langue maternelle. C'est Gudschinsky (1973) qui considère un groupe comme lettré lorsqu'il est capable de parler et d'écrire dans sa propre langue.

13. L'éducation en langue traditionnelle est toujours efficace. Nous savons que les néophytes reçoivent toujours les enseignements et la connaissance de leur peuple dans la langue pendant les initiations. Ajoutons avec l'écrivain malien Abdulaye Ascofare: «le développement d'un pays passe par l'enseignement des langues maternelles ». 
14. L'écriture des langues maternelles est le moyen de redonner confiance à tout un peuple. Si les missionnaires, puis l'administration coloniale ont contribué au développement de l'étude de la langue française dans nos régions, il faut reconnaître que les missionnaires sont les seuls à avoir donné des enseignements de la Bible et du catéchisme en langue. Ils ont été les premiers à les écrire. Il est important ici de rendre hommage à Charles Sacleux, Cssp. - à la suite de quelques pionniers - qui a le premier institutionnalisé les principes de l'orthographe des langues africaines. Mgr Raponda-Walker avait eu connaissance des règles édictées par Sacleux dans son livre Essai de Phonétique (1905) puisque l'on a retrouvé trois ouvrages de Sacleux dans les archives de Sainte-Marie. C'est à partir de ces principes qu'il a lui-même proposé son Alphabet des idiomes gabonais en 1932 (Journal de la Société des Africanistes T II, fascicule 2)

15. La culture du nationalisme à travers le multilinguisme. Contrairement aux idées reçues, la connaissance de plusieurs langues ne s'oppose pas à l'unité nationale. Le monolinguisme est une illusion et, en fait, une erreur historique monstrueuse. Le kinyarwanda, même langue pour les Tutsi et les Hutu, n'a pas empêché le génocide. C'est une infirmité aujourd'hui de n'avoir qu'une seule langue. A l'opposé, le plurilinguisme favorise une meilleure communication entre les peuples et évite des tensions. C'est de cette manière que l'on reconnaît au Gabon que des ethnies différentes se comprennent et utilisent le même langage.

Après avoir exposé les principales raisons qui nous recommandent l'apprentissage des langues vernaculaires, voyons ce qui se fait au Gabon.

\section{LES LANGUES GABONAISES ET LEUR APPRENTISSAGE}

Il est heureux de constater que le Gabon s'est lancé dans une véritable politique linguistique depuis les Etats Généraux de l'Education de 1983. Ce mouvement devrait être irréversible et, comme évoqué plus haut, tous les pays africains en sont conscients.

En 1979, la CONFEMEN2 organisait un colloque sur la « revalorisation et l'intégration des langues nationales dans les systèmes éducatifs » (Dodo-Bounguendza 1998/1999).

La publication d'un Alphabet scientifique des langues du Gabon en 1990 fut un progrès énorme pour une meilleure connaissance de nos langues et le travail des linguistes ne fait que commencer.

2 La Conférence de Ministères de l'Education Nationale (CONFEMEN). 
Les chercheurs de l'Université Omar Bongo apportent également leur contribution à l'inventaire des parlers nationaux (Kwenzi-Mikala 1987, 1990 et 1998). On trouve cette liste dans Langues du Gabon édité par la Fondation Raponda-Walker en 1998. Plusieurs centres de recherche se créent dans différents domaines des sciences humaines (sciences du langage, histoire, anthropologie, etc.). Ceux-ci souhaitent tous l'intégration des langues locales dans le système éducatif. Il y a par exemple le LUTO-DC (Laboratoire Universitaire de la Tradition Orale et des Dynamiques Contemporaines), le LASCIDYL (Laboratoire des sciences humaines et de la dynamique du langage), le GRELACO (Groupe de recherche en langues et cultures orales), le CRELL (Centre de recherche en langues et linguistique), le NDAGA (Centre pour l'étude des langues gabonaises) et la Chaire UNESCO Interculturalité pour ne citer que ceux-là.

A l'exception faite du NDAGA et de la Chaire UNESCO Interculturalité, toutes ces structures de recherche ont vu le jour avant 1995 lorsqu'a émergé d'un grand silence le S.O.S. de la Fondation Raponda-Walker sur la sauvegarde de nos langues. Ce S.O.S sera suivi du lancement de l'apprentissage des langues gabonaises dans plusieurs établissements de la capitale et de l'intérieur du pays à la rentrée 1996-1997, à la suite de l'expérience tentée en 1995-1996 à l'Institution Immaculée Conception. Dans le même ordre d'idées, un concours sur les langues nationales est organisé au Lycée Djoué Dabany sous la supervision de la Fondation Raponda Walker, de février 1998 à février 1999.

Par ailleurs, il faut noter qu'entre-temps un poste de "Conseiller technique, chargé de l'intégration des langues nationales dans le système éducatif » a été créé au Ministère de l'Education Nationale en 1997. Au cours de la même période, une table ronde a été organisée sur les Recherches linguistiques et l'enseignement des langues gabonaises (10/12/97).

En outre, l'Ecole Normale Supérieure (ENS) a initié en 1999 un cycle CAPES en langues nationales pour la formation des futurs professeurs dans cette discipline. Une initiation à l'enseignement des langues gabonaises est prévue pour les conseillers pédagogiques et les professeurs-adjoints d'école.

La même tendance s'observe à l'Institut Pédagogique National (IPN) qui a ouvert un Département des Langues Nationales. Ce département est sensé dans un proche avenir se muer en une Direction des Langues Nationales. A l'heure actuelle, plusieurs formateurs de formateurs y sont affectés.

A l'Université Omar Bongo (UOB) encore, une filière "langues nationales » a été créée au Département des Sciences du Langage (DSL). Les étudiants de cette filière sont supposés intégrer, à la fin de leur année de maîtrise le cycle CAPES en langues nationales à l'ENS ou encore le Département des langues nationales de l'IPN.

3 Certificat d'Aptitude Professionnelle à l'Enseignement Secondaire (CAPES). 
Des études sont entreprises tous azimuts en sociolinguistique. Il s'agit d'études comparatives sur les noms d'animaux, de poissons, sur la tonalité, les classes nominales, etc. L'activité linguistique est indéniable et les problèmes analysés, triturés. Que l'on me pardonne les nombreux oublis en la matière tant les personnes intéressées par le nouveau phénomène des langues sont nombreuses, aussi bien des linguistes que des profanes amoureux de leurs langues maternelles!

En ce qui concerne le système d'écriture, une session de concertation des experts de l'Enseignement Supérieur, de l'Education Nationale, de la Société Internationale de Linguistique (SIL International) et de la Fondation Raponda-Walker s'est tenue en avril 1999 pour fixer les règles d'écriture des langues gabonaises.

Il faut aussi rendre justice à la radio et à la télévision nationale d'une part ainsi qu'aux radios périphériques d'autre part, qui sensibilisent - à travers des émissions très variées et des concours - la population sur l'importance de connaître et de parler en langue maternelle. L'émission Le polyglotte de la radio nationale, entre autres, est particulièrement prisée.

Revenons aux recommandations de l'UNESCO et de la FRANCOPHONIE sur les langues vernaculaires. Ces deux organismes en ont fait leur cheval de bataille comme nous l'avons dit ci-dessus et l'organisation de colloques sur le sujet en est la preuve. Le programme LINGUAPAX de l'UNESCO prône l'organisation de séminaires pratiques sur l'Aménagement linguistique au Gabon, la Réalisation et la conception de manuels didactiques en langues nationales. La Banque Mondiale se propose aussi de promouvoir les langues à traditions orales.

Il est évident qu'il s'agit d'un profond revirement de mentalité à travers le monde entier et les dirigeants africains l'ont parfaitement compris. Pour le Gabon, l'on peut voir comme je viens de le présenter, que les potentialités institutionnelles et humaines sont déjà à pied d'œuvre pour l'enseignement des langues locales dans un délai à court terme. Mais un problème qui semblait mineur est devenu la principale pierre d'achoppement au lancement de l'apprentissage des langues dans l'ensemble du système éducatif.

\section{LE BLOCAGE A L'ENSEIGNEMENT DES LANGUES}

Le blocage à l'introduction des langues bantu dans le système éducatif se résume en grande partie au défaut d'un alphabet et d'un système d'écriture adéquats, si l'on ne tient pas compte de la volonté politique qui est souvent lente à la décision.

De fait, l'acharnement de certains linguistes africains de nombreuses universités bantu a provoqué un tel blocage que plusieurs pays ont été incapables de résoudre le problème des alphabets et des orthographes de leurs langues en raison principalement de l'importance accordée à l'écriture scientifique et à la tonalité. 
Pour eux, il est clair que les langues bantu sont des langues à tons et qu'il faut impérativement noter les tons. Les exemples des universités ayant imposé un tel diktat ont fait en sorte qu'un pays comme le Centrafrique où le sango est une langue nationale - au plein sens du terme - parlée dans tout le pays, n'est ni enseignée ni écrite (décret $n^{\circ} 84025$ du 28 janvier 1984 fixant l'orthographe officielle du sango - langue nationale centrafricaine -). C'est le cas en République du Congo (Brazzaville) où les universitaires reconnaissent eux-mêmes ce blocage.

Réservons donc l'écriture scientifique aux scientifiques, c'est-à-dire aux chercheurs, aux linguistes, aux universitaires et à leurs étudiants. Mais une telle écriture ne peut, en aucun cas, se justifier pour le commun des mortels. La réflexion engagée sur le sujet permet de conclure de la manière suivante:

- Sachant qu'une langue s'apprend par imitation (cf. Circulaires officielles sur l'enseignement des langues vivantes);

- Considérant que la connaissance des tons est importante.

- Vu l'expansion des langues nationales dans les pays où l'écriture des tons est facultative, il convient de savoir:

- que les tons grammaticaux ne sont pas stables et peuvent prendre une place variable sur un mot;

- que l'absence de tons n'a jamais empêché un apprenant d'étudier une langue;

- que l'on s'accorde aujourd'hui, dans plusieurs universités, pour dire que l'écriture de la tonalité est plus qu'une surcharge et peut même devenir un phénomène de rejet;

- que les moyens financiers n'autorisent que rarement la prise en compte des tons, en particulier lorsqu'il s'agit de la confection d'un journal en langue.

Il importe de n'écrire les tons que s'ils sont indispensables à la compréhension du vocabulaire. Cela ne dispense pas l'enseignant de les connaître. Rendons ici hommage au LUTO-DC qui a proposé une écriture simplifiée pour les symboles de l'ASG (Alphabet Scientifique du Gabon). Nous savons aussi que l'instruction ministérielle $\mathrm{n}^{\circ}$ 13.02/03.2/003 du 2 juillet 1985 portant fixation de l'orthographe officielle du Kinyarwanda, après avoir revu les instructions du 6 février 1974, en son article 24, réserve la tonalité, représentée par l'accent circonflexe pour le ton haut et l'absence de signe diacritique pour le ton bas, à l'usage scientifique exclusivement. Nous savons également qu'au Rwanda, l'enseignement se fait, à égalité de plage horaire, en kinyarwanda, français et anglais (6 h par discipline et par matière, dès l'entrée en classe de 1ère, équivalente à notre sixième). C'est la raison pour laquelle la langue maternelle est parlée et écrite dans tout le pays tout comme au Burundi.

$C^{\prime}$ est en effet, à ce prix que les langues nationales se développeront et sauveront les valeurs culturelles de nos pays. Ajoutons qu'une commission interministérielle a 
été mise en place depuis février 1997, par le Premier Ministre, Chef du Gouvernement.

Par ailleurs, le Ministère de l'Education Nationale envisage de prendre en compte toute langue dominante régionale si elle dispose d'un bon dictionnaire et d'une bonne grammaire, et de l'enseigner selon les zones définies ci-après : pour le Centre (Lambaréné), le Sud (Tchibanga, Mouila), l’Est (Koulamoutou, Franceville) et le Nord (Oyem et Makokou).

\section{LA METHODE RAPIDOLANGUE ET SON BILAN}

\section{HISTORIQUE ET MOTIVATIONS}

La méthode d'apprentissage des langues RAPIDOLANGUE de la Collection RAPIDO est née d'une nécessité. Initié par la directrice de l'Immaculée Conception en 1995, l'enseignement des langues locales ne possédait aucun appui didactique approprié et les professeurs éprouvaient beaucoup de difficultés à lancer un programme ainsi mal défini.

Le point de départ de RAPIDOLANGUE remonte au changement radical de l'enseignement des Langues naturelles vivantes en 1962 après la publication par le Ministère de l'Education Nationale de France de nouvelles instructions officielles relatives à cet enseignement: faire parler des langues étrangères. En un mot, substituer à l'ECRIT un enseignement ORAL pour permettre aux apprenants de parler correctement une langue étrangère - anglais, allemand, espagnol et même latin - à la fin des études secondaires. C'est de là que sont nées les méthodes audiovisuelles de l'apprentissage des langues, quelque 15 ans plus tard et la British Broadcasting Corporation (BBC) y est pour beaucoup dans ce succès. Des méthodes intensives voient le jour : en 300 heures vous maîtrisez une langue, ce qui correspond à un bain linguistique de 3 mois ou à un apprentissage échelonné de 3 heures par semaine dans nos classes du secondaire pendant 4 ans (à raison de 25 semaines effectives par an). Un élève de la classe de 3ème est censé parler en langue à la fin de son premier cycle.

Est-il inutile de faire remarquer que le CLAD (Centre de Linguistique Appliquée de Dakar) est également né en 1962 ?

C'est le linguiste britannique Crystal (2000) qui lançait il y a quelques années un vibrant appel pour la protection de ce précieux patrimoine culturel. Il disait que dans cent ans, la moitié des 6.000 langues de la planète auraient disparu. Un éminent linguiste africain proclame de son côté que les langues qui ont moins d'un million de locuteurs auront également disparu dans les vingt ans. Et nous savons évidemment que c'est le cas pour nos langues. 
C'est pour cette raison que, face à l'inertie qui règne au Gabon, malgré l'intervention des Etats Généraux de l'Education Nationale en 1983 qui prônaient l'introduction des langues dans le système éducatif à la suite du Colloque de Yaoundé, la Fondation Raponda-Walker lance son fameux S.O.S. le 24 mars 1995, pour tenter de stopper à sa manière, la disparition de nos langues au Gabon. Dans la foulée, elle produit en un an et demi, un manuel d'apprentissage, à la demande de la directrice de l'Immaculée Conception, Sœur Izabete, dans lequel on introduit d'abord cinq langues parmi les plus parlées sur tout le territoire national et un an plus tard, un autre manuel avec deux autres langues. Aujourd'hui, neuf langues sont prêtes pour l'enseignement. Il s'agit, par ordre alphabétique, des langues suivantes: le fang, le ghetsöghö, le gisir, l'ikota, le yinzebi, le lembaama (pour l'obamba), l'omyènè, le vili et le yipunu.

La méthode RAPIDOLANGUE peut s'appliquer à toutes les autres langues du pays.

\section{PRINCIPES ORIGINELS D’UNE METHODE D'ENSEIGNEMENT DE LANGUE}

AFRICAINE

1. Une méthode s'apprend par imitation, répétition et mémorisation (cf. Circulaires officielles sur l'enseignement des langues vivantes) et non par l'écrit.

2. Une écriture accessible à l'homme de la rue est indispensable pour éviter un phénomène de rejet, aussi bien à l'élève qu'aux enseignants qui dans leur majorité n'ont pas une formation de linguiste.

3. L'écriture des tons n'est pas une condition sine qua non pour l'apprentissage d'une langue, même d'une langue à tons (ex. le kinyarwanda, le kirundi, le swahili...).

4. L'écriture de tous les tons constitue une surcharge pour la saisie et la lecture. Comme il a été dit plus haut, ils varient dans les tons grammaticaux ou selon l'intonation du locuteur

5. La parution de journaux en langues constitue un objectif très louable

6. L'importance des coûts financiers, en particulier des frais d'impression. C'est pourquoi la méthode cible les élèves du secondaire plutôt que ceux du primaire. Au départ, la Fondation a voulu montrer que les langues maternelles, en déperdition, pouvaient s'enseigner comme n'importe quelle langue étrangère : anglais en particulier.

7. L'économie de temps dans la saisie (écriture possible sur n'importe quelle machine à écrire).

8. Le manque d'enseignants. Pour un enseignement national, il est impossible, de former dans l'immédiat, des linguistes rompus aux techniques de l'apprentissage des langues bantu. Mais il est indispensable de donner des notions pédagogiques aux volontaires maîtrisant leur propre parler. 


\section{Enfin, la Fondation n'a pas cherché à faire ouvre scientifique.}

Tous ces points ont été abordés à l'assemblée générale de juin 1995, à l'origine du manuel que nous connaissons. Le feu vert a été donné.

\section{LA FONDATION PART DONC SUR LE TERRAIN POUR:}

1. savoir quelles langues proposer. Les études ont été menées principalement au nord et à l'est et se sont basées sur les résultats du recensement de 1993 par le Ministère du Plan. Le problème des villes a été abordé.

2. trouver des traducteurs acceptant d'entrer dans le projet. Le problème a été ardu.: pas de volontaires en gisir, teke, vili... La question d'écriture a provoqué des réticences sérieuses. La simplification et l'universalité proposées par le Fondation ont suscité un réel intérêt chez les traducteurs. De plus de vingt volontaires au départ, nous nous sommes retrouvés finalement à treize.

\section{PRINCIPES DE BASE DE LA METHODE RAPIDOLANGUE:}

1. C'est une méthode audiovisuelle.

2. Elle utilise le vocabulaire fondamental basé sur la fréquence des mots : 1500 mots de base pour les classes de $6^{\mathrm{e}}$ et $5^{\mathrm{e}}$.

3. Pas de grammaire traditionnelle. Utilisation de la grammaire de communication (voir textes de l'Union Européenne en la matière) ; c'est-à-dire que la grammaire y est abordée de manière ponctuelle et utile, laissant au niveau 2 (intermédiaire) et au niveau 3 (avancé) le soin d'introduire l'étude systématique de la grammaire.

4. Utilisation de la grammaire structurale par les exercices de répétition, substitution et transformation.

5. Mémorisation des textes, pour la plupart sous forme de dialogues ou de conversations. C'est le meilleur moyen de générer les automatismes indispensables à une première conversation.

6. Enregistrement de cassettes pour les apprenants solitaires

7. L'écriture scientifique et les tons sont réservés au niveau universitaire (voir comme il a été dit, l'instruction ministérielle $\mathrm{n}^{\circ}$ 13.02/03.2/003 du 2 juillet 1985 portant fixation de l'orthographe officielle du Kinyarwanda, qui, après avoir revu les instructions du 6 février 1974, en son article 24, réserve la tonalité, représentée par l'accent circonflexe pour le ton haut et l'absence de signe pour le ton bas, à l'usage scientifique exclusivement)

8. Pas de panachage pour l'enseignement (mélange langue et français)

9. Alphabet applicable à toutes les langues du Gabon (tout en acceptant les signes diacritiques inévitables là où c'est nécessaire)

10. Privilégier la langue courante et l'équivalent, à une traduction littéraire.

11. Faire des dictées de contrôle à partir des textes étudiés. 
12. Préparer un lexique pour chaque langue.

13. Enfin, amener l'apprenant à penser et pourquoi pas « à rêver dans sa langue ».

Compte tenu des principes ci-dessus, la Fondation Raponda-Walker a proposé la méthode suivante d'écriture:

1. Utilisation de l'alphabet latin chaque fois que c'est possible car les apprenants sont familiers de cet alphabet. Chaque lettre y conserve sa valeur, sauf le « $u$ » qui se prononce $[u]$ et le «e $»$ qui se prononce $[e]$.

2. La lettre «c» de l'alphabet latin prête à confusion avec de nombreuses écritures et principalement avec le [t $\mathrm{t}]$ de l'Alphabet Phonétique International (API). Elle est remplacée par la lettre « $\mathrm{k} »$

3. Il en est de même de la lettre « $\mathrm{x} »$; en API [x]. Nous avons adopté l'écriture régulièrement admise en pratique $\mathrm{du}$ « gh » pour traduire le son API [x].

\section{PRINCIPES DE L'ELABORATION DE LA « COLLECTION RAPIDO »}

Les manuels d'apprentissage de la méthode RAPIDOLANGUE ont ciblé, en premier lieu, les élèves du Premier Cycle du Secondaire et sont utilisés dans les classes correspondantes depuis 1996 dans plusieurs établissements de Libreville, à Port-Gentil et à Koulamoutou.

- Cette méthode a fait appel aux dernières connaissances en matière de l'enseignement des langues vivantes et dispose de cassettes à l'usage des apprenants à domicile.

- Elle obéit aux instructions officielles des Ministères de l'Education Nationale pour les langues vivantes. Est-il besoin de rappeler quelques-unes de ces règles?

a. L'objet de l'enseignement des langues vivantes est d'apprendre, dès le début, à parler, puis à lire et écrire la langue d'aujourd'hui ; et à exprimer ORALEMENT, les faits et les idées de la vie la plus générale. Tel est l'objectif à ne jamais perdre de vue. En conséquence, tous les exercices doivent privilégier la conversation.

b. Une langue vivante s'apprend par IMITATION. L'oral précède l'écrit comme l'exemple précède la règle. L'élève imite le maître (ou la cassette) et ceci, plus spécialement dans les classes de «débutants », ce qui correspond aux niveaux 1 et 2 ( $6^{\mathrm{e}}$ et $5^{\mathrm{e}}$ de nos classes).

c. Pas d'exposés ni de conférences durant les cours mais des exercices de conversation.

d. Le rôle du professeur est de créer et de maintenir des automatismes. 
e. L'appui de la mémoire est primordial. Tout doit être immédiatement accessible et oralement disponible dans la mémoire (cf Instructions Officielles).

f. Toutes les conversations doivent respecter le naturel de l'intonation.

g. Au-delà de ces principes généraux, des principes techniques ont présidé à l'élaboration des manuels:

h. une écriture simple accessible à l'homme de la rue

i. une même écriture pour un même son, dans toutes les langues du Gabon. Les sons pertinents des langues gabonaises retenues ont d'abord été inventoriés.

j. utilisation de la grammaire de la communication

Finalement, l'élaboration du manuel RAPIDOLANGUE a tenu compte des principes ci-dessus et des règles scientifiques ci-après:

- les manuels de niveaux 1 et 2 utilisent le principe de la fréquence du vocabulaire, c'est-à-dire qu'ils proposent l'étude du VOCABULAIRE FONDAMENTAL (Lexique de 1500 mots en deux ans).

- les nouveaux manuels proposent l'orthographe adoptée par la session de concertation sur l'orthographe des langues gabonaises des experts de l'Enseignement Supérieur, de l'Education Nationale et la Commission Nationale de 1'UNESCO réunis en colloque les 8-10 avril 1999.

- La grammaire s'apprend à partir d'exercices structuraux de répétition, de substitution et de transformation (méthode RST), conformément aux nouvelles dispositions de l'enseignement de la grammaire de la communication (83 exercices de langage dans le manuel du niveau 1, et 118 dans celui du niveau 2). RAPIDOLANGUE n'est donc pas un manuel pour touristes comme le laissent entendre des étudiants qui ne l'ont pas appliqué ou l'ont mal utilisé, par méconnaissance de la Grammaire Structurale.

- Le vocabulaire est appris à partir du vocabulaire de base, allant du simple au composé et la grammaire d'une manière progressive.

- L'écriture a été régie par les principes de simplicité et fonctionnalité ; et les signes diacritiques ont adopté la solution déjà proposée par le LUTO (Laboratoire Universitaire de la Tradition Orale) et la commission ad hoc susmentionnée, à savoir le soulignement, afin de préserver l'écriture des tons si nécessaire (ex $: \underline{e}=[\varepsilon]$ au lieu de /è/ qui porte une autre signification)

- Le principe de l'écriture des tons adopté privilégie les tons hauts (accent aigu) indispensables à la compréhension et ne gêne pas les signes. Les tons bas (accent grave) ou moyens pourront être mentionnés pour la clarté de la compréhension.

- La longueur vocalique est représentée par le redoublement de la voyelle (ex. lembaama) 
- L'adoption $d u g h$ commun à bon nombre des langues gabonaises pour le son contesté $d u[x]$ (ex. [xalam]: nom d'une danse célèbre au Sénégal). Nous écrirons donc «mvöghe» ou [mvoghə] en langue fang et non [mvóxé]; "ghetsöghö » au lieu de "xetsóxó », etc. Pour les mêmes raisons, nous évitons l'écriture du [d3], toujours mal prononcé comme dans Banjul, la capitale de Gambie et le [t $\mathrm{t}]$ qui prête à confusion et nous préférons le ny pour 'nyama' [nama] au lieu de 'gnama'.

\section{LA METHODE DANS SON APPLICATION}

La méthode RAPIDOLANGUE est donc enseignée, à titre expérimental, dans plusieurs établissements privés. Elle a fait ses preuves depuis 1996, et de nombreuses améliorations y ont été apportées, suite aux recommandations du Ministère de l'Education Nationale. Efficace, elle l'est si l'enseignant applique les conseils qui lui sont prodigués et utilise à bon escient la mémorisation et les exercices structuraux. Elle couvre actuellement les classes de débutants (6e et $5^{\mathrm{e}}$ en premier cycle) et le manuel de niveau III est à l'étude.

Une réflexion sur son élargissement dans deux ou trois établissements publics de Libreville est menée au Cabinet du Ministre de l'Education Nationale et son application a commencé depuis deux ans. De futurs professeurs de l'Ecole Normale Supérieure se préparent à l'inspection des enseignants en langues. Une heure de formation pédagogique a été dispensée par la Fondation Raponda-Walker aux enseignants en langues gabonaises de Libreville, une fois par mois. Ceci a pris effet dès l'année scolaire 2000-2001.

Des Modules éducatifs en faveur des enseignants en langues nationales se sont tenus à Port-Gentil et à Koulamoutou.

Enfin, un BEPC blanc a été organisé dans plusieurs lycées en avril 2001 et des soirées culturelles ont répondu à l'engouement et au succès de celles des années précédentes. Revalorisant les langues nationales, elles se sont déroulées en présence des parents et des amis des élèves.

\section{DES RESULTATS DE LA METHODE}

\section{QUELLES LANGUES SONT ENSEIGNEES ?}

Après enquête appropriée, le choix de la Fondation s'est porté sur les langues les plus parlées (cf. recensement de 1993), à savoir:

- le fang: 258601 locuteurs au Gabon

- le yipunu-gisir: 251954

- le yinzebi-duma: 113656 
- l'omyènè: 48 767. Cependant cette langue est en faveur près des élèves de Libreville (motif: communiquer avec ses camarades)

- le mbede-teke: 82 890. Devant la disparition annoncée du mbédé, la Fondation a opté pour le lembaama

- l'ikota-kele: 71351

- l'okandé-tsogo: 32799.

- Et depuis 2000, le vili et le gisir.

Nous avons placé ci-dessous l'omyènè en $2^{\mathrm{e}}$ position car c'est l'une des langues très demandée.

Dans ce tableau, l'on peut observer le niveau de fréquentation des cours de langues au Collège Quaben. Et c'est là un reflet typique des pourcentages retrouvés dans les autres établissements, pour l'année scolaire 2000-2001, avec un total de 500 élèves de la 6ème à la 3ème.

\begin{tabular}{|c|c|c|c|c|c|c|}
\hline Langues & $6^{\text {ème }}$ & $5^{\text {ème }}$ & $4^{\text {ème }}$ & $3^{\text {ème }}$ & Total & Pourcentage \\
\hline Fang & 48 & 50 & 39 & 46 & 182 & $36,40 \%$ \\
\hline Omyènè & 30 & 30 & 37 & 39 & 136 & $27,20 \%$ \\
\hline Yірипи & 22 & 26 & 25 & 25 & 98 & $19,60 \%$ \\
\hline Yinzebi & 17 & 10 & 15 & 17 & 59 & $11,80 \%$ \\
\hline Lembaama & 6 & 6 & 6 & 7 & 25 & $05,00 \%$ \\
\hline
\end{tabular}

Tableau 1: Fréquentation au cours de langues dans le Collège Quaben (20002001)

Comme on le remarque, la langue fang est la plus demandée et nous savons que les enfants partent chez les grands-parents pendant les grandes vacances et apprennent la langue. En deuxième position, la langue omyènè, souvent étudiée par amitié pour un ami ou une amie, par les locuteurs fang ou yipunu. Les langues ghetsöghö et ikota sont peu demandées ; par contre il y aurait des apprenants en gisir et vili, langues qui sont proposées dans le nouveau manuel.

Pour des raisons pratiques, les élèves des classes de 4 ème et 3ème, peu nombreux, sont parfois (et selon les établissements) regroupées par langue, par économie d'enseignants.

Le BEPC blanc organisé dans quelques lycées privés en avril 2001 a présenté les résultats ci-après pour le Collège Quaben et l'Institution Immaculée Conception. 


\begin{tabular}{|l|r|r|r|}
\hline Etablissements & Nombre d'admis & Effectifs Présentés & Pourcentages \\
\hline Quaben & 76 & 118 & $\mathbf{6 4 , 4 0 \%}$ \\
\hline Immaculée & 79 & 122 & $\mathbf{6 4 , 7 5 \%}$ \\
\hline
\end{tabular}

Tableau 2: Résultats du BEPC blanc pour le Collège Quaben et l'Institution Immaculée Conception

Ces résultats au-dessus de la moyenne montrent à quel point les élèves eux-mêmes font l'effort d'apprendre les langues locales.

D'autre part, des soirées culturelles dénommées Soirées des langues gabonaises ont été souvent organisées avec succès et beaucoup d'engouement en fin d'année scolaire par les établissements qui expérimentent la méthode Rapidolangue.

Revalorisant les langues nationales, ces soirées ont permis de se rendre compte de l'intérêt porté par les élèves et les parents d'élèves à l'enseignement des langues gabonaises. Elles sont une réussite de par la richesse de leur contenu et la présence effective des parents. Elles permettent par ailleurs de présenter des sketches en langues (tels qu'un journal télévisé ou des scènes de marché) et des danses traditionnelles, ce qui prouve à souhait que certaines de nos danses sont encore vivantes.

Enfin, ajoutons que la COLANG-EST (Commission des Langues de l'EstCameroun) et le CERLAC (Centre d'Etude et de Recherche sur les Langues d'Afrique Centrale) de l'Université de Czestochowa ont commencé l'introduction de la Collection RAPIDO dans l'est du Cameroun. Quatorze langues locales, parfois très éloignées les unes des autres, ont été traduites pour les classes maternelles et celles de la SIL (Section d'Initiation au Langage) équivalentes au CP1 (Cours préparatoire) : maka, baka, gbaya, ewondo, basaa, bebil, kako, fe'efe'e, bamvele, mbimo, pol, yangele, mezime, medumba. Seules, cinq ont été retenues dans des classes d'établissements privés de Bertoua comme étant les plus parlées. Il s'agit du gbaya, du maka, de l'ewondo (le fang du Gabon), du kako et du fe'efe'e (langue de l'ouest). Autorisé à titre expérimental par le Ministère de l'Education Nationale, cet enseignement se poursuit en dépit de difficultés financières d'édition. Initié également en 6e-5e en 2003-2004, il a été interrompu pour les raisons organisationnelles internes et non par contestation de la méthode qui a déjà fait ses preuves. L'expérience camerounaise est très instructive dans ce sens que des enseignants formés à l'écriture scientifique par la SIL (ne pas confondre, ici : la Société Internationale de Linguistique) et la maîtrisant, ont préféré adopter la méthode d'écriture prônée par RAPIDOLANGUE pour les motifs suivants :

- la facilité d'écriture

- la joie de pouvoir parler dans sa langue 
- le plaisir d'écrire dans sa langue sans avoir fait d'études spéciales en linguistique

- l'enthousiasme des élèves et des enfants

- le dynamisme des enseignantes en maternelles tentées par les comptines en langues, alors qu'il leur était interdit jusqu'ici de le faire.

- La facilité de lecture des textes réécrits selon l'écriture proposée par RAPIDOLANGUE, même pour des personnes ne connaissant que l'alphabet latin. Il est évident que les tons n'ont pas été écrits dans les manuels, mais les enseignants ont été formés pour leur application restreinte.

A l'inverse, certains enseignants se sont détournés de l'écriture et de l'enseignement en alphabet scientifique en raison de :

- la fatigue intellectuelle provoquée par la lecture continue en alphabet scientifique,

- le manque de suivi par le Ministère de l'Education Nationale

- le désintérêt des langues provoqué par les difficultés de lecture. Certains en sont revenus à traduire directement les textes à partir de la langue française alors qu'ils disposaient des mêmes textes en alphabet scientifique.

Pour conclure ce passage, disons que la méthode RAPIDOLANGUE a aussi été traduite dans les langues suivantes mais sans être exploitée: sango (RCA), kinyarwanda (Rwanda), lingala-munukutuba/kikongo-laari (Congo et RDC).

\section{LA QUESTION DU SYSTEME D'ECRITURE}

\section{SONS ET ORTHOGRAPHE SELON LA METHODE RAPIDOLANGUE}

\section{LES VOYELLES :}

- a se prononce comme dans "pas"

- ä ou â est une voyelle prolongée fréquente dans les langues bantu. Elle s'écrit aa en Afrique de l'Ouest comme dans "maam", grands-parents en wolof; lembaama au Gabon

- e se prononce comme dans "été" sauf en langue fang où le "e" est muet comme dans le français "me". Il est écrit "ë" dans les autres langues lorsqu'il est muet.

- è se prononce comme dans "très"

- i se prononce comme dans "si"

- o se prononce comme dans "dos"

- ö se prononce comme dans "note"

- $\mathbf{u}$ se prononce comme dans "vous"

- $\quad$ ü se prononce comme dans "tube". 


\section{LES SEMI-VOYELLES:}

- y se prononce comme dans "pied" ou l'anglais "yes"

- $\quad$ w se prononce comme dans "oui" ou l'anglais "we".

\section{LES CONSONNES}

Les consonnes $\mathbf{b}, \mathbf{d}, \mathbf{f}, \mathbf{g}, \mathbf{k}, \mathbf{l}, \mathbf{m}, \mathbf{n}, \mathbf{s}, \mathbf{t}, \mathbf{z}$, se prononcent à peu près comme en français. Notez que:

- g est toujours dur comme dans "gare" ou "gant".

- s se prononce comme dans "si", jamais comme z. Il ne se double pas.

- $\quad \mathbf{z}$ représente un son intermédiaire entre le z de "zéro" et le j dans "jeu", dans la plupart des cas.

\section{LES CONSONNES COMBINEES}

Leur prononciation est à apprendre par l'usage. Signalons cependant quelques cas.

- ng son nasalisé qui se rapproche de "ing" dans singing" en anglais

- gh ou h se rapproche de la jota espagnole ou du ch allemand

- ny se rapproche du son gn dans "agneau"

- $\quad \mathbf{d y}$ ou dj se rapproche du son "dion", mais varie suivant la langue. Quelquefois, il se rapproche de $\mathbf{d z}$.

Remarque : Les règles d'écriture sont extraites, pour la plupart, de l'écriture proposée dans "Idiomes Gabonais" de Raponda-Walker (1998a), écriture qui a guidée les premiers pas des missionnaires et des chercheurs dans la stabilisation des langues en Afrique, à la fin du siècle dernier. Très proches de l'alphabet phonétique, elles font appel aux principes énoncés par Charles Sacleux (1905) mais une recherche d'uniformisation a été faite pour permettre l'apprentissage des langues sans difficultés supplémentaires.

Quant à la prononciation, on se réfèrera en permanence au professeur ou à la cassette : l'usage fait loi. Il appartient donc au professeur d'enrichir ce livre en y ajoutant le vocabulaire local qu'il enseignera à ses élèves et en acceptant toutes les traductions en langues locales. Cette méthode ne permet pas l'écriture des tons puisqu'elle conserve l'écriture des accents ordinaires en langue française. Il est sans conteste que la méthode de RAPIDOLANGUE obéit aux instructions officielles (mentionnées plus haut) des Ministères de l'Education Nationale pour les langues vivantes. Sons et orthographe selon la commission d'orthographe des langues gabonaises (8-10 avril 1999). En admettant, le soulignement, cette méthode permet l'écriture des tons: 


\section{LES VOYELLES}

- a se prononce comme dans "pas". aa : son prolongé. La méthode marque la longueur vocalique par le redoublement de la voyelle (proposition du CLAD, 1962) comme dans "maam", grands-parents en wolof, okaasi, femme en lembaama

- e se prononce comme dans "été". ee : son prolongé du "é". API [ e ]

- $\quad$ e se prononce comme dans "très". API [ $\varepsilon$ ]

- ë se prononce comme dans les mots français «je, te, me, le, demain ». C'est le fameux schwa ou le « e » muet français. API [ ə ]

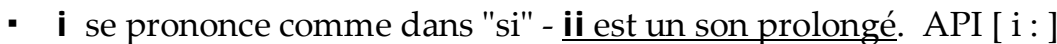

- o se prononce comme dans "dos" - oo est un son prolongé. API [ o : ]

- o se prononce comme dans "note" («o » ouvert)

- $\quad \mathbf{u}$ se prononce comme dans "vous" - uu est un son prolongé. API [ u : ]

- $\quad \underline{\mathbf{u}}$ se prononce comme dans "tube". API [ y ]

LA NASALITE ET LES SEMI-VOYELLES :

- $\quad \underline{\mathbf{n}}$ associé à des voyelles représente les sons nasalisés correspondant à « on-anin » en français comme dans « bon », « enfant » ou « demain ».

- $\quad$ w se prononce comme dans "oui" ou l'anglais "we".

- y se prononce comme dans "pied" ou l'anglais "yes"

\section{LES CONSONNES}

Les consonnes $\mathbf{b}, \mathbf{d}, \mathbf{f}, \mathbf{g}, \mathbf{k}, \mathbf{l}, \mathbf{m}, \mathbf{n}, \mathbf{s}, \mathbf{t}, \mathbf{z}$, se prononcent à peu près comme en français. Notez que:

- g est toujours dur comme dans "gare" ou "gant".

- h son expiré comme dans « ehanda » pour dire la « tombe » en ikota.

- j comme dans « jeu ». Souvent associé avec les digrammes dj ou dz.

- $\mathbf{m b}, \mathbf{m p}$, nd, ng gardent leur valeur de consonnes et s'articulent nettement.

- r est généralement « roulé » dans la plupart des langues bantu

- s se prononce comme dans "si", jamais comme z. Il ne se double pas.

- $\mathbf{z}$ représente un son intermédiaire entre le z de "zéro" et le j de "jeu", dans la plupart des cas.

\section{LES CONSONNES COMBINEES}

Leur prononciation s'apprend également par l'usage. Signalons cependant quelques cas. 
- $\mathbf{d y}$ et $\mathbf{d j}$ se rapprochent du son "dion", mais varient suivant la langue. Quelquefois, ils se rapprochent de dz.

- gh se rapproche de la jota espagnole ou du ch allemand « ach »

- $\quad$ g (généralement en fin de mot) son nasalisé qui se rapproche de "ing" dans «singing » en anglais. Exemple : « lempangu = la langue fang en lembaama). API [n]

- ny ou gn se rapproche du son gn dans "agneau". Exemple : «nyama », la « viande » en yipunu (prononcer gnama). API [ nj ]

\section{LES TONS}

Les langues bantu sont des langues à tons. De nombreux pays ont abandonné la notation des tons car elle provoque une surcharge. Néanmoins, afin d'éviter des confusions regrettables, il est indispensable de les indiquer au Gabon par des signes appropriés afin d'éviter des homonymies. Les tons seront donc marqués de la manière suivante chaque fois que nécessaire:

- les tons bas (par un accent grave). Ex. : è

- les tons hauts (par un accent aigu). Ex. : é

- les tons moyens (par un surlignement) : $\overline{\mathrm{e}}$

\section{AMELIORATIONS DE L'ORTHOGRAPHE DANS RAPIDOLANGUE}

Finalement, l'élaboration du manuel RAPIDOLANGUE a tenu compte des principes ci-dessus. Ont été adoptés en Assemblée générale:

1. les propositions de la commission des 8-10 avril 1999 sur l'orthographe des langues (Dodo-Bounguendza 1998/1999, 2001)

2. les principes de simplicité et fonctionnalité ainsi que les signes diacritiques proposés par le LUTO-DC (Laboratoire Universitaire de la Tradition Orale et des Dynamiques Contemporaines) et la commission ad hoc susmentionnée, à savoir le soulignement, afin de préserver l'écriture des tons si nécessaire (ex : $\underline{\mathrm{e}}$ $=[\varepsilon]$ au lieu de /è/ qui porte une autre signification)

3. l'adoption des tons principaux indispensables. Si les nouveaux manuels n'ont pas encore inscrit les tons sur certains homonymes ou autres, c'est faute de volontaires pour le faire. Mais le principe a été adopté : il privilégie les tons hauts (accent aigu) indispensables à la compréhension. Les tons bas (accent grave) ou moyens pourront être mentionnés pour la clarté de la compréhension. Il est préférable que l'enseignant les écrive au tableau noir tout comme l'on utilise l'API dans l'enseignement de l'anglais.

4. La longueur vocalique par le redoublement de la voyelle (ex. lembaama)

5. L'adoption du gh commun à toutes les langues gabonaises pour le son contesté du [x] (ex. [xalam] : nom d'une danse célèbre au Sénégal). Nous écrirons aussi 
« mvöghe » en langue fang; " ghetsöghö », etc. Pour les mêmes raisons, nous évitons l'écriture $\mathbf{d u} \mathbf{j}\left[\mathrm{d}_{3}\right]$, toujours mal prononcé comme dans Banjul [bãdzul], la capitale de Gambie et le c [t $\left.\int\right]$ qui prête à confusion, et nous préférons le ny pour 'nyama' au lieu de 'gnama'.

\section{CONCLUSION}

Il est vrai qu'il n'est pas suffisant de parler une langue pour être capable de l'enseigner, il faut aussi appliquer la méthodologie spécifique à l'enseignement des langues vivantes pour être compétent. C'est le rôle des modules éducatifs des années 2000-2002 à 1'Ecole Normale Supérieure. Dès le début, les missionnaires avaient introduit quelques langues locales dans leurs établissements mais avaient dû abandonner leur enseignement sous la pression des autorités coloniales. Ne pouvons-nous pas dire aujourd'hui, après Gassama, que les langues négroafricaines, langues à tradition orale, doivent savoir se servir des techniques des langues écrites pour se maintenir ? N'est-il pas temps de réintroduire nos langues dans le système éducatif ? Des pays d'Afrique Centrale le font déjà, pourquoi pas le Gabon? Les expériences entreprises dans les lycées catholiques et les lycées d'Etat méritent toute notre attention même si pour des raisons financières l'expérience risque de tourner court, un manuel d'apprentissage a le mérite d'exister, des grammaires ont été éditées, il ne reste plus qu'à franchir «le Rubicon » et introduire nos langues dans l'enseignement avec le soutien des Autorités compétentes.

Quelles langues enseigner? La question n'est pas importante car dans l'immédiat, toutes les langues peuvent l'être si l'on s'en tient aux plus parlées. Une langue nationale verra-t-elle le jour au Gabon ? Les efforts des linguistes et principalement du Ministère de l'Education Nationale aboutiront-ils? L'avenir nous le dira, la décision est entre les mains des décideurs, mais il est primordial qu'une décision politique intervienne rapidement sous peine de rendre inutiles tous les efforts entrepris pour la sauvegarde de nos langues. Les difficultés rencontrées ne semblent pas venir de la méthode qui a prouvé son efficacité mais plutôt de son application par certains enseignants qui ne respectent pas ses principes et utilisent un vocabulaire supérieur au niveau demandé. Le bilan a été fait régulièrement par les enseignants eux-mêmes chaque fin d'année scolaire.

RAPIDOLANGUE a donné naissance à des petits frères en gestation:

1. RAPIDOMATER pour les écoles maternelles

2. RAPIDOPREPA, pour les cours préparatoires

3. RAPIDELEM, pour les cours élémentaires

4. RAPIDOMOYEN, pour les cours moyens. 
Appel à contribution est lancé ce jour, comme en 1995, à toutes les bonnes volontés, sachant que la Fondation Raponda-Walker est une association créée en 1993 sur la base du bénévolat. S'il est vrai que l'expérience a démarré dans l'enseignement catholique - grâce à ses statuts particuliers -, il n'en demeure pas moins vrai que le Gouvernement gabonais a été informé dès le départ et a autorisé cet enseignement à titre expérimental et qu'une dizaine de traducteurs appartiennent à l'enseignement secondaire ou supérieur. Nous connaissons la suite. Rappelons que la Fondation est prête à jouer le jeu d'une franche collaboration mais pas à n'importe quelle condition. Elle ne sacrifiera pas ses principes originels à n'importe quel prix. Je renvoie donc à l'ouvrage de Daniel Franck Idiata (2002) qui aborde nombre de problèmes en suspens. Ne craignons pas la confusion qui peut se créer avec l'apprentissage des langues internationales, car il importe avant tout de sauvegarder nos langues. La survie de notre culture en dépend.

\section{REFERENCES}

Calvet, L-J. 1987. La Guerre des Langues. Paris: Payot.

Centre de Linguistique Appliquée de Dakar. 1962. Ecriture des langues nationales du Sénégal. C.L.A.D.

Collection IHEF 2000a. Plurilinguisme et diversité culturelle. Institut des Hautes Etudes Francophones. Cassette vidéo.

Collection IHEF 2000b. Le génie linguistique, Université Audiovisuelle Francophone. Cassette vidéo.

Cours de lingala, 1986. L'Epiphanie. Kinshasa.

Crystal, D. 2000. S.O.S. Langues en péril. Courrier International n ${ }^{\circ} 486 \mathrm{du} 1^{\mathrm{er}}$ mars 2000.

Département de Linguistique, 1979. Propositions pour l'orthographe des langues congolaises, 16-24 novembre 1979. Brazzaville: FLSH/Université Marien Ngouabi.

Dodo-Bounguendza, E. 2001. Réflexions sur l'enseignement des langues gabonaises dans le système éducatif. Documents dactylographiés.

Dodo-Bounguendza, E. 1998/1999. Rapport d'activités: promotion et intégration des langues nationales dans le système éducatif. Libreville: Ministère de l'Education Nationale.

Hubert, J. 2001a. «Faut-il enseigner les langues gabonaises ? ». Conférence du $1^{\mathrm{er}}$ juillet 2001. Port-Gentil.

Hubert, J. 2001b «La méthode Rapidolangue : Bilan des 5 années d'application ». Conférence du 1er juillet 2001. Port-Gentil.

Hubert, J. 2002. «La méthode Rapidolangue». GRELACO-SIL : Atelier sur les langues gabonaises à l'école. 29 mars 2002. Libreville. 
Hubert, J. 2003. «Rapidomater 2 », Ed. Raponda-Walker/Colang-Est (14 langues au Cameroun, 1 langue au Gabon)

Hubert, J. 2003. « Rapidosil », Ed. Raponda-Walker/Colang-Est (5 langues)

Ibrahim, I. 2000. La situation linguistique du Gabon examinée avant la clôture du Colloque international sur les villes plurilingues. L'UNION du 28 septembre 2000.

Idiata, D. F. «Il était une fois les langues gabonaises » 2002, Ed. Raponda-Walker

Idiata, D. F. « Le pari des langues » 2002, Ed. Raponda-Walker

Kere-Ouedraogo, A. 1993. Lexique Mooré-Français, Français-Mooré, ACCT

Kwenzi-Mikala, J.T. 1987. "Contribution à l'inventaire des parlers bantu du Gabon ». Pholia 2 : 103-110. Lyon: Université Lumière Lyon 2.

Kwenzi-Mikala, J.T. 1990. "Quel avenir pour les langues gabonaises?». Revue Gabonaise des Sciences de l'Homme 2 : 121-124. Libreville: LUTO/Université Omar Bongo.

Kwenzi-Mikala, J.T. 1998. «Parlers du Gabon: classification du 11-12-97». Raponda-Walker, Les langues du Gabon. Libreville: Editions RapondaWalker.

LUTO, 1990. Revue gabonaise des Sciences de l'Homme $\mathrm{n}^{\circ} 2$. Actes du séminaire des experts sur l'Alphabet Scientifique des langues du Gabon. LUTO/Publications de l’Université Omar Bongo.

Medjo-Mve, P. 2001. Le haoussa : langue gabonaise ? L'UNION du 22 mars 2001.

Minisiteri Y'amashuri Abanza N'ayisumbuye 1988. Ikinyarwanda, Umwaka wa Munani. Gashyantare.

Minisiteri Y'amashuri Abanza N'ayisumbuye Ikinyarwanda 1986. Umwaka wa Karindwi. Gashyantare

Minisiteri Y'amashuri Abanza N'ayisumbuye. 1983. Urutonde rw'Amuga (Dictionnaire Kinyarwanda-Français). Kamena.

Nadaillac, L. 1992a. Lexique Inzebi-Français. Université Lumière Lyon 2.

Nadaillac, L. 1992b. Lexique Isangu-Français. Université Lumière Lyon 2.

Overduive-Jacob, 1998. Twige inkinyarwanda, Manuel d'apprentissage de la langue rwandaise. Palloti-Presse, 3e édition.

Programme de l'Union Européenne. 1980. Grammaire de la communication et vocabulaire lexical.

Raponda-Walker, A. 1998a. Les langues du Gabon. Libreville: Editions RapondaWalker.

Raponda-Walker, A. 1998b. Eléments de grammaire (Fang, Gisira, Ghetsöghö, Ebongwè). Libreville: Editions Raponda-Walker

Raponda-Walker, A. 1934/1995. Dictionnaire Mpongwè-Français. Metz/St-Paul Versailles

Raponda-Walker, A. 1961/1995. Dictionnaire Français-Mpongwè. Saint-Paul Versailles

Raponda-Walker, A. 1993. 3.000 Proverbes. Libreville: Editions Raponda Walker. 
Raponda-Walker, A. Dictionnaire Tsogo-Français, inédit.

Raponda-Walker, A. Dictionnaire Français-Tsogo, inédit.

Raponda-Walker, A. Lexique Français-Eshira, inédit.

Sacleux, C. 1909. Grammaire des Dialectes Swahilis. Paris: Procure.

Sacleux, C. 1905. Essai de Phonétique. Paris: .Procure.

Sacleux, C. 1903. Introduction à l'étude des langues bantoues. Paris: Institut de laryngologie et orthophonie.

Wiesemann, Sadembouo et Tadadjeu, 1983-1988-2000. Guide pour le développement des systèmes d'écriture des langues africaines. Yaoundé: Coll. Propelca.

Shell et Wiesemann. 2000. Guide pour l'alphabétisation en langues africaines. Yaoundé: Coll. Propelca.

Van Everbroeck 1985. Dictionnaire Lingala-Français, Français-Lingala. Kinshasa: Epiphanie. 


\title{
ALPHABET ET ECRITURE: APPROCHE HISTORIQUE ET CAS DES LANGUES GABONAISES
}

\author{
Hugues STEVE NDINGA-KOUMBA-BINZA \\ CEnTRE For TeXt Technology (CTEXT) \\ NORTH-WESt UNIVERSITY, POTCHEFSTROOM CAMPUS
}

(22602560@nwu.ac.za)

\section{INTRODUCTION}

L'écriture, au sens strict du mot, dérive de la parole, et est définie par Barber (1982: 37) comme une «imparfaite représentation de la parole». La manifestation pratique de l'écriture au sens moderne du terme est marquée par les alphabets et orthographes. Le présent chapitre concerne spécialement l'origine et le développement des alphabets orthographiques ${ }^{1}$. Il s'agit principalement d'une approche historique de quelques processus de confection et/ou d'établissement des alphabets et orthographes pour les langues à écriture phonographiques. En partant d'un bref aperçu des origines mêmes de l'écriture, je fais l'analyse des alphabets existants dans les langues gabonaises après avoir fait état du problème de manière générale dans les langues africaines.

\section{ORIGINES ET DEVELOPPEMENT DES ALPHABETS}

L'histoire de l'alphabet et de l'orthographe remonte à l'invention même de l'écriture. L'écriture apparaît elle-même pour la première fois en forme de pictographe, puis de type cunéiforme avant d'adopter la forme phonographique de nos jours.

\section{DES PICTOGRAPHES AUX CUNEIFORMES}

La communication écrite apparaît dès l'homme paléolithique avec des gravures et peintures rupestres dans des grottes, des images éraflées sur des os ou ivoire, et

1 Voir Thierry Afane Otsaga, dans ce volume, pour la distinction entre les concepts d'alphabet et d'orthographe. 
même des figurines féminines faites d'argile ou de pierre molle. En effet, les écritures égyptienne et akkadienne ${ }^{2}$ avaient été en usage bien avant 3000 ans avant JC et étaient pictographiques à l'origine, les signes orthographiques étant tout simplement des images d'objets.

Barber (1982: 37) considère que ces systèmes pictographiques ne sont pas de l'écriture, au sens moderne du terme, parce que les symboles correspondent non pas à des mots particuliers ou autres items linguistiques mais à des images dont la codification du sens peut être déchiffrable dans n'importe quelle langue. C'était plus ou moins une écriture idéographique à l'image des scripts traditionnels d'orient (mandarin, coréen, nippon, etc.). Mais ces images ont été importantes pour le développement de l'écriture. Car il est largement attesté que c'est bien à partir d'elles que l'écriture dite phonographique s'est constituée avec l'apparition des alphabets.

Le développement de ces formes iconiques en écriture a connu deux principaux processus (Barber 1982 : 39):

- les images furent simplifiées et conventionnalisées de telle sorte qu'elles n'étaient plus reconnaissables comme images ;

- puis, ces images conventionnalisées furent amenées à représenter directement des items linguistiques ( $\mathrm{d}^{\prime}$ abord des mots, puis de syllabes et finalement des sons ou phonèmes) et furent classées dans le même ordre comme unités linguistiques.

Le processus de simplification et de conventionnalisation était lié à la superficie et à l'importance des matériaux utilisés pour écrire. Si certains symboles étaient gravés sur des tablettes d'argile, ou gravés sur du bois ou de la pierre, il y eut une tendance à éviter des courbes et l'écriture devint angulaire. C'est ce processus qui est observable dans une très célèbre et première forme d'écriture (peut-être même le plus ancien véritable système d'écriture, selon Barber 1982: 40) qui fut développée par les Sumériens du Sud de la Mésopotamie entre 4000 et 3000 ans avant JC.

Il est en effet attesté que les scribes sumériens écrivaient sur des tablettes d'argile avec un stylus fabriqué à partir d'un roseau. Au préalable, ils dessinaient de petites images en tirant la pointe du stylus sur l'argile, mais cela n'était vraiment pas efficace, puisque l'argile s'entassait devant le stylus et brouillait l'image.

Plus tard, ils durent simplement appuyer la tête du stylus dans l'argile comme un tampon, produisant ainsi une petite marque en forme de coin à la longueur du tiers d'un pouce ${ }^{3}$. Chaque image était faite $d^{\prime} u n$ ensemble de types de coins 
typiques. Ainsi est née ce qui est appelée l'écriture cunéiforme : le terme cunéiforme signifiant «en forme de coin». La figure ci-dessous (Barber 1982: 40) montre le développement de cinq signes pictographiques sumériens en symboles cunéiformes. Des sept colonnes de cette figure, seules la première et les deux dernières méritent un commentaire. La première colonne présente les mots anglais (respectivement bouf, charrue, verger, soleil et grain), tandis que les deux dernières colonnes sont respectivement des formes classiques du babylonien et de l'assyrien.

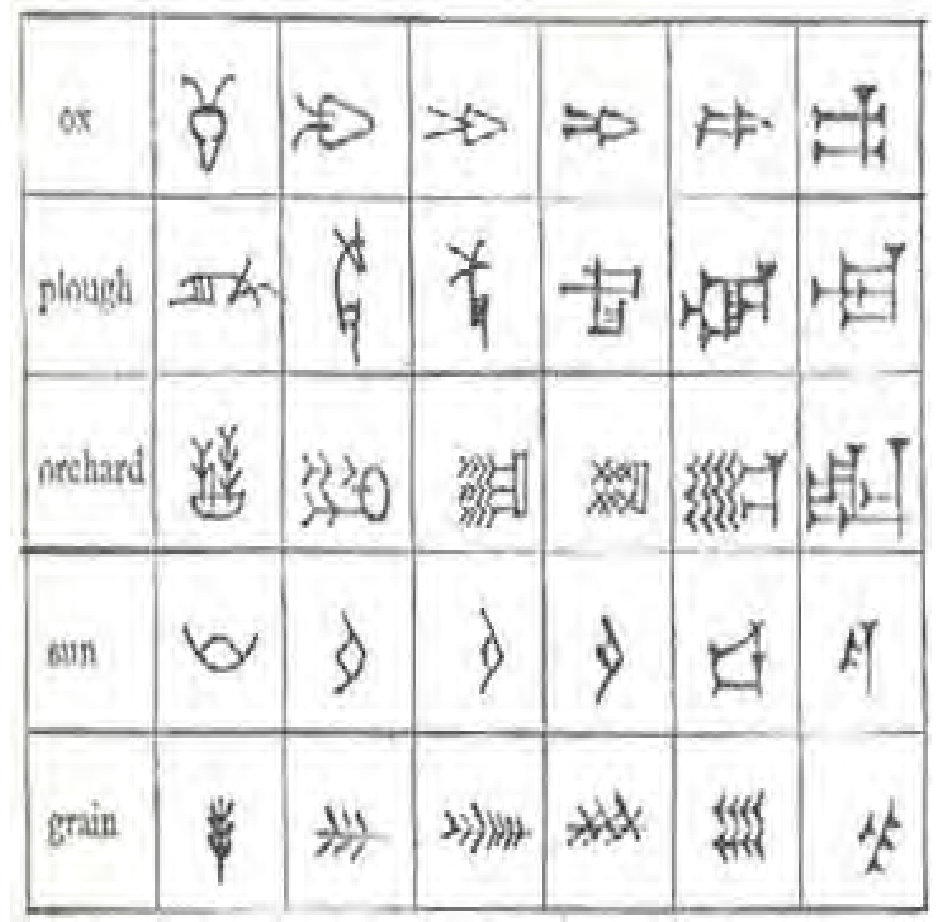

Figure 1: Développement du sumérien en cunéiforme

Par ailleurs, lorsque les scribes écrivaient à l'aide d'un stylo ou un pinceau sur du cuir ou des papyrus ${ }^{4}$, il s'effectuait un développement différent : celui des courbes, qui loin d'être difficile à reproduire, offraient des formes plus simples et plus rapides. C'est de cette manière que l'écriture cursive va se développer. Pour ce genre de développement, Barber (1982: 41) cite le cas du système d'écriture de l'ancien égyptien qui s'est aussi développée à partir des formes pictographiques, les hiéroglyphes (du grec saintes lettres sculptées). Cette dénomination est due au fait que les Grecs pensaient que les Egyptiens utilisaient leur écriture essentiellement pour des inscriptions religieuses. En effet, comme l'indique Barber (op.cit.), l'écriture égyptienne pouvait être aussi bien peinte que sculptée, et était aussi

4 Le papyrus était un tissu, semblable à du papier pour écrire, fabriqué à partir des joncs. 
utilisée pour des documents écrits sur des papyrus. Pour écrire, les scribes faisaient usage d'une sorte de pinceau.

L'écriture hiéroglyphique était utilisée durant toute la période de l'Egypte antique à des fins religieuses, mais pour l'usage quotidien une forme d'écriture un peu plus conventionnalisée, moins compliquée et beaucoup plus rapide à écrire, se développa très tôt à partir même de ces hiéroglyphes. Il s'agit de l'écriture hiératique, c'est-à-dire l'écriture sacerdotale (Barber 1982 : 42). A partir de celle-ci se développa enfin vers 700 ans avant notre ère une forme dite démotique ou l'écriture populaire. La figure ci-après tirée de Barber (1982: 42) présente deux symboles hiéroglyphiques et leur développement en symboles hiératiques et démotiques pour l'écriture de la langue égyptienne.

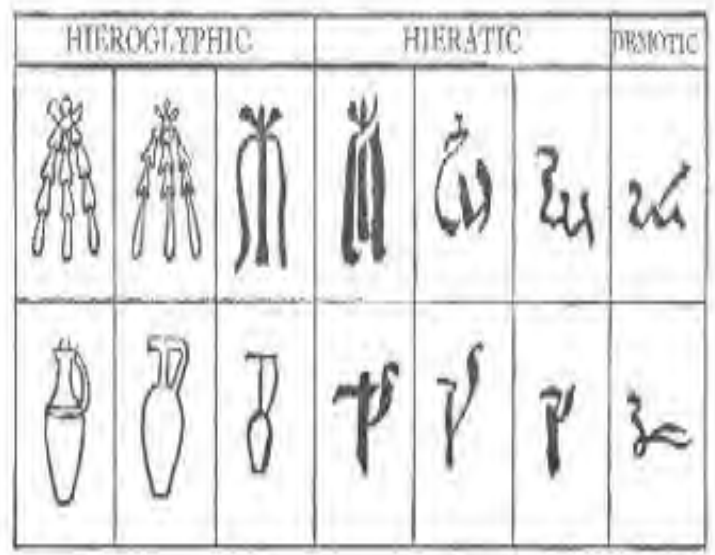

Figure 2: Développement de l'écriture égyptienne

La conventionnalisation de l'écriture comme précédemment vue chez les Sumériens et chez les Egyptiens a connu un développement parallèle ailleurs. C'est le cas non seulement de l'écriture traditionnelle chinoise, mais également celle des scripts phéniciens dont la forme cunéiforme donne son origine à l'alphabet grec (voir Afane Otsaga, dans ce volume, pour des informations plus détaillées sur l'alphabet grec).

\section{DES CUNEIFORMES AUX ALPHABETS}

Le second processus du développement des pictographes en écriture fut le passage de l'orthographe cunéiforme à la représentation directe des items linguistiques par ces images conventionnalisées. Comme mentionné plus haut, ce fut d'abord la représentation des mots, puis des syllabes et en fin de compte des sons ou phonèmes. Ce qui se distinguait nettement de l'orthographe sumérienne qui, en 
réalité, était juste des listes d'objets avec des symboles pour nombre d'entre elles. Barber (1982 : 43) évoque l'exemple selon lequel quatre demi-cercles et une image de tête de bœuf formaient un ensemble qui devrait être lu «quatre bœufs ».

Les premiers alphabets issus du développement de l'écriture cunéiforme proviennent du Proche Orient (Healy 1994). La plupart des chercheurs font remonter l'apparition de l'écriture alphabétique au deuxième millenium avant JC. D'après Healy (1994 : 74), ce développement fut une évolution significative à partir des formes d'écriture qui déjà existaient, telles que le système cunéiforme suméroakkadien et le système hiéroglyphique égyptien.

Les alphabets issus de ces formes se développèrent en systèmes représentant des combinaisons phoniques syllabiques ou des structures phoniques consonantiques. En effet, le développement vraiment crucial de l'écriture fut la phonétisation de celle-ci, c'est-à-dire l'association d'un symbole à un son particulier ou à un groupe de sons.

Par exemple, l'écriture syllabique de l'akkadien qui continuait à user des pictographes stylisés était un système de centaines de signes de syllabes distinctes qui pouvaient être regroupées pour l'écriture d'un mot. Dans ce système chaque signe cunéiforme représentait une syllabe, tels que $b a, l u$ ou ir. Ceci fut une importante innovation dans des systèmes qui étaient habitués à l'usage des signes pour représenter des mots entiers; innovation aussi, parce que le nombre des symboles utilisés se réduisait à environ une centaine.

L'écriture de l'ancien égyptien se développa aussi en système syllabique, et fut particulièrement importante pour le développement $d$ 'une véritable écriture alphabétique, c'est-à-dire une écriture ayant des symboles représentant des phonèmes.

Cependant, il faut préciser que depuis Gelb (1963) ces alphabets originels sont considérés non pas comme des alphabets en tant que tel, mais plutôt comme des types de syllabaires dans lesquels chaque signe constituait une consonne qui pouvait être suivie de n'importe quelle voyelle. D'où le terme d'alphabet consonantique qui leur est attribué.

Dans le système égyptien par exemple, les voyelles n'étaient pas indiquées. La plupart des signes (environ 80) représentaient chacun un groupe de deux consonnes (Barber 1982: 45). Par exemple, le symbole pour une maison (par) représentait le groupe $p r$, et cela pouvait signifier par, per, apr, epr, epra et ainsi de suite. Il y avait de même 24 signes représentant chacun seulement une consonne qui pouvait être précédée ou suivie de n'importe quelle voyelle. Le symbole représentant la bouche $(\mathrm{ra})$ par exemple était la consonne $r$, et pouvait signifier $r a$, ar, re, er, etc. 
Healy (1994 : 74) justifie le point de vue de Gelb par le fait que l'inventeur ou les inventeurs du premier alphabet ont tout simplement isolé les sons consonantiques et $n^{\prime}$ ont accordé aucune attention aux voyelles. Toutefois, il est supposé que la nonreprésentation de voyelles dans les alphabets utilisés pour l'écriture des langues sémitiques est le reflet de la distinction des rôles entre voyelles et consonnes dans lesdites langues.

Lorsque les peuples sémitiques occidentaux vivant autour du littoral oriental de la Méditerranée développèrent une écriture, ils le firent en s'inspirant des Egyptiens, justement à partir des fameux 24 signes consonantiques évoqués plus haut.

Il eut par la suite quelques tentatives dans ces langues sémitiques pour représenter les voyelles, puisque dans des premiers scripts araméens et ensuite dans ceux du hébreux certains signes consonantiques - h, w et y - finir par être aussi utilisés, dans des conditions restreintes, pour la représentation des voyelles (Healy 1994). Ce sont ces consonnes qui furent appelées lettres vocaliques dans les langues sémitiques.

L'alphabet au sens moderne du terme apparut pour la première fois avec les Grecs qui ayant adopté l'alphabet consonantique des Phéniciens se mirent à faire usage de certains signes non pertinents en langue grecque pour représenter des voyelles (Afane Otsaga donne dans ce volume des informations plus précises sur cet aspect). Allant au-delà de l'alphabet sémitique, les Grecs abordèrent ainsi l'étape de fixation des symboles pour voyelles. Certains sons du phénicien n'existaient pas dans la langue grecque. Les Grecs durent utiliser les symboles de ces sons pour la représentation des voyelles.

La prise en compte des voyelles de ce nouvel alphabet va s'avérer une faiblesse intrinsèque qui plus tard va être rectifiée pour des langues comme l'arabe, le syrien, l'éthiopien et l'hébreu par l'introduction de nouveaux signes

La fixation qui distingue les symboles pour consonnes des symboles pour voyelles fut finalement l'ultime phase de la conception et de l'établissement d'un système d'écriture où les symboles renvoient aux phonèmes de la langue.

Bien plus tard, des systèmes alphabétiques - tel que l'alphabet latin utilisé dans la plupart des langues européennes- dérivèrent de l'accomplissement de l'orthographe grecque dont le grand avantage fut le nombre pas trop important des symboles nécessaires. Ceci rend l'alphabétisation universelle possible (Barber 1982 : 46).

La figure (Barber 1982 : 43) qui suit montre certains symboles de l'alphabet latin $(A$, $R, N$, $S$, et $B$ ) et leur probable développement sur la base des hiéroglyphes de 3000 ans avant JC. La première colonne montre les mots - respectivement bœuf, tête, serpent, montagnes et cour - en anglais. De la deuxième à la dernière nous avons 
respectivement la forme hiéroglyphique, la forme sinaïtique (de Sinaï), la forme ouest-sémitique, la première forme grecque, la forme finale grecque et enfin la forme latine.

\begin{tabular}{|c|c|c|c|c|c|c|}
\hline & 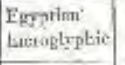 & Sünaí & $\begin{array}{l}\text { West } \\
\text { Sentitic }\end{array}$ & $\begin{array}{l}\text { Fant' } \\
\text { Greek }\end{array}$ & $\begin{array}{l}\text { tats } \\
\text { Gromir }\end{array}$ & Latisa \\
\hline$u x$ & ?. & $d$ & 4 & $\not$ & A & A \\
\hline head & & & 94 & $4 P$ & P & $\mathrm{R}$ \\
\hline snake & $q$ & & $y$ & 4 & $N$ & $N$ \\
\hline $\begin{array}{l}\text { mount- } \\
\text { ains }\end{array}$ & 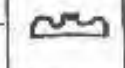 & $\omega$ & w & $\leqslant$ & $\Sigma$ & $S$ \\
\hline $\begin{array}{l}\text { court- } \\
\text { yard }\end{array}$ & J & $\longmapsto$ & 49 & $\Rightarrow 8$ & $B$ & B \\
\hline
\end{tabular}

Figure 3: Développement de cinq lettres à partir des hiéroglyphes égyptiens

Pour conclure cette section, un autre aspect de l'étude du premier alphabet qui nécessite d'être brièvement mentionné ici est le développement et la fixation de l'ordre des lettres. Il y a une évidence nette que même les premiers textes étalent tout simplement les lettres dans un ordre alphabétique. Ces textes, habituellement des exercices ou des manuels scolaires, sont appelés des abécédaires bien que le rangement réel n'était pas similaire à celui des alphabets modernes (Healy 1994).

Dans la section qui va suivre j'aborde les problématiques d'alphabets et orthographes dans les langues modernes à tradition orale.

\section{LES PROBLEMATIQUES DANS LES LANGUES MODERNES A TRADITION ORALE}

La confection d'un alphabet à visée orthographique est le fait de choisir une forme d'écriture pour l'orthographe d'une langue qui n'en avait jamais eue. C'est une façon d'encoder visuellement la langue en vue de son usage efficient dans la communication écrite. De nos jours un grand nombre de langues parlées en Afrique, en Amérique latine, en Inde et dans d'autres parties de l'Asie et dans le Pacifique ont été dotées d'une forme écrite. 
Des techniques linguistiques telle que la phonémique furent développées dans le contexte direct des problèmes posés par la création des formes d'écriture convenables aux langues à tradition orale.

D'après Florian Coulmas (1996: 12), du point de vue purement linguistique un tableau de transcriptions phonologiques qui soit systématiquement transparent, précis et économique, serait le système d'orthographe idéal. Toutefois, ces critères sont connus pour déterminer le succès d'une orthographe nouvellement proposée seulement pour un nombre de langues assez limité.

Car les écritures et les conventions orthographiques ne sont jamais socioculturellement neutres pour leurs utilisateurs. En effet, à défaut d'être seulement des instruments pour une pratique naturelle, ils sont parfois des systèmes symboliques d'une signification sociale importante, puisqu'ils portent souvent en eux-mêmes des connotations culturelles et politiques. C'est le cas dans certains pays de l'Afrique orientale (Kenya, Tanzanie, etc.) qui ont émis des réserves à l'adoption d'un système d'orthographe des langues africaines similaire à l'alphabet anglais du fait de l'hégémonie de l'anglais sur les langues locales.

Il peut y avoir des raisons non linguistiques pour des communautés linguistiques de vouloir une orthographe similaire ou différente par rapport à une autre langue. Dans certains pays tel que l'Inde, l'adoption d'un système d'écriture a été abordée dans le contexte de l'héritage littéraire du pays et dans le contexte des programmes d'alphabétisation du gouvernement. L'alphabétisation en langues vernaculaires est développée comme un pont pour l'alphabétisation dans l'une des langues littéraires régionales. Cette notion implique qu'une nouvelle orthographe pour une langue vernaculaire devra être conçue sur le modèle de la langue dominante de la région où la langue vernaculaire est parlée.

Ce principe semble avoir été adopté par les missionnaires qui dans certains pays comme le Gabon introduisirent dès les premières heures de l'époque coloniale les langues locales non seulement dans le système éducatif général, mais surtout dans l'éducation religieuse. Leurs systèmes d'écriture étaient relativement proches des langues européennes qui servaient de langues de communication large dans les colonies françaises, anglaises, espagnoles ou portugaises respectives. La proximité entre les deux systèmes facilite le transfert du niveau de développement d'un système à l'autre.

Par ailleurs, l'adéquation linguistique et l'économie n'ont aucun prestige culturel. Mais les orthographes existantes, aussi irrégulières et défectueuses qu'elles peuvent paraître, sont le plus souvent prestigieuses du point de vue culturel. Dès lors, l'acceptabilité d'un nouvel alphabet par une communauté linguistique donnée n'est entièrement pas déterminée par sa taille et sa compétence sur le plan linguistique. 
Les différences dialectales constituent également un autre problème qu'il faut considérer dans la confection de nouvelles orthographes. S'il faut avoir une langue écrite uniforme, des choix doivent être faits à l'endroit où différentes variantes présentent une diversité de formes différentes. Cependant, ces choix ne peuvent non plus être déterminés sur la base des seuls critères linguistiques. Car de tels choix peuvent influencer le développement d'une variété standard qui nécessiterait une considération minutieuse. Le plus souvent les locuteurs de différentes variétés d'une même langue tendent à comparer celles-ci entre elles (voir Mavoungou dans ce volume). L'idéal est que la variété qui va être écrite constitue un point commun de référence pour les locuteurs de tous les dialectes.

Enfin, dans la confection d'alphabet il va falloir trouver un juste milieu entre les exigences linguistiques et extralinguistiques. Coulmas (1996: 13) suggère qu'une nouvel alphabet orthographique devra se baser sur une variante de la langue acceptable par la majorité de la communauté linguistique; il devra être phonémique selon l'importance que cet exigence n'entrave pas son acceptabilité et, où c'est utile, il devra aussi inclure des informations lexicales et morphophonologiques; il doit être facile à apprendre et à écrire à la fois à la main et au moyen d'un équipement d'impression disponible (traitement de texte) ; il doit être facile à lire; et il doit transcender le moins possible les limites de l'inventaire des signes de l'orthographe de la langue majeur de contact. Je renvoie dans ce volume à Afane Otsaga pour d'autres détails sur la critériologie de la confection des alphabets et orthographes.

\section{L'ECRITURE DES LANGUES AFRICAINES}

\section{UN APERÇU HISTORIQUE}

Nous venons de voir plus haut que l'écriture est apparue en Mésopotamie vers 3000 ans avant notre ère. L'invention des hiéroglyphes par les Egyptiens vers 2850 avant notre ère est l'un des témoignages que l'écriture n'est pas un fait étranger en Afrique (Touré 1990 : 55). Des nos jours, cet écriture égyptienne se trouve encore gravés sur les parois des temples et tombes ou encore sur des feuilles de papyrus en Egypte et dans les grands musées du monde.

Ahmadou Touré (1990: 56) affirme que l'Afrique subsaharienne a apporté sa contribution pendant l'ère coloniale. "Des témoignages encore vérifiables aujourd'hui existent comme par exemple les poids à peser l'or chez les Ashantis, le tifinagh chez les Touaregs, les peintures rupestres trouvées dans de nombreuses régions, les bas-reliefs des palais d'Abomey, les statuettes royales kuba du Congo, les symboles graphiques de la société Komo chez les Bamanas, etc. » (Touré 1990 : 56). 
Le développement particulier et individuel de ces systèmes d'écriture n'a pu être possible compte tenu, à mon avis, du contact précoce avec le monde occidental au travers des invasions successives arabe et européenne dès le Xème siècle et au travers de la colonisation qui s'en est suivie. La création pendant la colonisation des écoles en langues européennes a favorisé non seulement la langue du colonisateur mais aussi son système d'écriture.

Les missionnaires et les administrateurs coloniaux dans leurs objectifs respectifs de l'évangélisation et de la connaissance des langues locales ont été les premiers à doter les langues africaines de systèmes d'écriture utilisant l'alphabet latin. Les grammaires, les manuels d'apprentissage ou d'enseignement, les dictionnaires et autres lexiques étaient tous écrits en alphabet latin et les symboles représentaient les sons de la langue en fonction de la langue d'origine du rédacteur.

Pour le xhosa par exemple, on peut lire dans la grammaire de McLaren (1936) que le même alphabet est utilisé aussi bien pour écrire l'anglais que pour écrire le xhosa, à l'exception des sons consonantiques [6], [r], et [J]. Ce qui faisait de l'orthographe xhosa de l'époque un système de 29 lettres dont 5 voyelles ( $a, e, i, 0$, u) et 3 clicks (c, q, x). Comme on peut l'observer les clicks (qui jouent le même rôle que les consonnes dans la langue) qui n'existent pas dans les langues européennes ont dû être représentés par les symboles $c$, $q$ et $x$ qui ne peuvent avoir de signification particulière en langues africaines. Ce système d'orthographe du xhosa s'est poursuivi tout au long de la période coloniale et de la période d'apartheid.

A la différence de certaines langues dans bon nombre de pays africains, l'adaptation du système du xhosa (et c'est le cas de la plupart des langues $\mathrm{d}^{\prime}$ Afrique australe) à la langue européenne (l'anglais dans toute l'Afrique australe) eut à tenir compte du système phonologique particulier de la langue. Ce qui ne fut vraiment pas le cas en Afrique de l'ouest et du centre, particulièrement dans les régions anciennement colonisées par la puissance française.

Dans ces régions, les premiers alphabets adaptés à la phonologie des langues apparurent seulement lorsque des missionnaires comme Raponda-Walker commencèrent à s'intéresser à la description de ces langues africaines.

Il faut préciser que la situation des langues africaines dans les colonies belges (la région dite du Congo-Belge) et anglaises semblait bien meilleure que dans les colonies françaises où le développement des langues locales était difficilement une préoccupation. Kwenzi-Mikala (1990 : 121) le précise en ces termes:

«Le statut des langues africaines varie selon les pays. Dans les colonies britanniques de la Gold Coast - l'actuel Ghana - et du Nigeria, et dans la colonie belge du Zaïre, les langues africaines ont pu servir de véhicule de l'enseignement et de matière à enseigner. Cette 
politique se poursuit actuellement. Il n'en fut rien dans les possessions françaises en général...».

De même, ainsi que le dit Touré (1990: 58), «les anciennes colonies anglophones, comme le Nigeria, [...] avaient durant toute la période coloniale transcrit régulièrement et utilisé leurs langues dans les premières années du système scolaire ».

Il faut ajouter que les efforts des missionnaires des pays francophones à vouloir introduire dans l'éducation scolaire des jeunes africains (Mayer 1990) furent estompés par l'ordonnance française de février 1922 qui, à la suite de celle de Villers-Cotterêts en 1539, réglementait l'enseignement privé et l'enseignement religieux dans les territoires français. "L'enseignement général devait être dispensé exclusivement en français et l'enseignement religieux dans les langues locales » (KwenziMikala 1990 : 123).

Il est évident que dès lors qu'une langue est écartée du système éducatif, cela constitue un véritablement frein à son épanouissement en général, et au développement de son système d'orthographe en particulier. Ainsi la forme d'écriture des langues africaines que trouvent les indépendances dans ces colonies françaises résulte essentiellement des traductions de la Bible et des catéchismes, et de l'écriture des chants et cantiques en langues locales.

Dans la plupart des pays, le système d'écriture proposé pour ces textes religieux $\mathrm{s}^{\prime}$ inscrit dans la droite ligne des traditions orthographiques de la langue française, langue des curés et autres missionnaires. Or, il existe une énorme différence entre les systèmes des sons des langues africaines et ceux des langues européennes. Il résulte de ce fait que les transcriptions proposées semblent être tout simplement des approximations des systèmes phonético-phonologiques réels de ces langues. Ainsi que l'écrit Kwenzi-Mikala (1988 : 207), « la transcription cohérente d'une langue suppose en effet qu'on en ait préalablement établi la phonologie ».

Après les indépendances, la majorité des langues africaines dans le monde francophone comme dans le monde anglophone ne sont vraiment pas écrites. La prise de conscience aussi bien des linguistes que des décideurs africains du fait que le développement culturel est inséparable de celui des langues amena l'organisation des grandes réunions, à l'échelle continentale comme à l'échelle nationale selon les pays, de concertation autour de la transcription des langues africaines. De toutes ces concertations sont nées la plupart des systèmes actuels dans les langues africaines. 


\section{L'ETABLISSEMENT DES ALPHABETS ET DES SYSTEMES DE TRANSCRIPTION}

A l'initiative de l'UNESCO ou avec son aide, bon nombre de séminaires, sessions et autres ateliers réunissant de grands spécialistes de la linguistique africaine ont été organisés dès les années 1960 pour concevoir et établir des systèmes d'écriture des langues africaines. Mais ce processus concernait beaucoup plus les pays francophones qui venaient à peine d'accéder à la souveraineté internationale.

A cette échelle, l'on cite entre autres les réunions suivantes comme les plus importantes:

- la réunion d'experts pour l'unification des alphabets des langues nationales de l'Afrique de l'ouest à Bamako en 1966 ;

- la réunion du même genre sur les langues bantu à Yaoundé en 1970 ;

- la réunion de Cotonou en 1975 sur l'harmonisation et la standardisation des alphabets de la sous région comprenant le Bénin, le Burkina-Faso, le Ghana, le Niger et le Togo ;

- et la réunion d'experts sur la transcription et l'harmonisation des langues africaines de Niamey en 1978, visant à établir un Alphabet Africain de Référence

A propos de la réunion de Bamako et celle de Niamey, Touré (1990 : 58) écrit :

«Considérées comme historiques dans le processus de développement des langues africaines et dans la mesure où pour la première fois les linguistes africains et ceux d'autres continents se rencontraient pour élaborer ensemble un système commun de transcription de six langues de l'Afrique de l'Ouest, ces réunions s'imposaient après le Congrès de Téhéran où venait d'être adopté le Programme Expérimental Mondial d'Alphabétisation (PEMA) ».

Lors de ces deux réunions, les experts internationaux mirent au point l'alphabet africain de référence ci-dessous. 


\section{Aa $a \propto B b$ Bb Cc $\underset{C}{C}$$$
\text { Dd Dd } Đ d \text { Dd Do }
$$$$
\text { Ee } \varepsilon \varepsilon \exists \partial F f \digamma f f
$$$$
\text { Gg } \times \times H h H h I \text { i I I }
$$$$
\text { JjKKKKLI Mm Nn J门 }
$$

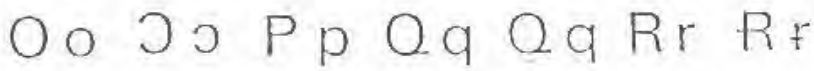$$
\text { Ss Ss } \iint T t T t T f F t
$$

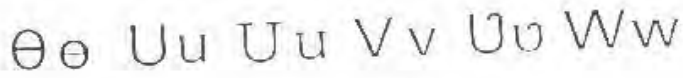$$
X X X X Y Y Y Y
$$$$
\mathrm{ZzZzZz} 33
$$$$
? !(\text { ) «» }
$$

Figure 4: Alphabet Africain de Référence

Touré (1990: 58) ajoute que cet alphabet proposé à Niamey a tenu compte des expériences des pays anglophones cités plus haut: "cette expérience a été extrêmement utile pour les nouveaux engagés dans une politique de promotion de leurs langues".

L'on peut également observer que c'est l'alphabet qui tient compte des signes de l'Alphabet Phonétique International (API) régulièrement aménagé aux besoins particuliers de tel groupe de langues, mais surtout des propositions de l'alphabet Africa élaboré par l'International African Institute pour la transcription phonétique spécifique des langues africaines. On peut par exemple voir que les consonnes [dz], [t $\int$ ] et la semi-consonne palatale [j] de l'API sont respectivement transcrites et/ou orthographiées $j, c$ et $y$. L'on peut aussi observer la prise en compte des spécificités majeures des langues ouest-africaines telles que les interdentales $(\partial, \theta)$, les 
implosives $(6, d)$ et les rétroflexes $(\mathcal{d} t)$ entre autres. Et la voyelle $[y]$ de l'API est représentée par le symbole de l'approximante $v$ alors que les voyelles [ $\varepsilon$ ] et [ə] sont notées telles quelles.

Les visées orthographiques de cet alphabet sont clairement manifestes par la présence des signes pour la ponctuation exprimant l'interrogation (?), l'exclamation (!), les parenthèses, les guillemets («»), la virgule (,), le point-virgule (;) et le point final (.). L'on note également la distinction entre les formes majuscules et les formes minuscules.

L'alphabet africain de référence fait également une part belle à la notation des tons des langues (tons haut, bas, montant, descendant et moyen) et de la nasalisation ( $)$. Le dévoisement (par un point souscrit) et la centralisation (par le tréma) des voyelles sont également pris en compte. Les consonnes sourdes sonorisées $(\underline{c}, \underline{h}, \underline{s}$ et $\underline{\mathrm{t}}$ ) comme les consonnes sonores dévoisées (ㅁ$)$ sont aussi représentées.

Cependant, la tenue considérable de détails phonétiques - largement au-delà des faits proprement phonologiques - laisse quelque peu voir que cet alphabet est tout simplement la transcription phonétique, au sens proprement scientifique, du système des langues de l'Afrique de l'ouest, de telle sorte qu'il ne saurait avoir une grande différence entre l'orthographe des textes (littérature, courriers écrits, circulaires administratifs, etc.) de ces langues et les descriptions linguistiques de ces langues sur le plan scientifiques. La remarque est que cet alphabet ne semble pas tenir compte du public profane des sciences du langage.

Il faut reconnaître que l'échec de cet alphabet (Coulmas 1996) repose non seulement sur l'excès de scientificité qui le caractérise mais aussi sur bon nombre de faiblesses des politiques linguistiques de nombreux pays africains. Touré (1990 : 58), à propos de la République de Guinée, explique cet échec dans les termes suivants:

«L'absence de toute phase d'explication et de sensibilisation, l'insuffisance des programmes de formation des maîtres ou de production de manuels pédagogiques, et somme toute une politique linguistique inadéquate aux réalités socio-culturelles et économiques, expliquent les résultats de ce pays dans un domaine où le souci de la recherche et de la planification, doit aller de pair avec celui de l'action ».

Cette image de la situation en Guinée est typique au problème d'écriture des langues africaines dans la majorité des pays d'Afrique subsaharienne.

A la suite de Bamako (où furent définis les principes et orientations à suivre par tous les pays représentés), est née une dynamique amenant plusieurs pays à 
prendre des dispositions officielles pour l'écriture de leurs langues. Le même Touré (1990 : 58) souligne ensuite :

« Entre autres le Niger qui, par l'arrêté nº17/MEN alpha du 27 avril 1966 et le Mali qui, par le décret n⿳085PG/RM du 26 mai 1967, ont fixé l'alphabet et l'orthographe de leurs langues nationales... Le Sénégal, le Burkina Faso, le Bénin ont également pris des mesures officielles en faveur de la transcription de leurs langues... La Guinée a été le pays qui a montré une réelle volonté politique dans ce domaine. Dans ce pays, l'adoption d'un alphabet national a été immédiatement suivie de la décision d'introduire les langues guinéennes dans le système éducatif $\gg$.

Il faut toutefois noter que quelques divergences peuvent être remarquées entre les alphabets particulièrement adoptés par les différents pays. Par exemple, tandis que Bamako recommandait, au nom du principe d'un seul signe pour un seul son, l'usage de lettres barrées, certains pays choisirent de représenter les mêmes sons par des digraphes. Un certain nombre de divergences sont également celles apparues entre les systèmes choisis par les pays anglophones et ceux des pays francophones en ce qui concerne l'écriture de voyelles ouvertes.

Ce qui a nécessité la réunion de Niamey qui a permis non seulement de faire le bilan des différentes expériences mais en outre de mieux clarifier les principes d'écriture des langues africaines.

Enfin, il faut reconnaître que nombre de pays aujourd'hui ont utilisé l'Alphabet Africain de Référence soit pour élaborer soit pour améliorer leur alphabet national. Mais il reste à intégrer les alphabets nationaux non seulement en des systèmes d'orthographe adéquats aux réalités socio-économiques modernes mais aussi dans la conscience de la masse populaire des différents pays.

\section{LA GRAPHIE DU MOT DANS LES LANGUES AFRICAINES}

En ce qui concerne l'orthographe des mots, il existe en général deux traditions, à savoir : le système conjonctif et le système disjonctif. Dans le premier les mots sont représentés selon une structure interne complexe. Le mot peut ainsi constituer un ensemble écrit qui contient par exemple un préfixe verbal, un formatif, une racine et des extensions. Ce qui peut donner, dans une langue comme le yilumbu, un mot du type:

(1) Batsiwenda «ils sont partis »

Ba - préfixe verbal «ils»

- tsi - formatif de temps« passé proche» 
+ wend - racine verbale « partir»

- a finale verbale

Dans cette forme d'écriture l'on ne tient absolument pas compte de la relation entre le mot de langue africaine et le mot de langue européenne dans laquelle est faite la traduction. Ce qui pose par ailleurs le problème de la détermination et définition du mot. Dans l'exemple (1) ci-dessus l'on peut observer que si batsiwenda constitue un mot lumbu selon l'écriture conjonctive, sa traduction française constitue cependant une phrase entière. Ceci pose également des problèmes de morphosyntaxe dans les langues africaines. On peut de même poser la question: qu'est-ce qui constitue le mot? La réponse d'une telle question comme celles des problèmes sus évoqués nécessite le développement étudié de l'orthographe de la langue. De manière générale, l'orthographe du mot est fonction de la notion qu'on a du mot lui-même.

Les problèmes posés par la conjonction des morphèmes dans l'orthographe des langues africaines semblent trouver de solutions dans une écriture disjonctive. L'écriture conjonctive est la forme graphique du mot dans sa structure interne plus simple. Ce qui signifie que les formatifs sont notés séparément du radical verbal.

Depuis l'époque des missionnaires et administrateurs coloniaux, bon nombre de langues africaines ont souvent été écrites de façon disjonctive. Cette attitude trouve son origine dans l'habitude qu'ils avaient de leurs langues maternelles respectives qui sont pour la plupart l'anglais, le français, l'espagnol et dans une moindre mesure le portugais. L'on note que ces langues ont toutes une écriture disjonctive généralement héritée de l'orthographe latine.

Pour certaines langues africaines comme le zulu (Poulos \& Msimang 1998 : 8), les missionnaires et autres grammairiens, qui ont été dès le début du XIXème les premiers à transcrire orthographiquement la langue, ont eut à penser qu'il y avait une relation significative nette entre le mot zulu et le mot anglais. Ils supposèrent ainsi que chaque formatif constituait un mot distinct suivant le système anglais avec des exemples ci-après:

(2) Ba ya sebenza They are working «ils travaillent »

E khaya At home «à la maison»

Ba zo fika They will come « ils viendront»

Il fallut attendre la fin du siècle pour que l'on réalise que le mot zulu était bien plus complexe que celui de l'anglais. En effet, là où le mot anglais consiste habituellement en un morphème libre (un seul morphème constituant un seul mot) le mot zulu pouvait être un ensemble de morphèmes agglutinés. Ces remarques 
ont amené Colenso (1882) 5 à faire usage pour la première fois du conjonctivisme pour l'orthographe du zulu qu'il définit en termes d'accentuation de la syllabe pénultième. Les items en (2) sont finalement notés comme en (3).

(3) Bayasebe:nza «ils travaillent»

Ekha:ya «à la maison»

Bazofika «ils viendront »

La pertinence du choix de l'écriture conjonctive comme présenté ci-dessus pour le zulu est le fait de la prise en compte des conditions phonologiques pour l'identification du mot. Pour le cas du zulu qui vient d'être évoqué le mot est identifiable phonologiquement par l'accent principal (se traduisant par une longueur vocalique) qui tombe sur la syllabe pénultième (Poulos \& Msimang 1998 : 9).

Cependant, en dehors de l'approche phonologique pour l'identification du mot il existe également différentes approches aussi bien au niveau syntaxique qu'au niveau morphologique. Le choix entre le conjonctivisme et le disjonctivisme nécessite donc comme préalable, une identification réelle de ce qu'est le mot dans la langue. Si par exemple l'on revient sur le critère de la longueur vocalique de la syllabe pénultième du zulu, il se pose d'énormes questions qui ne trouvent aucune réponse sur le plan phonologique.

En effet, la longueur de la syllabe pénultième dépend fortement de la position en isolation ou en contexte de phrase (ou groupe de mots). Si en isolation la longueur est admise dans le mot, en contexte de phrase (ou groupe de mots) seul le dernier mot de la phrase a une longueur sur la syllabe pénultième. Cela implique-il qu'une phrase (ou groupe de mots) comprend un mot unique? D'une part, une phrase interrogative qui n'a habituellement pas de longueur sur la syllabe pénultième, particulièrement si la phrase termine avec l'adjonction na? Dirions-nous dans ce cas que la phrase interrogative ne consiste en aucun mot? D'autre part, la longueur vocalique est généralement la caractéristique des idéophones, des démonstratifs et des interjectifs. Va-t-on les considérer comme les seuls mots véritables de la langue $\mathrm{du}$ fait de l'allongement vocalique.

Finalement, le mot est bel et bien une réalité à la fois phonologique, morphosyntaxique, sémantique et même pragmatique. La notion du mot dans le cadre de l'établissement d'un alphabet trouve toute sa pertinence dans l'idée selon laquelle l'alphabet est constitué des symboles qui, mis ensemble, forment des mots. Par conséquent, le processus de confection d'un alphabet pour l'orthographe des langues africaines nécessite $q u^{\prime}$ on définisse concrètement la notion du mot.

5 Cité par Poulos \& Msimang (1998: 9). 
En ce qui concerne les langues africaines d'une manière générale, il faut considérer qu'elles soient de différentes familles linguistiques. De fait, elles présentent une diversité d'organisations syntaxiques, morphologiques et phonologiques. Ainsi, l'écriture ou l'orthographe du mot ne peut être standardisé qu'à l'intérieur de chaque famille linguistique. Et même, au sein d'une même famille on observe certaines différences. C'est le cas par exemple des 9 langues africaines officielles d'Afrique du Sud (ndebele sud, sotho nord, swazi, tsonga, tswana, venda, xhosa et zulu). Ces langues sont toutes des langues bantu, mais la division du mot diffère selon les groupes de langues. Tandis que les langues du groupe nguni (ndebele, swazi, xhosa et zulu) ont généralement une écriture conjonctive, les langues du groupe sotho-tswana et autres groupes s'écrivent par contre de manière disjonctive. Ce qui se dégage des exemples ci-après pour les expressions déjà énoncées en (2) et (3).

(4) Groupe Nguni:

Langue : Zulu cf. (3) (Ecriture conjonctive)

Ba- (PV) -ya- (Fo1) -se- (Fo2) -benz-a (Rad + Fi) ${ }^{6}$

e- (PNL) -kha:ya (Rad)

ba- (PV) -zo- (Fo) -fik-a (Rad + Fi)

Langue : Xhosa (Ecriture conjonctive)

Bayasebenza

ba- (PV) -ya- (Fo1) -se- (Fo2) -benz-a ( Rad + Fi) « ils travaillent »

Ekhaya

e- (PNL) -khaya (Rad) «à la maison»

Bazakufika

ba- (PV) -za (Fo) -ku- (PN, cl.15) -fik-a ( $\mathrm{Rad}+\mathrm{Fi})$ «ils viendront »

Langue : Swati (Ecriture conjonctive)

Baya sebenta

ba- (PV) -ya (Fo) sebent-a ( Rad + Fi) «ils travaillent »

Ekhaya

e- (PNL) -khaya (Rad) «à la maison »

Batao fika

ba- (PV) -ta- (Fo1) -o (Fo2) fik-a ( Rad + Fi) «ils viendront »

(5) Groupe Sotho-tswana

Langue: Sesotho (Ecriture disjonctive)

Ba ea sebetsa

ba $(\mathrm{PV})$ ea $(\mathrm{Fo})$ sebetsa $(\mathrm{Rad}+\mathrm{Fi})$ « ils travaillent »

Hae $\quad \varnothing(\mathrm{PNL})$ hae $(\mathrm{Rad})$ «à la maison »

$6 \mathrm{PV}=$ préfixe verbal $; \mathrm{Fo}=$ formatif $; \mathrm{Rad}=$ radical $; \mathrm{Fi}=$ finale $; \mathrm{PNL}=$ préfixe nominal locatif $; \mathrm{SL}=$ suffixe locatif. 
Ba tla khutla

ba (PV) tla (Fo) khutla ( $\mathrm{Rad}+\mathrm{Fi})$ « ils viendront »

Langue : Tswana (Ecriture disjonctive)

Ba mo tirong

ba (PV) mo (Fo) tirong (Rad) «ils travaillent »

Ko gae

ko (PNL) gae (Rad) «à la maison»

Ba tla tla

ba (PV) tla (Fo) tla (Rad) «ils viendront »

Langue : Sepedi (Ecriture disjonctive)

Ba ya bereka

ba $(\mathrm{PV})$ ya $(\mathrm{Fo})$ bereka $(\mathrm{Rad}+\mathrm{Fi})$ «ils travaillent »

Ko gaye

ko $(\mathrm{PNL})$ gaye $(\mathrm{Rad})$ «à la maison »

Ba ye kla

ba (PV) ye (Fo) kla (Rad) «ils viendront »

(6) Autre groupe de langues

Langue : Tsonga (Ecriture quelque peu mixte)

Va tirha

va $(\mathrm{PV})$ tirha $(\mathrm{Rad}+\mathrm{Fi})$ « ils travaillent »

Ekaya

e- (PNL) +kaya (Rad) «à la maison»

$\mathrm{Va}$ ta ta

va $(\mathrm{PV})$ ta $(\mathrm{Fo})$ ta $(\mathrm{Rad})$ «ils viendront»

Langue : Venda (Ecriture quelque peu mixte)

Vha khou shuma

vha $(\mathrm{PV})$ khou $(\mathrm{Fo})$ shuma ( $\mathrm{Rad}+\mathrm{Fi})$ «ils travaillent »

Hayani

haya- (Rad) -ni (SL) «à la maison »

Vha do da

vha $(\mathrm{PV})$ do $(\mathrm{Fo})$ da $(\mathrm{Rad})$ «ils viendront »

\section{REMARQUES:}

En dehors du fait qu'elles sont toutes des langues bantu regroupées en familles, l'on remarque que dans les langues sud-africaines leur graphie ne tient pas compte des leurs systèmes tonals. En outre, tandis que les langues nguni et les langues sotho sont de manière stricte respectivement conjonctives et disjonctives, les autres langues tels que le tsonga et le venda semblent combiner les deux systèmes. 
En définitive, comme le fait remarquer Van Wyk (1995 : 83-84), le choix entre le conjonctivisme et disjonctivisme est purement et simplement une question de convention orthographique. Dans la plupart des langues africaines, il n'y a pas eu beaucoup de réflexions sur la question de la division du mot. Nombre de langues, comme le xhosa (RSA $\left.{ }^{7} 1972\right)$, ont tout simplement adoptées la forme héritée soit des missionnaires soit de la tradition des langues européennes.

\section{EN CE QUI CONCERNE L'ECRITURE DES LANGUES GABONAISES}

De nos jours le développement des langues est intimement lié à leur écriture. Il est certainement évident que le développement des langues gabonaises passe préalablement par leur écriture. Il ne saurait par exemple y avoir de manuel d'apprentissage et d'enseignement si un alphabet et un système d'orthographe ne sont pas définitivement élaborés, adoptés et véhiculés. La confection des dictionnaires nécessite également une forme standardisée de l'orthographe des langues. La section suivante qui commence par un état des lieux sur l'écriture des langues gabonaises fait une analyse des alphabets déjà existants pour l'écriture de ces langues.

\section{D'ABORD UN ETAT DES LIEUX}

Dans son article sur l'histoire des langues du Gabon, Mayer (1990) suggère sommairement trois importantes périodes: l'écriture exploratoire, l'écriture pédagogique et l'écriture scientifique.

La première période correspond à l'arrivée des premiers européens sur les côtes gabonaises, et particulièrement dans l'Estuaire et dans la région nord de l'ancien royaume de Loango (de Mayumba au Cap Lopez), et celles des explorateurs (en majorité des Portugais). Elle se situe entre le début du XVIème siècle et la deuxième moitié du XIXème. Le système d'écriture de cette période est totalement celui des langues européennes. Mayer (1990 : 67) indique que cette longue période, qui est la plus ancienne écriture de nos langues, a fourni aux langues gabonaises «trois siècles de traces écrites ».

Ces traces sont essentiellement reportées sur des cartes «qui portent les premières mentions de noms de peuples ou de noms de lieux». En dehors des ethnonymes, toponymes et hydronymes, ces écrits concernent aussi des noms de personnes (généralement de noms de personnages illustres, e.g. Mani Pongo, Mani Gabam, Rapontchombo, etc.) et de noms communs. De cette période l'on retient l'orthographe de mots comme:

7 RSA est mis pour Republic of South Africa. 


$\begin{array}{ll}\text { (7) Bramas } & \text { pour Bavarama } \\ \text { Pongoé } & \text { pour Mpongwe } \\ n^{\prime} l a & \text { pour dire tête en fang } \\ \text { land'jogg } & \text { pour dire trompe (d'éléphant) en fang } \\ \text { Gobbi } & \text { pour Ngubi (Ngowe) } \\ \text { Majumba } & \text { pour Mayumba } \\ \text { Commi } & \text { pour Nkomi }\end{array}$

Il faut noter que ces premières mentions « comportent de nombreuses erreurs dues aux incompréhensions et aux malentendus, et plus fondamentalement à la différence des systèmes phonologiques des langues pratiquées » (Mayer 1990 : 67).

La deuxième période qui concerne une écriture dite pédagogique se rapporte à l'intérêt porté par les Occidentaux sur les langues africaines. Cette phase, à partir de 1850, inaugure l'apprentissage et la scolarisation de ces langues. Les missionnaires enseignent la lecture et l'écriture non seulement aux locuteurs natifs, mais aussi à tous ceux qui, Occidentaux comme locuteurs d'autres langues, s'y intéressent. L'écriture se systématise, des imprimeries voient le jour et des ouvrages sont publiés. Il s'agit essentiellement de manuels, grammaires, syllabaires, opuscules et des dictionnaires le plus souvent monolingues et "parfois abondamment et magnifiquement illustrés » (Mayer 1990: 73). C'est aussi la période, largement dominée par les travaux des missionnaires, des premières traductions des textes liturgiques et bibliques. L'un des faits marquant de cette période est la véhicularisation de certaines langues par le biais des publications missionnaires. L'on note ainsi :

(8) Le ndumu dans le sud-est

Le yipunu et le yisangu dans le sud

Le yinzebi et le liduma dans l'est

Et le fang dans l'ensemble nord (de l'ouest à l'est)

Pour Mayer (1990: 73), «l'apprentissage formel des langues a été abandonné aujourd'hui, mais la période pédagogique survit sous la forme de livrets de cantiques écrits dans les langues du Gabon ». Cependant, l'alphabet et le système d'écriture des langues gabonaises demeurent dans cette période ceux empruntés aux langues européennes.

La troisième phase établie par Mayer (1990: 88-89) est celle qui prend vraiment corps dès la seconde moitié du XXème. Elle se caractérise par la mise en valeur et l'usage des spécificités phonético-phonologiques des langues gabonaises pour l'établissement des systèmes d'écriture cohérents. Ceux-ci résultent des études linguistiques plus ou moins scientifiques. Parmi les travaux scientifiques de la

$8 \quad$ Ces exemples sont extraits de Mayer (1990 : 68-71). 
première heure, l'on cite Raponda-Walker qui dès 1932 proposa un alphabet des langues gabonaises.

Dans son article paru dans le Journal de la Société des Africanistes (RapondaWalker 1998 : 7-15) Raponda-Walker donne un aperçu analytique d'un alphabet ${ }^{9}$ pour la transcription des langues du Gabon s'insurgeant ainsi contre la double notation alphabétique qui est faite des langues gabonaises:

- l'usage de l'alphabet anglais par les missionnaires protestants américains

- et l'usage de l'alphabet français par les missionnaires catholiques

Finalement, la période scientifique telle qu'élucidée par Mayer est celle qui se poursuit jusqu'à ce jour où des travaux et discussions sont régulièrement abordés en vue de doter les langues gabonaises d'un système d'écriture normalisé qui s'impose. La plupart des propositions alphabétiques s'inspirent largement de l'Alphabet Phonétique International (API) et de l'Alphabet Africa élaboré par l'International African Institute pour la transcription particulière des langues africaines.

Aussi, il ne faut pas perdre de vue, ainsi que le souligne Mayer (1990: 66) que «les périodes se chevauchent et qu'il y a parfois stagnation voire recul. En outre, à chaque nouvel arrivant au Gabon, et même pour les nationaux qui n'ont pas reçu d'apprentissage à l'écriture et à la lecture des langues ethniques en plein XX̀̀ siècle, recommence une écriture exploratoire... Il serait illusoire d'enfermer chaque période dans une datation absolue, car ce serait ignorer ces retours en arrière et le fait que les trois formes d'écriture - exploratoire, pédagogique et scientifique - peuvent en définitive coexister ».

L'on retient du récit de Mayer (1990) que le passage au Gabon des langues parlées aux langues écrites remonte à l'époque coloniale. Toutefois, comme le soulignent Ndinga-Koumba-Binza \& Roux (2009 : 86), cette présentation historique en termes de périodes est peu satisfaisante. En effet, s'il faut tenir compte du fait de l'écriture en tant que communication graphique diverse, l'on dira que bon nombre de langues gabonaises ont une expression graphique depuis nos ancêtres.

Les peintures rupestres trouvées dans certaines grottes telles que celles de Bongolo (par exemple la grotte de Muvindu) à Lébamba (province de la Ngounié), les incisions scarificatrices ou encore la variété des masques des différentes ethnies et les différentes gravures et peintures (parfois sur le corps) dans les différents rites religieux traditionnels (Bwiti, Ndjembe, Njobi, Mungala, etc.) sont des témoins d'un début de forme d'écriture dans nos langues à l'image de celle de Mésopotamie.

9 Je reviens sur l'analyse de l'alphabet de Raponda-Walker dans les pages suivantes. 
Ndinga-Koumba-Binza \& Roux (2009 : 86) indiquent que la prise en compte de ces quelques considérations spécifiques revèle que trois ères peuvent être identifiées en ce qui concerne l'écriture des langues gabonaises. Il s'agit de :

(i) l'ère ancestrale,

(ii) l'ère coloniale, et

(iii) l'ère moderne.

Ndinga-Koumba-Binza \& Roux (2009 : 86) résument les trois ères dans le tableau ci-après.

\begin{tabular}{|c|l|l|l|}
\hline \multirow{2}{*}{$\begin{array}{c}\text { Ere } \\
\text { Ancestrale }\end{array}$} & \multirow{2}{*}{ Ere Coloniale } & \multicolumn{2}{|c|}{ Ere Moderne } \\
\cline { 3 - 4 } & & \multicolumn{1}{|c|}{ Administrative } & Scientifique \\
\hline$?$ & Pongoé & Mpongwe (ethnonyme) & Mpongwe \\
\hline$?$ & N'la (Fang) & Nla “tête" & Nla \\
\hline$?$ & Land'jogg (Fang) & Lang Nzoghe "trompe"(éléphant) & Lan Nzoghe \\
\hline$?$ & Majumba & Mayumba (toponyme) & Mayumba \\
\hline$?$ & Commi & Nkomi (ethnonyme) & Nkomi \\
\hline
\end{tabular}

Tableau 1: $\quad$ Trois ères d'écriture des langues gabonaises

L'ère coloniale comprends les deux premières périodes de la classification de Mayer (1990). Elle commence exactement avec les écrits des explorateurs. Comme précédemment $\mathrm{vu}$, la forme d'écriture moderne latine apparaît dans nos langues avec l'arrivée des missions évangéliques catholiques et protestantes et de l'administration coloniale. Ces tentatives vont des traductions des documents religieux (Bible, catéchisme, cantiques, etc.) aux transcriptions des noms locaux (noms de personnes, de villages, de peuples et ethnies, etc.).

Malgré cette situation pratiquement séculaire nos langues sont toujours considérées comme des langues à tradition orale comme si elles n'avaient jamais eu de forme scripturaire. Même s'il est vrai que leur écriture ne fut pas tout à fait standard étant donné la diversité de systèmes due le plus souvent à l'origine (religieuse ou linguistique) des transcripteurs.

Pour Alexandre (tel que cité par Kidda Awak 1990:11) la langue fang a acquis "trois transcriptions [catholiques] et deux [protestants] pour environ un million de locuteurs, sans parler des différences morphologiques dues à des calques catholiques sur l'allemand, le français et l'espagnol, et protestants, sur l'américain ».

L'ère moderne comprend la troisième période de la classification de Mayer. C'est l'ère non seulement des études linguistiques systématiques, aussi celle de la conscience nationale et des mouvements nationalistes. C'est également l'ère de la première expression de la revalorisation des langues et cultures locales. On voit également s'exprimer une certaines volonté politique par l'organisation des 
séminaires, conférences et ateliers, et éventuellement la création au sein de l'université nationale en 1994 d'un département en vue de la formation des linguistes. C'est enfin l'ère de la majorité des propositions, des alphabets et systèmes d'orthographe conçus pour l'écriture des langues gabonaises.

\section{ECRITURE DES MISSIONNAIRES ET LINGUISTIQUE GABONAISE ACTUELLE}

Le système d'écriture des missionnaires a récemment suscité des débats, d'abord lors de la Table Ronde sur les Politiques Linguistiques et l'Enseignement des Langues Gabonaises en 1997, puis lors de la Session de Concertation sur l'Orthographe des Langues du Gabon en 1999. Pour Mayer (1990: 88), «ce serait une belle erreur historique de croire que l'approche scientifique de l'écriture des langues ethniques a fait suite à une série d'approximations et - pourquoi ne pas le dire - de 'bricolages' qui aurait résulté d'une certaine insuffisance intellectuelle ».

La linguistique gabonaise contemporaine a une considération peu importante des travaux des missionnaires dans le processus de développement des langues gabonaises. Ces travaux des missionnaires ne sont pas poursuivis là où ils se sont arrêtés. C'est le cas par exemple des travaux de Raponda-Walker qui, à défaut d'être repris et améliorés par des linguistes, font l'objet d'édition et réédition (sans aucune révision) par une Organisation Non-Gouvernementale qui a à cœur le développement des langues et cultures gabonaises : la Fondation Raponda-Walker.

Il a été par ailleurs démontré que les pays qui ont tout simplement adopté les orthographes élaborés par les administrateurs coloniaux et les missionnaires pour l'écriture des langues africaines ont moins connu les problèmes d'orthographe et d'alphabet qui s'illustrent au Gabon (McLaren 1936, Poulos \& Msimang 1998). La continuité de leurs travaux n'aurait pas laissé la linguistique gabonaise au stade présent, ne serait-ce qu'en ce qui concerne l'orthographe et l'écriture des langues gabonaises. Car l'on sait que «les tentatives d'écriture des langues gabonaises remontent à l'arrivée des premiers colons » (Idiata 2002: 48) et que les premiers à enseigner ces langues à l'école avec un système d'orthographe accessible aux populations furent ces missionnaires énormément critiqués de nos jours pour bon nombre de maladresses qu'ils n'avaient peut-être pas pu éviter. Car la science linguistique elle-même a considérablement évolué pendant le siècle dernier et les outils méthodologiques utilisés aujourd'hui n'étaient pas encore maîtrisés au début du XXème siècle.

Par ailleurs, c'est assurément en termes de blâme qu'Idiata (2002 : 59) écrit:

«Alors que les projets de développement des langues sont rarissimes, et que l'insertion de ces langues à l'école dans les établissements 
publics demeure, à ce jour, à sa phase théorique, ou presque, le débat a porté uniquement sur les systèmes d'écriture... ».

Ce débat, Idiata lui-même le qualifie de "vrai faux débat qui n'a pas beaucoup de pertinence aujourd'hui». Pour ma part, le débat existe parce que d'une part le système d'écriture des missionnaires n'avait pas été tout simplement continué et adapté aux principes actuels, et d'autre part parce qu'un alphabet et un système d'orthographe pour les langues gabonaises restent encore à être adoptés par les pouvoirs publics et vulgarisés au sein de la population.

Je me demande si l'alphabet romain utilisé différemment par bon nombre de langues européennes (français, anglais, espagnol, allemand, néerlandais, etc.) répond ne fut-ce qu'à 50\% des critères de scientificité que l'on pourrait concevoir dans le cadre de l'élaboration d'un alphabet et à l'établissement d'une orthographe. Et pourtant ces langues européennes ont parfois des systèmes phonético-phonologiques bien plus complexes que ceux de nos langues bantu.

L'on pose aussi souvent le problème de la notation des tons. Il est vrai qu'une langue bantu sans ton est inimaginable. Et c'est à ce niveau que nos langues diffèrent vraisemblablement des langues européennes. Cependant, si cela était adopté les langues gabonaises ne seraient absolument pas les seules langues bantu au monde à s'écrire sans notation des tons. Elles courent par contre le risque d'être parmi les rares langues bantu à avoir une notation tonale dans l'orthographe et en dehors d'une pure description phonétique ou phonologique ou encore tonologique. Comme quoi, science conscience n'est que ruine de l'âme.

Car c'est finalement à la ruine que conduisent le vrai faux débat et la polémique sur la notation des tons. De l'Afrique du Sud en Tanzanie et au Kenya, toutes les langues de cette région - le zulu, le xhosa, le tswana, le sepedi, le venda, le tsonga, le ndebele, le sotho, le shona, le herero, le lingala, le ciluba, le swahili, etc. s'écrivent sans aucune notation tonale avec une adoption parfois des alphabets des colonisateurs et missionnaires. Et cela n'empêche ni la lecture, ni l'enseignement, ni les littératures poétiques, romanesques et orales de ces langues.

Pour ma part, j'estime qu'il nous faut absolument éviter cette attitude à vouloir recréer le monde. C'est cette attitude que fustige Mouguiama-Daouda (1997: 169) lorsqu'il écrit:

«En effet, les chercheurs africains ont tendance à analyser les faits linguistiques sans référence à un cadre théorique général. Ainsi, aucun rapprochement n'est fait avec les phénomènes identiques ou analogues attestés ailleurs. Une telle attitude conduit, au mieux, à enfoncer des portes ouvertes, au pire, à avoir une vision erronée, parce que trop individualisée, de la vérité scientifique». 
Enfin de compte, le blocage au développement des langues n'est pas seulement la décision politique qui est par ailleurs citée, mais aussi et surtout la décision linguistique qui devra se matérialiser par beaucoup d'unanimité dans la linguistique gabonaise et par l'importation dans celle-ci des technologies, des théories et des conceptions qui ont fait leurs preuves et tiennent la route sous d'autres cieux. Il va sans dire que toute chose reste réadaptable aux réalités gabonaises si besoin s'impose.

Il faut également reconnaître que si à l'époque des missionnaires dès le XIXème, les langues africaines, en dépit de l'usage des caractères de l'alphabet latin, étaient dotées de systèmes d'écriture (Touré 1990: 56) c'est que le niveau de développement actuel des langues gabonaises est suffisamment inférieur à celui qu'il pouvait être il y a cent ans. Les propos suivants de Mayer (1990: 66) en sont une parfaite illustration:

«Parmi la cinquantaine d'ouvrages que m'avait dévoilés le P. POUCHET, figuraient grammaires, des dictionnaires et bien entendu des traductions de catéchismes et de textes bibliques, le tout dans une diversité insoupçonnée de langues du territoire national. Mais ce qui m'avait frappé dans ce lot d'ouvrages, c'était la présence de quelques vieux abécédaires et syllabaires du début du siècle. C'étaient des manuels liés non pas à l'enseignement $\mathrm{du}$ français mais à l'enseignement des langues locales. Ce sont de précieux témoins d'un enseignement systématisé que les jeunes générations ont oublié aujourd'hui».

Toutefois, l'adoption d'un alphabet reste un préalable nécessaire à tout développement que peuvent connaître les langues gabonaises. A ce sujet des tentatives pour doter ces langues d'un système d'orthographe ont été faites à plusieurs reprises.

\section{LES ALPHABETS DES LANGUES GABONAISES EXISTANTS}

Il faut reconnaître que la toute première tentative de mise en place d'un alphabet pour les langues gabonaises est celle des missionnaires. Mais comme vu précédemment, ce fut tout simplement une adoption des alphabets des langues européennes selon que les missionnaires étaient anglophones ou francophones.

Depuis lors des efforts ont été fournis pour pouvoir élaborer un alphabet adéquat aux langues gabonaises en tenant compte de leurs spécificités phonéticophonologiques. De fait, il existe à ce jour quatre propositions d'écriture des langues gabonaises. 
Une première liste de ces propositions est faite par Idiata (2002). Ndinga-KoumbaBinza \& Roux (2009) ont procédé à une évaluation de celles-ci avec une attention particulière sur la représentation des tons, de la longueur vocalique, de la ponctuation et de la division du mot. Cette évaluation montre qu'il y a un certain nombre de paramètres à considérer dans l'élaboration d'une orthographe pour les langues gabonaises. Je reviens ici sur certains de ces paramètres.

\section{L'ALPHABET ORTHOGRAPHIQUE DE RAPONDA-WALKER}

La première tentative remonte à Raponda-Walker. Dans cet article intitulé «Alphabet des idiomes gabonais » (réédité en 1998 par la Fondation Raponda-Walker) paru pour la première fois en 1932 dans le Journal de la Société des Africanistes, Raponda-Walker établit la liste de phonèmes et suggère les graphèmes correspondants en s'inspirant à la fois de l'API et de l'alphabet Africa. Il propose un système de 36 signes alphabétiques - dont certains sont affectés de diacritiques.

Mayer (1990: 88) et Idiata (2002: 50) divergent quant au nombre des signes alphabétiques proposés par Raponda-Walker. Le premier cite 37 signes tandis que le second parle de 38 signes. Le décompte que j'ai personnellement fait à partir de l'article «L'alphabet des idiomes gabonais » (dans sa réédition de 1998, Les langues du Gabon, par la Fondation Raponda-Walker) présente 36 signes alphabétiques comme relevés dans le tableau 2 ci-après.

On y compte 28 consonnes et 8 voyelles. Les graphèmes sont établis selon le principe qu'un seul signe doit correspondre à un seul son, et un seul son à un seul signe. D’après Mayer (1990: 88), cet alphabet aurait été adéquat pour «la bonne orthographe des langues pratiquées au Gabon ».

\begin{tabular}{|llllll|}
\hline & Voyelles & \multicolumn{3}{c|}{ Consonnes simples } & Consonnes avec diacritiques \\
$\mathrm{a}$ & {$[\mathrm{a}]$} & $\mathrm{b}$ & $\mathrm{l}$ & $\mathrm{v}$ & $\hat{\mathrm{c}}[\mathrm{l}]$ \\
$\mathrm{e}$ & {$[\mathrm{e}]$} & $\mathrm{c}[\mathrm{k}]$ & $\mathrm{m}$ & $\mathrm{w}$ & $\mathrm{\epsilon}[\mathrm{t} f]$ \\
$\mathrm{e}$ & {$[\mathrm{e}]$} & $\mathrm{d}$ & $\mathrm{n}$ & $\mathrm{x}$ & $\dot{\mathrm{g}}[\mathrm{\chi}]$ ou $[\mathrm{y}]$ \\
$\mathrm{i}$ & {$[\mathrm{i}]$} & $\mathrm{f}$ & $\mathrm{p}$ & $\mathrm{y}$ & $\tilde{\mathrm{n}}[\mathrm{n}]$ \\
$\mathrm{o}$ & {$[\mathrm{o}]$} & $\mathrm{g}$ & $\mathrm{q}[\mathrm{k}]$ & $\mathrm{z}$ & $\mathrm{n} \cdot[\mathrm{y}]$ \\
$\ddot{\mathrm{o}}$ & {$[\mathrm{o}]$} & $\mathrm{h}$ & $\mathrm{r}$ & & $\mathrm{r} \cdot \mathrm{x}]$ \\
$\mathrm{u}$ & {$[\mathrm{u}]$} & $\mathrm{j}[\mathrm{dz}]$ & $\mathrm{s}$ & & $\ddot{w}[\mathrm{u}]$ \\
$\ddot{\mathrm{u}}$ & {$[\mathrm{y}]$} & $\mathrm{k}$ & $\mathrm{t}$ & & \\
\hline
\end{tabular}

Tableau 2: Alphabet des langues gabonaises selon Raponda-Walker

Pour Raponda-Walker (1998: 7), «cette orthographe, grâce à des signes diacritiques spéciaux affectant les différentes lettres, reproduit plus facilement la prononciation des mots 
indigènes ». En effet, la liste proposée couvre relativement la majorité des phonèmes présents dans les langues du Gabon.

L'on remarque également dans cette proposition qu'aucune mention n'est faite sur les phénomènes prosodiques et de durée tels que les tons, la longueur vocalique et la gémination. Un fait tout de même important est le souci de l'auteur d'éviter des signes digraphes sur la base l'aphorisme sus-cité, "un seul signe pour chaque son, un seul son pour chaque signe». Il faut aussi noter que ces propositions s'appuient énormément sur l'alphabet des langues latines (Idiata 2002 : 50). Ceci s'observe par la mention des signes $c$, $k$ et $q$ pour le même son [k]. L'on voit également la prise en compte du signe $x$ que l'auteur reconnaît lui-même être inconnu dans les idiomes du Gabon (Raponda-Walker 1998: 14). Cependant, les deux fricatives vélaires sourde $[\chi]$ et sonore $[\gamma]$ sont tous les deux représentés par le signe $\dot{\mathrm{g}}$. Par ailleurs, Raponda-Walker ne fait état ni d'une forme majuscule ni d'une possibilité de ponctuation dans le système qu'il propose.

\section{L'AlPHABET SCIENTIFIQUE DES LANGUES DU GABON (ASG)}

Plus de cinq décennies après Raponda-Walker apparaît l'Alphabet Scientifique des Langues du Gabon (ASG). C'est ici «la seconde tentative d'élaboration d'un système d'écriture spécifique aux langues vernaculaires gabonaises » (Idiata 2002 : 51). L'ASG fut élaboré en 1989 au cour d'un séminaire des experts organisé sous les auspices de l'Agence de Coopération Culturelle et Technique (ACCT, aujourd'hui Agence Internationale de la Francophonie) par le Laboratoire Universitaire de la Tradition Orale (LUTO, aujourd'hui Laboratoire Universitaire de la Tradition Orale et des Dynamiques Contemporaines, LUTO-DC) de l'Université Omar Bongo (UOB).

La nécessité de sa conception réside principalement dans le fait, selon les termes de Martin Alihanga (1990: 17), que «le Gabon cherche à combler son retard en matière d'orthographe des langues nationales ». En effet, "il nous a manqué jusqu'à présent, non seulement les transcriptions scientifiques, mais aussi les ouvrages fondamentaux utiles à la planification de notre effort de conception d'une orthographe scientifique, à savoir l'utilisation de l'alphabet latin avec le minimum de signes simples pouvant servir à la totalité des langues gabonaises » (Alihanga 1990 : 17-18).

En outre, l'ASG a été conçu avec les objectifs suivants:

- Favoriser la recherche sur le patrimoine culturel national, la collecte des documents oraux, leur élaboration, leur analyse contextuelle et leur publication ;

- Créer un alphabet unifié pour toutes les langues du Gabon : la diversité des systèmes d'écriture dans un pays étant considérée comme un facteur d'isolement et un obstacle à l'unité - le but ici étant l'harmonisation adaptée des 
deux systèmes ayant prévalu chez les missionnaires protestants d'une part et catholiques d'autre part ;

- Appuyer les travaux des chercheurs gabonais sur les compétences nationales et internationales ;

- Assurer à l'alphabet national une expérimentation pratique, puis une reconnaissance légale ;

- Trouver une solution pratique aux problèmes de transcription de nos langues Alihanga (1990 : 19).

Il faut également noter que l'ASG a été établi d'après Hombert (1990a: 107) selon les critères suivants :

- La prise en compte de toutes les spécificités phoniques de toutes les langues du Gabon (Hombert 1990b) ;

- L'éloignement le moins possible des symboles de l'Alphabet Phonétique International, de l'alphabet Africa, de l'alphabet africain de référence, ainsi que des expériences d'autres pays africains ;

- L'utilisation d'un minimum de digraphes

- L'utilisation d'un minimum de diacritiques

L'ASG comme présenté dans le Tableau 3 est un système de 29 consonnes simples et 9 voyelles. (Hombert 1990a, Carpentier de Changy et Voltz 1990). La notation des tons est envisagée. 9 tons sont notés : infra-bas (ou très bas), bas, moyen, haut, descendant, montant, haut abaissé, très haut et très haut descendant. Contrairement à la proposition de Raponda-Walker, l'ASG admet des digraphes, généralement pour représenter des formes palatalisées (par exemple ny pour le son [n]), labiovélarisées ( $\mathrm{kw}, \mathrm{tw}$ ) et prénasalisées ( $\mathrm{mb}, \mathrm{nd}, \mathrm{ng})$. Les consonnes occlusives labio-vélaires ( $\mathrm{kp}, \mathrm{gb}$ ) et les consonnes affriquées (pf, bv, ts, dz) sont également représentées sont formes de digraphes. L'ASG envisage aussi la longueur vocalique, elle est transcrite par le redoublement de la voyelle.

L'on note dans cet alphabet la présence de quatre diacritiques tous souscrits.

- Un tilde ( ) pour les segments nasals (voyelles et consonnes);

- Une courbe ( $)$ identifiant la voyelle très brève;

- Un tréma (..) pour la palatalisation des voyelles et semi-voyelles (ụ, w..),

- et une barre qui marque la centralisation des voyelles fermées $(\dot{\mathfrak{i}}, \mathfrak{\sharp})$

Il faut de même ajouter que l'ASG est doté d'une police de caractère conçue et réalisée au Département d'Informatique du LUTO à partir du logiciel de traitement de texte SIGNUM 2 (Carpentier de Changy et Voltz 1990). 


\begin{tabular}{|c|c|c|c|c|c|c|c|c|}
\hline Minuscules & Majuscules & Mini & Maj & Mini & $M a j$ & \multicolumn{3}{|c|}{ Signes et dénominations } \\
\hline a & A & \multirow{16}{*}{$\begin{array}{l}\mathrm{b} \\
\mathrm{c} \\
\mathrm{d} \\
\partial \\
\mathrm{f} \\
\mathrm{g} \\
\mathrm{y} \\
\mathrm{h} \\
3 \\
\mathrm{j} \\
\mathrm{k} \\
? \\
\mathrm{i} \\
1 \\
\mathrm{~m}\end{array}$} & \multirow{16}{*}{$\begin{array}{l}\text { B } \\
\text { C } \\
\text { D } \\
\text { D } \\
\text { F } \\
\text { G } \\
\text { Y } \\
H \\
3 \\
J \\
K \\
? \\
L \\
M\end{array}$} & \multirow{16}{*}{$\begin{array}{l}\mathrm{n} \\
\mathrm{y} \\
\mathrm{p} \\
\mathrm{r} \\
\mathrm{r} \\
\mathrm{s} \\
\mathrm{f} \\
\mathrm{t} \\
\mathrm{v} \\
\beta \\
\mathrm{w} \\
\mathrm{w} \\
\mathrm{x} \\
\mathrm{y} \\
\mathrm{z}\end{array}$} & \multirow{16}{*}{$\begin{array}{l}\mathrm{N} \\
\mathrm{y} \\
\mathrm{P} \\
\mathrm{R} \\
\mathrm{R} \\
\mathrm{S} \\
\mathrm{S} \\
\mathrm{T} \\
\mathrm{V} \\
\beta \\
\mathrm{W} \\
\mathrm{W} \\
\mathrm{X} \\
\mathrm{Y} \\
\mathrm{Z}\end{array}$} & V̀ & \multicolumn{2}{|c|}{ infra-bas } \\
\hline ə & $\mathbf{B}$ & & & & & $\grave{v}$ & \multicolumn{2}{|c|}{ bas } \\
\hline e & $\mathrm{E}$ & & & & & $\overline{\mathrm{V}}$ & \multicolumn{2}{|c|}{ moyen } \\
\hline$\varepsilon$ & $\varepsilon$ & & & & & $\dot{v}$ & \multicolumn{2}{|c|}{ haut } \\
\hline i & I & & & & & $\hat{v}$ & \multirow{2}{*}{\multicolumn{2}{|c|}{ descendant }} \\
\hline 0 & 0 & & & & & V & & \\
\hline o & $\mathrm{O}$ & & & & & V̌ & \multicolumn{2}{|c|}{ montant } \\
\hline $\mathrm{u}$ & $\mathrm{U}$ & & & & & $\underline{\mathrm{v}}$ & \multicolumn{2}{|c|}{ très haut $(\mathrm{TH})$} \\
\hline un & U & & & & & $\dot{\mathrm{v}}$ & \multirow{2}{*}{\multicolumn{2}{|c|}{$\begin{array}{l}\text { haut abaissé } \\
\text { TH descendant }\end{array}$}} \\
\hline & & & & & & $\stackrel{\pi}{\mathrm{V}}$ & & \\
\hline Diacritiques & & & & & & \multicolumn{3}{|c|}{ Digraphes } \\
\hline \multicolumn{2}{|c|}{ Signes et Utilisations } & & & & & \multirow{5}{*}{$\begin{array}{l}\text { ny } \\
\text { ty } \\
\text { dy } \\
\text { kw } \\
\text { tw }\end{array}$} & \multirow{5}{*}{$\begin{array}{l}\text { mb } \\
\text { nd } \\
\text { yg } \\
\mathrm{kp} \\
\mathrm{gb}\end{array}$} & \multirow{5}{*}{$\begin{array}{l}\text { ts } \\
\text { dz } \\
\text { aa } \\
\text { oo } \\
\text { ee }\end{array}$} \\
\hline tild & our nasales & & & & & & & \\
\hline voy & très brève & & & & & & & \\
\hline pal & isation & & & & & & & \\
\hline cen & isation & & & & & & & \\
\hline
\end{tabular}

Tableau 3: Alphabet Scientifique des Langues du Gabon

La nécessité de permettre à tous les faits phoniques de toutes les langues d'être symbolisés et la volonté des descriptions phonologiques rigoureuses semblent avoir fait perdre à cet alphabet les critères de simplicité, de clarté et d'économie. L'ASG reprend également en compte des points particuliers (par exemple les signes c, j, y etc.) des usages officialisés par de nombreux pays africains.

L'étroit rapprochement de l'ASG aux alphabets phonétiques tels que l'API et l'alphabet Africa de l'IAI pose la question de sa caractéristique particulière. En effet, tandis que les alphabets API et IAI sont spécifiquement des alphabets pour des descriptions phonétiques et phonologiques - au sens proprement scientifique des termes, l'ASG est conçu par ses auteurs sans caractère spécifique. Hombert (1990a: 106-107), dans la présentation qu'il fait de l'ASG, est le premier à se demander implicitement s'il s'agit d'un alphabet phonétique ou d'un alphabet phonologique. Malheureusement l'auteur lui-même ne répond absolument pas à cette interrogation.

L'on retient que l'alphabet phonétique d'une langue est celui qui rend compte de la manière la plus fidèle possible, de la prononciation des locuteurs. L'inconvénient d'un tel système est qu'il reproduit totalement les signes de l'API et/ou de l'IAI, même si les inventeurs s'épargnent l'autre difficulté de créer des signes particuliers pour la notation des sons de la langue étudiée. L'alphabet phonétique a aussi 
l'inconvénient de contenir un grand nombre de signes dans le cas d'un système orthographique d'une langue.

L'alphabet phonologique quant à lui se limite à la prise en compte de la structure d'organisation des sons d'une langue. Il ne retient que la notation des sons pertinents de la langue, c'est-à-dire les phonèmes (au sens structuraliste et fonctionnaliste du terme). Il devra préalablement identifier les différentes variantes des sons d'une langue et ne retenir que l'une des variantes comme symbole pour toutes les variantes phoniques. Ce système implique l'utilisation d'un nombre réduit des signes mais nécessite irrémédiablement l'apprentissage de règles de lecture et d'écriture supplémentaire.

L'alphabet phonologique, comme indiqué en supra, reflète généralement le système et/ou la description phonologique de la langue et permet la confection d'un alphabet orthographique, c'est-à-dire un système simple et fonctionnel qui sert à l'écriture de la langue. L'alphabet orthographique est parfois éloigné des détails phonétiques et phonologiques d'une langue. C'est le cas de l'alphabet latin en usage dans bon nombre de langues européennes. Mais des digraphes souvent non inclus dans la liste alphabétique sont souvent utiles pour la symbolisation de quelques faits phoniques dans l'écriture ${ }^{10}$.

Je renvoie à l'article d'Afane Otsaga (dans ce volume) pour d'autres détails sur la distinction entre alphabet phonétique, alphabet phonologique et alphabet orthographique.

L'adjectif scientifique qui est associé à l'ASG semble dénoter qu'il a toutes les qualités et visées de l'API et l'IAI, c'est-à-dire la description linguistique. Mais l'assignation à chaque signe d'un correspondant majuscule laisse entrevoir que l'ASG a également des visées orthographiques. Autrement dit, l'ASG est à la fois un alphabet phonétique (par la prise en compte de nombreux détails phonétiques), un alphabet phonologique (puisqu'il est dit qu'il prend en compte l'essentiel des sons significatifs des langues) et un alphabet orthographique (pour l'écriture des langues).

Je pense que, comme l'alphabet africain de référence la non détermination de la nature de l'ASG (alphabet phonétique, alphabet phonologique ou alphabet orthographique) est l'une des raisons pour laquelle son application effective se fait toujours attendre.

Sur le plan orthographique, il n’a jamais été utilisé pour deux raisons bien évidentes.

10 A ce sujet l'on a par exemple, le digraphe th qui représente à la fois les sons [ə] et [ $\theta$ ] en anglais. Tout comme le son [s] qui a plusieurs symboles en français (s, ss, ç, c, et t) comme aussi en anglais (s et ss) et en espagnol ( $\mathrm{s}$ et $\mathrm{c})$. 
D'abord l'excès de scientificité de nombreux signes (par exemple les voyelles $\partial, \varepsilon$ et $\nu$, et les consonnes $\delta, \int$, $\gamma$ et $\beta$ entre autres) qui apparaissent barbares aux yeux des personnes profanes aux sciences du langage. Il faut noter que même au sein des universitaires et étudiants non initiés aux questions linguistiques, l'ASG est un alphabet qui rebute.

La seconde raison est tout simplement le fait que sa promulgation par les instances gouvernementales n'a jamais eu lieu. Le séminaire des experts qui a donné naissance à l'ASG a recommandé son adoption légale par le gouvernement et sa publication dans le journal officiel, mais cela n'a jamais été fait.

A mon avis, si l'ASG avait été spécifié comme alphabet orthographique, il aurait été nécessaire de réduire le nombre des signes spécifiques aux alphabets phonétiques (API et IAI) et le nombre des tons à transcrire. La simplification des diacritiques (au lieu d'être souscrits, ils seraient mieux superscripts comme cela se fait généralement partout ailleurs) et de digraphes aurait été également nécessaire.

Il faut aussi remarquer que l'ASG est relativement bâti sur les systèmes phonologiques de différentes langues du Gabon dont les experts suggéraient au cours de séminaires de propositions d'orthographe pour chaque langue décrite. Mais cela ne lui a pas empêché de trop considérer les variantes des phonèmes souvent également suggérés dans les orthographes de ces langues. Par exemple, dans l'esquisse phonologique que Blanchon (1990) fait du civili, il mentionne que le schwa ə est tout simplement une forme réduite du segment /a/ dans la langue. Il est cependant curieux de voir que l'auteur ne manque pas de prendre en compte à la fois le schwa et le segment /a/ dans sa proposition de l'alphabet orthographique du civili11, selon la simple raison que cette réduction est fréquente dans la langue. Pour le cas présent du civili il aurait été simple de choisir entre le /a/ et le schwa, et la maniabilité du premier porterait simplement le choix sur lui, ce qui donnerait un alphabet orthographique assez simple.

\section{L'ALPHABET D'AVRIL 1999}

Dix ans après la création de l'ASG, des experts pour l'essentiel gabonais réunis les 8 , 9 et 10 avril 1999 en session de concertation par le Ministère de l'Education Nationale et la commission nationale de l'UNESCO adoptèrent et mirent en place un système alphabétique pour l'orthographe des langues gabonaises.

11 Il faut reconnaître qu'au regard de la relation particulièrement complexe entre le schwa et la voyelle a en civili, il semble parfois nécessaire de pouvoir tenir compte des voyelles dans un alphabet orthographique du civili. L'exemple est ici présenté pour relever le fait que l'ASG prend en compte des phones en variation libre ou complémentaire dans beaucoup de langues gabonaises. 
Cet alphabet, reconnu comme le nouvel alphabet des langues gabonaises (Idiata 2002 : 54), et appelé par ses inventeurs sous le terme d'orthographe, fait suite aux recommandations des Etats Généraux de l'Education et de la Formation de 1983 qui insistèrent sur l'intégration des langues locales dans le système éducatif. Il tient également compte des conclusions et recommandations issues de la première table ronde sur les politiques linguistiques et l'enseignement des langues gabonaises de 1997 et celles des Etats Généraux du baccalauréat de 1998 qui relevèrent la nécessité de doter nos langues d'une orthographe, condition indispensable pour leur introduction dans les programmes scolaires.

S'appuyant sur les arguments de la simplicité et de la fonctionnalité, la Session de Concertation recommanda les graphèmes et les diacritiques, présentés dans le tableau 3 ci-dessous, pour l'écriture et la lecture des langues gabonaises.

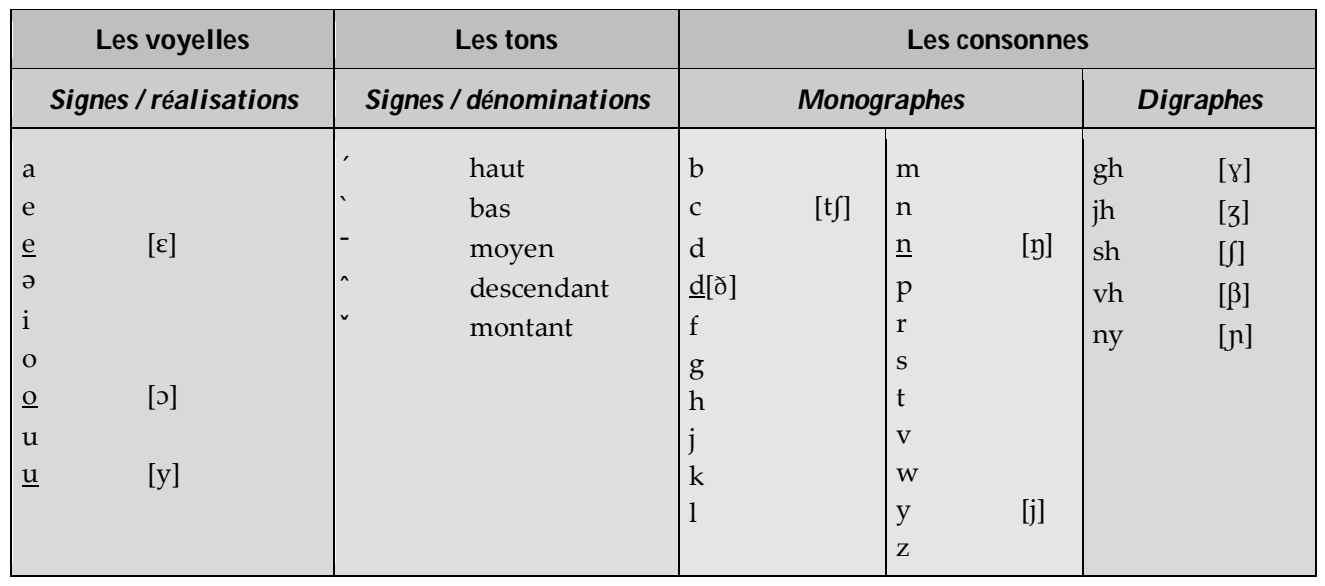

Tableau 4: $\quad$ Alphabet des langues gabonaises selon la session d'avril 1999

Cet alphabet qui contient 35 graphèmes dont $26^{12}$ consonnes et 9 voyelles constitue une avancée significative vers la simplification de l'écriture des langues gabonaises. L'allongement vocalique est représenté par le redoublement de la voyelle. Les mi-nasales et les complexes à glides s'écrivent à l'aide de deux ou plusieurs lettres mais comme la longueur vocalique, elles ne sont pas retenues comme des lettres à part entière de l'alphabet. Le diacritique affecté et identique à certaines lettres est souscrit $(\underline{\mathrm{d}}, \underline{\mathrm{e}}, \underline{\mathrm{n}}$, etc.). Ce système ne mentionne pas la notation des faits de nasalité vocalique ni ne recommande une forme majuscule des différents signes orthographiques.

Malgré le souci de fonctionnalité, cet alphabet retient la notation du schwa dans sa forme purement scientifique. De même, la lettre $\mathbf{j h}$ peut prêter à confusion puisque

12 Un décompte personnel à partir du rapport final de la Session indique 26 consonnes au lieu de 27 comme le mentionne Idiata (2002 : 54). 
le $\mathrm{j}$ indique déjà dans l'alphabet la plosive palatale [dz] et le h l'aspiration [h] (c'est selon l'API un son glottal fricatif). Ainsi la mise en commun des deux signes fait beaucoup plus penser à une plosive palatale aspirée. Cependant, les lettres gh et vh semblent cognitivement adéquats aux sons qu'ils représentent $(\gamma$ et $\beta$ ) à cause de la friction et la bilabialisation qui les distinguent respectivement de $\mathrm{g}$ et $\mathrm{v}$ (pour ce dernier c'est surtout sa coronalisation qui marque sa distinction avec la bilabiale $\beta$ ).

Par ailleurs, les inventeurs de cet alphabet avaient envisagé la notation de 5 tons tout en recommandant pour leur écriture orthographique la mise en place des commissions d'étude et d'harmonisation des différents systèmes linguistiques existants. Les experts recommandèrent également l'établissement des commissions pour l'application spécifique dans chaque langue de certains principes orthographiques examinées au cours de la Session de concertation. Toutes les commissions recommandées n'ont jusqu'alors pas vu le jour. De même, l'adoption par le Gouvernement et l'application des recommandations de la Session de concertation ainsi que du système d'orthographe proposé sont toujours attendues.

\section{L'ALPHABET ET L'ORTHOGRAPHE DE RAPIDOLANGUE}

Pour les besoins de l'expérimentation de sa méthode d'enseignement et d'apprentissage de langues gabonaises, la Fondation Raponda-Walker (cf. Hubert 1995 \& 1997) a élaboré également un système d'orthographe sur la base de la proposition d' Raponda-Walker. Les conditions et les modalités de confection de cet alphabet sont à lire dans ce volume dans l'article de Jacques Hubert. Les nombreux reproches faits à ce système par les linguistes gabonais - notamment sur la notation des tons - ont amené ses auteurs à réaménager leur système en tenant compte des signes recommandés dans l'orthographe des langues gabonaises issue de la Session de concertation évoquée plus haut.

Ainsi le système actuel de la méthode Rapidolangue, dont il faut lire les détails et la présentation dans l'article sus-cité de Jacques Hubert, est finalement une synthèse de la proposition de Raponda-Walker et de l'alphabet issu de la Session de concertation d'avril 1999. C'est un système qui maintient 9 voyelles, ë et e pour l'écriture respective du schwa et de la voyelle ouverte [ $\varepsilon$ ]. Les sons vocaliques [j] et [y] sont respectivement orthographiés $\underline{\mathrm{o}}$ et $\underline{\mathrm{u}}$ comme dans l'orthographe recommandée par la Session de concertation. La longueur vocalique est marquée par la voyelle redoublée, et la consonne $\mathbf{n}$ marque comme en français la nasalisation de la voyelle qui la précède.

Le nouvel alphabet de Rapidolangue est constitué de 24 consonnes. En dehors des consonnes simples et similaires à celles de l'alphabet latin, l'on note les digraphes dy (assurément pour le son interdental [ð]), dj (pour le son plosif palatal [dz]) et les 
mi-nasales $\mathrm{mb}, \mathrm{mp}$, nd et $\mathrm{ng}$ (ce dernier pour la nasale vélaire [y]). La nasale palatale [n] est alternativement notée ny ou gn sans tout autre forme de rigueur. La lettre $\mathrm{j}$ à défaut d'être un symbole pour [dz] est prononcé simplement comme en français [3]. Le signe y et le son qu'il peut représenter ne sont pas pris en compte dans l'alphabet, mais ils s'orthographient dans des mots comme omyene ou yipunu pour le y qui sert déjà dans certains digraphes. Les sons [J] et [t $\int$ ] comme en français ne sont pas envisagés dans l'alphabet, mais dans les mots où ils apparaissent, ils s'écrivent respectivement par sh et tsh comme en anglais : on a les mots comme shela (la chose en yinzebi), tshikë et tshivili (le banc et la langue vili en civili).

A la lecture des textes compris dans les manuels de Rapidolangue, l'on voit que les majuscules et la ponctuation sont également envisagées mais ne sont pas spécifiquement définis. La tendance est à les reproduire comme dans le système de l'orthographe latine.

Les tons sont désormais pris en compte dans l'écriture, mais dans la seule condition qu'ils soient indispensables dans le mot à écrire.

Une autre remarque importante qui se dégage de ce système d'orthographe est qu'il se rapproche énormément de l'alphabet latin. Ce qui lui donne un caractère très accessible pour des populations habituées à l'orthographe française et profanes aux sciences du langage. Ce rapprochement est assurément dû au fait que la méthode Rapidolangue a été initialement conçue pour les élèves du premier cycle des lycées et collèges qui ont pour la plupart le français comme première langue d'acquisition, et donc qu'ils parlent mieux que les langues locales et maîtrisent relativement le système d'écriture.

\section{PONCTUATION ET DIVISION DU MOT DANS LES LANGUES GABONAISES}

Aucun des alphabets ci-dessus présentés ne relève clairement sa position sur la ponctuation et la division du mot. Toutefois, l'observation des textes esquissés à partir de chaque système permet certaines suppositions. En ce qui concerne la ponctuation, il semble que tous les alphabets adoptent sans réserve le système de ponctuation des langues européennes. Ils semblent par contre divergents quant à la division du mot.

Les auteurs des différentes propositions alphabétiques n'ont peut-être pas perçu que le choix entre une écriture disjonctive par opposition à une écriture conjonctive est également fondamental. Raponda-Walker à la lecture de ses textes semble opter pour une écriture conjonctive, tandis que le Rapidolangue semble faire le choix d'une écriture disjonctive comme l'ASG au regard des extraits de contes présentés dans les ateliers du séminaire des experts. Mais dans Rapidolangue comme dans 
l'ASG les préfixes (nominaux et verbaux) et certains formatifs (particulièrement les formatifs de temps) sont écrits conjonctivement.

Pour l'écriture des langues gabonaises, il y a nécessité d'une harmonisation du système d'écriture conforme aux réalités morphosyntaxiques et phonologiques de ces langues. Je renvoie à Paul Achille Mavoungou (dans ce volume) pour des propositions en vue de la standardisation de l'écriture conjonctive ou disjonctive des langues du Gabon. J'ai également présenté plus haut que le choix du conjonctivisme ou du disjonctivisme était tout simplement une question de convention.

\section{ALPHABET ET CONSIDERATIONS TECHNOLOGIQUES}

De nombreuses langues africaines ont des systèmes phonologiques relativement complexes. C'est le cas de certaines langues du Gabon tels que le fang, le benga et bien d'autres langues de la zone B. C'est la raison pour laquelle dans la confection d'un alphabet orthographique, aux 26 lettres de l'alphabet latin bon nombre de symboles sont ajoutés. Cependant l'absence des symboles trop particuliers des claviers des machines à écrire (en particulier des ordinateurs) peut constituer une raison majeur pour laquelle un alphabet particulier de langue, en dépit d'être très bien accueilli par les professionnels des sciences linguistiques, peut échouer dans l'obtention d'une approbation générale.

Il faut également observer que l'alphabet est en lui-même une technologie (O'Connor 1996 : 787) qui nécessite un apprentissage aussi bien pour la lecture que pour l'écriture. Moins sont les difficultés de lecture et d'écriture plus accessibles et acceptables sont les éléments du système d'orthographe.

En ce qui concerne l'écriture au moyen des technologies modernes (ordinateurs et autres appareils électroniques), il nécessite que pour des alphabets tels que l'ASG, l'alphabet africain de référence, tout comme d'une certaine manière le nouvel alphabet des langues gabonaises issu de la Session de concertation, la création des logiciels et polices de caractères parfois particuliers pour pouvoir les adapter aux différents claviers. Autrement, leur usage aux niveaux technologiques élevés ne serait pas des plus faciles. Mais l'option des logiciels et polices particuliers implique de recherches interdisciplinaires (linguistique, sciences phonétiques et sciences informatiques) qui elles aussi exigent de moyens matériels et financiers assez importants que bon nombre de pays en voie de développement ne sont pas prêts de fournir.

Ainsi la création du système orthographique d'une langue peut faire appel à des efforts d'un niveau que l'on n'imagine pas de prime abord. 


\section{CONCLUSION}

Je viens de faire dans la présente contribution un bref historique du développement des alphabets des langues de manière générale. Je suis également revenu sur l'écriture des langues africaines telle que présentée par Touré (1990) tout en faisant certains rapprochements entre et avec les langues africaines ayant déjà ce que l'on peut appeler une tradition de l'écriture.

L'analyse de ces orthographes africaines est plus ou moins une source assez importante d'enrichissement dont peut s'inspirer un système d'orthographe standard des langues du Gabon. L'état des lieux sur l'écriture des langues gabonaises qui précède une analyse des alphabets existants montre le degré de retard du Gabon en ce qui concerne le développement concret des langues locales.

Les langues héritées de l'ère coloniale continuent de jouer un rôle important dans une Afrique post-coloniale en général, et au Gabon en particulier. La mise en place d'une écriture sur la base d'une orthographe inspirée de l'alphabet latin nécessite des conventions particulières qui soient accessibles et assimilables par des populations africaines qui, pour la plupart, sont habituées à l'alphabet latin par l'emploi des langues européennes.

En vue de faciliter la transition aux populations d'un système (celui des langues européennes) à un autre (celui d'une ou des langues africaines), les orthographes africaines ont un intérêt pratique (sur le plan technologique et sur le plan international) dans leur similarité aux orthographes des langues européennes. Ce précepte, cependant, interfère avec des principes analytiques que produiraient des orthographes scientifiquement motivées.

Il me convient de conclure avec Ndinga-Koumba-Binza \& Roux (2009: 100-104) qui, dans la prospection d'une proposition adéquate d'une orthographe des langues gabonaises, proposent de formuler préalablement un ensemble de principes en vue d'une forme standardisée. Celle-ci doit satisfaire non seulement à une expérimentation, mais aussi aux principes d'acceptabilité par la communauté qui aura à l'utiliser.

\section{REFERENCES}

Alihanga, M. 1990. Présentation du séminaire des experts pour un alphabet scientifique des langues du Gabon. Reoue Gabonaise des Sciences l'Homme 2 : 17-21. Libreville : LUTO/Université Omar Bongo.

Barber, CL. 1982. The story of language. Londres/Sydney: Pan Books. Edition revisée.

Blanchon, JA. 1990. Civili. Revue Gabonaise des Sciences l'Homme 2: 141-142. Libreville : LUTO/Université Omar Bongo. 
Carpentier de Changy, H. et Voltz, M. 1990. Alphabet scientifique des langues du Gabon: liste alphabétique. Revue Gabonaise des Sciences l'Homme 2 : 113115. Libreville: LUTO/Université Omar Bongo.

Coulmas, F. 1996. The Blackwell encyclopedia of writing systems. Oxford/Cambridge : Blackwell Publishers.

Gelb, I.J. 1963. A study of writing. Chicago : Chicago University Press. 2nd Edition.

Hombert, J-M. 1990a. Présentation de l'alphabet scientifique des langues du Gabon. Revue Gabonaise des Sciences l'Homme 2: 105-111. Libreville: LUTO/Université Omar Bongo.

Hombert, J-M. 1990b. Problèmes phonétiques et phonologiques rencontrés dans les langues du Gabon. Revue Gabonaise des Sciences l'Homme 2: 97-103. Libreville : LUTO/Université Omar Bongo.

Hubert, J. 1995. Rapidolangue: Méthode d'apprentissage des langues nationales. Volume 1 (fang, inzebi, lembaama, omyene, yipunu). Libreville: Editions Raponda-Walker.

Hubert, J. 1997. Rapidolangue: Méthode d'apprentissage des langues nationales. Volume 2 (ikota, ghetsogo). Libreville: Editions Raponda-Walker.

Idiata, F.D. 2002. Il était une fois les langues gabonaises. Libreville : Editions RapondaWalker.

Kidda, A. 1990. Historical background, with special reference to western Africa. R.R.K. Hartmann (ed.), Lexicography in Africa. Exeter: University of Exeter Press.

Kwenzi-Mikala, J.T. 1988. Quelques remarques sur la transcription des textes oraux en langues africaines PHOLIA 3: 207-211. Lyon: CRLS-Université Lumière Lyon 2.

Kwenzi-Mikala, J.T. 1990. Quel avenir pour les langues gabonaises? Revue Gabonaise des Sciences l'Homme 2: 121-124. Libreville: LUTO/Université Omar Bongo.

Mayer, R. 1990. Histoire de l'écriture des langues du Gabon. Revue Gabonaise des Sciences de l'Homme 2 : 65-91. Libreville: LUTO/Université Omar Bongo.

McLaren, J. 1936. A Xhosa grammar. Cape-Town: Longmans, Green and Co. Edition révisée par G.H. Welsh.

Ministère de l'Education Nationale. 1999. Rapport final de la Session de Concertation sur l'Orthographe des Langues Gabonaises. Libreville.

Mouguiama-Daouda, P. 1997. La substitution peut-elle expliquer l'unité linguistique du groupe myene ? Booga 1: 167-202. Libreville: Les Editions du Silence.

Ndinga-Koumba-Binza, H.S. \& J.C. Roux. 2009. On writing Gabonese languages. Batibo, H., Dikole, R., Lukusa, S., \& Nhlekisana, R., (Eds.) Language, Literature and Society. Proceedings of the First International Conference of the Department of African Languages and Literature, University of 
Botswana, Gaborone, Botswana, 26-28 June 2008. Gaborone: Associated Printers. p. 83-106.

O'Connor, M. 1996. The alphabet as a technology. P.T. Daniels \& W. Bright (Eds.). The world's writing systems. New York/Oxford : Oxford University Press.

Poulos, G. \& C.T. Msimang. 1998. A linguistic analysis of Zulu. Cape-Town : Via Afrika.

Raponda-Walker, A. 1998. Les langues du Gabon. Libreville: Editions RapondaWalker.

Republic of South Africa (Department of Bantu Education). 1972. Xhosa: Terminology and Orthography. Pretoria: The Government Printer.

Touré, A. 1990. "L'écriture des langues africaines: évolution et principes méthodologiques ». Revue Gabonaise des Sciences de l'Homme 2 : 55-63. Libreville : LUTO/Université Omar Bongo.

Van Wyk, E.B. 1995. Linguistic assumptions and lexicographic traditions in African languages. Lexikos 5 : 82-96. Stellenbosch : Buro van die WAT. 



\title{
ALPHABET ET ORTHOGRAPHE: \\ CRITERES, QUALITES, CONDITIONS ET VULGARISATION DANS LE CAS DU GABON
}

\author{
THIERRY AFANE OTSAGA \\ DEPARTEMENT DES SCIENCES DU LANGAGE \\ UNIVERSITE OMAR BONGO, LIBREVILLE \\ (afanotsaga@gmail.com)
}

\section{INTRODUCTION}

Alphabet et orthographe, voilà deux notions qui font jusque là l'objet d'un débat controversé dans le milieu de la recherche linguistique au Gabon. Ce débat, qui à mon sens n'est pas superfétatoire et ne saurait être confondu à une polémique ${ }^{1,}$ ne sera véritablement clos que lorsqu'un consensus aura été trouvé sur la question. Il faut dire que vu la situation actuelle des langues gabonaises, c'est plutôt l'absence d'un débat, sur la manière d'écrire ces langues, qui serait un fait étonnant et déplorable. En effet, personne ne peut nier l'évidence selon laquelle les langues gabonaises cherchent encore leurs marques au sein du concert linguistique moderne. Elles se débattent non seulement pour être préservées et ne pas devenir dans quelques années des langues mortes, mais aussi pour être utilisées de façon active dans tous les domaines de la vie moderne où leurs locuteurs exercent leurs activités. Pour que cet objectif soit atteint, les langues gabonaises se doivent nécessairement de passer par le même processus que celui emprunté par les langues qualifiées aujourd'hui de modernes et d'internationales (anglais, français, espagnol, etc.).

A mon avis, ce processus commence par l'adoption d'un mode ou système d'écriture unique reconnu et utilisé par tout le monde, c'est-à-dire un alphabet standard sans lequel toute tentative de développement des langues gabonaises

1 La différence entre débat et polémique est importante à faire dans le présent contexte. Le débat est un examen et une discussion au sujet d'une question ou d'un problème par des personnes d'avis différents. Sa finalité est de trouver un consensus ou une solution au problème qui se pose. Quant à la polémique, elle est beaucoup plus du domaine de la dispute. Chaque pôle ou camp n'a d'autre objectif que d'essayer de montrer que la raison est de son côté. 
resterait vaine. Le débat actuel sur le développement des langues gabonaises ne peut donc éviter le crucial problème de l'alphabet. Bien que d'aucuns estiment que les systèmes d'écriture qui existent actuellement sont en mesure de reproduire avec plus ou moins d'objectivité les besoins énonciatifs et scripturaux des langues gabonaises, encore faut-il tomber d'accord sur l'utilisation d'un système unique parmi ceux qui existent.

Mon objectif à travers cet article est donc d'apporter ma pierre à l'édification des langues gabonaises, particulièrement en ce qui concerne leur écriture. Il s'agit plus précisément de faire le tour de la question des systèmes alphabétiques. L'accent sera particulièrement mis sur les critères, les qualités, les conditions et la vulgarisation d'un alphabet. Ensuite quelques propositions seront faites en vue d'aider à avancer dans le processus de développement des langues gabonaises.

\section{APERÇU HISTORIQUE*}

\subsection{PRELIMINAIRES}

L'histoire du monde a prouvé qu'il a souvent été judicieux pour les sociétés humaines de s'inspirer des exemples des autres pour résoudre les problèmes auxquelles elles sont confrontées, et le développement des langues n'échappe pas à ce fait. Pour réussir le processus de développement de ses langues, le Gabon ferait acte honorable en s'inspirant des exemples des pays qui sont plus avancés dans le domaine linguistique. Pour ce qui est de l'alphabet orthographique, puisque c'est de cela dont il est question ici, une telle démarche implique une rétrospective succincte de l'histoire du développement des alphabets dans les pays dont l'avancé dans ce domaine est avérée. Cette rétrospective nous permettra de mettre en évidence un certain nombre de suggestions qui pourraient aider au choix et à la fixation d'un système alphabétique unique pour les langues gabonaises.

\subsection{LES ORIGINES DE L'ALPHABET}

Les premiers systèmes d'écritures sont nés sous des formes diverses à des époques distinctes dans de nombreux endroits du globe (Mésopotamie, Egypte, Chine, Amérique Centrale, etc.). En revanche, il semble bien que la naissance de l'écriture alphabétique soit géographiquement localisée en terre de Canaan, vers le 2ième millénaire avant Jésus-Christ, dans une région qui correspond aujourd'hui au Proche-Orient (Liban, Israël, Syrie, Jordanie et Sinaï). C'est donc plutôt du côté du Levant qu'il faut chercher l'origine de l'alphabet. L'ancien alphabet sémitique est

* L'essentiel des informations présentées dans cet aperçu historique est tiré du site web http:/histoire.typographie.org/carateres. Lire la première contribution de Ndinga-Koumba-Binza (dans ce volume) pour des informations complémentaires sur les origines des alphabets. 
d'abord un emprunt à la civilisation égyptienne. Cette écriture pseudohiéroglyphique fonctionnait selon le principe de l'acrophonie : chaque pictogramme symbolisait le tout premier son du mot représenté. Ainsi le signe de la maison, baytu représentait la « lettre » 'B'. Dans la mesure où dans les langues sémitiques, tout mot commence par une consonne, l'alphabet pseudohiéroglyphique était consonantique.

Parallèlement, était inventé à Ugarit, sur la côte phénicienne, aux alentours du 14 ième siècle avant notre ère, une écriture alphabétique consonantique de 30 signes utilisant le système graphique cunéiforme en usage dans l'ancienne Akkadie. Le cunéiforme disparu, l'alphabet linéaire poursuivit son évolution. Avant la fin du 12 ième siècle avant J-C, l'alphabet classique de 22 lettres arrivait à maturité après un millénaire d'évolution depuis l'invention des hiéroglyphes. La graphie des lettres se stabilisait de même que le sens de la lecture qui se faisait désormais de droite à gauche. L'alphabet phénicien découpait la syllabe en unités simples, les consonnes, et négligeait les voyelles qui servaient à les prononcer. L'acquis décisif demeurait: l'utilisation d'un ensemble réduit de signes graphiques pour symboliser la langue articulée.

\subsection{LA NAISSANCE DES VOYELLES}

La langue grecque, qui appartient au groupe indo-européen comme le persan, le sanscrit et la plupart des langues européennes, offrait des particularités qui en rendaient la notation difficile par l'écriture alphabétique consonantique phénicienne. La difficulté inhérente à toute écriture syllabique est d'isoler la consonne, non suivie d'une voyelle. Or les groupes de deux ou trois consonnes sont monnaie courante en grec: un texte grec dont les voyelles ne sont pas notées est ainsi complètement inintelligible. Pragmatiques, les Grecs transformèrent l'alphabet phénicien en l'adaptant à leur langue. Dans un premier temps, ils affectèrent à certaines consonnes phéniciennes, des valeurs à peu près similaires dans leur langue.

Ainsi, le signe du samek phénicien fut affecté à la consonne grecque de prononciation voisine $d u$ ' $s$ '. Après de nombreuses modifications d'orientation, ce caractère se stabilisa sous la forme du sigma, ' $\mathbf{S}$ ', tandis que le têt fut affecté à la notation du son th sous la forme du ' $Q$ ' et que le qof, $\mathbf{q}$, servit à noter le $\mathbf{k}$ et reçut le nom de koppa (' $\mathbf{K}^{\prime}$ ). Le zain sémitique, servit à noter le son grec $\mathbf{d z}$ sous la forme ' $\mathbf{Z}$ '. Mais l'invention la plus significative des Grecs consistera à attribuer à certaines lettres phéniciennes, dont ils n'avaient pas l'usage, la valeur de voyelle. C'est ainsi que naquirent le alpha ('A'), l'epsilon (' $\left.\mathbf{E}^{\prime}\right)$, l'omicron (' $\left.\mathbf{O}^{\prime}\right)$ et l'upsilon (' $\left.\mathbf{Y}^{\prime}\right)$. Pour la sonorité ' $\mathbf{I}$ ', ils inventèrent ex nihilo une lettre, le iota. Cette «lumière des voyelles» pour reprendre l'expression d'Etiemble, c'est l'apport décisif que vont faire les Grecs à l'histoire de notre civilisation. Le problème pour les Grecs n'était pas seulement de trouver un emploi pour les lettres sémitiques qui ne correspondaient 
pas à des consonnes de leur langue mais également d'arriver à noter tous les sons de cette dernière. C'est ainsi que le son $\mathbf{p h}$, fut d'abord noté 'PH' avant de se stabiliser sous la forme ' $\mathbf{F}$ '. Le son $\mathbf{k h}$ fut attribué à l'ancien taw sémitique, $\mathbf{C}$, resté sans emploi en grec. Le groupe consonantique ps, fut d'abord noté 'PS', mais les Ioniens recoururent rapidement au signe ' $\mathbf{Y}$ ' pour le représenter.

Ainsi, progressivement, son par son, signe par signe, s'élabora l'alphabet grec avec des différences notables selon les régions, mais suivant toujours le même processus: celui de l'adaptation du vieil alphabet sémitique à la langue grecque. Ceci explique d'ailleurs que les Grecs aient dans l'ensemble hérité des Phéniciens à la fois l'ordre dans lequel sont rangées les lettres et les noms de ces lettres. L'alpha rappelle indubitablement l'aleph phénicien, le bêta, le beth phénicien, etc. Au début les mots étaient écrits sans séparation; plus tard on les sépara les uns des autres. Dans le même ordre d'idée, les accents apparurent progressivement dans l'alphabet grec. Les Grecs écrivirent également dans un premier temps en boustrophédon ${ }^{2}$. Le boustrophédon constitue peut-être l'intermédiaire entre le sens phénicien, de droite à gauche, que les Grecs adoptèrent dans un premier temps et le sens ionien de gauche à droite. L'année -403 marque un tournant décisif dans l'histoire de l'alphabet grec. En effet, sous l'archontat d'Euclide, Archinos fit adopter à Athènes une disposition stipulant que les textes des lois, consignés jusqu'alors dans l'alphabet local, seraient réédités dans l'alphabet ionien. Les autres villes grecques, suivirent progressivement cet exemple, reconnaissant officiellement la supériorité de cet alphabet.

\subsection{L'INFLUENCE DE L'ALPHABET GREC DANS LE RESTE DU MONDE}

L'alphabet grec inspira les civilisations voisines. C'est ainsi que les Etrusques dont la civilisation apparue dans l'actuelle Toscane au 7ième siècle avant J.-C. reprirent l'alphabet grec pour transcrire leur langue. Des rois étrusques régnèrent sur Rome jusqu'au 4ième siècle avant J.-C. date à laquelle les peuplades originaires du Latium les chassèrent. Ces Latins, les futurs Romains, empruntèrent l'alphabet étrusque pour transcrire leur langue. $C^{\prime}$ est ainsi que vers le $3^{\text {ième }}$ siècle avant J.-C., fut établi un alphabet de dix-neuf lettres, le ' $\mathbf{X}$ ', le ' $\mathbf{Y}^{\prime}$ et le ' $\mathbf{Z}$ ' ayant dû être réintroduits dans $\mathrm{l}^{\prime}$ alphabet ${ }^{3}$ vers le $1^{\mathrm{er}}$ siècle avant J.-C. à l'époque de Cicéron. C'est de cet alphabet, dit alphabet latin, dont vont s'inspirer la plupart des civilisations latines (dont la France) pour créer les différents systèmes qui leur servent aujourd'hui d'alphabet.

2 Le boustrophédon est un système dans lequel le sens de lecture progressait à l'horizontale, alternativement dans un sens et dans le sens opposé, à la manière des bœufs au labour, revenant sur leurs pas à la fin de chaque sillon.

3 Les Etrusques avaient renoncés à ces lettres qui ne correspondaient à aucun son dans leur langue. 


\section{AlPHABET OU ORTHOGRAPHE / ALPHABET ET ORTHOGRAPHE}

\subsection{DEFINITIONS}

Avant d'engager toute discussion au sujet de l'alphabet et de l'orthographe des langues gabonaises, il est important de faire une analyse succincte des deux termes et de voir s'il s'agit de deux aspects qui renvoient à une même réalité ou s'il s'agit simplement de deux notions complètement différentes. Pour rappel notons que le terme alphabet est issu de la combinaison de alpha et beta, qui sont les deux premières lettres de l'alphabet Grec (qui correspondraient aux lettres a et $b$ de l'alphabet français). Aujourd'hui ce terme est utilisé dans un grand nombre de langues pour désigner une même réalité que certains dictionnaires et encyclopédies peuvent nous permettre de mieux cerner.

Le Nouveau Petit Larousse (1971) décrit l'alphabet comme une liste de toutes les

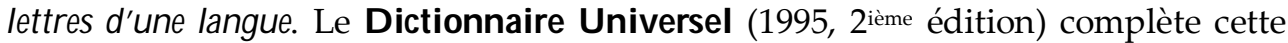
définition en décrivant l'alphabet comme l'ensemble des lettres servant à transcrire les sons d'une langue. Le Cambridge International Dictionary of English (1995) ne donne pas une définition très différente des deux premiers puisque l'alphabet y est décrit comme a set of letters arranged in a fixed order which is used for writing a language ${ }^{4}$. Microsoft Encarta Encyclopedia Standard (2001) est plus précis dans la définition de l'alphabet qu'il décrit comme set of written symbols, each representing a given sound or sounds, which can be variously combined to form all the words of a language. An alphabet attempts ideally to indicate each separate sound by a separate symbol ${ }^{5}$.

Comme nous pouvons le constater, toutes les définitions du terme alphabet, quel que soit la langue, se résument au fait qu'il s'agit d'un "ensemble de lettres ou de symboles servant à l'écriture d'une langue donnée". Le Tableau 1 nous présente quelques exemples d'alphabets de 4 langues non latines: l'Hébreu, le Cyrillique, l'Arabe et le Grec.

\footnotetext{
Une série de lettres arrangée dans un ordre fixe qui est utilisé pour écrire une langue.

Ensemble de symboles écrits, dont chacun représente un ou plusieurs sons donnés qui peuvent être variablement combinés pour former tous les mots d'une langue. Un alphabet essaye d'indiquer de manière précise chaque différent son par un symbole différent.
} 


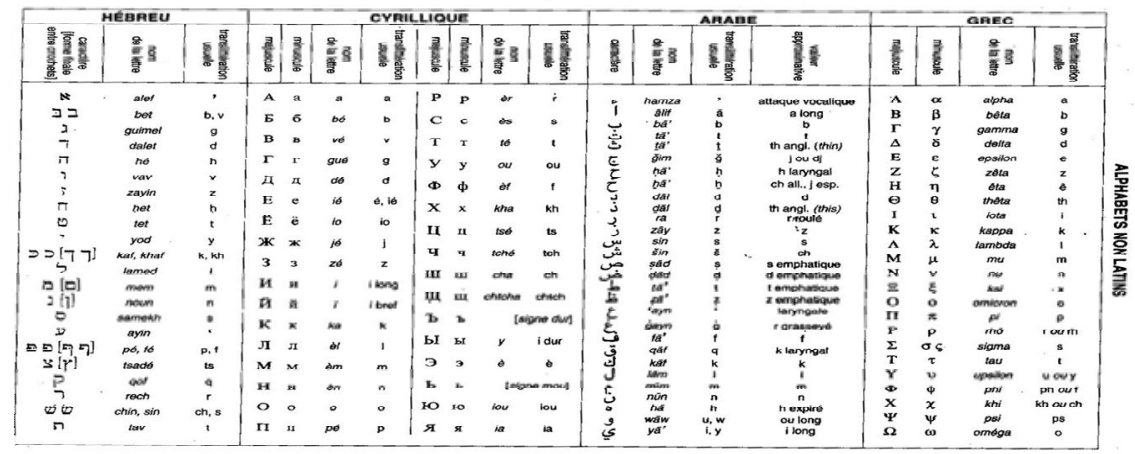

Tableau 1: Source tableau: Dictionnaire Universel (1995, 2ième édition)

Ce tableau nous donne trois types d'informations concernant les différents alphabets:

- Le caractère ou la typographie de chaque lettre, c'est-à-dire la manière dont chaque lettre est représentée ou transcrite dans la langue. Dans certains cas (Cyrillique et Grec) les transcriptions minuscules et majuscules sont données.

- Le nom de la lettre ou plus précisément la manière dont la lettre est prononcée.

- La translittération usuelle de chaque lettre, c'est-à-dire ce à quoi chaque lettre renvoi en alphabet latin.

- Dans le cas de l'arabe, des indications sont aussi données sur la valeur approximative de chaque lettre. Ces valeurs ont pour objectif de donner des précisions quant à la manière dont les lettres doivent être prononcées.

\subsection{ALPHABET PHONETIQUE ET ALPHABET ORTHOGRAPHIQUE}

Il est important de rappeler que le terme alphabet peut recouvrir deux aspects distincts de la transcription d'une langue: l'aspect orthographique (alphabet orthographique) et l'aspect phonétique (alphabet phonétique).On parle d'alphabet orthographique lorsque les lettres ou symboles servent à l'écriture des mots d'une langue. L'alphabet orthographique n'est donc pas à confondre avec l'orthographe, qui est, selon le Dictionnaire Universel, un ensemble des règles régissant l'écriture des mots d'une langue. En d'autres termes, l'orthographe est un système (réglementé) dans lequel les lettres de l'alphabet sont combinées pour former des mots (et par conséquent des phrases) d'une langue. L'orthographe peut donc être comparée à un système organisé dans lequel l'alphabet sert d'outil.

En français par exemple le terme empereur devrait s'écrire "enpereur" selon la manière dont il est prononcé. Mais, en tenant compte des règles orthographiques $\mathrm{du}$ français qui veut que $\mathbf{N}$ devant $\mathbf{P}$ devienne $\mathbf{M}$, c'est donc la lettre $\mathbf{M}$ qu'il faut utiliser pour écrire empereur. Les lettres utiliser pour écrire "empereur" viennent 
de l'alphabet du français, mais leur combinaison pour former ce mot s'est faite selon les règles qui régissent l'orthographe de cette langue. On parle donc d'alphabet orthographique pour désigner les lettres ou symboles qui sont utilisés pour former les mots d'une langue selon les règles orthographiques de cette dernière.

La situation n'est pas du tout la même en ce qui concerne l'alphabet phonétique. En effet, la phonétique a trait aux sons du langage. Elle permet de transcrire les sons de la parole exactement de la manière dont ils sont prononcés6. L'alphabet phonétique est donc constitué d'un ensemble de symboles, différents de ceux de l'alphabet orthographique, qui permettent de transcrire les sons de la parole quel que soit la langue utilisée. L'alphabet phonétique a pour objectif premier de permettre la transcription des sons d'une langue, même lorsqu'on n'en connaît ni l'alphabet, ni l'orthographe. C'est donc à juste titre que le Dictionnaire Universel considère l'alphabet phonétique comme moyen avec lequel on peut transcrire les sons de la plupart des langues. Cela explique donc la mise en place des symboles phonétiques internationaux tels que l'Alphabet Phonétique International (API) ou encore

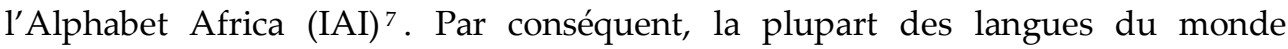
détiennent chacune un alphabet (orthographique), ainsi qu'un système orthographique particulier, beaucoup d'entre elles utilisent les mêmes symboles phonétiques $^{8}$.

Dans le cas du Gabon, une grande confusion persiste en ce qui concerne la distinction alphabet phonétique et alphabet orthographique. En effet, la question de l'écriture des langues gabonaises ne se pose pas en termes d'alphabet phonétique, puisqu'en dehors de l'API et de l'IAI, il existe aussi l'Alphabet Scientifique des Langues du Gabon (ASG) qui permet de rendre compte, de façon plus ou moins convenable, des sons dans les langues gabonaises. Le problème que rencontre les langues gabonaises est celui de l'alphabet orthographique. Il ne s'agit pas, en réalité, d'un débat sur la qualité des alphabets existants" mais sur la nécessité de choisir définitivement l'un d'entre eux et que seul ce dernier soit utilisé par tout le monde. C'est l'occasion ici de préciser qu'on ne peut faire référence à l'ASG lorsqu'il s'agit de transcrire les langues gabonaises

6 Cette définition de la phonétique est purement endogène. Elle ne tient pas compte des définitions usuelles de cette science qui est un domaine de la recherche linguistique. Ce domaine a pour objet la description physique des sons de la parole (Dictionnaire Universel, 1995).

7 l'IAI est né à cause du constat selon lequel l'API est plus adapté aux langues indo-européennes. Il ne permet pas de rendre fidèlement compte des particularités articulatoires et acoustiques de beaucoup de langues africaines.

8 L'anglais et le français par exemple sont deux langues différentes dans leurs systèmes phonétiques respectifs. Mais ils utilisent les mêmes symboles alphabétiques en ce qui concerne leur forme et de leur nombre.

$9 \quad$ Il est difficile de dire que tel alphabet est meilleur que tel autre puisque les critères utilisés par leurs auteurs étaient différents les uns des autres. Leur existence se justifie donc. 
orthographiquement. Les symboles retenus dans l'ASG sont trop proches des symboles phonétiques de l'API et de l'IAI. Il s'agit donc plus de symboles phonétiques qu'orthographiques comme nous pouvons le constater dans le Tableau 2 des correspondances ci-dessous.

\begin{tabular}{|c|c|c|c|}
\hline & $\begin{array}{l}\text { Alphabet Scientifique des } \\
\text { Langues du Gabon (ASG) }\end{array}$ & $\begin{array}{l}\text { Alphabet Phonétique } \\
\text { International (API) }\end{array}$ & Alphabet Africa (IAI) \\
\hline $\begin{array}{l}\text { C } \\
O \\
N \\
S \\
O \\
N \\
N \\
E \\
S\end{array}$ & $\begin{array}{l}\text { bc d f g g y r h } 3 \text { jk ky } ? 1 \\
\text { mnnyygrғs } \beta \text { w w..xyz }\end{array}$ & 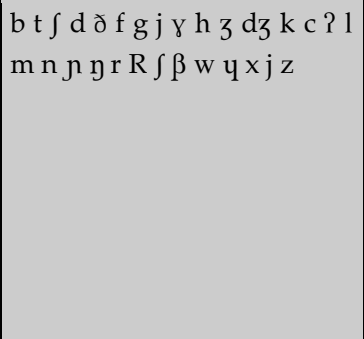 & 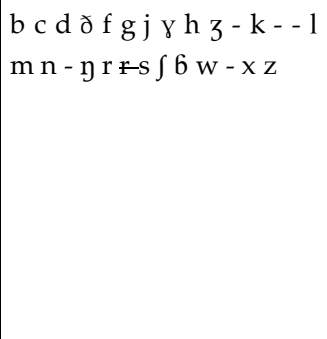 \\
\hline $\begin{array}{l}\text { V } \\
\text { O } \\
\text { Y } \\
\text { E } \\
\text { L } \\
\text { L } \\
\text { E }\end{array}$ & $\begin{array}{l}\mathrm{a} \\
\partial \\
\varepsilon \\
\mathrm{i} \\
0 \\
\mathrm{u} \\
\mathrm{u}\end{array}$ & $\begin{array}{l}\mathrm{a} \\
\partial \\
\varepsilon \\
\mathrm{i} \\
0 \\
\mathrm{u} \\
\mathrm{y}\end{array}$ & $\begin{array}{l}a \\
\partial \\
\varepsilon \\
i \\
0 \\
u \\
y\end{array}$ \\
\hline
\end{tabular}

Tableau 2: Notes: Seuls les symboles de l'API et de l'IAI ayant des correspondants dans l'ASG ont été présentés dans le tableau.

Il est vrai qu'un alphabet est avant tout une convention, par conséquent l'ASG peut être adoptée comme alphabet orthographique des langues gabonaises si toutes les parties décideuses (chercheurs et autorités politiques) s'accordent sur ce point. Mais personnellement j'estime que cela ne sera pas une très bonne idée, puisqu'une confusion risque de naître entre la transcription phonétique des langues gabonaises (rôle que remplit parfaitement l'ASG) et leur transcription orthographique. En d'autres termes, si l'ASG convient pour la transcription phonétique des langues gabonaises, il ne peut pas, en même temps, jouer le rôle $\mathrm{d}$ 'alphabet orthographique pour ces mêmes langues. Tous les autres systèmes d'écriture proches de l'API, ASG ou l'IAI (c'est-à-dire dont les symboles sont proches ou identiques aux symboles phonétiques) sont donc aussi automatiquement exclus comme potentiels systèmes d'écriture orthographique des langues gabonaises. 


\section{CRITERES ET QUALITES DE L'ALPHABET}

\subsection{LE FRANÇAIS ET LE CHOIX DE L'ALPHABET DES LANGUES GABONAISES}

Beaucoup de chercheurs estiment que la plupart des systèmes alphabétiques utilisés par les missionnaires pour la transcription des langues étaient calqués sur celui du français, et ils estiment que pour cela les symboles de ces alphabets ne rendent pas fidèlement compte des réalités des langues gabonaises. Cette vision est assez restreinte à mon avis, car avant de prendre une telle position, il convient de faire un certain nombre de remarques:

- Pour des raisons historiques, l'alphabet français est le système d'écriture avec lequel la population gabonaise alphabétisée est entrée en contact en premier. C'est aussi le système le mieux connu et le plus utilisé au Gabon, à cause du statut du français comme unique langue officielle dans ce pays. C'est donc le système d'écriture dont les populations gabonaises sont le plus accoutumées. Il est donc difficile de parler du problème d'alphabet au Gabon en faisant fi de cet aspect.

- La majorité des travaux pratiques (c'est-à-dire qui sont utilisables dans les milieux académiques ou religieux) qui existent aujourd'hui sur les langues ont été réalisés à base de ces alphabets dits calqués sur le français. Mais l'expérience montre que ces travaux ont eu un impact plus important sur la lecture et l'écriture des langues gabonaises, comparées à ceux réalisés à base des systèmes dits plus scientifiques ou plus en rapport avec les langues gabonaises. A titre illustratif, on peut aisément reconnaître que les Bibles et autres livres religieux, les dictionnaires, les lexiques, les glossaires, les syllabaires, etc. confectionnés dans les langues gabonaises avec un alphabet calqué sur le français ont permis à nos parents et grands-parents de pouvoir lire et écrire aisément en Fang, Yipunu, Omiénè, Yinzébi, etc.

- Comme nous l'avons vu dans l'aperçu historique, la plupart des alphabets qui existent aujourd'hui dans les langues du monde (surtout dans les langues Indoeuropéennes) sont en réalité le résultat des transformations séculaires de l'écriture égyptienne. Chaque pays, culture, civilisation ou peuple a adapté les symboles en fonction de ses besoins et de son contexte. Cela veut donc dire que ce n'est pas tant l'origine des symboles qui est importante, mais plutôt l'utilisation ou l'adaptation qu'on en fait.

- Enfin, nous ne devons pas oublier qu'un système alphabétique est améliorable et adaptable. Il n'est donc pas nécessaire qu'il soit unanimement satisfaisant dès sa mise en place. C'est au fur et à mesure qu'on l'utilise qu'on décèle ses lacunes. Celles-ci peuvent être quantitative (absence de symboles pour transcrire certains sons) ou qualitative (difficulté à rapprocher la transcription de la prononciation). 
Ces différentes remarques me permettent de faire les déductions suivantes:

- Il n'y a pas de critères particuliers pour le choix des symboles d'un alphabet. Celui-ci étant conventionnel, on peut prendre des représentions de tout genre (dessins, images, icônes, signes, graphiques, etc) pour en faire des symboles alphabétiques, à condition qu'à chaque son de la langue on fasse correspondre un symbole particulier. En plus de cela, il faut s'assurer que tous les sons pertinents de la langue sont bien représentés.

- Ce qui fait la force d'un système alphabétique, c'est son acceptation, son enseignement, son utilisation et surtout son adaptation. Toutes les langues, aujourd'hui à longue tradition écrite ont mis, des centaines, voire des milliers $\mathrm{d}$ 'années avant d'arriver aux versions finales actuelles de leurs systèmes alphabétiques. Il est donc utopique de penser qu'une quelconque réflexion, aussi poussée soit-elle, permettra de générer un alphabet orthographique automatiquement compatible avec l'ensemble des langues gabonaises. Toutes les propositions passées, actuelles ou futures des alphabets sont donc forcément susceptible de changements, de réaménagements, en termes plus clairs de réadaptations.

- Le choix ou la confection des alphabets, dans la plupart des langues dites développées, s'est toujours fait en tenant compte de ce qui existait déjà, même s'il s'agissait d'influence extérieure. L'ancien alphabet sémitique, par exemple, est d'abord un emprunt à la civilisation égyptienne, alors que les Grecs transformèrent l'alphabet phénicien en l'adaptant à leur langue. Choisir un alphabet orthographique basé sur le français, n'est donc pas un «crime de lèse acoustique et/ou phonatoire» ${ }^{10}$ vis à vis des langues gabonaises, à condition que celui-ci soit réadapté en tenant compte de ces langues. Bien au contraire, l'accoutumance des gabonais au système graphique du français peut faire en sorte que, si l'alphabet choisi est proche de celui du français, la population l'apprendra et le comprendra plus facilement.

\subsection{L'UTILISATION D'UN ALPHABET ORTHOGRAPHIQUE UNIQUE}

Il est important de noter que, s'il est scientifiquement possible d'obtenir un alphabet orthographique unique pour l'ensemble des langues gabonaises, il est par contre impossible d'obtenir un système orthographe unique pour l'ensemble de ces langues. En effet, n'oublions pas que l'alphabet orthographique est un ensemble de lettres ou de symboles utilisés pour l'écriture d'une langue, alors que le système orthographique est un ensemble de règles régissant le fonctionnement de chaque langue. Par conséquent, toutes les langues gabonaises ne fonctionnant pas de la

10 Beaucoup de gens pensent que les symboles du français ne traduisent pas de manière assez fiable les sons des langues gabonaises. 
même manière, elles ne peuvent pas avoir le même système orthographique ${ }^{11}$. Parler donc d'orthographe (en tant qu'ensemble de règles) des langues gabonaises est une hérésie. Par contre on peut parler d'une orthographe du Fang, du Yipunu, du Yinzébi, du Téké, etc. Il est à signaler que dans la plupart des littératures sur les langues gabonaises le terme orthographe est utilisé, non pas pour faire référence aux règles régissant l'écriture des mots de ces langues, mais pour parler des symboles utilisés pour la transcription des mots de ces langues. Vue dans cet esprit, le qualificatif "orthographe des langues gabonaises", pour parler de l'alphabet orthographique des langues gabonaises, peut donc avoir son sens. En ce qui me concerne et pour éviter toute confusion, dans cet article, j'utiliserai uniquement les attributs "alphabet orthographique" lorsque je parlerai des symboles orthographiques, "alphabet phonétique" pour parler des symboles phonétiques et "système orthographique" pour désigner les règles de fonctionnement de la langue.

Jusque là, plusieurs systèmes graphiques ont été utilisés pour la transcription des langues gabonaises. Cette situation n'est pas faite pour favoriser l'émergence de ces langues. Sans un système d'écriture unique aucune langue au monde n'a pu se développer. Pour aboutir à une avancée significative dans la résolution du problème de l'alphabet orthographique des langues gabonaises, il serait important qu'un consensus naisse enfin sur l'utilisation d'un système unique parmi les systèmes graphiques existants ${ }^{12}$. Parmi ces derniers, seuls trois d'entre eux semblent être à même de jouer le rôle assigné à un alphabet orthographique.

Le premier de ces systèmes est l'Alphabet des Idiomes Gabonais proposé par le premier scientifique Gabonais, Raponda-Walker et présenté dans Les langues du Gabon réédité en 1998 par la Fondation Raponda-Walker. Le chercheur propose pour la transcription des langues gabonaises 8 symboles vocaliques et 27 symboles consonantiques, recensés à partir de ses différents travaux (l'Alphabet des Idiomes Gabonais est disponible dans la première contribution de Ndinga-Koumba-Binza dans cet ouvrage).

La comparaison des différents symboles nous permet de confirmer que l'Alphabet des idiomes du Gabon s'inspire à la fois de l'API et de l'IAI (Ahmadou Touré, 1990:55). Ahmadou Touré (1990) ajoute que malgré les lacunes qu'il présente, cette orthographe (alphabet orthographique?) peut encore servir de base à l'élaboration d'un véritable alphabet scientifique des langues du Gabon. Idiata (2002:50) quant à

11 Le système orthographique de chaque langue gabonaise peut être obtenu à partir des différents travaux de description réalisés sur ces langues au département des sciences du langage de l’Université Omar Bongo. Il suffira pour cela de se baser sur les règles phonologiques, morphologiques et syntaxiques mises en évidence dans ces travaux.

12 A mon avis, il n'est pas utile de créer un nouvel alphabet. Ceux qui existent déjà peuvent faire l'affaire, il suffira de choisir l'un d'eux. 
lui estime que les propositions (les symboles) de Raponda-Walker s'appuient sur l'alphabet des langues latines.

Le second système qui semble répondre aux critères recherchés, c'est le Nouvel Alphabet des Langues gabonaises (ALG) proposé à Libreville en Avril 1999 au cours d'une session de concertation d'experts initiée par l'éducation nationale. Le choix des symboles de cet alphabet, qui comporte 9 voyelles et 26 consonnes, s'est fondé, selon les experts, sur leur simplicité et leur fonctionnalité (le Nouvel Alphabet des Langues du Gabon est disponible dans la première contribution de Ndinga Koumba-Binza dans cet ouvrage).

Le nouvel alphabet des langues gabonaises (ALG) a l'avantage d'être né d'une initiative du Ministère de l'Education Nationale et d'être issu d'une concertation d'experts. Il est donc le résultat d'une réflexion d'ensemble et mérite, par conséquent, une meilleure considération que celle qu'on lui a donnée jusque là. L'un des reproches qui pourrait être fait à cette proposition concerne le symbole $\boldsymbol{\theta}$ qui est identique à celui de l'alphabet phonétique. Il est important qu'une distinction soit faite entre les symboles orthographiques des langues gabonaises et leurs correspondants phonétiques pour que la prononciation soit à la portée de ceux qui désirent apprendre ces langues par le truchement de la phonétique. Il est reconnu que dans la plupart des langues gabonaises, le $\boldsymbol{\theta}$ est la réalisation de surface du a qui en est la réalisation sous-jacente. Pour éviter une confusion entre le $\boldsymbol{\theta}$ orthographique et celui phonétique dans l'écriture des langues gabonaises, le a pourrait être utilisé à la place du ə sans que cela change fondamentalement le sens $\mathrm{d}^{\prime}$ un terme ${ }^{13}$ à condition de présenter des règles de lecture pour les occurrences faisant apparaître le $\boldsymbol{\theta}$ au niveau phonétique. Mais dans le cas où la notation du $\boldsymbol{\partial}$ s'avèrerait indispensable, il pourrait par exemple être orthographiquement noté par un a souligné (â) qui permettra de le distinguer non seulement du $\boldsymbol{\theta}$, mais aussi du a habituel.

Le troisième et dernier système qui pourrait, a mon avis, remplir le rôle d'Alphabet orthographique des langues gabonaises est celui utilisé par la Fondation RapondaWalker pour la transcription des langues gabonaises dans les ouvrages Rapidolangue. Les symboles proposés dans cet alphabet sont d'après Idiata (2002:57) une synthèse de la proposition de Raponda-Walker et celle du nouvel alphabet des langues gabonaises (l'alphabet de la méthode Rapidolangue est disponible dans la contribution de Jacques Hubert dans cet ouvrage).

Du fait qu'ils soient une synthèse des deux premières propositions, les symboles de la méthode Rapidolangue ont l'avantage d'avoir tenu compte des insuffisances des premiers systèmes. Il a aussi l'avantage de s'écarter assez distinctement des symboles phonétiques.

13 Communication personnelle de Paul Achille Mavoungou. 
Des trois systèmes que je viens de présenter, seuls les deux derniers (à savoir le nouvel alphabet des langues Gabonaises et les symboles de la méthode Rapidolangue) ont vu leur mise en place certainement tenir compte des spécificités des langues gabonaises. Une fois encore la question ici n'est pas de plaider pour ou contre un système, mais de démontrer que le problème de l'écriture des langues gabonaises ne réside plus au niveau de la création d'un alphabet, cette étape ayant déjà été franchie grâce à la mise en place des systèmes (présentés en sus) aussi proches que possible des réalités ${ }^{14}$ des langues gabonaises. Au lieu de chercher a créer un nouveau système d'écriture, pourquoi ne pas s'appuyer sur ceux qui existent déjà, et les améliorer, si nécessaire. La tendance, au Gabon, à toujours vouloir remettre en cause les acquis du passé est un des freins au développement des langues gabonaises. Cette tradition du recommencement perpétuel ne nous mène à aucune avancée significative. L'idéal est de s'appuyer sur les propositions du passé et de les adapter en tenant compte des réalités nouvelles.

Beaucoup estiment que la résolution du problème de l'écriture des langues gabonaises se fera lorsque ces dernières auront été standardisées. Personnellement je ne suis pas tout à fait d'accord avec ce point de vue. A mon avis la standardisation d'une langue ne précède pas toujours celle de son alphabet orthographique, les deux peuvent (pour ne pas dire devrait) aller de paire.

\section{AlPhabet ET STANDARDisation}

\subsection{GENERALITES}

La standardisation d'une langue se fonde sur deux étapes principales:

- La création d'un modèle d'imitation.

- La promotion de ce modèle au détriment des modèles rivaux (Ray cité par James S. Mdee, 1999:120).

Le processus de standardisation d'une langue amène forcément à choisir une des formes ou variantes de la langue comme forme standard. C'est cette forme qui va donc servir de référence pour toute utilisation officielle et académique de celle-ci. Pour que le modèle choisi soit promu, particulièrement dans les écoles et les institutions académiques, il doit avoir une forme écrite. La forme standard d'une langue se fixe non pas oralement, mais à l'écrit, d'où la nécessité d'avoir un alphabet standard qui va permettre la vulgarisation du modèle de référence. En

14 Il n'y a pas de système orthographique parfait capable de rendre compte de toutes les spécificités d'une langue. Même dans le cas des langues Indo-européennes, les systèmes qui sont utilisés aujourd'hui pour leur écriture ont fait l'objet de nombreux débats et ne furent pas acceptés de prime abord lorsqu'ils furent proposés. 
faisant la promotion de la variante standard, on promeut aussi automatiquement la forme écrite sous laquelle elle est présentée.

L'illustration la plus évidente à ce sujet nous est donnée par le dictionnaire. En effet, à cause de l'autorité historique dont jouit cet ouvrage auprès des usagers (Afane Otsaga, 2002), la forme ou l'orthographe d'un terme serait difficilement remise en cause lorsqu'elle est présentée dans un dictionnaire. Car, pour la majorité des locuteurs natifs d'une langue donnée tout élément présenté dans un dictionnaire suffit à prouver sa véracité (Gouws, 2000a).

La standardisation des langues gabonaises va donc automatiquement entraîner celle de l'alphabet et de l'orthographe. Pour que cette standardisation connaisse une avancée significative et soit réussie, il est important que les chercheurs, les décideurs et la population s'entendent le plus rapidement possible, non seulement sur le choix des dialectes de référence, mais aussi sur la manière de les écrire.

\subsection{LE ROLE DES DECIDEURS DANS LE CHOIX D'UN ALPHABET}

Le fait que le Gabon, comparé aux autres pays africains, soit en retard par rapport au processus de développement de ses langues, peut, d'une certaine manière, être considéré comme un avantage. En effet, le Gabon pourrait (au mieux devrait) $s$ 'inspirer des exemples des autres pays pour gagner en temps et en argent ${ }^{15}$. Dans la plupart des pays (autant africains, européens qu'américains), le choix d'un dialecte ou d'un alphabet standard a souvent été la conséquence d'une influence intellectuelle ou politique.

En France par exemple une double évolution a caractérisé l'histoire du français au moyen âge. La première évolution s'est faite avec l'influence active de l'élite sociale et culturelle qui avait fait de leur usage du français la norme officielle au niveau administratif, diplomatique et littéraire. La deuxième évolution, toujours sous l'influence de cette même élite sociale et intellectuelle, s'est faite avec la marginalisation des autres dialectes du français qui furent confinés à n'être utilisés que dans les milieux populaires des provinces et dans les milieux strictement ruraux ${ }^{16 .}$

En Afrique, plusieurs pays tels que la Côté d'Ivoire (alphabet national de Côte d'Ivoire), le Nigeria (pan-nigérian), la Guinée, etc. ont élaboré des alphabets nationaux adaptés à la transcription de l'ensemble de leurs langues. L'adoption de ces alphabets a souvent été faite par le truchement de décisions politiques comme ce fut le cas en Guinée à travers l'ordonnance qui suit.

15 Les recherches linguistiques, pour des résultats efficients, nécessitent parfois des investissements financiers énormes. Pour les enquêtes de terrain par exemple le chercheur a besoin non seulement d'un matériel de pointe (enregistreurs, caméras, appareils photos, ordinateurs, logiciels, etc.) mais aussi de moyens de déplacement fiables (voitures tous terrains).

16 Ces informations sont tirées du Dictionnaire Universel, 1995, 2 ième édition. 


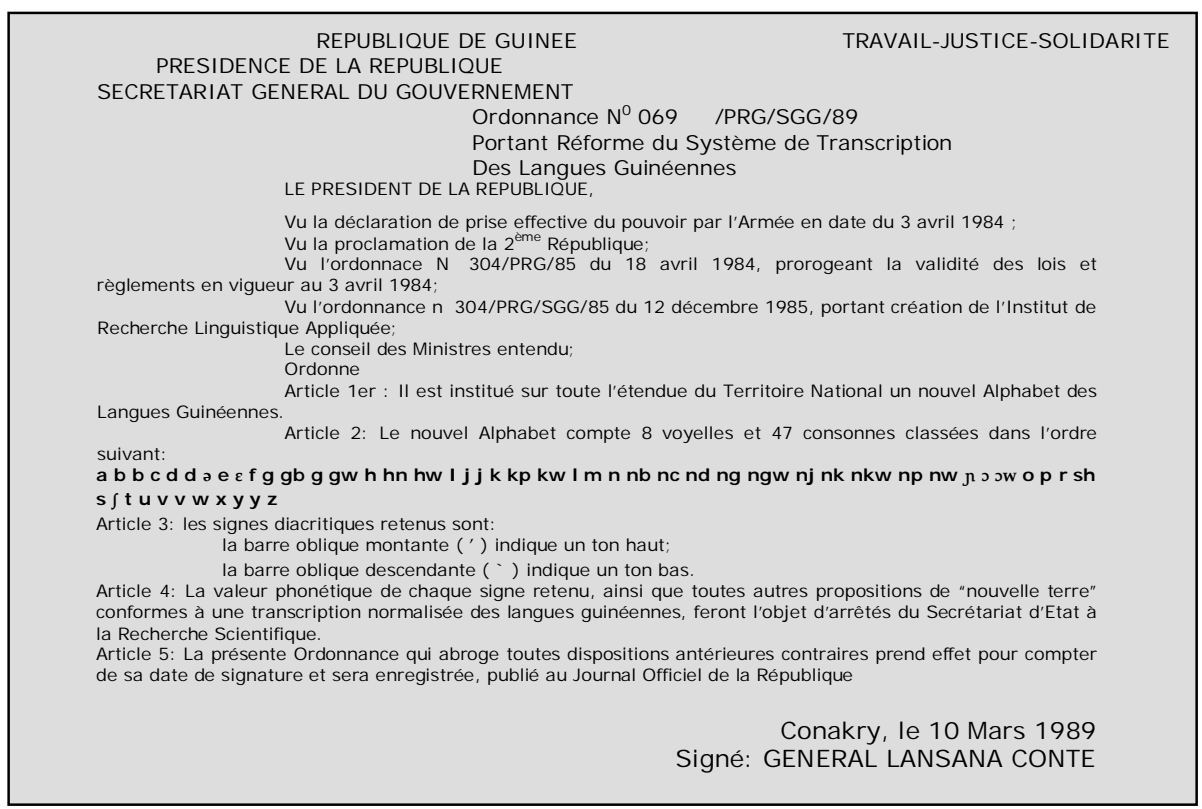

Extrait de la Revue Gabonaise des Sciences de l’Homme N 2, 1990.

Au Gabon, si on peut comprendre les hésitations du gouvernement à se prononcer sur le problème du choix des dialectes de référence ${ }^{17}$, il est par contre important, voire urgent, que celui-ci intervienne dans le choix d'un alphabet unique pour la transcription des langues gabonaises. Si l'alphabet choisi tient compte de l'ensemble des langues gabonaises, ainsi que leurs variantes, il pourrait donc être utilisé pour la transcription orthographique de n'importe quel dialecte de référence. Le choix de l'alphabet orthographique peut être fait avant celui des dialectes de référence.

La recherche d'un consensus entre experts est, bien sûr, importante pour le choix d'un alphabet. Mais puisque les uns et les autres n'arrivent pas à s'accorder sur la question, il revient donc au gouvernement de trancher au terme d'une concertation élargie (experts, populations, politiques). C'est à mon avis ce qui aurait dû être le cas au sortir de l'atelier d'Avril 1999 dont est issu le nouvel alphabet des langues gabonaises (ALG). Pour éviter les atermoiements actuels, le gouvernement, initiateur de cet atelier, aurait dû prendre un décret faisant de cet alphabet, le seul et unique système à utiliser pour la transcription des langues gabonaises ${ }^{18}$. Il est important de signaler que les cas où le gouvernement décide d'un l'alphabet, le choix n'est pas toujours unanimement accepté au départ, mais tout le monde finit par s'en accommoder. Il est également à indiquer que dans ce genre de cas, la

17 Ce choix implique beaucoup de facteurs sociaux, culturels et politiques dont il faut tenir compte.

18 Il n'est d'ailleurs pas trop tard pour que cela soit fait. 
fixation de l'orthographe est allée plus vite en comparaison avec les pays où elle s'est faite autrement.

Il est important de garder en esprit que le fait de choisir un alphabet ne résout pas automatiquement tous les problèmes de transcription dans une langue. Il n'y a pas de système parfait. Cela veut dire que celui qui est choisi doit être susceptible d'amélioration pour qu'il s'adapte aux nouvelles donnes. Il est par exemple possible qu'on se rende compte plus tard que l'alphabet choisi ne rend pas convenablement compte de toutes les spécificités des langues gabonaises ou que les sons de certaines langues ne sont pas transcriptibles avec ce système. Pour résoudre ces difficultés, l'alphabet choisi peut faire l'objet d'amélioration et $\mathrm{d}^{\prime}$ adaptation ${ }^{19}$. Mais pour savoir si le système choisi fonctionne ou non, il doit être expérimenté sur le terrain et seule cette expérience dictera la conduite à tenir pour l'avenir.

A mon avis, il n'est donc pas nécessaire d'être certain de la fiabilité d'un alphabet pour commencer à l'utiliser. C'est par son usage et son application qu'on découvrira ses faiblesses, qui sont susceptibles d'être améliorées. Le plus important étant de commencer. Il n'y a donc pas de raison d'hésiter de choisir et d'utiliser un alphabet sous prétexte qu'il ne rend pas entièrement et convenablement compte des spécificités de la langue. La recherche d'un alphabet idéal peut prendre des années, voire des décennies sans pour autant atteindre l'objectif escompté, surtout dans le cas du Gabon où l'inventaire, la classification et la description des langues locales restent une œuvre de longue haleine.

\section{UN MOT SUR L'ALPHABET ET LES TONS}

Il est difficile d'aborder le débat relatif à l'écriture des langues gabonaises sans aborder l'aspect lié à la notation des tons. En effet, le problème de l'écriture des langues gabonaises n'est pas seulement lié au choix du système alphabétique, mais aussi au dilemme de la notation ou non des suprasegments ${ }^{20}$.

Il faut noter que souvent deux points de vue au sujet de la notation s'opposent au sein de la communauté scientifique travaillant sur les langues gabonaises:

- Le premier point de vue est celui de ceux qui estiment que noter les tons lors de l'écriture des langues gabonaises est indispensable voire obligatoire puisque ces derniers jouent un rôle pertinent pour ne pas dire déterminant dans le fonctionnant de ces langues. Les partisans de ce point de vue argumentent

19 L'API n'est-il pas régulièrement actualisé pour faire face au caractère dynamique des langues du monde? La version actuelle de l'API a été dernièrement révisée en 2005.

20 Cet aspect est abordé plus en détails dans ce volume par L.S. Soami dans cet ouvrage. 
souvent que puisque les accents sont bien notés en français, il n'y aucune raison de ne pas noter les tons dans les langues gabonaises.

- Le deuxième point de vue est celui de ceux qui pensent que noter les tons est répulsif pour les lecteurs. De plus la bonne lecture des tons (leur prononciation) n'est accessible que pour les initiés de linguistique et pas au commun des lecteurs.

Quelques remarques s'imposent par rapport à ces deux points de vue. Il est un peu maladroit de comparer la notation des accents en français avec ceux des tons dans les langues gabonaises. Les deux types de suprasegments (accents et tons) ne fonctionnent pas de la même manière dans les langues en question. En français les accents font parties intégrantes des symboles alphabétiques (par exemple é, è, ê). Ces symboles sont donc toujours accompagnés de leurs éléments suprasegmentaux quel que soit leur situation ou leur contexte. Quant aux tons dans les langues gabonaises (comme dans toutes langues à tons), ils ne sont pas liés à un segment particulier. Ils peuvent donc apparaître au-dessus de tout type de segment syllabique en fonction de la hauteur mélodique du mot prononcé. Il est ainsi difficile d'associer un segment particulier à un suprasegment fixe. J'en déduis donc que la comparaison entre les accents en français et les tons dans les langues gabonaises n'est pas pertinente.

\section{VULGARISATION DE L'ALPHABET ORTHOGRAPHIQUE}

Le choix d'un alphabet orthographique pour la transcription des langues gabonaises ne suffira pas seul pour que le développement de ces langues devienne effectif. Il y a un certain nombre de mesures d'accompagnement nécessaires pour que le processus puisse véritablement aller à son terme et avoir les retombées escomptées. L'un des préalables à la fixation d'un alphabet c'est sa vulgarisation. La vulgarisation d'un système alphabétique n'est pas différente de ce qui se fait dans une société qui s'investit dans l'éducation populaire en vue de faire habituer aux populations une nouvelle initiative ayant la caution des plus hautes autorités du pays. Il va donc sans dire que les médias et les institutions académiques et politiques doivent être mis à contribution pour que l'objectif soit atteint. Ainsi des programmes télévisés en rapport avec l'écriture des langues gabonaises en utilisant l'alphabet choisi pourront régulièrement être diffusés ${ }^{21 .}$

21 Pour un tel objectif la télévision est le support médiatique le mieux indiqué parce qu'il a l'avantage d'être audiovisuel. En présentant l'alphabet ou en écrivant les langues gabonaises dans l'alphabet choisi à la télévision, cela fera en sorte que les populations s'y accoutument et commencent à l'intégrer dans leurs habitudes scripturelles. Il est plus facile de retenir ou de se rappeler quelque chose lorsqu'on le voit. 
Des émissions telles que le Polyglotte (RTG1) ou l'Académie des langues 22 (Radio Soleil) devraient être organisées à la télévision et non plus seulement à la radio. D'autres types d'émissions à l'instar de Question Pour un Champion (TV5, France) pourraient également être introduits dans les programmes de télévision. L'objectif est que les langues gabonaises soient utilisées non seulement comme supports discursifs, mais surtout que des "gros plans" soient régulièrement faits au cour des émissions pour montrer comment les termes utilisés doivent s'écrire. Lors d'une émission telle que le Polyglotte par exemple, les termes équivalents proposés par les participants devraient être présentés en gros plan sur l'écran pendant l'émission.

Les lieux d'acquisition du savoir (de la maternelle à l'université, en passant par les lycées, collèges et autres instituts d'alphabétisation), quant à eux, auront le rôle d'enseigner l'alphabet choisi aux apprenants. L'enseignement de l'alphabet des langues gabonaises devra donc être introduit dans les programmes scolaires. Pour que cela soit effectif, une décision gouvernementale obligeant tous les établissements scolaires (surtout maternels et primaires) à enseigner cet alphabet sera d'une importance capitale.

En ce qui concerne les chercheurs et les hommes de sciences, ils devraient utiliser l'alphabet choisi pour toute forme d'écriture et/ou de recherche impliquant les langues gabonaises. Les lexicographes et les linguistes, par exemple, devraient utiliser uniquement cet alphabet lors de la confection d'ouvrages en langues gabonaises. Les spécialistes des questions linguistiques et sociales (linguistes, lexicographes, pédagogues, sociologues, psychologues, etc.), auront aussi le rôle de veiller à ce que l'alphabet soit régulièrement adapté en fonction des nouvelles réalités (lorsque la description ${ }^{23} \mathrm{~d}^{\prime}$ une langue, par exemple, permet de constater que certains sons de ces langues n'ont pas de symboles représentatifs dans l'alphabet), grâce l'organisation régulière de séminaires et conférences impliquant toutes les parties concernées.

Les opérateurs économiques ne resteront pas en marge du processus de vulgarisation de l'alphabet des langues gabonaises. En effet, ces derniers pourront, à travers la publicité de leurs produits ou services, concevoir des spots publicitaires en langues gabonaises. Ces spots, qu'ils s'agissent d'affiches à travers les rues des villes du Gabon, ou de slogans à la télévision, devraient être faits avec pour

22 Le Polyglotte et $\mathrm{l}^{\prime}$ Académie de Langues étaient deux émissions de radio très populaires au Gabon. Dans celles-ci des termes étaient proposés en français par les présentateurs et les auditeursparticipants (par téléphone) avaient pour rôle de trouver et proposer les équivalents de ces termes français dans les langues gabonaises. Un même auditeur-participant a la possibilité de jouer dans plusieurs langues.

23 A ce sujet, l'apport des enseignants et étudiants du Département des Sciences du Langage (UOB) sera fort intéressant. Les descriptions linguistiques vont permettre la découverte des sons dont on n'a pas tenu compte dans l'alphabet choisi. 
support l'alphabet des langues gabonaises. La publicité se cristallise dans l'esprit des gens, dans le subconscient des individus, elle va aider à fixer l'alphabet dans les mémoires des populations.

Le Gabon n'étant pas en marge de la de communication moderne, l'alphabet des langues gabonaises pourrait, pourquoi pas, être publié sur Internet de sorte qu'il soit disponible pour un maximum d'usagers. De même les travaux réalisés dans les langues gabonaises, et ayant utilisés l'alphabet choisi, pourraient faire l'objet de publication sur le Web. Beaucoup d'internautes, qui sont aujourd'hui d'un nombre non négligeable au Gabon, ne manqueront certainement pas de chercher des informations sur les langues gabonaises. Ils vont ainsi s'habituer à lire ces travaux et s'accommoder au mode d'écriture utilisé.

\section{CONCLUSION}

L'écriture de langues gabonaises demeure un problème sur lequel toute personne soucieuse du développement de ces langues ne peut rester insensible. En effet, ce problème tient une place importante dans le retard que connaissent les langues gabonaises aujourd'hui.

A mon avis, les débats autour de ce problème ne sont pas inutiles tant que des avancées significatives n'auront pas été observées sur la question. Pour ce faire, le débat ne doit donc pas se résumer au constat du problème, mais il doit aboutir à des propositions susceptibles d'aider à apporter des solutions pratiques. De même, il est important de circonscrire aujourd'hui le niveau de la réflexion. Pour moi, il se situe en termes de choix d'un des systèmes alphabétiques existants, et non pas en termes de qualité du système à choisir.

Ma proposition se justifie par le fait que la plupart des systèmes alphabétiques existants dans les langues gabonaises sont à même de transcrire la majorité de ces langues, mais surtout à cause du fait qu'un système alphabétique, résultat de la décision des hommes, est susceptible de correction et d'amélioration. L'expérience des pays avancés dans ce domaine montre qu'un système alphabétique n'a jamais été parfait dès le départ, mais celui-ci acquiert ses lettres de noblesse avec la pratique (utilisation) et le temps. Le plus important est donc de commencer à l'utiliser et de le vulgariser, c'est cette étape que doivent franchir les langues gabonaises aujourd'hui. Si cet article (et les autres qui se trouvent dans cet ouvrage) peut servir à proposer des voies qui permettront de faire franchir cette nouvelle et importante étape aux langues gabonaises, alors il aura atteint son objectif. 


\section{REFERENCE}

Afane Otsaga, T. 2000. Esquisse phonologique et morphologique du Meka (langue bantoue de sigle A83). Mémoire de maîtrise. Libreville. Université Omar Bongo.

Afane Otsaga, T. 2001. Les dictionnaires bilingues dans les langues gabonaises: Approche méthodologique. J.D. Emejulu (ed.)in Eléments de Lexicographie Gabonaise, Tome I :137-159. New York: Jimacs-Hillman Publishers.

Anthonissen, C \& Gough, D. 1998. A Pragmatic or Purist Approach to Language Standard: Implications for outcomes based educational. Per Linguam 14(1). Stellenbosch: University of Stellenbsoch.

Dubois, J \& C. Dubois. 1971. Introduction à la lexicographie: Le dictionnaire. Paris. Librairie Larousse.

Emejulu, J.D. 2001. Lexicographie multilingue et multisectorielle au Gabon: Planification, Stratégies et Enjeux. J.D. Emejulu (ed.)in Eléments de Lexicographie Gabonaise, Tome I :38-57. New York: Jimacs-Hillman Publishers.

Gallardo, A. 1980. Dictionaries and the Standardization Process. L. Zgusta (ed) 1980. Theory and Method in Lexicography. Columbia: Hornbeam Press: 59-69.

Galley, S. 1964. Dictionnaires Fang-Français et Français-Fang, suivi d'une grammaire Fang. Neuchâtel : Henri Messeiller.

Gouws, R.H. 2000a. General Theory of Lexicography: An Introduction. M.Phil in Lexicography. Stellenbosch. University of Stellenbosch.

Gouws, R.H. 2000b. Equivalent Relation in Translation Dictionaries. M.Phil in Lexicography. Stellenbosch. University of Stellenbosch.

Gouws, R.H. 2001. Lexicographic Training: Approaches and Topics. J.D. Eléments de Lexicographie Gabonaise, Tome I: 58-94. New York: Jimacs-Hillman Publishers.

Idiata, D. F. 2002. Il était une fois les langues gabonaises. Editions Raponda Walker. Libreville.

Largeau, V. 1901. Encyclopédie Pahouine. Elément de grammaire et Dictionnaire Français-Pahouin. Paris : E. Leroux.

Largeau, V. 1901. Encyclopédie Pahouine. Eléments de grammaire et dictionnaire français-pahouin. Paris : E. Leroux.

Lejeune (R.P). 1872. Dictionnaire Français-Fang ou Pahouin, précédé de quelques principes grammaticaux sur cette même langue. Paris: A. Faivre et H. Teillard.

Mabika Mbokou, L. 2001. Le dictionnaire et le système éducatif, in Eléments de Lexicographie Gabonaise, Tome I : 206-222. New York: Jimacs-Hillman Publishers.

Marling (R.P.). 1872. Dictionnaire Fang-Français. New York.

Martrou, L. 1924. Lexique Fãn-Français. Paris Procure Générale (des Pères du St Esprit) / Abbeville : Imp. Paillard.

Matthews, P.H. 1997. Concise Dictionary of Linguistic. New York : Oxford University.

Mavoungou, P.A. 2001. La mondialisation et la lexicographie trilingue ou plurilingue au Gabon, in Eléments de Lexicographie Gabonaise, Tome I : 160-183. New York: JimacsHillman Publishers.

Nouveau Petit Larousse. 1971. Paris. Librairie Larousse.

Nyangone Assam, B. 2001. Le dictionnaire et l'enseignement des langues gabonaises. J.D. Emejulu (éd.) Eléments de Lexicographie Gabonaise, Tome I : 187-205. New York: Jimacs-Hillman Publishers. 
Prinsloo, D.J. 2001. The Compilation of Electronic Dictionaries for African Languages, in Lexikos 11. 139 -159. Stellenbosch. WAT. 



\title{
ORTHOGRAPHE, STANDARDISATION ET CONFECTION DES DICTIONNAIRES EN YILUMBU, YIPUNU ET CIVILI
}

\author{
PAUl ACHILle MAVOUNGOU \\ DePARTMENT OF AFriKAANS AND DUTCH, STELLENBOSCH UNIVERSITY \\ DePARTEMENT DES SCIENCES DU LANGAGE, UNIVERSITE OMAR BONGO \\ (moudika2@yahoo.fr)
}

\section{INTRODUCTION}

Sur la base des travaux de Guthrie (1953), le yilumbu (B44) et le yipunu (B43), langues bantu, sont classés en zone B40, plus précisément dans le groupe SiraPunu avec deux autres parlers: le ghisira (B41) et le yisangu (B42). Le civili, langue bantu également, est pour sa part classé en zone $\mathrm{H}$ avec le sigle H12a.

\section{DISTRIBUTION GEOGRAPHIQUE}

Le yilumbu est parlé au Gabon, en République du Congo et en République Démocratique du Congo. Au Gabon, le yilumbu est principalement parlé à Mayumba, Gamba et Setté Cama. Cette distribution géographique a donné lieu à l'existence de deux principaux dialectes: le yilümbu yi ghângu (la variante de la province de la Nyanga, en abrégée Ghâng.) et le yilŭmbu yí menaáne (la variante de la province de l'Ogooué-Maritime, en abrégée Men.). Bien que le niveau d'intelligibilité entre ces dialectes soit élevé, il y a un certain nombre de différences au niveau de la prononciation, de la grammaire et du vocabulaire.

Comme le yilumbu, le yipunu est également une langue dite transfrontalière en ce sens qu'il est parlé au Gabon, en République du Congo et en République Démocratique du Congo. Au Gabon, le yipunu est parlé principalement dans deux des neuf provinces que compte ce pays: la Ngounié et la Nyanga. Plus précisément à Mouila, Tchibanga, Ndéndé, Mabanda et Moabi (Kwenzi-Mikala, 1998a, 1998b et Nyangone Assam et Mavoungou, 2000). Il convient également de signaler la présence des Bapunu à Port-Gentil et à Lambaréné (On dit souvent qu'un tel est Mupunu de Lambaréné ou de Port-gentil, cf. Kwenzi-Mikala, 1980: 4). 
L'aire géographique du civili pour sa part s'étend aussi sur plusieurs pays à cause des frontières héritées de la colonisation.

Le civili est parlé au Gabon (particulièrement à Mayumba et Ndindi), en République du Congo (à Pointe Noire en particulier) et en Angola (dans l'enclave du Cabinda en particulier). Les lexicographes travaillant sur des langues ayant des variantes régionales sont généralement confrontés aux problèmes orthographiques et ils savent trop bien combien délicats sont ces problèmes.

\section{INTERET DU SUJET}

Comme mentionné ci-dessus, les problèmes liés à l'existence de variantes dialectales sont très complexes et la situation est pire lorsqu'une langue n'a pas encore été standardisée. Par rapport à ce point, le yilumbu, le yipunu et le civili restent à standardiser. Jusqu'à ce jour, le yilumbu ne dispose d'aucun ouvrage de référence ou dictionnaire. A l'opposé, le yipunu ne dispose à ce jour que de lexiques présentant des lemmes et leurs équivalents ou traductions.

Ces travaux produits par les missionnaires catholiques et protestants ou par les administrateurs coloniaux présentent difficilement des paraphrases du sens des lemmes traités (Nyangone Assam et Mavoungou, 2000; Mavoungou, 2001a; 2001b; 2002a et Mavoungou, Afane Otsaga et Mihindou, 2002c).

L'ouvrage de référence majeur disponible en civili est le Dictionnaire français-vili qui est une contribution du révérend-père Marichelle publié en 1902 sous les auspices de la Mission Catholique de Loango. Dans la majorité des langues du monde à grande distribution démographique, et disposant par conséquent de plusieurs variantes régionales, les techniciens de la planification linguistique sélectionnent généralement un dialecte susceptible de servir de variété standard.

Les critères traditionnellement proposés pour le choix dudit dialecte standard sont les suivants: l'importance numérique des locuteurs du dialecte en question, l'importance socio-économique dudit dialecte, la disponibilité de documents écrits dans le dialecte en question, etc., cf. Sadembouo (1980, cité dans Emejulu et NzangBié, 1999:39. Voir également Ndinga-Koumba-Binza dans ce volume.). Il est attesté que le choix du dialecte ou de la langue devant servir comme la norme ou forme standard est un problème très sensible dans les sociétés africaines en particulier. Par rapport au concept d'attitudes linguistiques, les gens tendent inévitablement à comparer les variétés d'une même langue entre elles.

Les locuteurs natifs vont généralement aimer, et c'est de bonne guerre, leur propre variété de la langue et rejeter ou critiquer les autres variétés. En ce qui concerne le yilumbu, ces idées préconçues se rencontrent souvent parmi certains locuteurs natifs du yilŭmbu yí menaáne qui considèrent souvent leur dialecte comme étant 
"pure" comparé au yilŭmbu yi ghângu. La "pureté" linguistique est tellement forte aux yeux desdits locuteurs natifs qu'ils se disent eux-mêmes Balumbu et parlent des locuteurs du yilŭmbu yi ghângu en termes de Bavili. Cet argument sur la "pureté" linguistique est relativement vrai si on garde à l'esprit les conclusions de l'étude comparative conduite par Blanchon (1989) sur le yilumbu, le civili et le yipunu. Blanchon (1984:33) écrit qu'approximativement 25\% du vocabulaire du yilumbu (la variante de la province de la Nyanga, spécialement celle parlée dans la région de Mayumba) est d'origine civili. Blanchon (1989: 32) mentionne également que le yilumbu, le yipunu et le civili ont environ $40 \%$ de vocabulaire commun.

Dans le même sens, il estime à 65\% le vocabulaire commun entre le yilumbu et le yipunu. Sur la base de données collectées jusqu'à ce jour, mon corpus montre que le yilŭmbu yí menaáne a plus d'affinité avec le yipunu. Par contre, dans le yilŭmbu yi ghângu les affinités linguistiques sont le plus élevées avec le civili. Il va sans dire que les décideurs, les techniciens chargés des questions de planification linguistique et les lexicographes devraient se départir de toutes ses affirmations basées sur le critère controversé de "pureté". Blanchon lui-même met en garde les chercheurs travaillant sur le yilumbu, le yipunu et le civili contre des conclusions hâtives:

Mais ce serait une erreur d'en conclure que le yilumbu est équidistant des deux autres parlers [yipunu et civili]. La phonologie et la tonologie du civili sont très différentes de celles du yipunu/yilumbu, et d'autre part les trois parlers ont de toute façon environ $40 \%$ de vocabulaire commun (si l'on tient compte des correspondances phonologiques systématiques). Le yi-lumbu est donc en fait un parler du même groupe que le yipunu, mais au contact du civili il semble avoir emprunté à ce dernier environ $25 \%(65 \%-40 \%)$ de son vocabulaire. Ce chiffre est une moyenne car, comme le montre le conte que nous venons de présenter, la proportion d'emprunts présumés au civili peut s'élever, pour les verbes courants, jusqu'à plus de 40\% (Blanchon, 1984:32-33).

Par rapport au critère controversé de "pureté", le yipunu parlé à Moabi et à Moukaba est souvent considéré comme la forme prestigieuse ou standard de la langue ${ }^{1}$. Dans le même ordre d'idées, le civili de Pointe Noire (cívíli cí njinji) est souvent présenté comme la forme standard de la langue.

D'un point de vue purement linguistique, il n'y a aucun doute que le yilumbu, le yipunu et dans une certaine mesure le civili sont des variétés d'une même langue. D'un point de vue sociolinguistique, les Balumbu, les Bapunu et les Bavili, entre autres choses, partagent le même préréquis culturel. Par conséquent, certains

1 On dit souvent qu'un tel n'est pas un vrai Mupunu tant qu'il n'a pas encore fait apparaître dans son discours des mots tels que "túmba" (mais), "bwéndíri" (que), etc. 
Balumbu parlent le yipunu et le civili et vice-versa, et ils se marient fréquemment entre eux.

Par rapport au concept de changements linguistiques, j'ai implicitement montré que dans une certaine mesure, d'autres langues bantu (en particulier le civili et le yipunu), et les langues européennes (le portugais, le français et l'anglais en particulier) ont influencé le yilŭmbu yi ghângu (Mavoungou 2002b). J'ai également souligné le fait que les locuteurs du yilŭmbu yí menaáne ont emprunté un nombre significatif de mots d'origine française et myene. Par rapport à ces faits, on peut arguer que les locuteurs à la fois des deux principaux dialectes ont eu recours à l'emprunt pour faire face à la demande croissante dans les domaines des sciences et technologies, le gouvernement, l'éducation, et le commerce.

Quand un dialecte est sélectionné pour servir de forme standard, il devient la forme pour l'écriture de cette langue ainsi que celle de l'administration et des médias. Tandis que, les autres dialectes sont confinés au domaine de la communication parlée ou informelle. Le choix du dialecte standard entre le yilŭmbu yi ghângu et le yilŭmbu yí menaáne va de toute évidence favoriser ceux/celles qui utilisent déjà la variante choisie et défavoriser ceux/celles qui ne l'utilisent pas. Pour éviter tout antagonisme au niveau social, une solution de compromis est à préférer au choix d'une seule et unique variante. Drame (2000:235-236) a fait remarquer que le développement d'un système d'écriture pour l'isiXhosa par John Bennie en 1824 était basé sur deux dialectes, notamment: le ngqika et le gcaleka.

Le même principe est applicable au yilumbu si l'on tient compte du fait que ses deux principaux dialectes sont largement inter-compréhensibles malgré quelques variations susceptibles d'apparaître au niveau de leur lexique. Le même raisonnement est valable pour le yipunu et le civili ainsi que pour d'autres langues gabonaises ayant des variantes régionales ou dialectes. En outre, il est important de mentionner que, la standardisation d'une langue ou d'un dialecte est très souvent initiée au niveau gouvernemental (par les planificateurs ou techniciens chargés de mener des programmes de planification linguistique) et non au niveau des communautés linguistiques.

Emejulu $^{2}$ a fait remarqué qu'en éditant un manuel de lecture et un coffret de cassettes audio sur la méthode et l'enseignement du mpongwe (l'un des six dialectes de la langue omyene), AYILE (organisation non gouvernementale dont le principal objectif est la promotion de la langue omyene et de la culture qu'elle véhicule) a implicitement choisi le mpongwe comme la norme ou forme standard pour l'omyene (voir aussi Nzang-Bie, 2001:21). Cette prise de position implicite pourrait s'avérer problématique parce que ce sont en définitive les populations

2 Communication personnelle, juillet 2001. 
Ngwe-Myene (la mère-myene $\underline{\text { e }}$ ) qui devront décider si oui ou non elles veulent du mpongwe comme forme standard.

Par rapport à ce qui précède, il est opportun de clarifier que ce n'est pas l'objectif de cette contribution d'appliquer les critères mentionner plus haut afin de choisir le dialecte standard pour le yilumbu, le yipunu ou encore le civili. Certains des problèmes soulevés ici découlent de la reconnaissance du fait que les dictionnaires jouent un rôle prépondérant dans la standardisation des langues. Cet aspect important est mieux résumé dans les propos de Mini (1995):

L'idée qu'a le public en général du dictionnaire comme source "exacte" du langage et par conséquent comme facteur significatif dans la standardisation du langage, le fait que le dictionnaire ait une influence sur l'usage du langage par les locuteurs...ainsi que le fait les lexicographes du isixhosa perçoivent leur travail comme une préservation de la langue isixhosa, placent une responsabilité lourde sur les lexicographes en ce sens qu'ils doivent s'assurer de maintenir un standard très élevé en termes de précision dans tous les aspects de leur travail (Mini, 1995:52, traduit par l'auteur).

C'est là un point très important et les lexicographes gagneraient à prendre en compte cette observation de Mini. Comme partie intégrante du processus lexicographique secondaire et intégral (Wiegand, 1998), un lexicographe gagnerait à s'assurer que son plan du dictionnaire n'est pas seulement une description sémantique d'une langue donnée mais aussi un guide pour d'autres aspects de l'étude du langage, les problèmes orthographiques en particulier. Après tout, le dictionnaire n'est-il pas le livre le plus influent à propos du langage [Ilson (1985:2) utilise le terme successful]?

Résumons-nous, la présente discussion se garde d'être la formulation d'un modèle théorique pour un dictionnaire standard $\mathrm{du}$ yilumbu, du yipunu ou encore $\mathrm{du}$ civili3 ${ }^{3}$ Il est largement attesté que la confection d'un dictionnaire standard peut jouer un rôle important dans le processus de standardisation d'une langue particulière. Selon Gouws (2001a), les dictionnaires standards doivent adhérer à un certain nombre de critères:

- Les dictionnaires standards peuvent être considérés comme des produits résultant d'un environnement lexicographique bien établi. Ces dictionnaires sont les instruments lexicographiques monolingues les plus utilisés et ils présentent un éventail large de lemmes et de catégories microstructurelles.

- Les dictionnaires standards sont généralement des produits à volume unique dominés par une approche normative. Bien qu'incluant un certain nombre de termes à fréquence d'usage élevée provenant des formes non standard, la

3 Afane Otsaga (2004) a formulé un modèle théorique pour la compilation d'un dictionnaire standard du fang à l’Université de Stellenbosch en Afrique du Sud. 
macrostructure des dictionnaires standards représente la variété standard de la langue traitée. Les items provenant des formes non standards seront marqués comme tel à l'aide d'étiquettes lexicographiques indiquant leur statut stylistique, chronolitique, régional ou encore au moyen d'autres déviations par rapport à la variété standard.

- Les dictionnaires standards incluent une sélection représentative des items macrostructuraux ainsi qu'un traitement extensif de ces items. Lesdits dictionnaires ont donc une densité élevée de données (Gouws, 2001a:76, traduit par l'auteur).

La présente contribution a uniquement pour but d'indiquer les différents moyens par lesquels, les dictionnaires peuvent être utilisés comme instrument dans la standardisation du yilumbu, yipunu et civili en particulier. Avant d'examiner les problèmes orthographiques en yilumbu, yipunu et civili, il est important de présenter des aspects essentiels liés aux efforts réalisés pour établir les conventions orthographiques pour les langues africaines en générale et pour les langues gabonaises en particulier (lire également Afane Otsaga et Ndinga-Koumba-Binza dans ce volume).

\section{EFFORTS PRODUITS POUR ETABLIR LES CONVENTIONS ORTHOGRAPHIQUES POUR LES LANGUES AFRICAINES}

C'est un fait bien attesté que comparé aux langues européennes par exemple, les langues africaines n'ont pas une riche tradition écrite. Autrefois, les langues et les cultures qu'elles véhiculent étaient exclusivement transmises oralement, excepté pour quelques langues comme le bamum (au Cameroun). Pour la majorité des langues africaines, la tradition écrite a débuté avec l'arrivée de l'évangélisation chrétienne et la colonisation sur le continent. La nécessité de traduire la Bible dans les langues à tradition orale ainsi que la production de matériaux didactiques dans lesdites langues pour les administrations coloniales qui ne souhaitaient plus dépendre des interprètes constituent les raisons souvent avancées pour transcrire ces textes oraux en langues africaines, et en langues gabonaises en particulier (Raponda-Walker, Busane, 1990 et Mavoungou, 2001a).

Même s'il faut accorder un crédit à ces travaux de la première heure, il n'en demeure pas moins qu'ils présentent quelques lacunes dans le domaine de la phonologie suprasegmentale (indications tonales) et de l'orthographe. En concevant des systèmes orthographiques pour les langues africaines, les chercheurs, les missionnaires et les administrateurs coloniaux ont généralement proposé un alphabet reflétant les réalités de leur langue maternelle. Les Anglais et les Américains - influencés par leur langue maternelle -ont produit des textes dans les langues africaines en fonction des conventions orthographiques de l'anglais et 
de l'américain respectivement, tandis que, les Français restaient "collés" aux traditions orthographiques $\mathrm{du}$ français.

Ce qui a abouti à des transcriptions approximatives à cause de la distance entre les systèmes phonétiques des langues africaines et ceux des langues européennes (Raponda-Walker, 1932:139-146; Kwenzi-Mikala, 1988:207 et Bendor-Samuel, 1996:689). Toutefois, il faut noter que ces travaux pionniers auraient gagné à être améliorés et par conséquent à servir de base aux travaux actuels. Dans la zone francophone, et au Gabon en particulier, la langue la plus connue pour ses problèmes orthographiques est le fang dont Alexandre (1961, cité par Kidda Awak 1990) dit qu'il a acquis:

...trois transcriptions [catholiques] et deux [protestants] pour environ un million de locuteurs, sans parler des différences morphologiques dues à des calques catholiques sur l'allemand, le français et l'espagnol, et protestants, sur l'américain (Kidda Awak 1990:11).

La citation ci-dessus montre clairement combien complexes sont les problèmes orthographiques. En effet, le fang souffre de la co-existence de deux principales formes standard (l'une dans la tradition établie par les missionnaires catholiques Français et une autre dans la tradition des missionnaires protestants Américains en particulier) ainsi que quelques variétés sous standard en provenance de l'espagnol et de l'allemand.

En jetant les bases de la standardisation des orthographes des langues gabonaises, en 1932, Raponda-Walker publie dans le Journal de la société des Africanistes l'article "Alphabet des idiomes gabonais" dans lequel il propose un ensemble de symboles susceptibles d'être utilisés pour la transcription des langues gabonaises. Par rapport à cet alphabet, il convient de noter qu'il repose sur le principe de biunivocité: un symbole pour un son. Cet alphabet est inspiré de l'ouvrage Essai de phonétique rédigé par Sacleux (1905). Il rejoint également dans ses grandes lignes The Practical Orthography of African Languages publié en 1928 par l'International African Institute [voir également, Ahmadou Touré (1990:55-63) et Kwenzi-Mikala (1998:219-220) pour des informations détaillées sur cet aspect].

Cinquante huit ans plus tard, l'Alphabet Scientifique des langues du Gabon (ASG, voir Carpentier de Changy et Voltz, 1990.) sera créé lors du Séminaire des Experts sur l'établissement d'un Alphabet Scientifique des langues du Gabon organisé par le LUTO (Laboratoire Universitaire de la tradition Orale, aujourd'hui Laboratoire Universitaire de la Tradition Orale et des Dynamiques Contemporaines, LUTODC) à Libreville. Ce séminaire s'est déroulé du 20 au 24 février 1989, et a vu la participation des délégations en provenance de trois pays: le Gabon, la Belgique (représentée par l'Université Libre de Bruxelles ainsi que par le Musée Royal de Tervuren), la France (représentée par l’Université Lumière Lyon 2) ainsi que par 
quelques experts en provenance de deux institutions internationales, le CICIBA (Centre International des Civilisations Bantu) et l'ACCT (aujourd'hui Agence Internationale de la Francophonie). Le séminaire avait pour objectif de concevoir un alphabet scientifique susceptible d'être utilisé pour la transcription des langues gabonaises.

La conception dudit alphabet a été suivie par une phase de mise en application au cours de laquelle, les différentes délégations travaillèrent en commissions afin d'évaluer l'utilisation pratique de l'alphabet proposé. L'Alphabet Scientifique des langues du Gabon (ASG) actualise également l'ensemble des symboles proposés par Raponda-Walker (1932). Il est basé à la fois sur les symboles de l'Alphabet International de Phonétique (API, révisé en 1993 et actualisé en 1996), et l'"Alphabet Africa" publié par l'International African Institute (IAI) très tôt après sa création en 1926. En sus, il met en application les conclusions de quelques conférences sur la standardisation des systèmes d'écriture pour les langues africaines organisées par l'UNESCO, à savoir: les conférences de Bamako (1966), Yaoundé (1970) et Niamey (1978). Enfin, les actes du colloque sont publiés dans la Revue Gabonaise des Sciences de l'Homme 2 (1990).

\section{LES SYSTEMES D'ECRITURE DU YILUMBU, YIPUNU ET CIVILI \& QUELQUES TENTATIVES VERS LEUR STANDARDISATION}

Cette section a pour objectif de rendre compte des systèmes d'écriture ainsi que des aspects liés à la standardisation. L'idée ici est de mettre en relief les variantes orthographiques répertoriées par un certain nombre d'auteurs, d'en faire un commentaire, et proposer de possibles améliorations.

\subsection{EFFORTS ENTREPRIS POUR ETABLIR LES CONVENTIONS D'ECRITURE POUR LE YILUMBU, YIPUNU ET CIVILI AINSI QUE LEUR EVALUATION CRITIQUE}

Comme cela apparaît clairement dans la section précédente, à l'exception du Bamum, très peu de langues africaines ont développé de systèmes d'écriture. Les explorateurs, les missionnaires et les administrateurs coloniaux ont développé des systèmes d'écriture en suivant les conventions des langues coloniales ou de colonisation.

S'agissant du yilumbu, des travaux précurseurs comme ceux de Garnier (1897, 1900, \& 1904) et Murard (1903) ont établi le système d'écriture de cette langue à partir des conventions orthographiques du français. Plus tard, Blanchon (1984) a rédigé un conte traditionnel en yilumbu en utilisant $l^{\prime \prime}$ Alphabet Africa". Emejulu et 
Pambo-Loueya (1990) ont proposé les symboles suivants pour le yilumbu: a, b, d, e, $\varepsilon$, ə, f, y, yw, l, k, kw, l, m, mb, n, nd, ndz, ng, $\mathrm{\eta}, \mathrm{o}, \mathrm{o}, \mathrm{p}, \mathrm{r}, \mathrm{s}, \mathrm{t}, \mathrm{ts}, \mathrm{u}, \beta, \mathrm{w}, \mathrm{y}$.

Comparé au yilumbu et au civili, le yipunu offre une littérature non négligeable. Mis à part de nombreuses productions religieuses, le premier travail qu'il convient de mentionner pour cette langue est la Grammaire pounoue et lexique pounoue-français par le révérend-père Bonneau (1956). Cet ouvrage pionnier est basé sur une série d'articles rédigés par l'auteur de 1940 à 1952 dans le Journal de la Société des Africanistes (JSA). La prochaine contribution qui mérite une mention spéciale est intitulée Éléments de description du Punu et a été éditée par Nsuka- Nkutsi (1980).

L'ouvrage en question est intéressant à plus d'un titre parce qu'il est à la fois un traité de grammaire et examine aussi le lexique du yipunu. En ce qui concerne le yipunu, les contributions de Kwenzi-Mikala (1980, 1989, 1990, 1998a, et 1998b, pour ne citer que celles-là) constituent un travail qui reste de premier plan. Tout chercheur intéressé par les langues africaines en générale et par le yipunu en particulier pourra consulter ces travaux avec profit (lire Nyangone Assam et Mavoungou pour des informations plus détaillées sur lesdites contributions). Il n'est pas sans intérêt de signaler que le yipunu est également l'une des neuf langues gabonaises enseignées, à titre expérimental, dans les lycées et collèges ${ }^{4}$ de Libreville grâce à la Méthode Rapidolangues initiée par la Fondation RapondaWalker (voir Jacques Hubert dans ce volume).

Comparé au yilumbu, le civili a une littérature non négligeable. Il s'agit surtout de textes religieux, tant bibliques que liturgiques. Sur un plan ethno-historique, de nombreux travaux existent. Citons par exemple, Le Testu (1918) et Meyer (1960). Cependant, l'ouvrage qui fait autorité depuis sa parution en 1991 est Autour du Loango par Merlet. Sur un plan purement linguistique, il convient de mentionner les contributions de Ndamba (1977), Blanchon (1984), Blanchon et Nsuka-Nkutsi (1984), Mabika Mbokou (1999) et Ndinga-Koumba-Binza (2000). Blanchon (1990) a proposé les symboles suivants pour le civili: a, b, c, d, e, f, j, k, l, m, mp, mb, n, nd, nt, nc, nj, nz, o, p, s, t, v, u, w, y, z. Enfin, le civili est l'une des neuf langues gabonaises enseignées, à titre expérimental, dans les lycées et collèges de Libreville avec comme support didactique les manuels d'apprentissage Rapidolangue de la Fondation Raponda-Walker.

Par rapport aux systèmes d'écriture, l'ouvrage de Le Testu (1918) contient une mine d'informations en ce sens qu'il répertorie les mots et expressions se rapportant à divers aspects culturels, les noms de lieux et des personnes. Un fait intéressant à mentionner concerne le titre de l'ouvrage en question. Bien que cette publication s'intitule Notes sur les Coutumes Bapounou dans la Circonscription de la

4 Institut Immaculée Conception, Collège Notre Dame de Quaben, Collège Bessieux, Collège Sainte Marie, Lycée d'Application Nelson Mandela. 
Nyanga, il s'agit en réalité d'un traité minutieux sur un certain nombre d'aspects culturels de la vie des Bapunu, Balumbu et Bavili vivant dans la province de la Nyanga. Le tableau suivant donne une illustration d'une variété d'items lexicaux représentant différents phénomènes culturels en yilumbu (abrégé Yil.), yipunu (abrégé Yip.) et civili (abrégé Civ.):

\begin{tabular}{|c|c|c|c|c|}
\hline Le Testu (1914) & $\begin{array}{l}\text { Graphie actuelle } \\
\text { des mêmes mots }\end{array}$ & Langues sources & $\begin{array}{l}\text { Transcriptions } \\
\text { phonétiques }\end{array}$ & Significations \\
\hline bifoula & bifula & Civ. et Yil. & [bìfúlə̀] $^{5}$ & esclave \\
\hline mwana & mwana & Yil., Yip. \& Civ. & [mwâ:nə̀] & enfant \\
\hline tchifoumba & cifuumba & Civ. & [cífù:mbə̀] & famille \\
\hline ibadou & yibandu & Yil., Yip. \& Civ. & [yìbâ:ndù] & clan \\
\hline boukali & bukali & Yil., Yip. \& Civ. & [bùkălì] & amende \\
\hline mwa foumou & mwa fumu & Yil., Yip. \& Civ. & [mwáfùmù] & personne noble \\
\hline ntekolo & $\mathrm{n}^{\prime}$ tekula $^{6}$ & Civ. & [ńtékùlə̀] & petit-fils/petite-fille \\
\hline foumou & fumu & Yil., Yip. \& Civ. & [fùmù] & chef; roi \\
\hline cama na combo & kama na kômbu & Yil., Yip. \& Civ. & [kámònàkó:mbù] & amende \\
\hline mboundou & mbundu & Yil., Yip. \& Civ. & [mbû:ndù] & $\begin{array}{c}\text { ordalie ou épreuve } \\
\text { du poison }\end{array}$ \\
\hline nganga kossi & nganga kosi & Yil., Yip. \& Civ. & [ngâ:ngə̀] & devin-guérisseur \\
\hline
\end{tabular}

Table 1: $\quad$ Liste des termes culturels tirés de Le Testu (1918)

Les items lexicaux dans le Tableau 1 ci-dessus présentent des lacunes à la fois sur le plan segmental et suprasegmental. Pour tous les travaux précurseurs, j'ai déjà émis une réserve par rapport à l'absence des tons dans la graphie des mots ${ }^{7}$. Un autre problème lié à celui qui vient juste d'être souligné est que la longueur vocalique n'est pas reconnue (par rapport à ce point, la convention la plus utilisée consiste au redoublement de la voyelle concernée).

5 Les symboles phonétiques utilisés dans cet article proviennent de l'Alphabet Scientifique des Langues du Gabon (ASG).

6 J'ai choisi de représenter orthographiquement la nasale syllabique [ń] par le symbole "n'" pour les raisons suivantes:(i) c'est une syllabe à part entière et (ii) elle se distingue de la nasale qui fait partie d'une consonne prénasale, cf. Ndinga-Koumba-Binza (communication personnelle).

$7 \quad$ D'un point de vue purement linguistique, il est important de donner des indications tonales aux lecteurs. Sans celles-ci, il est difficile de demander à ce dernier de reproduire correctement les sons du yilumbu, yipunu ou civili. 
Par rapport à ce qui précède, il est important de souligner que dans le Tableau 1 cidessus l'orthographe de la plupart des mots tirés de Le Testu (1918) diffère de la graphie actuelle des mêmes mots parce que l'auteur a utilisé les conventions orthographiques du français. D'un point de vue phonologique, le français ou qui est prononcé $[u]$ est utilisé pour représenter la voyelle yilumbu u comme cela apparaît dans la liste de Le Testu, par exemple foumou, boukali, bifoula, etc.

Un autre fait à mentionner est que la consonne du français $c$ est utilisée pour représenter la consonne yilumbu $\mathrm{k}$, par exemple cama et combo. Le système orthographique $\mathrm{du}$ français a des signes pour la gémination ou redoublement consonantique, cependant ce n'est pas le cas des langues gabonaises en particulier. Sous l'influence de sa langue maternelle, Le Testu a écrit le s du yilumbu, yipunu ou civili comme le français ss qui est prononcé [s], par exemple kossi (au lieu de kosi).

Des informations supplémentaires sur les conventions orthographiques apparaissent également dans les Tableaux 2, 3 et 4 ci-dessous. L'espace blanc signifie que la forme n'existe pas ou n'est pas connue de l'auteur. Le trait d'union (一) signifie que Le Testu n'a pas répertorié la forme en question:

\begin{tabular}{|c|c|c|c|c|}
\hline \multirow{2}{*}{$\begin{array}{l}\text { Noms des clans } \\
\text { dans la liste de Le } \\
\text { Testu (1918) }\end{array}$} & \multicolumn{2}{|c|}{$\begin{array}{l}\text { Noms des clans Bapunu } \\
\text { (Kwenzi-Mikala 1998: 3) }\end{array}$} & \multicolumn{2}{|c|}{$\begin{array}{l}\text { Noms des clans Balumbu (formes du singulier } \\
\text { tirées du corpus du yilumbu) }\end{array}$} \\
\hline & $\begin{array}{l}\text { Transcription } \\
\text { orthographiq } \\
\text { ue }\end{array}$ & $\begin{array}{l}\text { Transcription } \\
\text { phonétique }\end{array}$ & $\begin{array}{l}\text { Transcription } \\
\text { orthographique }\end{array}$ & Transcription phonétique \\
\hline Badoumbi & Badoumbi & [bàdû:mbì] & Mudumbi & [mùdû: mbì] \\
\hline Bassoumba & & & Musuumba & [músù:mbè] \\
\hline- & & & Muyẹma & [mùyémə̀] \\
\hline Bayengé & Badjengui & [bàjêngì] & $\begin{array}{l}\text { Muyengi } \\
\text { N'yenjii }\end{array}$ & $\begin{array}{l}\text { [mùyĕ:ngi] } \\
\text { [ńyĕ:ndzì] }\end{array}$ \\
\hline- & Dikanda & [dìkâ:ndò] & $\begin{array}{l}\text { Mwisi Dikanda } \\
\text { Mwisi Likanda }\end{array}$ & $\begin{array}{l}\text { [mwí:sìdikâ:ndè] } \\
\text { [mwísilikâ:ndè] }\end{array}$ \\
\hline- & Boumouéli & [búmwèlì] & $\begin{array}{l}\text { Mwisi Imondu } \\
\text { Mwisi Imondu }\end{array}$ & $\begin{array}{l}\text { [mwí:siímò:ndù] } \\
\text { [mwí:siímò:ndù] }\end{array}$ \\
\hline \multirow[t]{3}{*}{ Coucongo } & Minzoumba & [mínzû:mbə̀] & $\begin{array}{l}\text { Mwisi Kongu } \\
\text { Mwisi uKongu }\end{array}$ & $\begin{array}{l}\text { [mwí:sìkó:ngù] } \\
\text { [mwí:sìùsó:ngù] }\end{array}$ \\
\hline & Boudjiala & [bùjyălə̀] & & \\
\hline & $\begin{array}{l}\text { Dibamba- } \\
\text { Kadi }\end{array}$ & $\begin{array}{l}\text { [dìbâ:mbò- } \\
\text { kádì] }\end{array}$ & & \\
\hline
\end{tabular}




\begin{tabular}{|l|l|l|l|l|}
\hline & Didjiaba & [dijyăbə̀] & $\begin{array}{l}\text { Mwisi Diyaba } \\
\text { Mwisa Liyaba }\end{array}$ & $\begin{array}{l}\text { [mwísìdiyăbò] } \\
\text { [mwí:siliyăb̀̀] }\end{array}$ \\
\hline & Ndingui & [ndî:ngì] & Ndingi & [ndî:ygì] \\
\hline & & & Mughambu & [mùyâ:mbù] \\
\hline & & & Civacilwangu & [cívácilwâ:ngù] \\
\hline
\end{tabular}

\begin{tabular}{|c|c|c|c|c|c|}
\hline \multicolumn{2}{|c|}{$\begin{array}{l}\text { Noms des clans Balumbu/Bavili } \\
\text { (formes du pluriel, tirées du corpus } \\
\text { du yilumbu) }\end{array}$} & \multicolumn{2}{|c|}{$\begin{array}{l}\text { Variantes des clans } \\
\text { Balumbu/Bavili, (tirés du } \\
\text { corpus du yilumbu Corpus) }\end{array}$} & \multicolumn{2}{|c|}{$\begin{array}{c}\text { Noms des sous-clans des } \\
\text { Balumbu'et leurs sobriquets (tirés } \\
\text { du corpus du yilumbu) }\end{array}$} \\
\hline Trans. ortho. & Trans. Phon. & $\begin{array}{l}\text { Trans. } \\
\text { ortho. }\end{array}$ & Trans. phon. & $\begin{array}{l}\text { Trans. } \\
\text { Ortho. }\end{array}$ & Trans. phon. \\
\hline Badumbi & bàdû:mbì & & & & \\
\hline Basuumba & bàsù:mbà & & & $\begin{array}{l}\text { Ibebi } \\
\text { (Fumu), } \\
\text { Mughontsi, } \\
\text { Kaari et } \\
\text { Nzutsi }\end{array}$ & $\begin{array}{l}\text { [ỉbébì, } \\
\text { mùyô:ntsì, kă:rì, } \\
\text { nzútsì] }\end{array}$ \\
\hline Bayẹma & bàyémò & & & & \\
\hline $\begin{array}{l}\text { Bayengi } \\
\text { Bayenjii }\end{array}$ & $\begin{array}{l}\text { bàyĕ:ygì } \\
\text { bàyě:ndzì }\end{array}$ & & & $\begin{array}{l}\text { Kusu } \\
\text { (Fumu), Pili }\end{array}$ & [kúsù, pílì] \\
\hline $\begin{array}{l}\text { Bisi Dikanda } \\
\text { Basi Likanda }\end{array}$ & $\begin{array}{l}\text { bísìdìkâ:ndà } \\
\text { básilikâ:ndò }\end{array}$ & $\begin{array}{l}\text { Musi } \\
\text { Dikanda }\end{array}$ & $\begin{array}{l}\text { [músìdìkâ:nd } \\
\text { ə̀] }\end{array}$ & & \\
\hline $\begin{array}{l}\text { Bisi Imondu } \\
\text { Basi Imondu }\end{array}$ & $\begin{array}{l}\text { bísìimò:ndū } \\
\text { básiímò:ndù }\end{array}$ & $\begin{array}{l}\text { Musi } \\
\text { Imondu }\end{array}$ & $\begin{array}{l}\text { [músiímò:nd } \\
\text { ù] }\end{array}$ & $\begin{array}{l}\text { Ibaasa } \\
\text { (sobriquet) }\end{array}$ & [ỉbá:sə̀] \\
\hline \begin{tabular}{|l} 
Bisi Kongu \\
Basi uKongu
\end{tabular} & \begin{tabular}{|l} 
bísìkó:ygù \\
básiùkó:ngù
\end{tabular} & $\begin{array}{l}\text { Musi } \\
\text { Kongu }\end{array}$ & [músìkó:ngù] & & \\
\hline $\begin{array}{l}\text { Bisi Diyaba } \\
\text { Basi Liyaba }\end{array}$ & $\begin{array}{l}\text { bísìdìyăbò } \\
\text { básìlìyăbə̀ }\end{array}$ & $\begin{array}{l}\text { Musi } \\
\text { Diyaba }\end{array}$ & [músìdìyăbò] & & \\
\hline Ndingi & ndî:ngì & & & & \\
\hline Baghambu & bàyâ:mbù & & & & \\
\hline
\end{tabular}

8 Selon Kwenzi-Mikala (1998:3) Les noms des sous-tribus Bapunu sont: Djoundou ou Bagodjou [bàyójù], Simbou [š̌:mbù], Polou [pôlù], Ibassa [ìbâsò ] et [tăb̀̀]. 
\begin{tabular}{|l|l}
\hline Bivabilwangu & bívábilwâ:jgù
\end{tabular}

Tableaux 2 et 3: Noms des clans Balumbu, Bapunu et Bavili

Dans la liste ci-dessus comme dans le tableau précédant, la consonne du français $c$ est utilisée pour représenter la consonne yilumbu k, par exemple Coucongo (au lieu de Kukongu ou Mwisi uKongu) est la forme civili du yilumbu Mwisi Kongu. Le tableau suivant est une liste des noms de lieux dans Le Testu (1918) et KwenziMikala (1980). Le trait d'union (-) signifie que la forme n'a pas été répertoriée:

\begin{tabular}{|c|c|c|c|}
\hline $\begin{array}{l}\text { Noms de lieux } \\
\text { dans } \\
\text { Le Testu (1918) }\end{array}$ & $\begin{array}{l}\text { Noms de lieux } \\
\text { dans } \\
\text { Kwenzi-Mikala } \\
\text { (1980) }\end{array}$ & $\begin{array}{l}\text { Graphies actuelles des } \\
\text { mêmes mots dans le } \\
\text { corpus du yilumbu }\end{array}$ & Transcriptions phonétiques \\
\hline Loango $^{9}$ & Lwango & Lwangu & [lwângù] \\
\hline Loubinda & Doubinda & Dubiinda ou Lubiinda & [dùbǔrndè/ lùbǐ:ndè] \\
\hline Tchibanga & Tchibanga & Tshibanga & [cíbà:ygə̀] \\
\hline Dicoundou & - & Dikundu & [dikúndù] \\
\hline Penignioudou & - & Peninyundu & [pènìnû:ndù] \\
\hline - & Massanga & Masaanga & [másà:ngə̀] \\
\hline Penibatu & - & Penibatu & [pènìbátù] \\
\hline - & Moabi & Mwabi & [mwâ:bì] \\
\hline Moussitou & - & Musiitu & [mùsǐ:tù] \\
\hline - & Mouila & Mwila & [mwílì̀] \\
\hline
\end{tabular}

Tableau 4: Noms des lieux dans Le Testu (1918) et Kwenzi-Mikala (1980)

Comparé au tableau précédant, la donnée qu'il convient de mentionner dans le Tableau 3 est l'usage des digraphes $g n$ et $c h$. Avec le système orthographique du français comme point de référence, Le Testu a utilisé gn pour [n], par exemple Penignioudou (au lieu de Peninyundu) et ch pour [S], par exemple Tchibanga (au lieu de Tshibanga). Par rapport à ce qui précède, Il faut noter que dans le Rapport Final de la Session de Concertation sur l'Orthographe des Langues Gabonaises (1999), gh a été retenu pour [४], par exemple le mot signifiant "femme" est orthographiquement représenté comme mughetu en yilumbu et yipunu.

9 Loango, Loubinda, Doubinda, Tchibanga, Moussitou sont jusqu'à ce jour les dénominations officielles au niveau administratif. 
Loubinda et Doubinda sont tous les deux les représentations orthographiques d'un même toponyme en civili et yilumbu respectivement. Tous deux apparaissent en classe 14 avec le préfixe lu- en civili et du- en yilumbu et yipunu. Par conséquent, ce ne sont pas des variantes morphologiques.

Avec le système orthographique du français comme point de référence, les travaux des précurseurs distinguent difficilement certaines voyelles entre elles-mêmes si ces dernières sont phonologiquement distinctives, par exemple [e] et $[\varepsilon]$ sont toutes les deux représentées par $e$, et [o] et [o] sont toutes les deux représentées par 0 . Le Rapport Final de la Session de Concertation sur l'Orthographe des Langues Gabonaises (1999) s'est éloigné de cette approche en proposant l'usage de signes diacritiques. Dans ledit rapport, le e souligné et le o souligné sont utilisés pour transcrire $[\varepsilon]$ et [0] respectivement.

Comme partie intégrante de la planification de la macro- et microstructure d'un dictionnaire, un lexicographe devrait non seulement rendre compte des critères de sélection pour les nombreuses variantes de mots à travers les différents dialectes d'une langue particulière, mais également les nouvelles formes qui ont été incorporées au fonds lexical d'une langue comme résultat du contact de langues, principalement par le truchement de l'emprunt. Watch (1993) et Raponda-Walker (1933) contiennent des informations pertinentes dans ce sens comme cela apparaît dans les tableaux suivants. L'espace blanc signifie que la forme en question n'existe pas dans mon corpus:

\begin{tabular}{|l|l|l|}
\hline \multicolumn{1}{|c|}{$\begin{array}{c}\text { Liste deWatch } \\
(1993)\end{array}$} & \multicolumn{1}{|c|}{$\begin{array}{c}\text { Graphies actuelles dans le } \\
\text { corpus du yilumbu }\end{array}$} & \multicolumn{1}{c|}{ Transcriptions phonétiques } \\
\hline Santu & santu & [sâ:ntù] \\
\hline Krusu & krúúsu & \\
\hline Ekaristi & & [kómìnì] \\
\hline Kommunion & kominyi & \\
\hline Santa Kommunion & & \\
\hline Paradi & & [krétì] \\
\hline Kretien & kréti & [yé:sù, fùmù] \\
\hline Jezu & Yeesu, Fumu & [bátè:mò] \\
\hline Batema & bateema & \\
\hline Lekaristi & & \\
\hline Penitansi & & [nówè:là] \\
\hline Noela & Noweela & [ìngángò, ntsùbùnzâ:mbì] \\
\hline Egliza & Inganga, nzubu Nzâmbi &
\end{tabular}


Tableau 5: Termes religieux empruntés dans Watch (1993)

\begin{tabular}{|c|c|c|}
\hline Liste de Raponda-Walker (1933) & $\begin{array}{l}\text { Graphies actuelles des mêmes mots } \\
\text { dans le corpus du yilumbu }\end{array}$ & Prononciation \\
\hline kabala & kuvhaalu, kavhaala & [káßà:lù], [káßà:lè] \\
\hline salu & saalu & [sá:lù] \\
\hline mensa & meetsa & [mê:tsə̀] \\
\hline \multicolumn{3}{|l|}{ cadella } \\
\hline karesa & kárasa & [kárə̀sə̀] \\
\hline saku & saaku & [sá:kù] \\
\hline nsabaku & nzabaaku & [nzábà:kù/ ntsábà:kù] \\
\hline sabela & Sabala & [sábə̀là] \\
\hline lumingu & Dumiingu & [dùmǐ:ngù] \\
\hline
\end{tabular}

Tableau 6: Termes divers empruntés dans Raponda-Walker (1933)

Le fait à souligner dans les tableaux ci-dessus est que les conventions orthographiques des langues d'origine (le français pour la liste de Watch et le portugais pour la liste de Raponda-Walker) ont été retenues lors du processus de l'emprunt, par exemple la gémination (redoublement consonantique) apparaît, la quantité vocalique n'est pas reconnue et la notation du schwa est omise. La prise en compte des particularités phonétiques des langues gabonaises se serait traduite par la suppression des lettres en trop dans des termes empruntés comme Kommunion et Santa Kommunion. À cause de tout cela, des exemples tels que Jezu (au lieu de Yeesu) et Egliza (au lieu de inganga) — dans la liste de Watch semblent être plus des cas d'emprunts forcés que de termes résultant d'un véritable processus d'emprunt.

Par contre, dans la liste de Raponda-Walker un son distinctif correspond plus au moins à un seul symbole et réciproquement (principe de bi-univocité). Ceci est important parce qu'un alphabet est sensé refléter la prononciation des mots d'une langue. En dépit de l'absence de tons dans la graphie des mots, presque tous les termes empruntés apparaissant dans la liste de Raponda-Walker sont connus des Balumbu. Mis à part ces problèmes liés à la graphie des emprunts linguistiques, le choix entre une écriture conjonctive par opposition à une écriture disjonctive est également fondamental. Raponda-Walker a opté pour une écriture conjonctive, tandis que Watch a fait le choix d'une écriture disjonctive. Les données de la liste suivante sont extraites de Watch (1993): 


\begin{tabular}{|l|l|}
\hline Mots & Significations \\
\hline ma ngolo & La force \\
\hline ma sumu & Les péchés \\
\hline ba kallanga & Ils habitaient \\
\hline bi lumbu & Les jours \\
\hline Lu Mingu & dimanche \\
\hline Tchi lunzi & L'esprit \\
\hline
\end{tabular}

Tableau 7: Exemples d'écriture disjonctive dans Watch (1993)

Une question importante se pose ici: Où mettre les lettres majuscules? De manière générale pour honorer Dieu, tous les termes se rapportant à lui ou à quelque chose ayant un lien avec lui devraient être orthographiés avec l'initiale en majuscule et ce même dans le discours ordinaire. Par rapport à ce point, dans la liste de Watch Jezu (Jésus- Christ) est écrit avec un "J" majuscule.

Toutefois, l'entrée Lu Mingu (dimanche) est un exemple intéressant parce que commençant avec un "L" majuscule et ayant également la première lettre du thème écrite avec une lettre majuscule.

Le fait que Lu Mingu s'écrive avec un "L" n'est pas surprenant lorsqu'on sait qu'en anglais par exemple, les jours de la semaine commencent avec une lettre majuscule. Par contre, le fait que la première lettre $d u$ thème commence également avec une lettre majuscule peut soulever des interrogations. Un certain nombre d'exemples peuvent être cités par rapport au problème. Ceci peut être acceptable si l'auteur utilise cette notation à des fins stylistiques. Toutefois si cette notation doit être utilisée dans un dictionnaire par exemple, à coût sûr cela ne simplifiera pas la tâche aux usagers de ce système.

\section{POUR CONCLURE}

Les faiblesses observées au niveau orthographique sont problématiques pour l'usager. Comme cela apparaît plus haut, dans la littérature écrite sur le yipunu, le yilumbu et le civili il y a nombre de problèmes orthographiques qui doivent être résolus en prenant en compte les besoins et les aptitudes des usagers. C'est ce à quoi s'atèle la section suivante. 


\subsection{CONTRIBUTION DU PRESENT ARTICLE VERS UNE STANDARDISATION DE L'ORTHOGRAPHE DU YILUMBU, YIPUNU ET CIVILI}

\subsubsection{INTRODUCTION}

Les problèmes d'ordre orthographique sont nombreux, mais ceux liés à la division des mots, tradition du thème versus tradition du mot, mots composés avec trait d'union versus mots composés sans trait d'union, ainsi que l'écriture des variantes sont fondamentaux et par conséquent mérite un traitement spécial. Il convient également de mentionner que les propositions qui seront faites ici sont en accord avec les décisions du Rapport Final de la Session de Concertation sur l'Orthographe des Langues Gabonaises (1999).

\subsubsection{LE PROBLEME DES TONS}

Faut-il transcrire les tons? À cette question, Kwenzi-Mikala (1988) écrit ce qui suit:

C'est un peu comme si l'on se demandait s'il faut écrire les voyelles, ou les consonnes! Si l'on veut doter les langues gabonaises d'une graphie déchiffrable par tout un chacun, il est essentiel de noter les tons (Kwenzi-Mikala, 1988:209).

Cette citation de Kwenzi-Mikala confirme si besoin était l'importance de la transcription des tons dans les langues africaines et gabonaises en particulier. En outre, il existe une littérature abondante et variée sur l'importance capitale des faits suprasegmentaux ${ }^{10}$ (l'accent, l'intonation, les tons, etc.) dans la plupart des recherches linguistiques et lexicographiques.

Pour ma part, je dirai que d'un point de vue purement linguistique la prise en compte des phénomènes suprasegmentaux pour le yilumbu, le yipunu et le civili s'avère plus que nécessaire compte tenu du fait que ces parlers sont des langues à types tonales. En d'autres termes, le schème tonal d'un terme donné en isolation est susceptible de varier en fonction de sa position à l'intérieur d'une phrase. Concrètement sept classes tonales étiquetées $\mathrm{A}, \mathrm{B}, \mathrm{B}^{\prime}, \mathrm{C}, \mathrm{C}^{\prime}, \mathrm{D}$, et $\mathrm{D}^{\prime}$ ont été identifiées par Puech (1980) pour le yipunu. À partir d'items lexicaux en isolation, Blanchon et Nsuka Nkutsi (1984) ont identifié deux types tonals [H - H (B) et H - B (B)] pour le civili. Pour sa part, Mavoungou (2002c) a identifié deux catégories de substantifs en yilumbu, à savoir:

- Les substantifs qui subissent des perturbations tonales (Groupe 1).

- Les substantifs pour qui le schème tonal ne change pas quelque soit le contexte (Groupe 2).

10 Afane Otsaga (2002) propose quelques remarques sur la transcription suprasegmentale des textes oraux en langues africaines et gabonaises en particulier. Pour des informations plus complémentaires sur cet aspect, voir également Soami dans ce volume. 
Dans le Groupe 1, il convient également de faire la distinction entre les substantifs ayant un ton haut sur la première voyelle du thème en isolation et qui font surface avec un préfixe à ton haut dans d'autres contextes (Groupe 1a) et les substantifs ayant un ton bas sur la première voyelle du thème en isolation et qui font surface avec un préfixe à ton haut dans d'autres contextes (Groupe $1 \mathrm{~b}$ ). Les groupes 1 et 2 permettent donc d'identifier trois classes tonales pour le yilumbu:

Type $1: \mathrm{H}-\mathrm{H}$

Type 2: $\mathrm{H}-\mathrm{B}$

Type 3: B-B

Pour ce qui est de l'écriture orthographique des langues gabonaises, il est préférable de ne point noter les tons parce que le grand public n'est pas familier avec ces phénomènes suprasegmentaux. Pour ce qui est de la confection de dictionnaires en langues gabonaises, il est nécessaire que le traitement lexicographique de chaque article fasse ressortir la transcription phonétique des lemmes. Les différentes notations suivantes pourraient être utilisées:

Un ton très haut (TH) est noté par deux accents aigus: [v́]

Un ton haut $(\mathrm{H})$ est noté par un accent aigu: [v́]

Un ton bas (B) est noté par un accent grave: [v̀]

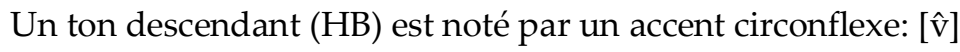

Un ton montant (BH) est noté par l'inflexe: [v̌]

\subsubsection{LE PROBLEME DE LA DIVISION DES MOTS}

Pour ce qui est de la division des mots, les langues africaines ont deux traditions d'écriture distincte, à savoir: une écriture conjonctive et une écriture disjonctive. Dans une écriture conjonctive, les mots présentent une structure interne complexe (par exemple les préfixes verbaux, les formatifs, les radicaux et leurs extensions forment un tout). Par contre, une écriture disjonctive présente une structure interne plus simple: les affixes (préfixes, infixes et suffixes) des items lexicaux apparaissent séparément (pour des informations plus détaillées lire Kwenzi-Mikala, 1988). Par rapport à ce point, Van Wyk (1995) a souligné que:

L'écriture conjonctive a eu l'avantage d'être vulgarisée par C.M. Doke qui a tenté de la justifier sur un plan phonologique. Par contre, aucun auteur n'a essayé de donner une assise théorique au disjonctivisme. Par conséquent, le conjonctivisme a été accepté de manière non critique par nombre de linguistes africains comme la seule méthode correcte sur le postulat non justifiée de l'existence d'une correspondance univoque entre l'écriture conjonctive et le "mot" défini de façon phonologique d'une part et les mots grammaticaux 
d'autre part. Il a été prouvé de façon conclusive que qu'il s'agisse du conjonctivisme ou du disjonctivisme, on a affaire à des conventions orthographiques ...L'écriture conjonctive a donnée naissance à la tradition du thème dans les langues du groupe Nguni et la tradition du mot dans les autres langues sud-africaines (Van Wyk, 1995:84, traduit par l'auteur).

L'analyse critique des dictionnaires disponibles dans les langues gabonaises révèle que les premiers travaux à caractère lexicographique ont adopté dans leur ensemble une écriture disjonctive. Les lexicographes travaillant à la confection de futurs dictionnaires dans les langues gabonaises gagneraient à perpétrer cette tradition. Pour le sujet qui m'occupe en particulier, je propose comme règle générale l'utilisation d'une écriture disjonctive pour le yilumbu, le yipunu et le civili en tenant compte des exceptions suivantes:

(a) Les préfixes (nominaux, verbaux, etc.) et les thèmes des items lexicaux doivent s'écrire conjointement.

Exemples:

(2)

\begin{tabular}{|c|c|c|c|c|c|c|}
\hline \multicolumn{2}{|c|}{ yipunu } & \multicolumn{2}{|c|}{ yilumbu } & \multicolumn{2}{|c|}{ civili } & \multirow{2}{*}{$\begin{array}{c}\text { Signification } \\
\text { en français }\end{array}$} \\
\hline $\begin{array}{l}\text { Transcription } \\
\text { orthographique }\end{array}$ & $\begin{array}{l}\text { Transcription } \\
\text { phonétique }\end{array}$ & $\begin{array}{l}\text { Transcription } \\
\text { orthographique }\end{array}$ & $\begin{array}{l}\text { Transcription } \\
\text { phonétique }\end{array}$ & $\begin{array}{l}\text { Transcription } \\
\text { orthographique }\end{array}$ & $\begin{array}{l}\text { Transcriptions } \\
\text { phonétiques }\end{array}$ & \\
\hline uji & [údzì] & uyi & [úyì] & kulya & [Kúlyà] & 'manger' \\
\hline bangebi & [bángèbì] & babaana & [bábâ:nə̀] & babaana & [bábâ:nə̀] & $\begin{array}{l}\text { 'petits } \\
\text { enfants' }\end{array}$ \\
\hline bataata & [bàtá:tè] & bataata & [bàtá:tə̀] & mataata & [mótá:tə̀] & 'pères' \\
\hline bamaama & [bàmá:mə̀] & bamaama & [bàmá:mò] & bamaama & [bə́má:mə̀] & 'mères' \\
\hline mwana & [mwâ:nə̀] & mwana & [mwâ:nə̀] & mwana & [mwâ:nə̀] & 'enfant' \\
\hline
\end{tabular}

(b) Les préfixes verbaux, les formatifs, les radicaux et leurs extensions devraient s'écrire de façon conjonctive: 


\section{Exemples:}

(3)

\begin{tabular}{|c|c|c|c|c|c|c|}
\hline \multicolumn{2}{|c|}{ yipunu } & \multicolumn{2}{|c|}{ yilumbu } & \multicolumn{2}{|c|}{ Civili } & significations \\
\hline Orth. & Phon. & Orth. & Phon. & Orth. & Pohn. & \\
\hline$\frac{\text { Amabokisa }}{\text { mwana. }}$ & $\begin{array}{c}\text { [àmábókìsə̀mw } \\
\text { â:nə̀] }\end{array}$ & $\frac{\text { Amabokisa }}{\text { mwana. }}$ & $\begin{array}{c}\text { [àmábókìsə̀mw } \\
\text { â:nə̀] }\end{array}$ & $\frac{\text { Avhondisa }}{\text { mwana. }}$ & $\begin{array}{l}\text { [àßóndìsə̀mw } \\
\text { â:nə̀] }\end{array}$ & $\begin{array}{l}\text { Elle a perdu un } \\
\text { enfant. }\end{array}$ \\
\hline$\frac{\text { Iibokyaanu! }}{\text { (kaari) }}$ & $\begin{array}{l}\text { [dzìbókyá:nù } \\
\text { (ká: rì)] }\end{array}$ & $\frac{\text { Mubokyaanu! }}{\text { (kaari) }}$ & $\begin{array}{l}\text { [mùbókyà:nù } \\
\text { (kă:rì)] }\end{array}$ & $\frac{\mathrm{N}^{\prime} \text { vhondyaa }}{\underline{\text { nu! (n'cima) }}}$ & [ńßóndyà:nù] & Tuez-le!(le singe) \\
\hline$\frac{\text { Twendyaanu }}{!}$ & [twendyá:nù] & Ndokwáánu! & [ndòkwá:nù] & Ndokwaanu! & [ndòkwá:nù] & Allons-nous-en! \\
\hline$\frac{\text { Amasyaala o }}{\text { nzima. }}$ & $\begin{array}{l}\text { [ámásyá:lèònzí } \\
\text { mə̀] }\end{array}$ & $\frac{\text { Anasyaala }}{\text { mbusa. }}$ & $\begin{array}{c}\text { [ámásyá:lèómb } \\
\text { ùsə̀] }\end{array}$ & $\frac{\text { Asyáála kú }}{\text { mbusa. }}$ & $\begin{array}{c}\text { [ásyá:làkúmb } \\
\text { ùsə̀] }\end{array}$ & $\begin{array}{l}\text { Il est resté } \\
\text { derrière. }\end{array}$ \\
\hline
\end{tabular}

(c) Les préfixes locatifs cl. 16 vha-et cl. $17 \mathrm{ku}$ - avec noms devraient s'écrire conjointement:

Exemples:

(4)

\begin{tabular}{|c|c|c|c|c|c|c|c|}
\hline & \multicolumn{2}{|c|}{ civili } & \multicolumn{2}{|c|}{ yilumbu } & \multicolumn{2}{|c|}{ yipunu } & \multirow[t]{2}{*}{ signification } \\
\hline & Orth. & Phon. & Orth. & Phon. & Orth. & Phon. & \\
\hline \multirow{2}{*}{$\begin{array}{c}\text { Classe } \\
16 \text { et } \\
\text { Classe } \\
17\end{array}$} & $\begin{array}{c}\text { kuyulu/kuyil } \\
\text { u ou encore } \\
\text { fayulu/fayilu }\end{array}$ & $\begin{array}{l}\text { [kìyŭlù/kùyı̌lù } \\
\text { fàyŭlù/fàyı̌lù] }\end{array}$ & $\begin{array}{l}\text { vhoyǐlu/ } \\
\text { vhayǐlu }\end{array}$ & $\begin{array}{l}\text { [ßòyîlù/ } \\
\text { ßàyı̌lù] }\end{array}$ & $\begin{array}{c}\text { ojịlu/ojŭ } \\
\text { lu }\end{array}$ & $\begin{array}{l}\text { [òdž̧lù/ } \\
\text { òdzŭlù] }\end{array}$ & $\begin{array}{l}\text { 'sur, au dessus } \\
\text { de; au ciel' }\end{array}$ \\
\hline & kusi/fasi & [kúsì/fásì] & $\begin{array}{c}\text { vhótsi/ } \\
\text { vhátsi/ótsi }\end{array}$ & $\begin{array}{c}\text { [ßótsì/ß } \\
\text { átsì/ótsì } \\
\text { ] }\end{array}$ & otsi & [ótsì] & $\begin{array}{l}\text { 'sur le sol, au sol; } \\
\text { en bas' }\end{array}$ \\
\hline \multirow[t]{2}{*}{$\begin{array}{c}\text { Classe } \\
16\end{array}$} & kukati/fakati & [kùkátì/fàkátì] & vhokáti & [ßòkátì] & oghari & [óghări] & $\begin{array}{l}\text { 'dans, dedans, à } \\
\text { l'intérieur de' }\end{array}$ \\
\hline & kunganda & [kùngâ:ndò] & $\begin{array}{c}\text { vhongând } \\
\text { a }\end{array}$ & $\begin{array}{l}\text { [ßòngâ: } \\
\text { ndò] }\end{array}$ & okana & [ókànə̀] & ‘dehors’ \\
\hline
\end{tabular}

(d) Les pronoms possessifs utilisés avec des termes de parenté devraient s'écrire de manière conjonctive. 


\section{Exemples:}

(5)

\begin{tabular}{|l|l|l|l|l|l|l|}
\hline \multicolumn{2}{|c|}{ yilumbu } & \multicolumn{2}{c|}{ yipunu } & \multicolumn{2}{c|}{ civili } & significations \\
\hline Orth. & Phon. & Orth. & Phon. & Orth. & Phon. & \\
\hline tayaami & [tàyá:mì] & tajaami & [tàdzá:mì] & lisyalyaami & [lísyályá:mì] & 'mon père' \\
\hline nguyaami & [ngùyá:mì] & ngujaami & [ngùdzá:mì] & nggulyaami & [ngùlyá:mì] & 'ma mère' \\
\hline$\underline{\text { nguyandi }}$ & [ngùyâ:ndì] & ngujandi & [ngùdzâ:ndi] & ngulyandi & [ngùlyâ:ndi]] & 'sa mère' \\
\hline
\end{tabular}

(e) Les pronoms démonstratifs utilisés avec des noms devraient s'écrire de façon conjonctive:

Exemples:

(6)

\begin{tabular}{|c|c|c|c|c|c|c|}
\hline \multicolumn{2}{|c|}{ yipunu } & \multicolumn{2}{|c|}{ yilumbu } & \multicolumn{2}{|c|}{ civili } & \multirow[t]{2}{*}{ signification } \\
\hline Orth. & Phon. & Orth. & Phon. & Orth. & Phon. & \\
\hline mutwaghughu & [mútwáyù] & mutwaghu & [mútwáyù] & mutwaghu & [mútwáyù] & 'cet homme' \\
\hline $\begin{array}{l}\text { mwanaghugh } \\
\mathrm{u}\end{array}$ & $\begin{array}{l}\text { [mwánə̀yúy } \\
\text { ù] }\end{array}$ & $\begin{array}{l}\text { mwanaagh } \\
\mathrm{u}\end{array}$ & [mwáná:yù] & $\begin{array}{l}\text { mwanaagh } \\
\mathrm{u}\end{array}$ & [mwáná:yù] & 'cet enfant' \\
\hline ndaghaaji & [ndâyádzì] & nzubwaayi & [nzùbwáyì] & nzwayi & [nzwâ:yì] & 'cette maison' \\
\hline
\end{tabular}

N.B. Bien qu'il ait été suggéré que le préfixe locatif de classe 16 vha- ou fa- avec noms s'écrive conjointement, les préfixes pronominaux o- ou encore ku-(classe 17) et mu- (classe 17) utilisés avec les noms ayant une valeur locative devraient s'écrire de façon disjonctive et non conjonctive. 
Exemples:

(7)

\begin{tabular}{|c|c|c|c|c|c|c|}
\hline \multicolumn{2}{|l|}{ civili } & \multicolumn{2}{|l|}{ yilumbu } & \multicolumn{2}{|l|}{ yipunu } & \multirow[t]{2}{*}{ signification } \\
\hline Orth. & Phon. & Orth. & Phon. & Orth. & Phon. & \\
\hline $\begin{array}{l}\mathrm{ku} \\
\text { lizandu/kuzand } \\
\mathrm{u}\end{array}$ & $\begin{array}{l}\text { [Kùlízà:ndù/k } \\
\text { úzà:ndù] }\end{array}$ & o yitanda & [òyìtándè] & o dikasa & [òdikásə̀] & 'au marché' \\
\hline ku n'situ & [kúńsìtù] & o musiru & [òmúsìrù] & o musiru & [òmúsìrù] & 'dans la forêt' \\
\hline $\begin{array}{l}\mathrm{ku} \\
\mathrm{meesu} / \text { kuntwaal } \\
\mathrm{a}\end{array}$ & [kùmé:sù] & o meesu & [òmé: sù] & o wuusu & [òwú:sù] & 'devant' \\
\hline ku mbusa & [kúmbùsə̀] & o mbusa & [ómbùsə̀] & o nzima & [ònzî:mə̀] & 'derrière' \\
\hline mu nzila & [mùnzílə] & mu nzila & [mùnzílè] & mu nzila & [mùnzílə̀] & $\begin{array}{ll}\text { ‘sur } & \text { le } \\
\text { chemin' }\end{array}$ \\
\hline
\end{tabular}

\section{POUR CONCLURE}

A partir de la discussion ci-dessus et par rapport aux règles de division de mots, la confection de tout dictionnaire en yilumbu, yipunu et civili devrait adopter de préférence une écriture disjonctive. Le choix entre une écriture disjonctive ou conjonctive est purement conventionnel. Pour clore la discussion sur ce concept de conjonctivisme versus disjonctivisme, $\mathrm{Nida}^{11}(1964)$ a fait remarquer que:

La longueur que peut atteindre nos verbes est alarmante. À cause du système des préfixes et des suffixes (du moins, ils sont considérés comme tels) nous combinons en de mots longs ce qui forme en anglais des phrases entières. Par exemple dans les Actes 10:7, on retrouve le terme abamamusalilanga 'ceux qui ont travaillé pour lui'. Il contient au moins trois préfixes et deux suffixes. (Le nombre dépend quelque peu du système d'analyse utilisé). Un tel terme semble extrêmement long, et on peut s'autoriser à penser que le système d'écriture utilisé gagnerait à introduire le plus de simplicité possible dans les graphies des mots. Pour nous qui ne sommes pas habitués à lire de longues unités linguistiques telles qu'on en trouve beaucoup dans les langues bantoues, de tels termes semblent être inutilement longs. Compte tenu $\mathrm{du}$ fait que nous en tant qu'analystes pouvons reconnaître les parties constituantes d'un mot et compte tenu du fait que certaines parties constituantes, les préfixes en particulier, correspondent à des mots

11 La contribution de Nida s'appuie sur le travail accompli par le Language and Literature Committee of the Christian and Missionary Alliance (CMA) sur le yipunu. 
entiers en anglais, il est naturel pour nous de chercher à segmenter des mots longs dans les langues bantoues. Toutefois, les préfixes: bamarquant le pluriel, ma- indiquant le temps passé et mu- troisième personne n'existent pas en isolation comme des mots complets. Ils sont à la fois phonologiquement et structurellement liés au radical verbal avec lequel ils apparaissent. Certains mots bantous semblent étonnement longs. Cependant, nombre de mots en Quechua et en Aymará, langues d'Amérique du Sud, sont de longueur analogue. Il n'est pas inhabituel de trouver en Quechua et en Aymará des mots de huit et dix syllabes sinon plus. En Aymará en particulier, il y a des mots longs avec combinaisons complexes de consonnes, mais malgré ce fait les locuteurs Aymará éprouvent relativement peu de difficulté à lire lesdits termes (Nida, 1964:154, traduit par l'auteur).

La section qui va suivre aborde quelques aspects de la tradition du mot par opposition à la tradition du thème.

\subsubsection{TRADITION DU MOT VERSUS TRADITION DU THEME}

La tradition du thème est souvent présentée comme étant plus scientifique et plus adaptée aux besoins des usagers que la tradition du mot pour essentiellement deux raisons:

- Cette méthode permet de regrouper les items lexicaux par affinités sémantiques,

- Elle évite certains secteurs de la nomenclature d'être surchargés pendant que d'autres sont presque vides.

Je pense, toutefois, que toutes ces explications ne sont pas des arguments suffisants pour arriver à confectionner un dictionnaire facile à consulter. Le fait que la majorité des dictionnaires disponibles dans les langues gabonaises ait adopté la tradition du mot pourrait placer les usagers dans une situation problématique si les items lexicaux sont lemmatisés sous la forme de leurs thèmes.

Emejulu et Nzang-Bie (1999b:12) ont souligné que dans les futurs dictionnaires confectionnés dans les langues gabonaises, les items lexicaux devraient être lemmatisés sous la forme de leurs thèmes. Toutefois, ils ne proposent aucune explication pour justifier un tel choix. Un bref aperçu des dictionnaires disponibles dans les langues gabonaises révèle que la tradition du mot est de loin la plus utilisée. Nyangone Assam et Mavoungou (2000) ont montré que de tous les premiers travaux à caractère lexicographique disponibles, seule la contribution de Rittaud-Hutinet (1980) lemmatise les items lexicaux du yipunu sous la forme du thème. 
Les dictionnaires confectionnés selon la tradition du thème sont souvent présentés comme étant plus facile à utiliser que les dictionnaires confectionnés selon la tradition du mot. Toutefois, l'adoption du système du thème pour les langues gabonaises pourrait créer une situation de confusion chez les usagers à cause de la tradition du mot qui existe déjà dans lesdites langues. Les dictionnaires confectionnés selon la tradition du thème pourraient même devenir impopulaire auprès des usagers en général (Van Wyk, 1995: 93).

De tels dictionnaires perdront assurément en accessibilité parce que requérant des acquis linguistiques non négligeables chez les utilisateurs des dictionnaires. Or l'on sait pertinemment que les usagers en général ont peu ou pas du tout d'expérience en linguistique (africaine). Ils ont encore moins une expérience dans la consultation des dictionnaires. Par conséquent, la consultation des dictionnaires confectionnés selon la tradition du thème risque de prendre beaucoup de temps parce que dans leur majorité lesdits usagers ne seront pas familiers avec la manière dont les données y seront présentées.

En sus, les usagers sont connus pour accorder peu ou pas du tout une attention à la lecture des pré-textes des dictionnaires (Busane, 1990: 28). Le guide à l'endroit des utilisateurs, en tant que seul type de texte obligatoire dans les pré-textes, devrait normalement renfermer le choix du/des lexicographe(s) pour l'adoption de la tradition du thème ainsi que sa justification. D'aucuns pourraient arguer que les lacunes des usagers consultant des dictionnaires confectionnés selon la tradition $\mathrm{du}$ thème pourraient être comblées par les mini-grammaires qu'ils contiennent.

Certes les mini-grammaires des dictionnaires donnent généralement un aperçu des règles morphophonologiques ainsi que des structures morphologiques de la langue décrite, mais encore faut-il que l'usager daigne les consulter. Or la tendance générale observée est que l'usager est beaucoup plus préoccupé d'aller vers la nomenclature que vers les pré-textes ou encore les posttextes du dictionnaire afin de trouver la réponse au problème qui a motivé le processus de consultation.

Si par contre, un lexicographe choisi d'adopter la tradition du mot, l'un des moyens pour arriver à produire un dictionnaire qui réponde aux besoins des spécialistes est d'utiliser la distinction entre texte externe intégré et texte externe non intégré développée par Bergenholtz, Tarp et Wiegand (1999). Cette distinction donne la possibilité au lexicographe de proposer un ensemble de types textuels ayant ou non un lien direct avec la nomenclature d'un dictionnaire spécifique. On peut par exemple dire que la 'Liste des contributeurs', 'La situation linguistique et sociolinguistique de la langue' décrite dans un dictionnaire particulier, 'Les tableaux des unités de mesure', etc. constituent des exemples typiques de textes externes non intégrés. 
A l'opposé, si au lieu d'inclure tous les mots présentant des difficultés orthographiques majeures pour les usagers, le lexicographe choisit de les traiter dans des posttextes, un tel type de texte sera un exemple de texte externe intégré. L'inclusion dudit texte dans les posttextes d'un dictionnaire particulier donnera à l'ouvrage un caractère poly-accessible. En fonction des besoins des usagers et la situation de communication, le processus de consultation pourrait être entamé à partir de la nomenclature ou alors à partir des posttextes. Si par exemple, un usager est confronté à l'orthographe d'un mot difficile mais n'a pas l'habitude de consulter des ouvrages de référence, l'inclusion de la liste orthographique des mots difficiles lui donne la possibilité d'aller directement vers les posttextes pour trouver une solution à son problème.

Cette distinction entre textes externes intégrés et textes externes non intégrés peut être mise à profit par le lexicographe confronté au problème du choix entre la tradition du thème et celle du mot. Il/elle peut par exemple adopter la tradition $\mathrm{du}$ mot tout en proposant aux usagers la liste de tous les thèmes des lemmes figurant dans la nomenclature. Toutefois, cette méthode a un inconvénient majeur: les usagers qui n'ont pas une connaissance des catégories grammaticales avant d'ouvrir le dictionnaire devront passer en revue l'ensemble des thèmes, l'un après l'autre, de ladite liste avant de trouver le thème qui les intéresse.

Par rapport à ce point la solution du dilemme tradition du mot/tradition du thème (auquel sont confrontés les lexicographes travaillant sur les langues africaines) réside dans la combinaison des aptitudes et des besoins à la fois des profanes et des spécialistes. En d'autres termes, la valeur du thème sera apparente pour le spécialiste mais d'une utilité très limitée pour l'usager ordinaire. Par conséquent, le meilleur moyen d'arriver à produire un dictionnaire facile à consulter est $\mathrm{d}$ 'adopter la tradition du mot tout en présentant, entre parenthèses, aux usagers les thèmes des lemmes traités comme présenté ci-dessous en (8):

(8)

mwana (-ana)...

Une telle approche ne doit pas être perçue comme une méthode destinée à donner aux usagers une connaissance des catégories grammaticales (parties du discours). C'est aux grammaires qu'il revient d'inculquer cette connaissance aux usagers, ce n'est pas le rôle du dictionnaire (Van Wyk, 1995:86). Kromann et al. (1991) partagent le même point de vue lorsqu'ils déclarent:

Il y a une division naturelle des tâches entre la grammaire et le dictionnaire. La grammaire a généralement pour tâche de formuler les règles générales régissant une catégorie grammaticale particulière, 
tandis que le dictionnaire s'occupe du mot (Kromann et al. 1991:2773, traduit par l'auteur).

Les données liées à la structure morphologique des items lexicaux sont utiles aux spécialistes, mais en cherchant un mot dans le dictionnaire l'usager ordinaire a principalement besoin de trouver des informations sur l'orthographe et le sens du terme consulté. Par conséquent, le thème donné entre parenthèses correspond à une méthode centrée sur l'usager. Elle est susceptible d'améliorer les connaissances linguistiques de l'usager aussi bien sur le plan de la réception que de la production sans être pour autant une clé pour la consultation dictionnairique.

Le fait que la tradition du thème est souvent présentée comme étant plus scientifique que la tradition du mot n'est pas dénué de tout fondement. En effet, Gouws (1990) souligne à ce propos que:

En limitant la portée linguistique du dictionnaire, le lexicographe restreint également sa valeur comme outil linguistique en ce sens qu'il y a peu de données exploitables par l'usager (Gouws, 1990: 55, traduit par l'auteur).

Il convient également de donner la position de Bennett (1986) sur la question:

Si les items sont alphabétisés par préfixes, certaines sections (de la nomenclature) sont surchargées pendant que d'autres sont presque vides, et un verbe sera inclus loin de ses dérivés nominaux et ce aussi transparents qu'ils soient. S'agissant des noms, L'opposition singulierpluriel est dans la majorité des cas marquée par l'emploi de préfixes distincts. Le degré de prédictibilité de la forme du singulier étant donnée la forme du pluriel et vice versa varie. Toutefois, il n'y a nulle part aucune prédictibilité totale (Bennett, 1986: 3-4, traduit par l'auteur).

Comme cela apparaît clairement dans la citation ci-dessus, si le lexicographe choisi seulement de présenter le singulier ou le pluriel pour chaque lemme du dictionnaire, il n'y a aucune garantie que l'usager sera en mesure de reconstruire la forme du pluriel étant donnée la forme du singulier et vice versa. Il est donc souhaitable de supprimer le préfixe pour ne garder que le thème et ce à cause des nombreux problèmes morphophonologiques qui en découleraient. Compte tenu du fait que la majorité des données morphologiques seront d'une utilité directe pour le spécialiste, la proposition d'alphabétiser les substantifs sous leur forme complète suivie du thème entre parenthèses résulte de la volonté du lexicographe de présenter une description morphologique rigoureuse de la langue. 


\section{POUR CONCLURE}

Par rapport au problème lié au thème et au numéro de classe, peut-être que la meilleure solution pour aboutir à un dictionnaire facile à consulter par le plus grand nombre est d'inclure ces catégories de données entre parenthèses. Ceci est pertinent en ce sens que, les parenthèses seront utilisées pour indiquer la partie facultative des données au niveau du commentaire sur la forme.

\subsubsection{LEMMATISATION DES VERBES ET DES ADJECTIFS (POSSESSIFS, DEMONSTRATIFS, ETC.)}

L'un des arguments contre la tradition du mot est que son adoption aboutit généralement à une surcharge de certaines sections de la nomenclature pendant que d'autres sont presque vides. Ceci est particulièrement vrai pour des catégories lexicales telles que les verbes, les adjectifs possessifs, les adjectifs démonstratifs, etc.

La solution à ce problème se situe dans la combinaison des caractéristiques à la fois de la tradition du mot et de celle du thème. Par exemple, De Schryver et Prinsloo (2000 : 9) ont souligné que dans le Lexicon Cilubà-Nederlands (1997, abrégé LNC), les verbes sont lemmatisés sous la forme de l'impératif comme cela apparaît clairement dans l'exemple qui suit:

-dyà [tww; cf spw3, 5] eten; kuukuta [ud] eten en verzadigdz bidyà; cidìlu; cyàkudyà; -dì̀ka; -dì̀iiibwa; -dì̀la; -dìsha; mudì; Mudì̀lampiku

Exemple 9 Article-dya (extrait de LCN tel que cité par De Schryver et Prinsloo, 2000: 9).

Selon De Schryver et Prinsloo (2000: 9) «l'approche traditionnelle qui consiste à ramener tous les verbes sous $k u / k w$ n'aboutit qu'à une surcharge artificielle d'une section du lexique en particulier». A la suite de LNC mais de façon quelque peu différente, dans le Pukuntšulhaloši sa Leboa 1.0 (PyaSsaL's First Parallel Dictionary, 2001) les verbes sont lemmatisés sous leur forme complète, tandis que les adverbes sont alphabétisés selon la tradition du thème. Comparez l'exemple suivant en guise d'illustration:

-nanalana lehlaodi BONA -nalana

Exemple 10: article -nanalana (extrait de PyaSsaL, 2001: 37)

Dans l'article ci-dessus, le traitement lexicographique fournit à l'usager les données suivantes, à savoir: le lemme (-nanala, sans son classificateur ku-), sa catégorie grammaticale ou partie du discours (lehlaodi = adverbe) ainsi que le renvoi (BONA - 
nalana). Les entrées (BONA = voir) et (-nalana) constituent deux segments de texte distincts. Le premier segment de texte est appelé le marquer de référence parce que son rôle est d'indiquer la relation de référence. Le second segment de texte est connu sous la dénomination d'entrée marquant l'adresse de référence.

\section{POUR CONCLURE}

La discussion ci-dessus a révélé que l'arrangement des lemmes demeure un sujet à controverse parmi les lexicographes bantouistes. L'analyse critique des dictionnaires disponibles dans les langues gabonaises révèle que les travaux précurseurs à caractère lexicographique ont adopté dans leur ensemble la tradition du mot. Les lexicographes travaillant à la confection de futurs dictionnaires dans les langues gabonaises gagneraient à perpétrer cette tradition.

\subsubsection{L'ORTHOGRAPHE DES MOTS COMPOSES}

La présentation des mots composés dans les dictionnaires souffre généralement d'inconsistance sur le plan orthographique. Faut-il écrire les mots composés avec un trait d'union ou sans trait d'union? Faut-il écrire les mots composés en deux mots ou en un seul mot?

Pour répondre à ces questions, des données supplémentaires sur la structure des mots composés méritent d'être présentées. D'un point de vue morphologique, les mots composés en yilumbu, yipunu et civili peuvent être classifiés en trois catégories, à savoir:

- les mots composés ayant la structure: nom + nom,

- les mots composés ayant la structure: dérivé + nom,

- les mots composés ayant la structure: nom + connectif + nom.

En anglais par exemple, d'un auteur à un autre un mot composé particulier s'écrira avec un trait d'union, en deux mots ou encore en un seul mot. Pour tous les mots composés appartenant aux trois catégories sus-mentionnées, je propose de les écrire en deux mots sans trait d'union. 


\section{Exemples:}

\begin{tabular}{|c|c|}
\hline \multirow[t]{3}{*}{ yilumbu et yipunu } & mwaana dibaala/dibaghala 'garçon' (structure: nom + nom) \\
\hline & yibura maambu 'provocateur, trouble-fête' (structure: dérivé + nom) \\
\hline & $\begin{array}{l}\text { dikuumbi di maamba 'hydravion; bateau' (structure: nom + connectif }+ \\
\text { nom) }\end{array}$ \\
\hline \multirow{3}{*}{$\begin{array}{l}\text { Transcriptions } \\
\text { phonétiques }\end{array}$} & [mwâ:nə̀dibă:lə̀/dìbáyə̀lə̀] \\
\hline & [yìbúrə̀mâ:mbù] \\
\hline & [dìkû:mbìdìmâ:mbə̀] \\
\hline \multirow[t]{3}{*}{ civili } & mwaana libakala 'garçon' (structure: nom + nom) \\
\hline & cibuta maambu 'provocateur, taquin' (structure: dérivé + nom) \\
\hline & likuumbi li masi 'hydravion; bateau' (structure: nom + connectif + nom) \\
\hline \multirow{3}{*}{$\begin{array}{l}\text { Transcriptions } \\
\text { phonétiques }\end{array}$} & [mwâ:nə̀líbákə̀lə̀] \\
\hline & [cìbútə̀mâ:mbù] \\
\hline & [lìkû:mbìlìmásì] \\
\hline
\end{tabular}

Toutefois, à cette règle, je propose également les exceptions suivantes:

(a) Je propose d'écrire en un seul mot tous les mots composés que les locuteurs natifs par intuition se représentent comme un bloc ${ }^{12}$.

Exemples:

\begin{tabular}{|c|c|c|c|c|c|c|}
\hline \multicolumn{2}{|c|}{ yipunu } & \multicolumn{2}{|c|}{ yilumbu } & \multicolumn{2}{|c|}{ civili } & \multirow[t]{2}{*}{ signification } \\
\hline Ortho. & Phon. & Orth. & Phon. & Orth. & Phon. & \\
\hline kumbukuku & $\begin{array}{l}\text { [kùmbúkùk } \\
\text { ù] }\end{array}$ & $\begin{array}{l}\text { kumbukuk } \\
\mathrm{u}\end{array}$ & $\begin{array}{l}\text { [kùmbúkùk } \\
\text { ù] }\end{array}$ & $\begin{array}{l}\text { kumbúkuk } \\
\text { u }\end{array}$ & $\begin{array}{l}\text { [kùmbúk } \\
\text { ùkù] }\end{array}$ & 'type de fourmi' \\
\hline $\begin{array}{l}\text { dighaghalab } \\
\text { onga }\end{array}$ & $\begin{array}{l}\text { [dìyáyə̀lə̀bô } \\
\text { :ngə̀] }\end{array}$ & $\begin{array}{l}\text { dighaghala } \\
\text { bonga }\end{array}$ & $\begin{array}{l}\text { [dìyáyə̀lə̀bô } \\
\text { :ngə̀] }\end{array}$ & $\begin{array}{l}\text { lighághalab } \\
\text { ônga }\end{array}$ & $\begin{array}{l}\text { [lìyáyə̀lòb } \\
\text { ô:ygə̀] }\end{array}$ & 'varan' \\
\hline $\begin{array}{l}\text { pwangapun } \\
\text { yi }\end{array}$ & $\begin{array}{l}\text { [pwâ:ygə̀p } \\
\text { únì] }\end{array}$ & pungapunyi & $\begin{array}{l}\text { [pú:ygə̀pún } \\
\text { i] }\end{array}$ & $\begin{array}{l}\text { pwenginipu } \\
\text { nyi }\end{array}$ & $\begin{array}{l}\text { [pwê:ngə̀ } \\
\text { púnì] }\end{array}$ & 'ogre' \\
\hline
\end{tabular}

(b) Je propose d'écrire les noms composés dénotant des anthroponymes avec un trait d'union.

12 Cette proposition, bien que plausible, présente également quelques insuffisances parce que basée sur l'intuition du lexicographe ou du linguiste. En effet, ce qu'un locuteur natif considère comme un bloc unique pourrait bien constituer deux blocs pour un autre. 


\section{Exemples:}

(13)

\begin{tabular}{|l|l|l|}
\hline $\begin{array}{c}\text { Graphies selon le Rapport Final de la } \\
\text { Session de Concertation sur l'Orthographe } \\
\text { des Langues Gabonaises (1999) }\end{array}$ & Transcriptions phonétiques & Graphies administratives \\
\hline Udighu-Ibinda & [ùdíyùibî:ndə̀] & Odigo Ibinda \\
\hline Miyindu-Mi-Nzamba & [mìyǔ:ndùmìnzâ:mbə̀] & Mihindou Mi Nzamba \\
\hline $\begin{array}{l}\text { Buro-Bu-Mbumba (forme abrégée de } \\
\text { Burobu-Bu-Mbumba) }\end{array}$ & $\begin{array}{l}\text { [bùróbùmbû:mbə̀/ } \\
\text { bùrŏbùbùmbû:mbò] }\end{array}$ & Bourobou Bu Mboumba \\
\hline Tshingombi-Kwima & [cìngô:mbìkwŭ:mə̀] & Tchingombi Kuima \\
\hline
\end{tabular}

\section{POUR CONCLURE}

La discussion ci-dessus présentée découle de la reconnaissance du fait que le problème de l'orthographe des mots composés doit être résolu longtemps à l'avance si on veut éviter des problèmes au niveau macro- et microstructurel par exemple.

\subsubsection{INDICATION DE LA QUANTITE VOCALIQUE}

En yilumbu, yipunu et civili, la quantité vocalique est phonologique. Elle est par conséquente marquée par le redoublement de la voyelle dans la majorité des cas. Toutefois, la longueur vocalique apparaissant dans le contexte VNC (position appuyée) n'est généralement pas indiquée orthographiquement. Hormis des exemples de resyllabification, la longueur vocalique se produisant dans le contexte CGV (la règle de MEEUSSEN) n'est pas reconnue.

\section{Contexte VNC}

(14)

\begin{tabular}{|c|c|c|c|c|}
\hline \multicolumn{2}{|c|}{ yilumbu/yipunu } & \multirow{2}{*}{\begin{tabular}{|l} 
significations \\
'arachide'
\end{tabular}} & \multicolumn{2}{|l|}{ civili } \\
\hline piinda & [pî:ndə̀] & & piinda & [phî̂:ndə̀] \\
\hline ditaambi & [dítà:mbi] & 'pied' & lutaambi & [lútà:mbì] \\
\hline dileengi & [dilĕ:ngì] & 'citrouille' & lileenjji & [lìlĕ:njì] \\
\hline
\end{tabular}

\section{Contexte CGV}

\begin{tabular}{|l|l|l|l|l|}
\hline \multicolumn{2}{|l|}{ yilumbu/civili } & significations & \multicolumn{2}{l|}{ yipunu } \\
\hline mwaana & [mwâ:ǹ̀] & 'enfant' & mwana & [mwâ:ǹ̀] \\
\hline
\end{tabular}




\begin{tabular}{|l|l|l|l|l|}
\hline cimweeka & [cìmwé:kè] & 'un' & moosi & [mô:sì] \\
\hline myaami & [myâ:mì] & 'mon, ma' (cl.4) & myaami & [myâ:mì] \\
\hline
\end{tabular}

A partir des données sus-mentionnées, il convient de noter que malgré le fait que la longueur vocalique apparaissant dans le contexte de la position appuyée (VNC) n'est généralement pas marquée orthographiquement une exception s'impose pour les cas d'allongements vocaliques prolongés. En sus, l'allongement vocalique se produisant lorsqu'une voyelle est redoublée ou triplée, etc. pour attirer l'attention du lecteur doit être prise en compte car elle a valeur d'effet de style ou de rhétorique.

\section{POUR CONCLURE}

La discussion ci-dessus résulte simplement de la reconnaissance de l'importance de l'allongement vocalique en yilumbu, yipunu et civili. En effet, la longueur vocalique étant phonologique dans les langues sus-mentionnées, elle doit être indiquée au niveau orthographique.

\subsubsection{LE PROBLEME DES VARIANTES}

Les sociolinguistes reconnaissent que généralement à l'intérieur d'un même dialecte il y a autant de d'idiolectes qu'il y a de locuteurs dudit dialecte. En outre, chaque acte de parole implique l'utilisation de différents codes ou registres en fonction de la compétence linguistique du locuteur ainsi que le contexte et la situation de communication (Crystal, 1992:69). Ce truisme est rappelé ici pour souligner l'importance de la prise en compte de toutes les variantes dans un dialecte particulier.

\subsubsection{LA PRISE EN COMPTE DES SONS [NZ] VERSUS [NTS] AU NIVEAU DE L'ORTHOGRAPHE}

Les sons [nz] et [nts] sont en variation libre ${ }^{13}$ dans nombre d'occurrences en yilumbu et yipunu. C'est devenu presque une norme d'écrire "nz" pour représenter les deux sons, et cela se produit principalement dans la littérature écrite du yilumbu, yipunu et civili. Le Dictionnaire français-yipounou, yipounou-français (1966) est une exception notable par rapport à ce point. En effet, dans ledit dictionnaire (lexique?), les membres du Language and Literature Committee of the Christian and Missionary Alliance (CMA) travaillant sur le yipunu ont choisi de représenter orthographiquement les sons [nz] et [nts] par le digraphe ns comme cela apparaît clairement dans le Tableau suivant:

13 En civili, les sons [nz] et [nz] sont en variation libre: mbenzza versus mbenzza "blessure". Les sons [nj] versus [n] et [s] versus [S] sont également en variation libre: $n$ 'lenji versus $n^{\prime}$ lenzi"cheveu"et mbisi versus $m b i f i$ "viande" respectivement, cf. Ndinga-Koumba-Binza (communication personnelle). 


\begin{tabular}{|c|c|c|c|}
\hline $\begin{array}{l}\text { Orthographe des items lexicaux } \\
\text { dans lesquels [nz] et [nts] sont } \\
\text { en variation libre comme ils } \\
\text { apparaissent dans CMA (1966) }\end{array}$ & $\begin{array}{l}\text { Graphies actuelles } \\
\text { des mêmes termes }\end{array}$ & $\begin{array}{l}\text { Transcriptions } \\
\text { phonétiques }\end{array}$ & Significations \\
\hline ngensa (mes caractères gras) & ngệnza & [ngê:nzè] & 'vrai; vérité' \\
\hline Nsala & nzála & [nzálè/ntsálè] & 'faim; famine' \\
\hline Nsambi & Nzâmbi & [nzâ:mbì/ntsâ:mbì] & 'Dieu' \\
\hline Nsanda & nzanda & [nzà:ndè] & 'toile d'araignée' \\
\hline Nsila & nzíla & [nzílà/ntsílà] & 'chemin; piste' \\
\hline Nsima & nzíma & [nzímə̀/ntsímə̀] & 'dos; après' \\
\hline Nsitu & nzíitu & [nzí:tù] & 'fin' \\
\hline nsonsi & nzônzi & [nzô:nzì/nzô:ntsì] & 'juge' \\
\hline nsungi & nzûngi & [nzû:ngì] & 'bile' \\
\hline nsungu & Nzûngu & [nzû:ngù/ntsû:Đgù]] & 'douleur; blessure' \\
\hline
\end{tabular}

Table 8: $\quad$ Orthographe items lexicaux dans lesquels [nz] and [nts] sont en variation libre

Dans le Tableau 8 ci-dessus, il faut souligner que l'orthographe de la majorité des termes extraits de CMA (1966) diffère de la graphie actuelle des mêmes mots parce que les auteurs ont choisi de représenter orthographiquement les sons [nz] et [nts] par le digraphe ns. C'est pour cette raison que certaines découvertes de Nsuka Nkutsi $^{14}$ (1980) ont été prises en compte dans la présente contribution pour aider le processus de standardisation et éviter la duplication d'efforts. Il n'est pas sans intérêt de mentionner l'existence de deux variantes pour le terme signifiant "Dieu". Nsambi ou Nzâmbi (graphie actuelle) est la variante utilisée en yilŭmbu yi ghângu (la variété de la province de la Nyanga) ainsi que dans le yipunu parlé dans la province de la Nyanga, spécialement à Tchibanga. Nzâmbi est également utilisé de manière proéminente dans les travaux liturgiques et bibliques en yipunu, yilumbu et Civili. À l'opposé, Nyămbi est la variante utilisée en yilŭmbu yí menaáne (la variété de la province de l'Ogooué-Maritime) ainsi qu'en yipunu (à l'exception de la variété parlée dans la province de la Nyanga).

Je propose donc d'utiliser le digraphe "nz" (représentant à la fois les sons [nz] et [nts]). Toutefois pour préserver et maintenir l'authenticité des formes dialectales

14 L'ouvrage Éléments de description du Punu édité par Nsuka Nkutsi est une série d'articles linguistiques et lexicographiques sur le yipunu. 
voire idiolectales, je propose de faire également apparaître la forme "nts" dans le traitement lexicographique. Ce point est illustré par ce qui suit ${ }^{15}$ :

Items lexicaux dans lesquels [nz] et [nts] sont en variation libre

nzala (+ zala) aussi ntsala [nzálà/ntsálə̀] $n$. (cl.9)....

-banza (+ bánza) aussi -bantsa [ùbá:nż̀/ùbá:ntsə̀] $n$. (cl. 15)...

Exemple textuel 16: article nzala et -banza

\section{POUR CONCLURE}

Comme cela apparaît dans la section ci-dessus, les sons [nz] et [nts] sont en variation libre dans nombre d'occurrences en yilumbu, yipunu et civili. La décision d'écrire le digraphe "nz" pour représenter les deux sons est importante pour des raisons de standardisation. Cette approche centrée sur l'usager est susceptible d'améliorer le processus de consultation dictionnairique.

\subsubsection{LA PRISE EN COMPTE DES SONS [MV] VERSUS [MF] AU NIVEAU DE L'ORTHOGRAPHE}

Les sons [mv] et [mf] sont en variation libre dans nombre d'occurrences en yilumbu et yipunu. La forme en "mv" est la plus répandue dans les descriptions linguistiques et les travaux en ethno-histoire. Pour des raisons de standardisation, il conviendrait d'utiliser le digraphe "mv" pour représenter les deux sons. Toutefois, le traitement lexicographique gagnerait aussi à faire ressortir la forme en "mf".

Items lexicaux dans lesquels [mv] et [mf] sont en variation libre

\begin{tabular}{|c|}
\hline yilumbu/yipunu \\
\hline 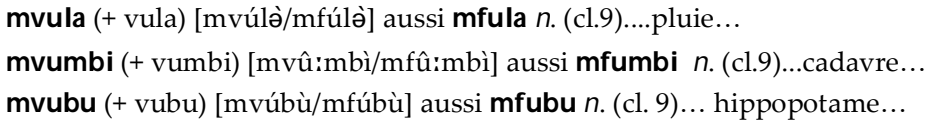 \\
\hline
\end{tabular}

Exemple textuel 17: article mvula, mvumbi et mvubu

15 Cette proposition n'est valable que dans le cadre de la confection du dictionnaire. Par rapport au concept de bi-univocité, pour tous les cas où les deux formes sont en variation libre le graphème standard est la forme [nz]. 


\section{EN GUISE DE CONCLUSION}

Cette contribution a essayé de faire l'exégèse de quelques travaux religieux et scientifiques disponibles en yilumbu, yipunu et civili avec en toile de fond des problèmes orthographiques. Conscient de l'influence normative des dictionnaires, cette contribution présente également un ensemble de propositions concrètes allant dans le sens de la standardisation du yilumbu, yipunu et civili. En d'autres termes, la présente contribution aborde divers aspects liés à l'alphabétisation des mots dans le dictionnaire (adoption de la tradition du mot par opposition à celle du thème), le problème des emprunts linguistiques, le choix entre une écriture conjonctive et une écriture disjonctive, le problème des tons, l'orthographe des mots composés et des termes dérivés, le problème des catégories lexicales (noms, verbes, adjectifs...) à lemmatiser, le problème des variantes, etc.

Par rapport à ce dernier point, le lexicographe devrait assister l'usager par rapport aux items lexicaux ayant des variantes concurrentes. Le choix d'une graphie à l'exclusion des variantes concurrentes a déjà d'importantes implications en termes de standardisation. Il est de la responsabilité du lexicographe de s'assurer que toutes les variantes sont correctement orthographiées dans le dictionnaire. Une ligne éditoriale consistante par rapport à ce point aidera non seulement l'usager mais également le processus de standardisation. Il n'est pas sans intérêt de préciser que la présente contribution n'avait pas pour objet de proposer un ensemble de critères devant aboutir au choix d'un dialecte standard pour le yilumbu, le yipunu ou encore le civili. Les dites formes standard restent à établir et elles seront en usage dans le système éducatif, les administrations et d'autres secteurs d'activité.

Bien que les exemples d'illustration dans cet article proviennent essentiellement du yilumbu, yipunu et civili, les différentes propositions linguistiques et métalexicographiques faites sont applicables à l'ensemble des langues gabonaises. En effet, l'objectif majeur de ce travail est que les linguistes, les lexicographes et le public en général arrivent à utiliser une orthographe unifiée. Pour ce qui est des transcriptions phonétiques, notre pays possède déjà l'Alphabet Scientifique des Langues du Gabon (ASG). La Fondation Raponda-Walker nous a montré la voie à suivre en 1995 lors de son S.O.S en faveur du lancement de l'apprentissage des langues gabonaises dans plusieurs établissements de la capitale et de l'intérieur du pays dès la rentrée 1996-1997.

Avec l'arrivée d'une nouvelle gamme d'outils didactiques (RAPIDOMATER, RAPIDOPREPA, RAPIDELEM et RAPIDOMOYEN), la Méthode RapidoLangue, a prouvée à suffisance qu'elle pouvait s'adapter aux réalités linguistiques du pays notamment par la reconnaissance de la quantité vocalique (marquée par le redoublement de la voyelle) et l'adoption du digraphe gh pour noter le son [x] telle que préconisée par le Rapport Final de la Session de Concertation sur l'Orthographe des langues Gabonaises (1999). 
Ce qui reste à faire c'est d'amener ceux et celles qui s'intéressent aux langues gabonaises à utiliser les symboles contenus dans le document sus-mentionné tout en leur laissant la latitude de la formulation de règles (d'écriture et de lecture) spécifiques à leur langue.

\section{REFERENCES}

\section{DICTIONNAIRES ET ENCYCLOPEDIES}

Bonneau, J. 1956. Grammaire pounoue et lexique pounoue-français, Brazzaville: Institut d'Études Centrafricaines.

Crystal, D. 1992. An Encyclopedic Dictionary of Language and Languages, Oxford: Blackwell Publishers.

De Schryver, G.-M. and Ngo. S. Kabuta. 1997. Lexicon Ciluba-Nederlands. Een circa 2500lemma's-tellend strikt alfabetish geordend vertalend aanleerderslexicon met decodeerfunctie teen behoeve van studenten Afrikaanse Talen en Culturen aan de Universiteit Gent, Ghent: Recall.

De Schryver, G.-M. (Ed.). 2001. Pukuntšuthaloši ya Sesotho sa Leboa 1.0 (PyaSsaL's First Parallel Dictionary), Pretoria: (SF)² Press.

Marichelle, C. 1900. Dictionnaire français-vili, Loango: Imprimerie de la Mission.

\section{AUTRES LITTERATURES}

Afane Otsaga, T. 2002. Les tons dans les dictionnaires de langues gabonaises: situation et perspectives. Lexikos 12: 75-89.

Afane Otsaga, T. 2004. The standard translation dictionary as an instrument in the standardisation of Fang. Unpublished D.Litt. Dissertation. Stellenbosch: University of Stellenbosch.

Alexandre P. 1961. Problèmes des états négro-africains à l'heure de l'indépendance. 2: 69-91.

Bendor-samuel, J. 1996. African Languages. Daniels, P., T. and W. Bright. (Eds.). The World's Writing Systems: 689-691. New York/Oxford: Oxford University Press.

Bergenholtz, H., S. Tarp et H. E. Wiegand. 1999. Datendistributionsstrukturen, Makro- und Mikrostrukturen in neueren Fachwörterbüchern. Hoffmann, L., H. Kalverkämper, H. E. Wiegand, together with Ch. Galinski, and W. Hüllen. Fachsprachen. Ein Internationales Handbuch zur Fachsprachenforschung und Terminologiewissenschaft / Languages for Special Purposes. An International Handbook of Special-language and Terminology Research: 1762-1832. Berlin/New York: Walter de Gruyter.

Blanchon, J.A. 1984. Présentation du yi-lumbu dans ses rapports avec le yi-punu et le ci-vili à travers un conte traditionnel. Pholia 1: 7-35, CRLS-Université Lumière Lyon 2.

Blanchon, J.A. et F. Nsuka Nkutsi. 1984. Détermination des classes tonales des nominaux en ci-vili, en i-sangu et en i-nzebi. Pholia 1: 37-45. CRLS-Université Lumière Lyon 2.

Busane, M. 1990. Lexicography in Central Africa: the User Perspective, with Special Reference to Zaïre". Hartmann, R.R.K. (Ed.) (1990): Lexicography in Africa: 19-35, Exeter: University of Exeter Press. 
Carpentier de Changy, H. \& M. Voltz. 1990. Alphabet Scientifique des Langues du Gabon (ASG)", Revue Gabonaise des Sciences de l'Homme 2:113-115.

De Schryver, G.-M. \& D. J. Prinsloo. 2000. The Concept of "Simultaneous Feedback": Towards a New Methodology For Compiling Dictionaries. Lexikos 10: 1-31.

Drame, A. 2000. Foreign Words as Problems in Standardisation/Lexicography: English and Afrikaans Loanwords in isiXhosa. Lexikos 10: 231-241.

Emejulu J.D. et Y. Nzang- Bie. 1999.“Initial Language Development”. Explaining Multilingual Education: Information on Some Tough Questions: 39-65.

Emejulu, J. et F. Pambo Loueya 1990. yilumbu. Revue Gabonaise des Sciences de l'Homme 2:197201.

Puech, G. 1980. La tonalité des thèmes nominaux en punu. Nsuka-Nkutsi, F. (Ed.). Éléments de description du Punu: 19-32, Lyon: PUL.

Garnier, A. 1897. Katesisa i gheghe nesi ma loghi ma dzambi mu mbembu i-lumbu, Loango: Imprimerie de la Mission.

Garnier, A. 1900.. Syllabaire i-lumbu keti mi ganda mio mi teti mi ranganga mu mbembo i lumbu, Loango: Imprimerie de la Mission.

Garnier, A.1904-. M'ambu ma nzambi mo make mu katesisa, Loango: Imprimerie de la Mission.

Gouws, R. H. 1990. Information categories in Dictionaries, with Special Reference to Southern Africa. Hartmann, R.R.K.(Ed.) (1990): Lexicography in Africa: 52-65, Exeter: University of Exeter Press.

Guthrie M. 1953. The Bantu Languages of Western Equatorial Africa, Oxford: Oxford University Press.

Hartmann, R.R.K. (Ed.) 1990. Lexicography in Africa. Progress Reports from the Dictionary Research Centre Workshop at Exeter, 24-26 March 1989, Exeter Linguistic Studies Volume. 15, Exeter: University of Exeter Press.

Hausmann, F. J. et H. E. Wiegand. 1989. Component Parts and Structures of General Monolingual Dictionaries: A Survey. Hausmann, F. J. et al. (Eds.). 1989-1991. Wörterbücher.Ein Internationales Handbuch zur Lexikographie/ Dictionaries. An International Encyclopedia of Lexicography/Dictionnaires. Encyclopédie Internationale de Lexicographie: 328-360, Berlin: Walter de Gruyter.

Hausmann, F. J., Reichmann, O., Wiegand, H. E. and L. Zgusta. (Eds.). 1989-1991. Wörterbücher. Ein Internationales Handbüch zur Lexikographie/ Dictionaries. An International Encyclopedia of Lexicography/Dictionnaires. Encyclopédie Internationale de Lexicographie. 3 volumes; 3355 pages, Berlin: Walter de Gruyter.

Ilson, R. F. (Ed.). 1985. Dictionaries: Lexicography and Language Teaching. ELT Documents 120. Oxford: Pergamon Press.

Kidda Awak. 1990. Historical Background, with Special Reference to Western Africa Hartmann, R.R.K.(Ed.) (1990): Lexicography in Africa: 8-18. Exeter. University of Exeter Press.

Kromann, H.-P., T. Riiber, P. Rosbach. 1991. Grammatical Constructions in Bilingual Dictionaries. F. J. Hausmann et al. (Eds.), Volume III. 2770-5.

Kwenzi-Mikala, J. T. 1980. Contes punu du Gabon: Etude linguistique et sémiologique. Thèse de $3^{\text {ième }}$ cycle, Lyon: Université Lumière Lyon 2, 693 p.

Kwenzi-Mikala, J. T. 1988. Quelques remarques sur la transcription des textes oraux en langues africaines. Pholia 3: 207-211, CRLS-Université Lumière. Lyon 2. 
Kwenzi-Mikala, J. T. 1989. Contribution à l'analyse des emprunts nominaux du yipunu au français. Pholia4:157-170, CRLS-Université Lumière Lyon 2.

Kwenzi-Mikala, J. T. 1990 L'anthroponymie chez les Bapunu du Sud-Gabon. Pholia 5:113120, CRLS-Université Lumière Lyon 2.

Kwenzi-Mikala, J. T. 1998a- Parémies d'Afrique Centrales. Libreville: Éditions RapondaWalker.

Kwenzi-Mikala, J. T. 1998b- Mumbwanga. Libreville: Editions Raponda-Walker.

Le Testu, G. 1918. Notes sur les Coutumes Bapounou dans la Circonscription de la Nyanga. Caen, Haulard la Brière.209 p.

Mabika Mbokou, L. 1999. Les phénomènes analogiques en civili: Etude phonologique et morphologique, Mémoire de maîtrise, Université Omar Bongo, Libreville

Mavoungou, P.A. 2001a. Macro- and Microstructural Issues in Mazuna Lexicography. Lexikos 11: 122-138.

Mavoungou, P.A. 2001b La Mondialisation et la Lexicographie Trilingue ou Plurilingue au Gabon. Emejulu J. D. (Éd.). 2001: Eléments de Lexicographie Gabonaise Tome 1: 160183, New York: Jimacs-Hillman Publishers.

Mavoungou, P.A. 2002a Synopsis Articles in the Planning of a Trilingual Dictionary: Yilumbu-English-French. Lexikos 12: 181-200.

Mavoungou, P.A. 2002b. Sociolinguistic and linguistic Aspects of Borrowings in Yilumbu. South African Journal of African Languages 22 (1): 41-58.

Mavoungou, P.A. 2002c. Metalexicographical criteria for the compilation of a trilingual dictionary: Yilumbu-English-French. Thèse de Doctorat non publiée. Stellenbosch: Université de Stellenbosch.

Mavoungou, P.A. T. Afane Otsaga and G.-R. Mihindou. 2002c. Dictionaries Compiled with French and the Reproduction of the Gabonese Cultures. Paper presented at the Seventh International Conference of the African Association for Lexicography, organized by the Dictionary Unit of South African English, Grahamstown University, 7-10 July 2002.

Mini, B. M. 1995. Lexicographical Problems in isiXhosa. Lexikos 5: 40-56.

Ministère de l'Éducation Nationale. 1999. Rapport Final de la Session de Concertation sur l'Orthographe des langues Gabonaises, document inédit.

Murard, P. (trad.). 1903. Katsisu i keki i rendilu mu mbembo bis'Sette-Cama (petit catéchisme), Lyon: Imprimerie Paquet, p.53.

Murard, P. 1903. Katsisu i neni i rendilu mu mbembo bis'Sette-Cama (grand catéchisme), Lyon: Imprimerie Paquet, p.135.

Nida, E.A. 1950. Orthographic Problems in Yipounou. Bible translator 1:110-116. Reedited by William A. Smalley et al. 1964:148-155, London: United Bible Societies.

Ndamba, J. 1977. Syntagme nominal et groupe nominal en vili (H12): langue du Congo, Thèse de doctorat $3^{\text {ième }}$ cycle, Paris: Université de Paris 3.

Ndinga-Koumba-Binza, H.S. 2000. Phonologie du civili de Mayumba, Mémoire de maîtrise, Université Omar Bongo, Libreville

Nyangone Assam et Mavoungou, P.A. 2000. Lexicography in Gabon: A Survey. Lexikos 10: 252-274.

Nzang-Bie, Y. 2001. Vers une Éducation Multilingue au Gabon: Première Approche. Revue Gabonaise des Sciences du Langage/Gabonese Journal of the Language Sciences 2: 17-29. 
Puech, G. 1990. “Bekwel”. Revue Gabonaise des Sciences de l'Homme 2: 127-128.

Raponda-Walker, A. 1932. Alphabet des idiomes gabonais". Journal de la Société des Africanistes 3(2): 305-314.

Raponda-Walker, A. 1933. Les néologismes dans les idiomes du Gabon. Journal de la société des Africanistes 3(2): 305-314. Réédité dans Langues du Gabon, Editions RapondaWalker, Classiques Africains, 222 p.

Sacleux, Ch. 1905. Essai de phonétique, Paris. Procure.

Touré, A. 1990. L'écriture des langues africaines: évolution et principes méthodologiques. Revue Gabonaise des Sciences de l'Homme2: 55-63.

Van Wyk, E.B. 1995. Linguistic Assumptions and Lexicographic Traditions in the African Languages. Lexikos 5: 82-96.

Watch, G. 1993. Institut du Christ-Roi Souverain Prêtre, Paris: Maison Bienheureux Daniel Brotier.

Wiegand, H.E. 1998. Wörterbuchforschung, Berlin: Walter de Gruyter. 


\title{
LA Place DES TONS DANS \\ L'ORTHOGRAPHE DES LANGUES \\ GABONAISES
}

\author{
LEANDRE SERGE SOAMI \\ INSTITUT DE RECHERCHE EN SCIENCES HUMAINES (IRSH) \\ Centre National de Recherche Scientifique et Technologique (CENAREST), Libreville \\ (sergesoami@gmail.com)
}

\section{INTRODUCTION}

Les tons jouent un rôle important dans le fonctionnement des langues bantu en général et dans les langues gabonaises ${ }^{1}$ en particulier. Pour les besoins de l'enseignement et du développement général de ses langues locales, le Gabon a amorcé depuis 1989, avec le Séminaire pour un alphabet scientifique des langues nationales, la mise en place d'un système d'écriture de ces langues. Ledit séminaire avait établi l'Alphabet Scientifique des Langues du Gabon : (ASG).

Mais à la différence des autres phénomènes suprasegmentaux, les tons sont très problématiques dans la confection d'un système orthographique des langues gabonaises. Le débat porte sur la question de savoir si l'on doit noter les tons dans l'orthographe des langues gabonaises. Ce débat n'est pas encore arrivé à ses conclusions dès lors que l'alphabet des langues gabonaises n'est pas encore adopté par les pouvoirs publics, et que le système orthographique de ces langues n'est pas encore rigoureusement établi².

Il faut tout de même rappeler que le ton est l'élévation ou la diminution de la voix sur une syllabe. C'est une phase de la hauteur de la voix qui peut être pertinente sur le plan sémantique.

L'objectif de ce travail est d'apporter une contribution à la solution de ce problème et de faire le point du traitement de la tonalité dans le système orthographique des langues gabonaises. Pour ce faire, il est important de revenir de manière générale sur les systèmes tonals de nos langues et sur la problématique que pose leur

L'abréviation LG est mise en certains endroits pour Langues Gabonaises ou Langues du Gabon.

Voir Afane Otsaga (dans cet ouvrage) pour la distinction entre alphabet et système d'orthographe. 
graphie. Ceci permettra d'observer s'il est nécessaire ou non d'orthographier les tons des langues gabonaises.

\section{GENERALITE SUR LES SYSTEMES TONALS DES LANGUES GABONAISES}

$C^{\prime}$ est un truisme que de dire que les langues gabonaises sont des langues à tons, c'est-à-dire des langues "où une commutation phonologiquement limitée à la hauteur ou à la mélodie d'une syllabe unique est susceptible de constituer la seule trace d'une commutation entre deux unités significatives minimales dans une construction inchangée" (Creissels 1994: 175). Autrement dit, l'opposition de deux ou trois tons distinctifs engendre un changement de sens. On note les exemples ciaprès:

\section{(9) OPPOSITIONS DISTINCTIVES DE QUELQUES TONS EN LG.}

/ngülì/ «bâton»3 vs / Đgúlì/ «mère» (civili)

/bwámú/ «bon» vs /bwámū/ «le bien» (benga)

/ggüjì/ "potamochère" vs /ggújì/ "mère" (yipunu)

Ainsi, le ton a dans chaque langue gabonaise une fonction distinctive. En effet, chaque syllabe des termes de ces langues est caractérisée sur le plan phonologique par un ton ou une séquence de tons distincts (Matthews, 1997: 379). Les experts du séminaire sur l'ASG ont identifié les neuf tons, présentés ci-dessous, comme pertinents dans l'ensemble des langues gabonaises (Carpentier de Changy et Voltz 1990 : 114).

\section{(10) LES NEUF TONS DES LANGUES GABONAISES SELON L'ASG.}

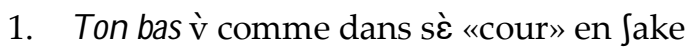

2. Ton moyen $\overline{\mathrm{v}}$ comme dans íyól $\bar{\varepsilon}$ «pouvoir» en benga

3. Ton haut v́ comme dans dólì «argent» en yipunu

4. Ton descendant $\hat{\mathrm{v}}$ comme dans mbô «bras» en sake

5. Ton montant v̌ comme dans díkǒn «lance» en seki

6. Ton très haut v" comme dans ngüyì «sanglier» en gisira

7. Ton très bas v̈ comme dans wülú «pied» en lendumu

8. Ton haut abaissé vं comme dans múti «arbre» en liduma

9. Ton très haut descendant ṽ comme dans jgáânzì «racines» en yipunu

3 D'après Ndinga-Koumba-Binza (communication personnelle), ce mot semble être l'un des rares mots en civili portant un ton supra haut. 
La plupart de ces tons ont dans l'ensemble des langues gabonaises des fonctions distinctives significatives, aussi bien dans le système phonologique que dans le vocabulaire.

D’une manière générale, les tons sont regroupés en deux catégories distinctes : les tons ponctuels et les tons modulés. Un ton ponctuel est celui qui, systématiquement fixé sur une unité syllabique, s'identifie par un seul aspect de la courbe mélodique : haut ou bas. Le ton modulé par contre se distingue par des changements successifs de la courbe mélodique dans la réalisation d'une syllabe. Les tons ponctuels sont les plus récurrents dans les langues gabonaises.

Dans certaines langues tel que le benga, on retrouve pour l'essentiel des tons ponctuels. Essono (1990: 138-139) identifie pour le benga trois tons ponctuels phonologiques récurrents (le ton bas, le ton moyen et le ton haut) et un seul ton modulé, le ton montant, extrêmement rare que l'auteur trouve dans le terme ínǒnì. D'autres langues à l'instar du civili (Blanchon \& Nsuka Nkutsi 1984, Blanchon 1990, Ndinga-Koumba-Binza 2000) connaissent une variation complémentaire entre les tons ponctuels et les tons modulés. Ndinga-Koumba-Binza (2002: 115-116, 2002) indique pour le civili que l'allongement vocalique produit comme corrélat au niveau du système tonal une modulation de la courbe mélodique par l'adjonction d'un ton laissé libre sur une voyelle résiduelle. Ce qui montre que dans certaines langues gabonaises les tons modulés sont dus à des processus à la fois phonologiques et tonologiques.

Comme le dit Afane Otsaga (2002:77), «les différentes descriptions réalisées jusqu'à ce jour sur les langues gabonaises ont identifié dans celles-ci l'existence de trois catégories de tons». Il s'agit des tons principaux, des tons intermédiaires et des tons secondaires.

Les tons principaux sont les tons les plus récurrents, ce sont essentiellement les tons ponctuels haut et bas et les tons modulés montant et descendant. Ils ont une large diffusion dans les langues gabonaises.

Le ton haut, représenté par un accent aigu sur la voyelle (v́), est le point le plus haut de la courbe mélodique, tandis que le ton bas (ì) est le plus bas de la courbe.

\section{(11) EXEMPLES ET OPPOSITIONS TON HAUT VS TON BAS}

/díkó/ «source»vs /díkò/ «sud» (benga)

/mvám/ «grand-père» vs /mvàm/ «générosité» (fang-ntumu)

/góré/ «il y a» vs /górè/ «chez» (myenè-nkomi)

Dans la réalisation du ton montant, la courbe descend d'abord au point le plus bas, puis remonte au point le plus haut sur une même syllabe qui peut être longue ou non. 


\section{(12) EXEMPLES DU TON MONTANT}

/íßâsììi/ «ils acceptèrent» (lekaningi)

/jwă/ «être trempé de sueur» (lembaama-lempiini)

/mwôsì/ «tous» (lendumu)

Le ton modulé descendant est par contre le mouvement de la courbe mélodique qui monte vers le point le plus haut, puis redescend vers le point le plus bas sur une même syllabe brève ou longue.

\section{(13) EXEMPLES DU TON DESCENDANT}

/ipíkîlyà/ «les pensées» (myenè-mpongwè)

/mâ:kò/ «vin» (benga)

/ع́tây/ «lire» (seki)

Les tons intermédiaires sont «les tons les moins récurrents et les moins répartis dans les langues gabonaises que les tons principaux, mais dont la présence dans ces langues n'est pas négligeable puisque facilement remarquable» (Afane Otsaga 2002:78). Parmi ces tons intermédiaires, l'on note le ton ponctuel moyen, le ton haut-abaissé et le ton supra-haut. Le ton moyen se présente au milieu de la courbe mélodique, entre le point le plus bas et le point le plus haut. On le retrouve dans les mots de la langue fang (variante nzaman de $\mathrm{Makokou}^{4}$ ) comme:

\section{(14) EXEMPLES DU TON MOYEN}

$/ \bar{n} l \bar{\jmath} /$ «mouche»

/3ụ̄/ «gros bois de chauffage»

/n̄zōn/ «chemin, piste»

Le ton haut-abaissé est le résultat du phénomène de faille tonale. Hombert (1990: 102) explique que ce type de ton est réalisé «phonétiquement à un niveau qui peut être assimilable au ton». Ce qui le distingue du 'véritable' ton moyen est le fait que le ton haut-abaissé ne puisse être suivi par un ton plus haut. Constituant ainsi un plafond pour tous les tons suivants du mot ou du syntagme, il est suivi par un terrassement tonal, «c'est-à-dire que la série des tons hauts qui suit la faille tonale est réalisée au même niveau que le ton haut abaissé» (Afane Otsaga, 2002:78). D'après Hombert (1990: 103), «la plupart des langues du Gabon connaissent ce phénomène de faille tonale». L'on note les exemples suivants en lembaama et en ngubi.

4 Ces exemples sont extraits de Cinnamon (1990: 181). 


\section{(15) EXEMPLES DU TON HAUT-ABAISSE}

[mèma'áta'] «'ai tiré» (lembaama)

[íngu'yì] «mère» (ngubi)

Parmi les tons intermédiaires il y a également le ton supra-haut ou très haut que l'on retrouve dans les langues comme le yipunu et le lekaningi. C'est un ton ponctuel qui sur le plan phonétique «a comme point de départ une valeur plus élevée qu'un ton haut et qui ensuite chute très rapidement » (Hombert 1990: 03). L'on observe le ton très haut dans les exemples ci-après.

\section{(16) EXEMPLES DU TON TRES HAUT}

[ùläb̀̀] «voir» (yipunu)

[säsì] «appât» (lekaningi)

Les tons secondaires sont «les tons assez rares dans les langues gabonaises et dont la répartition entre ces dernières est véritablement infime». Dans ce groupe on note les tons infra-bas ou très bas et le ton très haut descendant. Le ton très bas est l'opposé $\mathrm{du}$ ton très haut dans la courbe mélodique. Sa courbe est descendante. "Il s'agit donc d'un ton bas réalisé plus bas que le ton bas normal "(Afane Otsaga 2002: 79). Hombert (1990: 102) précise qu'il existe parfois un contraste entre un ton bas ponctuel et un infra-bas. Mais il est fréquent que cette distinction ne se produise que dans certains contextes, par exemple avant la pause.

\section{(17) EXEMPLES DU TON INFRA-BAS}

[wülù] «pied» (lendumu)

[wümà] «combattre» (meka)

Le ton très haut descendant est également très rare dans les langues gabonaises. Sa définition est encore imprécise. Il semble être un ton supra-haut suivi d'un ton bas sur une même syllabe. L'exemple unique qui est cité de ce ton vient du yipunu.

\section{(18) EXEMPLE DU TON TRES HAUT DESCENDANT}

ngáânzi «racines» (yipunu)

A ce jour, le recensement des particularités tonales dans les langues gabonaises est loin d'être exhaustif. Ceci pour deux raisons. D'abord, toutes les langues gabonaises n'ont pas encore été décrites. De plus, des études spécifiques des langues gabonaises tenant compte à la fois des faits tonétiques et des processus tonologiques sont rarement abordées. Ce qui fait qu'on n'a pas une vue minutieuse et spécifique de chaque système tonal des langues gabonaises. 
Toutefois, cet examen général donne un certain aperçu des réalités tonales dans les langues gabonaises. Le nombre assez important des tons observés implique pour certains auteurs, que «leur influence dans le fonctionnement de ces langues est sans conteste à prendre en compte» (Afane Otsaga, 2002:79). La prise en compte de la tonalité dans le système orthographique des langues gabonaises fait l'objet d'une problématique dans les cercles linguistiques au Gabon.

\section{LA PROBLEMATIQUE DE LA NOTATION DES TONS DANS LES LANGUES DU GABON}

Dans le cadre d'une écriture spécifique aux langues gabonaises en fonction de leurs spécificités structurelles, certains auteurs soutiennent la transcription des tons dans le système d'orthographe de ces langues. Mais des réserves par rapport à cette position sont souvent formulées. Par exemple, la notation des tons surchargerait les textes d'accents.

\section{LES RAISONS DE LA NOTATION DES TONS}

Pour Kwenzi Mikala (1988: 209), par exemple, «si l'on veut doter les langues gabonaises d'une graphie déchiffrable par tout un chacun, il est essentiel de noter les tons». Pour Daniel F. Idiata (2002: 61), le problème de la notation des tons ne se pose pas quand il écrit: «les tons doivent être transcrits, parce qu'ils jouent un rôle aussi important que les consonnes ou les voyelles». Car, ainsi que le précisent d'autres auteurs, transcrits seulement sur le plan segmental, les textes en langues gabonaises sont difficiles à lire même pour les locuteurs dont c'est la langue maternelle. Kwenzi Mikala (1988: 209) suggère de contourner la difficulté, dans une certaine mesure, «en ne notant rien que le ton le plus fréquent (en général le ton bas)».

La vraie question de la notation des tons, selon Idiata (2002: 61), est « comment faire pour arriver à transcrire les tons d'une manière qui soit la plus économique et la plus fonctionnelle possible?». Pour l'auteur, la réponse à cette question dépend non seulement de la spécificité de la langue mais aussi du destinataire du texte. Idiata (2002: 62) recommande que le problème de la notation des tons soit traité au cas par cas. C'est-à-dire que chaque langue devra être dotée d'un système particulier quant à la notation des tons, compte tenu du fait qu'il y a «des systèmes plus complexes que d'autres, comme il y a des systèmes plus faciles que d'autres». Finalement, au lieu d'une dynamique d'ensemble des langues gabonaises comme pour les graphèmes consonantiques et vocaliques, la notation des tons devra se constituer en un système diversifié selon les langues.

Par ailleurs, la Session de Concertation sur l'Orthographe des Langues Gabonaises d'avril 1999 a admis le principe de la notation des tons. Cependant, les experts de cette Session ont recommandé, pour l'écriture de ces tons, la mise en place des commissions d'étude et d'harmonisation des différents systèmes linguistiques 
existants. L'application effective de cette recommandation se fait toujours attendre. Néanmoins, pour l'assignation tonale les cinq diacritiques (suscrits) ci-dessous furent retenus par les experts.

\section{(19) DIACRITIQUES POUR L'ASSIGNATION TONALE (AVRIL 1999)}

le ton haut est indiqué par le signe de l'accent aigu (')

le ton bas est indiqué par le signe de l'accent grave ( ' )

le ton moyen est indiqué par la barre horizontale $\left(^{-}\right)$

le ton modulé descendant est indiqué par le signe de l'accent circonflexe $(\wedge)$

le ton modulé montant est indiqué par le signe de l'accent circonflexe renversé ( ).

L'on peut conclure cette section par la remarque selon laquelle la Session de Concertation a finalement admis la notation, en général, de cinq tons des systèmes tonals des langues gabonaises. Il s'agit du ton haut, du ton bas, du ton montant et du ton descendant.

\section{DES PREOCCUPATIONS QUANT A LA NOTATION DES TONS}

Au-delà de la simple surcharge des textes, il y a bien d'autres raisons sur lesquelles, la notation des tons achoppe.

\section{EST-IL UTILE DE NOTER LES TONS RARES?}

J'ai déjà souligné qu'il existe une diversité de tons rares d'une langue à l'autre. Je pense que dans le cadre de la notation des tons, il n'est pas sans intérêt de prendre en compte les tons rares. C'est-à-dire, des tons auxquels les locuteurs eux-mêmes ne prêtent pas forcément attention. A ce sujet, le nouvel alphabet des langues gabonaises adopté à la Session de Concertation évoquée plus haut est d'une avancée très significative.

De plus, il faut noter que la majorité de ces tons rares (aussi bien intermédiaires que secondaires) sont en variation avec des tons ponctuels ou sont tout simplement une succession de ces tons ponctuels. En liwanzi (Mwélé 1990: 253) et en yisangu (Ondo-Mebiame 1988, 1990) par exemple tous les tons modulés sont une séquence des tons ponctuels haut et bas (ou bas et haut) sur une même voyelle longue ou brève ou sur une séquence de voyelles. Ainsi des tons tels que le ton très bas descendant du yipunu et le ton infra-bas sont automatiquement écartés de toute possibilité de transcription orthographique. 


\section{A-T-ON PEUR DES HOMONYMES?}

Les arguments en faveur de la notation des tons reposent en gros sur le fait que les tons jouent un rôle distinctif dans le vocabulaire des langues gabonaises, comme d'ailleurs dans toutes les langues à tons. Ceux-ci réfutent l'idée qui préconise qu'avec l'absence des tons l'on peut s'appuyer sur le contexte pour identifier le contour tonal du mot. En effet, lorsqu' on prend en compte les paires minimales des tons on peut conclure à des situations homophoniques si le pallier suprasegmental n'est pas considérés. Retenir le principe d'homophonie impliquerait que le sens d'un mot peut être identifié selon le contexte dans lequel le mot est employé.

La qualification des paires minimales suprasegmentales est difficile pour bon nombre de chercheurs impliqués dans la sémantique, la confection des dictionnaires et la linguistique appliquée (Nzang-Bie 2000: 136, Sanogo 2001: 115, Afane Otsaga 2001). Pour Denis Creissels (1994:173), "Il s'agit là d'une organisation de la courbe mélodique qu'il est impossible de réduire à des choix entre unités discrètes, avec des variations qui dépendent directement de la structure communicative du message et non pas des unités significatives minimales sélectionnées pour en assurer la formulation. L'importance de ce phénomène dans la communication orale est considérable, mais sa spécificité gêne beaucoup le linguiste...». Il s'agit en d'autres termes du fonctionnement linguistique de la hauteur de la voix dans le cadre du mot. Ce fonctionnement est généralement un phénomène de ton, quand il n'est pas seulement un fait général de l'intonation.

Le problème des linguistes a généralement été d'une part d'identifier ou non ces mots qui se distinguent seulement au niveau prosodique comme des homophones (ou homonymes en général quand on tient compte de leur graphie aussi souvent identique) et d'autre part de qualifier la relation psychosémantique de ces mêmes items linguistiques. En ce qui concerne la relation d'homophonie, il est clair qu'elle n'est pas du tout posée, puisque, comme le montre Ndinga-Koumba-Binza (op.cit) dans le cadre du civili, la prononciation du mot est une combinaison de deux paliers: le palier des segments (consonnes et voyelles) et le palier des faits prosodiques (tons, intonation, accent, etc.).

Le ton ${ }^{6}$ qui distingue les mots de la paire minimale est donc l'un des éléments du niveau prosodique. Il doit sa réalisation en tant qu'élément phonique à l'ensemble prosodique même du mot. De ce fait, il est à conclure qu'il ne saurait y avoir des cas d'homophonie dans une paire minimale (bantu) pour ton. Mavoungou \& Ndinga-Koumba-Binza (2010) évoque pour le civili que des cas d'homophonie stricts, c'est-à-dire des mots ayant une même structure segmentale et une même

\footnotetext{
Ndinga-Koumba-Binza (en préparation)

6 Pour certains auteurs tels que Ndinga-Koumba-Binza (op.cit) le concept de tonème est superfétatoire dans le cadre des langues à tons. Car si l'on dit qu'une langue est une langue à tons cela implique le caractère distinctif de ces tons dans la langue.
} 
structure tonale, apparaissent dans les langues seulement dans le cadre des emprunts linguistiques et des termes génériques. Ce qui pose encore la nécessité du contexte pour la distinction significative des mots.

Ainsi, quand bien même le principe d'homophonie ne serait pas admis pour les paires minimales des tons, il reste que dans le phénomène du contexte il peut amener à distinguer des mots bantu identiques. Si l'on arrive à distinguer des homophones purs, c'est qu'il sera également possible de distinguer par le contexte les mots dont la réalisation phonétique diffère au niveau tonal. On a les exemples ci-après en civili.

\section{(20)CAS D'HOMOPHONES STRICTS EN CIVILI}

/cikóóku/ «réliques» vs /cikóóku/ «dance punu» (emprunt au yipunu)

/múnù/ «bouche»vs /múnù/ «toute forme d'entrée» (générique)

Ces mots ne se distingueront qu'en fonction du contexte, à la fois du contexte du discours (le sujet du discours ou de la communication) et du contexte grammatical (dans la phrase). Finalement, c'est la prise en compte de toutes les formes de contextes qui donne le sens au mot prononcé ou écrit. Le terme nguji par exemple a le sens de mère dans un contexte discours religieux (nguji Nyaambi, la mère de Dieu) ou de parenté (nguji bootsu, la mère de tous).

\section{L'EXEMPLE DES AUTRES PAYS AFRICAINS.}

Il faut d'abord noter que les langues à tons sont très nombreuses dans le monde. La plupart des langues asiatiques (le japonais, le mandarin, etc.) et bon nombre des langues autochtones d'Amérique par exemple sont des langues à tons. Leurs systèmes sont souvent plus complexes que ceux des langues bantu en général, et des langues gabonaises en particulier.

En ce qui concerne la notation des tons, bon nombre de pays ont eu à retenir une écriture simple qui ne tient pas compte des tons. C'est le cas de tous les pays d'Afrique australe (Namibie, Botswana, Afrique du Sud, Swaziland, etc.) et des pays d'Afrique de l'Est (Kenya, Tanzanie, Soudan, etc.). En Afrique centrale, les pays comme le Rwanda, le Burundi, l'Ouganda et la République Démocratique du Congo ont des systèmes d'écriture dans laquelle la tonalité des langues n'est pas prise en compte. Des langues de grande expansion comme le hausa en Afrique de l'Ouest et le Swahili parlé à la fois en Afrique centrale (Rwanda, République Démocratique du Congo) et en Afrique de l'Est s'écrivent également sans notation des paramètres suprasegmentaux. Il faut également préciser que la tradition écrite des langues africaines dans bon nombre de ces pays est séculaire. C'est le cas de toutes les langues sud-africaines, du swahili, du lingala et du ciluba dont la 
tradition écrite remonte depuis le XIIIème siècle. Les figures ci-dessous présentent des textes écrits dans certaines langues africaines.

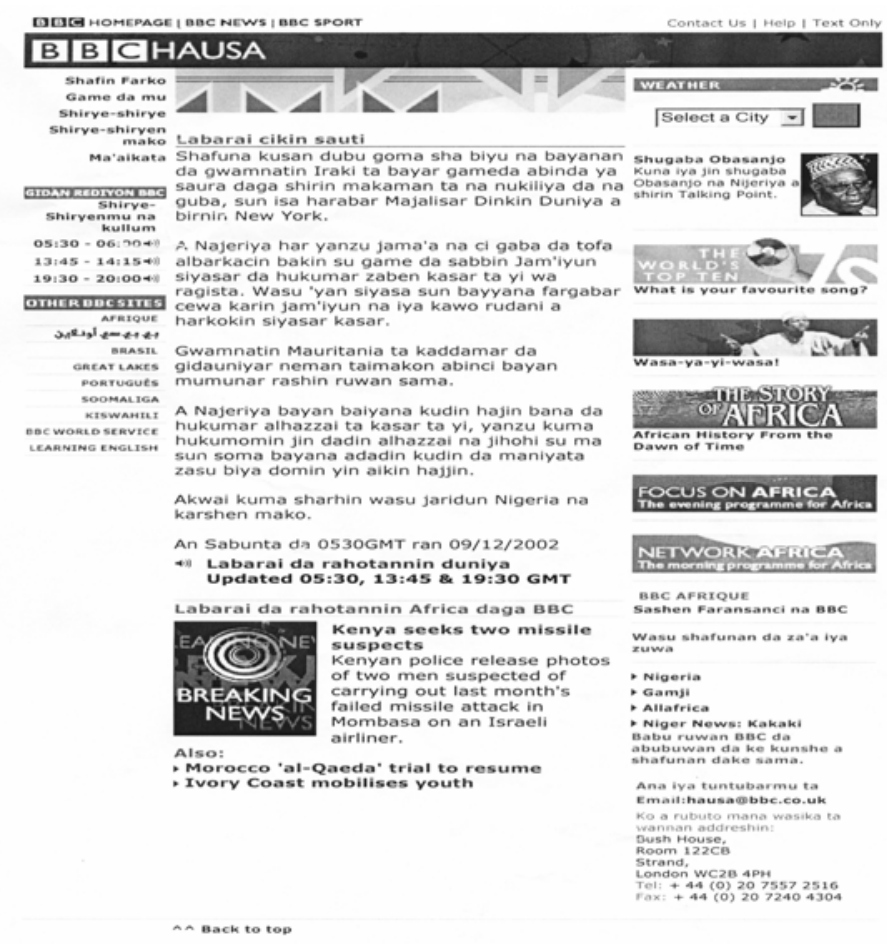

Figure 1: Extrait de texte hausa (http://www.bbc.co.uk/hausa/

Le hausa, langue tchadique du phylum afro-asiatique, a sur le plan phonémique deux tons principaux - le ton haut et le ton bas - et un ton secondaire, le ton descendant. L'intonation a également une grande importance dans la langue. Cependant, aucun phénomène prosodique n'est pris en compte dans le système orthographique. 


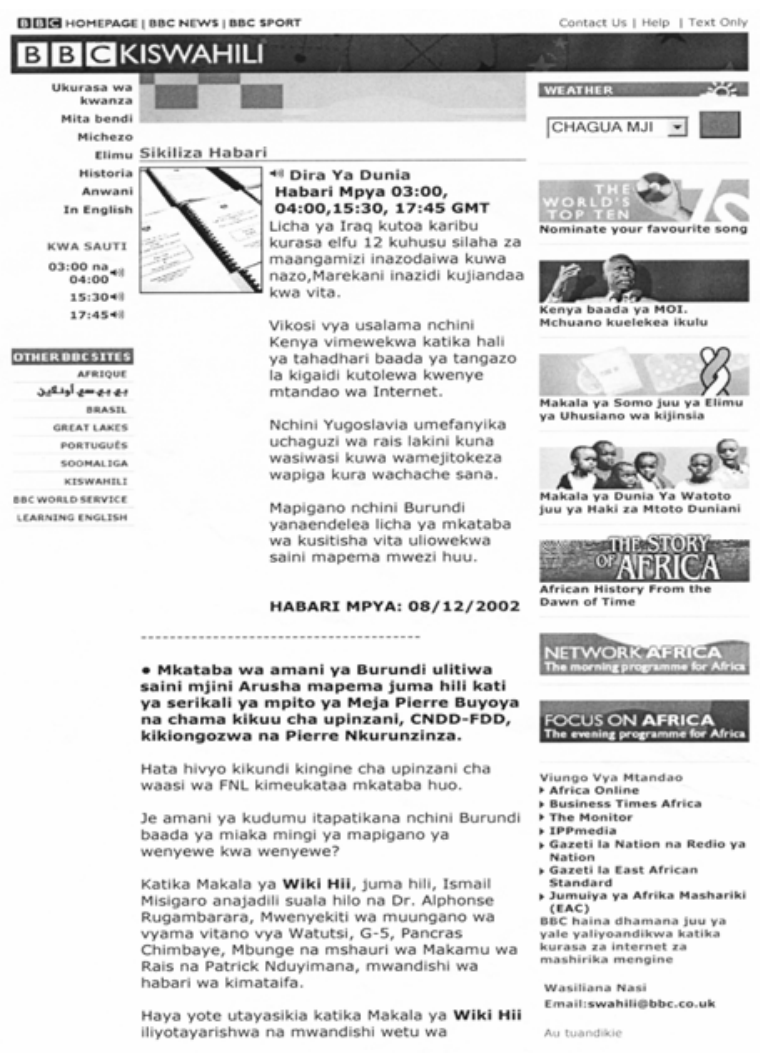

Figure 2: $\quad$ Extrait de texte kiswahili (http://www.bbc.co.uk/swahili/

Le kiswahili, langue bantu exceptionnellement sans ton, était jusqu'à la fin du IXème siècle écrit avec l'écriture arabe. Sa romanisation actuelle qui reprend toutes les lettres de l'alphabet de la langue anglaise (sauf $c$, q et $\mathrm{x}$ ) ne note aucun phénomène suprasegmental. 

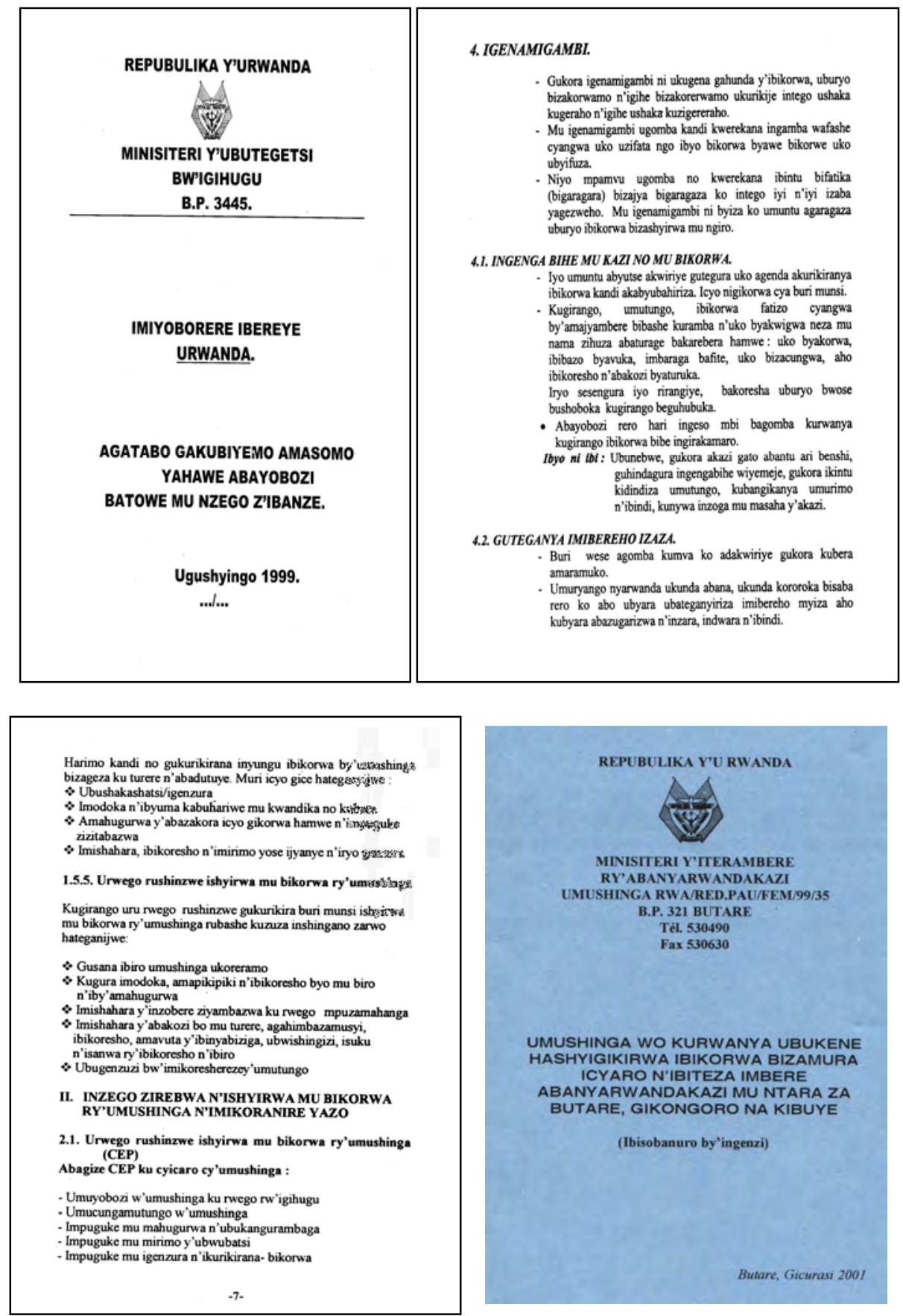

Figure 3: Extrait de textes kinyarwanda

Le kinyarwanda est une langue à tons. Comme le kiswahili duquel s'inspire son système orthographique (le swahili est avec l'anglais et le français l'une des quatre 
langues officielles de la République Rwandaise), le kinyarwanda ne note pas les phénomènes suprasegmentaux.

Par ailleurs, des langues comme le tswana ont eu à changer plusieurs fois leur système d'écriture en ce qui concerne la transcription tonale (Cole \& Mokaila 1962). En effet, avec les missionnaires et administrateurs coloniaux jusqu'à l'orée du XXème siècle, l'écriture du tswana ne tenait pas compte des tons. A partir de la deuxième moitié du même siècle, les tons furent introduits dans le système d'orthographe de la langue. Malgré l'adoption de cette disposition par de nombreuses littératures tswana (romans, journaux, etc.), elle ne résista pas aux difficultés qui se posaient au grand public. Le tswana dut revenir dès les années 1970 à une écriture sans indication tonale.

En ce qui me concerne, en matière de développement des langues en général et du problème des indications tonales, le Gabon gagnerait à s'inspirer des pays qui ont déjà assis une tradition écrite de leurs langues. Comme le dit Idiata (2002: 59) «il serait simplement modeste de s'inspirer de leur échecs et surtout de leurs réussites».

\section{DE LA REACTION DES POPULATIONS}

La prolifération des tons dans les textes de langues peut, comme les signes diacritiques de l'alphabet, provoquer une certaine réticence des populations locales qui dans toute leur majorité sont profanes des sciences du langage. Il faut noter que la plupart des travaux qui ont élaboré des propositions d'alphabets et/ou d'orthographes se sont beaucoup plus focalisés dans la recherche de la qualité scientifique et de l'objectivité, oubliant parfois de tenir compte du grand public profane. On sait dans le monde entier comment des grandes idées scientifiquement révolutionnaires ont échoué ou, sont peu pratiqués faute de légitimité sociale. Le clonage humain par exemple est l'une des grandes révolutions scientifiques de ces temps-ci, mais sa corruption de la conscience sociale et de la moralité humaine le laisse impopulaire et légalement interdit.

A mon avis, la scientificité quelque peu exagérée des propositions déjà faites tel que l'ASG, ou même encore le nouvel alphabet des langues gabonaises, serait d'une certaine manière l'une des raisons qui ralentit la prise de décision des pouvoirs publics à adopter et promulguer les recommandations des différents séminaires et autres sessions d'où sont issues les différentes propositions. Car les pouvoirs publics ne vont pas adopter une proposition, quoique scientifique, dont les populations seront réfractaires.

\section{LA FAIBLESSE DES TONS DANS L'APPRENTISSAGE D'UNE LANGUE}

L'apprentissage d'une langue commence rarement par les phénomènes suprasegmentaux. Les missionnaires par exemple comme beaucoup d'Européens ont appris des langues africaines, et des langues bantu en particulier, comme 
seconde ou troisième sans aucun obstacle au niveau tonal. Raponda-Walker (1998:83-84) fait référence des efforts de ces Européens en ces termes :

"C'est de voir le plus grand nombre possible de coloniaux, fonctionnaires, commerçants et exploitants forestiers, s'efforcer d'apprendre l'une ou l'autre langue indigène, de manière à pouvoir comprendre les Noirs et se faire comprendre directement d'eux sans le secours d'un interprète... Pourquoi les missionnaires, tant catholiques que protestants parviennent-ils plus facilement à comprendre l'indigène et à se faire comprendre de lui, sinon par suite de la connaissance qu'ils ont acquise du parler de leurs ouailles ? Mais cette connaissance, absolument nécessaire pour l'enseignement religieux, $n^{\prime}$ est pas moins utile à la bonne administration du pays qu'aux tractations commerciales ou autres qui mettent les Blancs en relation journalière avec les Noirs ».

Je pense que si des locuteurs d'autres langues sont arrivés à apprendre les systèmes tonals de nos langues sans que ceux-ci soient transcrits, il en sera de même pour tout autre individu. En effet, l'apprentissage du système tonal comme de l'ensemble prosodique d'une langue est généralement et d'abord inconscient pour des locuteurs non natifs comme pour des locuteurs natifs. La maîtrise des règles quand elle se pose n'apparaît que bien plus tard.

\section{UNE EXPERIENCE DE RAPIDOLANGUE}

Dans le cas de nombreuses langues africaines, comme dans le cas particulier des langues gabonaises, il est avéré que les tons semblent rébarbatifs aussi bien pour les élèves que pour les enseignants. Cette situation fut observée à l'issue d'une expérience personnelle dans le cadre de l'expérimentation de la méthode Rapidolangue de la Fondation Raponda-Walker ${ }^{7}$. Parmi les enseignants, bon nombre étaient et sont des étudiants du DSL qui ont une certaine connaissance des structures tonales des langues gabonaises. Ainsi, les tentatives des enseignants de faire prendre en compte les tons dans la lecture et l'écriture par les élèves se sont souvent soldées par une réticence totale de ces derniers. En effet, les élèves trouvaient une difficulté quant à l'apprentissage (de lecture comme d'écriture) de tons du fait de l'habitude qu'ils ont du français et de l'anglais qui sont des langues dont l'écriture est relativement simple et ne nécessite pas la notation des accents sur toutes les voyelles. La maîtrise des systèmes tonals n'est donc pas une chose aisée chez les élèves.

Il faut également relever que le système éducatif gabonais part du préscolaire au supérieur. De ce fait, va-t-on aussi demander aux enfants de moins de 3 ans qui

7 L'auteur a servi pendant trois ans (de 1998 à 2001) à la Fondation Raponda-Walker en qualité d'enseignant de la langue yipunu à l'Institution Immaculée Conception, au Collège Sainte Marie et au Collège Notre Dame de Quaben. 
apprennent la langue, à retenir la transcription des tons que son éducatrice ellemême n'est pas sure de maîtriser?

\section{DES IMPLICATIONS DE RECHERCHES SCIENTIFIQUES}

L'on peut voir des points qui précèdent que le débat de la graphie des tons peut soulever des questionnements plus larges que la simple scientificité et l'objectivité que l'on veut donner à l'écriture des langues gabonaises. En effet, si les tons doivent être orthographiés, il va falloir un long processus d'explication et de véhicule, au sein des populations, de la notion des tons et des méthodes de transcription des tons. Chaque ton dans chaque langue devra être suffisamment décrypté, sur le plan scientifique au travers des méthodes de la tonétique et de tonologie expérimentale, en vue d'un discernement nécessaire chez tous les locuteurs qui sont tous sensés l'écrire et le lire correctement. Ceci implique de longues, minutieuses et rigoureuses recherches tonétiques et tonologiques des langues gabonaises.

Par ailleurs, s'il faut tenir compte des aspects commerciaux et économiques, je doute fort que Microsoft puisse se permettre de créer un clavier particulier pour une langue africaine. Car sur le plan capitaliste il n'y a aucun intérêt. Si cela était possible, cela aurait été déjà fait dans les pays qui ont à ce jour un niveau de développement assez élevé de leurs langues. L'expérience en matière de recherche linguistique en langues africaines a montré les limites des programmes informatiques contenant les signes diacritiques particuliers contenus dans les langues bantu en générales et gabonaises en particulier. Transcrire ou saisir des textes de ces langues n'est pas chose facile car il faut passer des heures entières pour achever un travail qui prendrait peu de temps en utilisant Microsoft Word ou tout autre programme du même type.

La SIL International a dans certaines langues bantu créé des programmes et polices phonétiques, à l'instar de Cam Cam SIL Doulos (pour les langues camerounaises), pour tenter de lier par des raccourcis les symboles particuliers de ces langues aux touches du clavier d'ordinateur. Mais de tels systèmes nécessitent également une importante vulgarisation auprès des populations. De plus ils ne résolvent pas nécessairement la difficulté des saisies informatiques et traitements de textes sur les systèmes d'applications de Microsoft (Word, Excel, Frontpage, etc.) puisqu'il $\mathrm{s}^{\prime}$ agit de polices nouvelles en forme de caractères spéciaux. Ils ne résolvent pas non plus le temps qu'on a à insérer des caractères spéciaux.

\section{L'INSTABILITE DU SYSTEME TONAL}

Enfin, il faut souligner que les systèmes tonals des langues sont en constante évolution. L'argument de la tonogénèse est que les langues perdent beaucoup plus qu'elles n'en créent. C'est-à-dire que plus évolue une langue plus son système 
tonal tend à se simplifier. En d'autres termes, les langues perdent les tons au fur et à mesure qu'elles se développent ou qu'elles sont au contact d'autres systèmes linguistiques. C'est le cas du swahili qui, à ce jour, ne relève plus aucune distinction tonale significative. Le swahili est aujourd'hui de manière exceptionnelle une langue bantu sans ton.

Sur le plan synchronique l'instabilité des systèmes tonals des langues se distingue par l'organisation de certains de ces systèmes en types tonals. C'est le cas de bon nombre des langues gabonaises parmi lesquelles le civili (Blanchon \& Nsuka Nkutsi 1994, Ndinga-Koumba-Binza), le yisangu (Ondo-Mebiame 1988, 1990) et le wumvu (Blanchon 1989, Rekanga 2000). Dans la plupart de ces langues, les mots de vocabulaire peuvent être regroupés en classes tonales. De plus, la tonalité d'un mot est en même de changer selon la position (en isolation ou en contexte) ou la fonction (sujet ou objet) de ce mot dans la phrase.

Ce phénomène de types tonals, en tant qu'il est une certaine variation sans le système de la langue, peut être considéré sur le plan diachronique comme un processus initial aux changements au niveau de la tonalité des langues. C'est-à-dire que la tonalité peut subir des variations complexes au point de perdre le caractère distinctif. De ce fait, on peut d'une certaine manière préméditer la perte des tons dans certaines langues gabonaises à types tonals 8 .

\section{CONCLUSION}

Cet article vient de revenir sur le débat qui porte sur la transcription orthographique des tons dans les langues gabonaises. On peut retenir que le caractère distinctif des tons n'est pas une raison suffisante pour admettre la prise en compte de ces tons dans le système d'orthographe des langues. De nombreuses raisons aussi bien linguistiques qu'extralinguistiques favorisent plutôt une écriture simple sans indication tonale. C'est ce qui se fait dans la majorité des pays (aussi bien africains que non africains) aux contextes proches du nôtre. La perspective qui réfute la graphie des tons s'appuie sur la considération du grand public qui a seul le pouvoir social de légitimer toute proposition scientifique faite à son objectif.

Les recherches linguistiques en Afrique de manière générale auront tout à gagner en tenant compte aussi bien des besoins que des aptitudes des locuteurs respectifs de ces langues en processus de développement.

\section{REFERENCES}

Afane Otsaga, T. 2002. Les tons dans les dictionnaires des langues gabonaises: situation et perspectives. Lexikos 12: 75-89. Stellenbosch: Buro van die WAT.

8 Des études spécifiques de tonologie nécessitent d'être mené dans ce sens. 
Blanchon J.A. 1989. Le wumvu de Malinga (Gabon): tonalité des nominaux. Pholia 6: 39-44. . CRLS-Université Lyon.

Blanchon, JA. \& Nsuka Nkutsi, F. 1994. Détermination des classes tonals des nominaux en ci-vili, en i-sangu et en i-nzebi. Pholia 1: CRLS-Université Lyon 2.

Carpentier de Changy, H. et Voltz, M. 1990. Alphabet scientifique des langues du Gabon. Revue Gabonaise des Sciences de l'Homme 2 : 113-115. Libreville : LUTO/Publications de l'Université Omar Bongo.

Cinnamon, J. 1990. Fang (nzaman). Revue Gabonaise des Sciences de l'Homme 2: 175-183. Libreville : LUTO/Publications de l'Université Omar Bongo.

Cole, D.T. and Mokaila, D.M. 1962. A course in Tswana. Washington: Georgetown University.

Creissels, D. 1994. Aperçu sur les structures phonologiques des langues négro-africaines. Grenoble: Ellug.

Essono, H. 1990. Benga. Revue Gabonaise des Sciences de l'Homme 2: 129-139. Libreville: LUTO/Publications de l'Université Omar Bongo.

Kwenzi Mikala, JT. 1988. Quelques remarques sur la transcription des textes oraux en langues africaines. Pholia 3: 207-211.

Matthews, PH. 1997. Concise dictionary of linguistics. Oxford/New York: Oxford University Press.

Mavoungou, P.A. \& Ndinga-Koumba-Binza, H.S. Civili, langue des Baloango: esquisse historique et linguistique. Munich: Lincom Europa.

Ministère de l'Education Nationale. 1999. Rapport Final de la Session de Concertation sur l'Orthographe des Langues Gabonaises. Libreville.

Mwélé, M. 1990. Liwanzi. Revue Gabonaise des Sciences de l'Homme 2 : 251-255. Libreville: LUTO/Publications de l’Université Omar Bongo.

Ndinga-Koumba-Binza, HS. [En préparation]. Homophonic creations in Civili.

Ndinga-Koumba-Binza, HS. 2000. Phonologie du civili de Mayumba: langue bantu du Gabon. Libreville : Université Omar Bongo. Mémoire de Maîtrise.

Ndinga-Koumba-Binza, HS. 2004. Vowel duration issue in Civili. South African Journal of African Languages 24(3): 189-201.

Nzang-Bie, Y. 2000. Les phénomènes tonals dans le syntagme nominal de détermination: le cas des langues bantu du nord ouest. Revue Gabonaise des Sciences du Langage 1: 135-149.

Ondo Mebiame, P. 1988. Esquisse de description du yisangu, langue Bantu de la zone B, de sigle B42. Mémoire de Licence spéciale. Université Libre de Bruxelles.

Ondo Mebiame, P. 1990. Yisangu. Revue Gabonaise des Sciences de l'Homme 2 : 307-318. Libreville : LUTO/Publications de l'Université Omar Bongo.

Rekanga, J.P. 2000. La tonalité des nominaux en wumvu. Bulletin Ndaga 2: 26-32. NDAGA/Publications de l'Université Omar Bongo.

Sanogo, T. 2001. Contribution à l'étude du système tonal du Jula. Revue Gabonaise des Sciences du Langage 2: 114-124. 



\title{
UNITES-LANGUES ET STANDARDISATION DANS LES LANGUES GABONAISES
}

\author{
Hugues STEVE NDINGA-KOUMBA-BINZA \\ CENTRE for TeXt Technology (CTEXT) \\ NORTH-WEST UNIVERSITY, POTCHEFSTROOM CAMPUS
}

(22602560@nwu.ac.za)

\section{INTRODUCTION}

Le Gabon est certainement un pays à forte diversité linguistique comme le sont bon nombre de pays d'Afrique centrale. Les langues du Gabon ont plusieurs fois fait l'objet des classifications internes. Mais il faut noter que ces regroupements ont souvent été faits sur la base des critères linguistiques généralisés sur des langues n'ayant pas de norme standard.

En effet, de toutes les langues gabonaises, aucune n'est standardisée. Autrement dit, outre la question de leur introduction dans le système éducatif, l'un des défis majeurs de la linguistique gabonaise est la standardisation des langues.

De fait, les langues gabonaises, quel que soit le nombre qu'on peut leur donner, ne sont chacune qu'un ensemble de variantes. Une communauté linguistique telle que celle du ghisira ${ }^{1}$ qui paraît homogène est bel et bien subdivisée en variantes si l'on considère par exemple la distinction faite entre ghisira yi ngosi et ghisira yi kamba. Il n'est donc plus à démontrer que les langues du Gabon, même les plus modestes, nécessitent - si l'on peut parler ainsi - d'être standardisées, au vu de leurs diversités dialectales respectives. L'aspiration à la standardisation des langues du Gabon est manifeste par le fait que des études diverses (Emejulu et Nzang-Bié 1999b, Nzang-Bié 2002) ${ }^{2}$ ont été déjà entamées en vue de proposer un ou des modèles de standardisation.

L'objectif du présent article est de présenter une option de standardisation des langues du Gabon en se servant de l'identification des unités-langues et de leur classification interne. Pour ce faire, il convient d'observer préalablement les

1 Le ghisira est parlé dans la province du Moyen-Ogooué (région de Lambaréné) et dans la province de la Ngounié (région de Fougamou et Sindara).

2 Une recherche doctorale sur le dictionnaire bilingue comme instrument de standardisation du fang a été menée par Thierry Afane Otsaga. Nzang-Bié (2008) a par ailleurs publié un article sur un modèle standardisation $\mathrm{d}^{\prime}$ un dialecte fang. 
quelques réalités dialectologiques du Gabon. Puis, je présenterai les différents regroupements des langues du Gabon en unités-langues. Ceci conduira à une analyse de manière historico-dialectologique de la plus récente des classifications des unités-langues du Gabon. Nous pourrons enfin proposer une orientation de la standardisation des langues gabonaises.

\section{REALITES DIALECTOLOGIQUES DU GABON}

\section{CONCEPTS DE DIALECTE ET DE VARIETES DIALECTALES}

Bien que la dichotomie langue/dialecte ne soit pas facile à caractériser, celle-ci a déjà acquis une définition scientifique qui fait l'objet d'une certaine unanimité (Petyt 1980; Chambers \& Trudgill 1998). C'est-à-dire que le dialecte est tout simplement un système linguistique qui, dans un ensemble avec d'autres systèmes de même type, forme la langue. C'est ainsi qu'il est généralement dit que la langue est un ensemble de variantes dialectales.

Le terme de "dialecte" a le plus souvent été utilisé pour désigner une variante subalterne d'une langue. Dans une langue africaine bantu tel que le zulu par exemple, on distingue plus de six variantes régionales parmi lesquelles le tekela, le ntungwa et le zunda nguni (d'où est issue la forme standard). Il y a aussi le cas de l'anglais dont il est dit qu'il a plusieurs dialectes (Romaine 1994:900). On appelle dialecte régional la variété de langue associée à un lieu. C'est ainsi qu'on parle du dialecte anglais de Yorkshire en Angleterre et du dialecte allemand bavarois en Allemagne. Lorsque l'on voit dans la notion de dialecte un tel sens, il serait mal aisé d'évoquer des dialectes dans le cadre des langues gabonaises qui n'ont pas encore de forme standard. En d'autres termes, la notion de dialecte n'a de sens que par rapport à une forme standard de la langue.

De ce fait, la dialectologie au Gabon se pose comme dépendant du facteur de standardisation. Elle ne viendrait qu'une fois que des formes reconnues comme normes auraient émergé au sein et parmi les langues gabonaises respectives.

La notion de variété dialectale est généralement utilisée comme synonyme de dialecte ou variante dialectale. Définir la notion de variété dialectale reviendrait donc tout simplement à définir le terme de dialecte en soi-même. Je voudrais notifier ici que l'on peut se passer de cet amalgame terminologique en donnant une autre assertion au terme de variété dialectale. Dès lors, "variété dialectale" peut être également entendue comme la différence ou les différences qu'il y a d'un dialecte à un autre à un niveau donné du système linguistique. L'on peut ainsi observer des variétés dialectales grammaticales, phonologiques, lexicales, etc. Tandis que "dialecte" et "variante dialectale" (ou "régionale") restent quelque peu synonymes. 
Le terme de dialecte est diversement entendu par les chercheurs en linguistique et dialectologie. Pour Beaudoin (1998: 10), le dialecte n'est qu'une "variante régionale d'une langue; important pour l'identité de la communauté qui le parle". Ce qui fait qu'une autre expression synonyme donnée au terme de dialecte est variation régionale pour le distinguer de la variation sociale qui peut être plutôt un phénomène au sein d'une même variante linguistique; phénomène souvent dû à la subdivision de la société en classes, en groupes et en sous-groupes de toutes sortes (Petyt 1980 : 27-30). L'on pourrait à ce sujet citer l'exemple de la langue myenè (aussi appelée omyenè) qui se subdivise en variantes nommément appelées mpongwè, galwa, nkomi, orungu, adjumba et enenga. C'est ici une subdivision apparemment dialectale. Mais dans chaque subdivision se retrouvent des soussubdivisions qui sont quelque peu le résultat de la stratification sociale, puisque mpongwè, galwa, enenga, etc. constituant des sociétés différentes les unes des autres, rien que par le seul fait que chacune de ces communautés a une localisation particulièrement définie. Car, "les Mpongwe habitent les deux rives de l'Estuaire du Como; les Adyumba sont établis au Lac Azingo; les Galwa sont installés à Lambaréné et dans les grands lacs (Onangué, Ezanga, Ogouèmouè); les Orungu habitent le Cap Lopez et le Delta de l'Ogooué; les Nkomi vivent au Fernan-Vaz, entre le Cap Lopez et le Cap Sainte Catherine; les Enenga sont isolés au Lac Zilè" (Mouguiama-Daouda 1997: 168).

Petyt (1980: 11) pour sa part souligne que les dialectes sont simplement des formes différentes d'une même langue. Dans la langue fang par exemple, l'on note différentes formes même au sein d'une variante dialectale dite homogène. C'est ce que l'on observe dans le fang-ntumu parlée dans la province du Woleu-Ntem (Nord du Gabon). Dans ce fang-ntumu, l'on peut distinguer la variante parlée à Oyem de celle parlée à Bitam.

C'est fort des différentes formes que peut présenter une langue que Petyt (1980: 11) affirme que, "Using a language thus necessarily involves using one of its dialects". Ceci pour dire que parler une langue implique nécessairement faire usage d'un des dialectes de cette langue. L'on peut ainsi conclure que tout individu parle au moins un dialecte d'une langue, même si celui-ci serait caractérisé de langue standard (Chambers \& Trudgill 1998: 3).

On distingue la langue du dialecte en cela que deux locuteurs d'une même langue, mais de dialectes différents, peuvent se comprendre mutuellement alors que deux locuteurs de langues différentes ne peuvent pas se comprendre (Beaudoin 1998). Cependant la distinction entre dialectes repose sur le critère de la variation. En effet si deux locuteurs de dialectes différents sont intercompréhensibles, il n'en demeure pas moins que l'on distingue un locuteur d'un autre par le fait du parler qui varie de l'un à l'autre. Si cette variation se limite aux deux locuteurs sélectionnés, on parle d'un cas d'idiolectes; mais si la même variation va jusqu'à distinguer à partir des deux locuteurs deux groupes sociaux d'une même 
communauté linguistique, on parlera d'un cas de sociolectes ${ }^{3}$. Les idiolectes et les sociolectes peuvent être le résultat de la stratification sociale sus-évoquée. Par contre, cette variation n'est dialectale que dans la mesure où elle distingue à partir des mêmes deux locuteurs deux systèmes linguistiques mutuellement intelligibles.

La variation linguistique est vaste. En dépit du fait que chaque personne ait ses propres variations à l'intérieur d'une même langue, il y a des similarités importantes dans la façon de parler des membres d'une communauté donnée qui se distinguent des membres des autres communautés parlant la même langue. C'est là qu'on parle de variation dialectale ou régionale.

De même, l'usage fait du fang en Guinée Equatoriale en ce qu'il diffère de celui du Gabon est de fait une variation dialectale de cette langue comme le sont évidemment tous les autres usages. En d'autres termes, l'on dira que la langue fang a des variantes dialectales ${ }^{4}$ qui se trouvent en Guinée Equatoriale et au Gabon. On en trouve également au Congo (Brazzaville) et au Sao Tome et Principe (Grimes $\left.1996^{5}\right)$.

Tout en restant intercompréhensibles, deux dialectes d'une même langue entretiennent des différences qui permettent aux locuteurs de les démarquer des utilisateurs des autres dialectes. L'on note souvent que cet état de fait tient d'une part au besoin d'identité à un groupe spécifique par les individus qui le composent et d'autre part au développement naturel d'une langue qui évolue par son usage de façon différente dans différentes régions.

Ces différences qu'elles soient dues à l'évolution naturelle ou à la volonté de démarcation d'une communauté donnée sont dites variétés dialectales parce qu'elles contrastent différentes formes (appelées ici dialectes) d'une même langue. Les variétés dialectales, ou encore les différences entre dialectes, sont le fruit de la variation. C'est-à-dire les divers changements linguistiques qui se produisent lors de l'évolution normale (ou non) des langues en isolation et/ou en contacts interlinguistiques.

\section{TYPES DE VARIETES DIALECTALES}

Les variétés dialectales dans une langue sont de diverses sortes selon les différentes variations ayant été enregistrées (ou en cours) par la langue. Comme le disent Romaine (1994: 900) et Petyt (1980:16-17), un dialecte diffère des autres

3 Les variations des cas d'idiolectes et de sociolectes sont du domaine de la sociolinguistique. Ce qui n'est pas forcément le cas de la variation dialectale qui est plutôt l'objet de la dialectologie.

4 Dans cette exemplification je ne tiens pas compte des classifications (Hombert et autres) évoquées dans les pages qui vont suivre.

5 Grimes cite à ce sujet Johnstone (1993). 
dialectes de la même langue simultanément en au moins trois niveaux d'organisation: prononciation, grammaire ou syntaxe et vocabulaire.

Il s'agit plus précisément de relever comparativement les caractéristiques de différentes réalisations de la langue à l'étude sur les plans lexical, grammatical (syntaxe et morphologie), phonologique (morphophonologique y compris) et phonétique. Le cas de la dialectologie du fang dont je fais mention plus loin se base sur une comparaison lexicale et sur une comparaison phonologique et morphophonologique.

Des comparaisons à tous les niveaux d'organisation dans le cadre d'une étude dialectologique sont aisément applicables aux langues du Gabon. Elles seraient dans la plupart des cas amenées à distinguer des dialectes des uns des autres par le biais des différences que ceux-ci présenteront dans les divers niveaux sus-indiqués.

Dans le domaine de la langue yipunu par exemple l'on pourrait identifier selon des paramètres lexicaux des variantes suivantes de cette langue à partir des lexèmes qui désignent respectivement le bâton de manioc (maniot esculenta), la marmite et la négation. L'on pourrait distinguer le yipunu de Moabi/Ndéndé de celui de Mouila et de celui de Tchibanga6.

\begin{tabular}{|l|l|l|l|}
\hline Moabi/Ndéndé & Mouila & Tchibanga & Traduction \\
\hline mughuma & mulemba & ikwanga & "bâton de manioc" \\
\hline dwengu & mbidji & dwengu/mbidji & "marmite" \\
\hline nesi & viagha/nesi & nesi & "non" \\
\hline
\end{tabular}

Au niveau morphophonologique, le syntagme pour dire "j'ai dit que"7 nous donne dans la même langue yipunu les variantes Moabi/Ndéndé, Mouila et Tchibanga.

\begin{tabular}{|l|l|l|l|}
\hline Moabi/Ndéndé & Mouila & Tchibanga & Traduction \\
\hline nitsavabwendiri & nitsavayiri & ndzivayi/nitsavayi & "j'ai dit que" \\
\hline nitsavabwe & nitsavayinana & nitsavayina & "j'ai dit que" \\
\hline
\end{tabular}

La comparaison dialectologique soulignée plus haut peut être étendue à la grammaire, notamment au niveau de la morphologie verbale. L'on observerait des

6 Je rappelle qu'il s'agit ici d'une simple exemplification du processus d'identification dialectologique, et non pas une étude dialectologique en tant que telle qui nécessiterait un corpus non seulement plus large mais aussi plus élaboré.

7 A ne pas confondre avec le terme pour dire "je dis que" retenu comme critère de classification par Kwenzi-Mikala $(1988 ; 1998)$. 
variations dialectales du type qu'il y a entre le fang-ntumu et le fang-nzaman dans le syntagme pour dire "je ne suis pas là".

\begin{tabular}{|l|l|l|}
\hline fang-ntumu & fang-nzaman & Traduction \\
\hline maseki va & masara va & "je ne suis pas là" \\
\hline
\end{tabular}

L'extension de la comparaison à la grammaire aboutit à une comparaison globale, c'est-à-dire multidimensionnelle des systèmes.

La comparaison multidimensionnelle est de nos jours la procédure la plus objective qui requiert de la rigueur scientifique dans l'identification des dialectes d'une langue. Elle considère premièrement les éléments de variation au niveau du système phonologique (comme cela a été le cas du fang que j'évoque un peu plus loin), puis le lexique et la grammaire. La prononciation, ainsi que je l'ai évoqué précédemment constitue également une strate importante où s'opèrent des variétés dialectales.

Le paramètre le plus évident de différenciation dialectale au niveau de la prononciation est l'accent, c'est-à-dire le phénomène qui consiste en une façon particulière de prononcer d'une variante dialectale ou régionale d'une langue ${ }^{8}$. L'étude dialectologique multidimensionnelle est, en plus d'être objective, appropriée pour l'identification des divers dialectes et langues du Gabon. Elle pourrait également permettre de déterminer de manière objective le statut de chaque groupe linguistique.

\section{LA DIALECTOLOGIE AU GABON}

La dialectologie est une discipline toute aussi récente que la lexicographie au Gabon, tout comme l'est la linguistique en général. Au Département des Sciences du Langage (DSL) de l'Université Omar Bongo (UOB), à l'Institut de Recherche en Sciences Humaines (IRSH) du Centre National de la Recherche Scientifique et Technologique (CENAREST), tout comme au Laboratoire des Sciences Humaines et de la Dynamique du Langage (Lascidyl) de l'Ecole Normale Supérieure (ENS) et au Département des langues nationales de l'Institut Pédagogique National, la recherche est caractérisée par un intérêt moindre des études à caractères dialectologiques. Ainsi dans son Aperçu des travaux réalisés sur les langues gabonaises jusqu'en 2000, Idiata (2002: 45) recense seulement deux travaux au traitant des phénomènes dialectologiques dans quelques langues du Gabon.

8 Les faits de variétés dialectales au niveau de la prononciation sont l'objet d'une recherche en cours que mène H.S. Ndinga-Koumba-Binza. 
Il s'observe même une certaine confusion entre la dialectologie et la sociolinguistique au sein de la linguistique gabonaise. Cette confusion est vraisemblablement entretenue par l'insertion dans l'enseignement de sociolinguistique de la dialectologie comme parfois sujet des travaux dirigés ${ }^{9}$ au DSL. Cette confusion est également entrevue dans les programmes de recherche du Lascidyl qui, profondément ancré dans la sociolinguistique, ne s'empêche pas de mener des travaux de recherche à caractère dialectologique sans pouvoir préciser la distinction entre les deux disciplines ${ }^{10}$.

La dialectologie est généralement entendue comme l'étude des langues non standardisées ou non standard. Pour des auteurs comme Chambers et Trudgill (1998: 3) et Romaine (1994: 900), la dialectologie est de toute évidence l'étude de dialecte et des dialectes. Point n'est besoin de rappeler ici que cette définition se base sur la dichotomie désormais classique entre langue et dialecte. Dichotomie selon laquelle, on appelle langue, la variante standard et reconnue comme norme d'un système linguistique; et dialectes toutes les autres variantes de ce même système linguistique.

Définir la dialectologie sous cet angle stricto sensu amène inévitablement à la conclusion que cette discipline n'est pas encore pratiquée au Gabon selon les principes qui le lient à la standardisation préalable des langues. En effet, aucune langue gabonaise n'a une forme standard à partir de laquelle les autres formes devraient être considérées et étudiées comme dialectes. Sans norme, on ne saurait avoir de dialectologie. La dialectologie se trouve comme je le dis déjà plus haut, dépendante du facteur de standardisation.

Toutefois, lorsque l'on considère la dialectologie dans sa corrélation avec les notions de géolinguistique et d'atlas linguistique, elle porte tout un autre sens qui lui donne une existence au Gabon. Vue dans un sens large, la dialectologie a eu son entrée au Gabon avec le lancement par l'Etat Gabonais du Projet d'Atlas linguistique du Gabon à la fin des années 1980 (Dodo-Bounguendza 2001: 33). Ce projet est mené par les chercheurs du Laboratoire de Phonétique et Linguistique Africaine (LAPHOLIA) de l'Université Lumière de Lyon (France). Ainsi que le souligne Dodo-Bounguendza (2002: 33), ce projet "s'appuie sur le volet linguistique qui comprend l'inventaire des langues en usage au Gabon, la situation géographique de ces langues, la collecte des données minimales en vue de la classification de celles-ci".

9 Je fais allusion ici aux cours de Niveaux de langue et variétés de langue (1ère Année) et de Langue et saisie du monde dans la société (2è Année) qui étaient dispensés au Département des Sciences du Langage (Université Omar Bongo) depuis sa création en tant que Cour Magistral et Travaux Dirigés jusqu'à ce que les programmes soient révisés en 1999-2000.

10 Je citerai pour ce cas le séminaire de dialectologie organisé en décembre 1997 par le Lascidyl. Les travaux de ces assises n'ont pas manqué de soulever des perspectives sociolinguistiques. Cependant, pour des auteurs comme Petyt (1980 : 28-30) la sociolinguistique et la géolinguistique seraient en quelque sorte des composantes de la dialectologie. 
Malheureusement "la matérialisation objective des résultats obtenues pendant ces différentes périodes de terrain se font attendre" (Dodo-Bounguendza 2002: 33).

Les questions de l'inventaire des langues et de leur classification échoient de toute évidence et nécessairement à des études du type dialectologique, puisqu'elles impliquent inévitablement l'identification préalable des divers systèmes linguistiques de l'espace géographique en étude. Le chercheur aura à se préoccuper de distinguer entre les systèmes mutuellement intelligibles qui pourraient sensiblement être une même langue et les systèmes qui, bien que partageant le même environnement, ne sont pas intercompréhensibles; constituant ainsi des langues toutes différentes les unes des autres si elles ne sont pas de la même famille ou branche linguistique sur le plan historique.

Ainsi, les processus de classification et d'inventaire linguistiques participent pleinement à l'étude dialectologique en ce qu'ils requièrent l'observation de certains traits pertinents, à savoir les différences lexicales, morphophonologiques ou phonologiques et même phonétiques, des différents systèmes linguistiques d'une région en vue de leur identification et de leur catégorisation et/ou typologie. La dialectologie vue selon ce cheminement est celle qui a toujours eu cours au Gabon, depuis les enquêtes pour l'Atlas Linguistique du Gabon jusqu'aux travaux de Kwenzi-Mikala (1988; 1998) sur l'identification et la classification des parlers du Gabon en unités-langues.

Les quelques travaux purement dialectologiques publiés à ce jour sur les langues du Gabon sont ceux qui portent essentiellement sur le fang (Hombert 1991, MedjoMvé 1997a, 1997b et 1997c). Le fang demeure à ce jour la seule langue gabonaise ayant fait l'objet d'études dialectologiques au sens stricto sensu et dont les résultats sont concluants. Je mentionnerai ici les travaux de Hombert et de Medjo-Mvé dont le domaine de comparaison est le système phonologique.

L'étude de Hombert juge la traditionnelle répartition du fang en six sous-groupes (mekè, nzaman, ntumu, okak, mvaï et atsi) de classification impressionniste et linguistique. Pour Hombert, le système dialectologique du fang repose sur deux aires dialectales distinctes (Nord et Sud), en dépit de la difficulté d'accès des variantes de la Guinée Equatoriale dont le lexique lui a semblé assez bref. Cette classification de Hombert, illustrée par une carte géolinguistique, se base sur un lexique à critères morphophonologiques et sur une analyse des données collectées non seulement sur le terrain mais aussi à partir les travaux effectués sur la langue.

Medjo-Mvé (1997a) dans sa thèse sur la phonologie panchronique du fang esquisse une investigation sur les différents dialectes de cette langue en tant qu'elle est un moyen pour reprendre l'histoire linguistique et pourvoir aux manquements des reconstructions historiques et comparatives. Il confirme sur la base aussi bien des critères purement phonologiques que des paramètres lexicaux, les deux aires 
dialectales de Hombert (1991) et revoit d'une certaine manière la carte linguistique fang établie par Hombert. Dès lors, les six variantes du groupe linguistique fang composent diversement les deux zones dialectales identifiées de la manière représentée dans la figure qui suit.

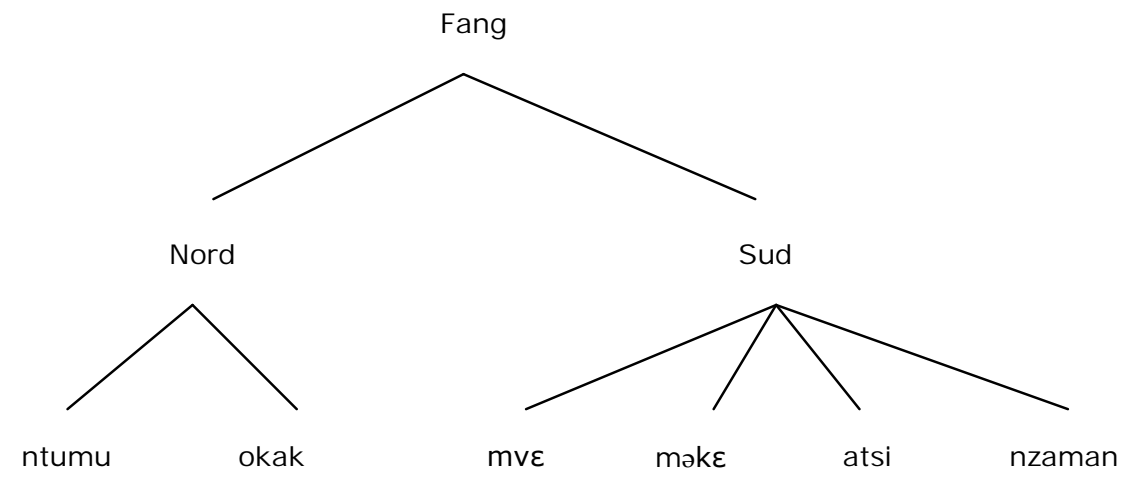

Figure 5: Composition dialectale du fang d'après Hombert (1991) et Medjo-Mvé (1997)

La dialectologie au Gabon s'arrête à ce travail minutieusement mené sur le plan scientifique et qui démontre au-delà des conceptions historico-sociologiques ${ }^{11}$ la situation dialectale d'une des langues du Gabon.

Comme déjà mentionné, les corrélats de la notion de dialectologie sont celles de géolinguistique et d'atlas linguistique. Certains auteurs à l'instar de Bothorel-Witz (2002) considèrent comme domaine de la géolinguistique dialectale les travaux à orientation sociolinguistique liés au contact de langues. "Ils portent, plus particulièrement, sur l'élaboration d'un modèle de variétés conçu comme un continuum de pratiques, sur la conscience psycho-sociolangagière des locuteurs dialectophones [...] sur les aspects (socio)linguistiques de l'évolution des dialectes actuels, sur les minorités et les phénomènes de la minoration".

A défaut d'être une discipline à part entière, la géolinguistique se définit selon Crosbie (1994: 1414) comme la théorie de la géographie dialectale ou encore de la linguistique cartographique. Pour lui, la géolinguistique consiste à présenter sur carte les caractéristiques linguistiques dans une zone géographique définie. Elle se confond ainsi avec l'atlas linguistique dont l'objet est de représenter la distribution des dialectes et langues d'une région donnée.

En dépit de cette situation peu effective, la dialectologie en tant que discipline linguistique a cependant un champ d'étude tout ouvert au vu de la diversité

11 On a en effet longtemps pensé que le domaine fang était homogène. 
linguistique du Gabon et du statut non encore concrètement spécifié ni identifié de ses langues et dialectes. Je pense qu'à ce jour, la dialectologie gabonaise s'est maladroitement orientée dans une perspective qui sous-entend l'étude de dialecte et des dialectes comme présenter plus haut. En effet, cette définition, quoique distinguant la langue du dialecte, s'investit dans le sens selon lequel on appellerait langue la variante (d'une langue donnée) considérée standardisée et dialectes toutes les autres variantes de cette même langue, comme je l'ai déjà indiqué. Les perspectives heureuses de la dialectologie gabonaise pourraient reposer premièrement sur l'orientation géolinguistique et atlas linguistiques, deuxièmement sur l'identification au sein d'un même système des variantes de ce système, et troisièmement sur le regroupement en unités-langues ou langues des divers systèmes linguistiques.

\section{L'IDENTIFICATION DES UNITES-LANGUES DU GABON}

Les langues du Gabon ont été l'objet de bon nombre de regroupements en unitéslangues. A la différence de Guthrie (1953) et de Jacquot (1985), Raponda-Walker $(1998)^{12}$ et Kwenzi-Mikala $(1988,1990,1998)$ présentent en plus de l'identification des unités-langues une classification interne de celles-ci.

Dans son ouvrage The Bantu languages of Western Equatorial Africa, Guthrie (1953) rassemble les idiomes bantu du Gabon en entités qui recouvrent la notion d'unitélangue. On a ainsi selon la classification de Guthrie les groupes suivants:

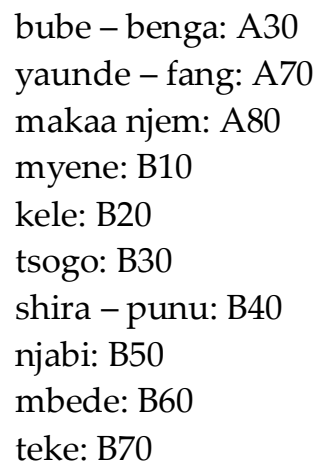

Cependant cette classification ne détermine pas le nombre des langues parlées au Gabon ni ne prend en compte des idiomes identifiés à l'heure actuelle sur le territoire gabonais. De plus, on y trouve aussi bien des langues parlées au Cameroun qu'en Guinée Equatoriale comme on le voit ci-dessus dans les groupes 1 et 2. Cette classification présente par conséquent des ressemblances avec la classification générale des langues bantu proposée par l'auteur lui-même. C'est au

12 Réédition de la Fondation Raponda Walker. 
sujet de cette dernière qu'Idiata (2002: 41) dit "l'ancrage diachronique adopté par Guthrie ne peut pas régler le problème de l'inventaire des langues vernaculaires gabonaises en synchronie".

S'appuyant sur les travaux de Guthrie et sur des recherches personnelles, Jacquot (1978) identifie dix groupes de langues gabonaises. Sa dixième unité-langue n'est rien d'autre qu'une classe unique contenant le civili qu'il considère sans aucune mutuelle intelligibilité avec les langues de la zone B. Cette classification se présente comme suit:

bube - benga: A30
ewondo: A70
myene: B10
kele: B20
tsogo: B30
shira: B40
njebi: B50
mbede: B60
teke: B70
civili: H10

Hombert (1990: 30) dit de l'inventaire de Jacquot qu'elle est une "présentation autour d'une classification linguistique (en reprenant et en modifiant la classification de Guthrie)". Comme celle de Guthrie, la classification de Jacquot a apparemment pour critère important celui de l'intercompréhension. Cependant il n'est pas fait mention des critères à partir desquels l'intercompréhension aurait été mesurée. Il est cependant connu que la classification générale des langues bantu de Guthrie repose non seulement sur le critère d'intercompréhension mais aussi sur ceux d'affinités grammaticales et d'affinités phonologiques. Cette classification de Jacquot présente les mêmes incorrections que celle de Guthrie, à savoir principalement la détermination inexacte des langues du Gabon et l'insuffisance du répertoire au regard du nombre des systèmes linguistiques observables à l'heure actuelle au Gabon.

Pour Raponda-Walker les idiomes ${ }^{13}$ gabonais se répartissent en six groupes linguistiques, eux-mêmes divisés en sous-groupes comme ci-dessous présenté.

Groupe I: Ngwe-myene

a)

mpongwè

galoa

nkomi

orungu b)

okandé

apindji

mitsogo

simba

13 Raponda-Walker utilise plus le terme idiome que celui de langue. 

adyumba
bavové
énénga
évia

Groupe II : Fang

bétsi

ntum

bule

ewondo

fong

nzamane

mékè

bakwélé

Groupe IV :

éshira

masango

bavarama

bavungu

bapunu

balumbu

ngowé

mindasa

basisihu
Groupe III:

$$
\begin{aligned}
& \text { bakélé } \\
& \text { bangomo } \\
& \text { bantomboli } \\
& \text { shaké } \\
& \text { dambomo } \\
& \text { mbanhu } \\
& \text { bawumpfu }
\end{aligned}
$$

Groupe V:

$\begin{array}{ll}\text { a) } & \text { b) } \\ \text { bénga } & \text { béséki } \\ \text { bapuku } & \text { balengi } \\ \text { kombè } & \\ \text { banoko } & \\ \text { batanga } & \\ \text { bakota } & \\ \text { manongwè } & \\ \text { bushamay } & \end{array}$

Groupe VI :

baduma

bavili

banzabi

bawandji

batsayi

mindumu

ambèdè

ambamba

atègè

akaninhi

Les critères seraient d'ordre historico-culturel et d'intercompréhension ou encore d'ordre ethnolinguistique, quoique l'auteur lui-même affirme qu'il s'agit d'une classification linguistique et non ethnique14. "Mais rappeler que la langue, la race, -nous ajoutons la culture- sont des paramètres qu'il faut considérer séparément est une chose, apporter des preuves de ce que l'on énonce en est une autre" (Mouguiama-Daouda 1997: 170).

14 Raponda-Walker utilise généralement le terme de "races" pour parler des ethnies. 
D'après Idiata (2002: 41), "la proposition de Raponda-Walker est trop ancienne pour espérer trancher le débat actuel sur le nombre des langues et leur distribution". De plus, bien d'autres idiomes tel que le civili (que l'auteur lui-même désigne baloango) ne figurent pas dans le répertoire de Raponda-Walker bien qu'il ait eu à en faire une étude des similitudes et divergences parmi les autres idiomes.

Kwenzi-Mikala $(1988 ; 1990,1998)$ dans sa classification interne des parlers du Gabon, regroupe les langues du Gabon en dix unités-langues:

MAZUNA: fang-atsi, fang-make, fang-mvaï, fang-ntumu, fangnzaman et fang-okak.

MYENE: enenga, galwa, mpongwe, nkomi, orungu et adjumba.

MEKANA-MENAA: akele, ungom, lisigu, mbangwe, metombolo, seki, tumbidi, shake, wumpfu et lendambomo.

MEKONA-MANGOTE: ikota, benga, shamayi, mahongwe, ndasa et bakola.

MEMBE (ou OKANDE-TSOGO): ghetsogho, ghepinzi, kande, ghevove, ghehimbaka, gheviya, ebongwe et kota-kota.

MERYE : ghisira, ghivarama, ghivungu, yipunu, yilumbu, yisangu, ngubi, civili, yirimba et yigama.

METYE: yinzebi, yitsengi, yiwele, yivili, liduma, liwanzi et yibongo.

MEMBERE: lembaama, lekaningi, lindumu, lateghe et latsitseghe.

MAKENA: bekwil, shiwa (ou makina) et mwesa.

BAKA: baka.

Ce regroupement est basé sur un critère sociologique, la formule de conversation «je dis que » ainsi que sur le critère d'une relation d'intercompréhension que les parlers d'une même unité-langue semblent entretenir. Il y est cependant omis les moyens par lesquels l'intercompréhension a été mesurée. Il faut toutefois souligner qu'Emejulu et Nzang-Bié (1999a, 1999b) reprochent à Kwenzi-Mikala de ne pas prendre position sur la question des dialectes. Auquel cas, le nombre de langues, dont Barbara Grimes (1996) suppose une quarantaine, pourrait être revu à la baisse. Selon Hombert (1990: 30) "Kwenzi-Mikala opte pour une présentation géographico-administrative".

Idiata (2002: 42), qui lui trouve un défaut de rigueur par le fait d'un seul critère, lui reconnaît cependant au moins deux avantages lorsqu'il dit: "c'est une classification récente et elle a l'air beaucoup plus détaillée et plus proche de la réalité de terrain". Je pense que de tous les inventaires et autres classifications observés ci-dessus le travail de Kwenzi-Mikala, en dépit des reproches sus-évoqués, présente l'avantage non seulement de tenir compte d'un maximum des parlers du Gabon, mais aussi de les regrouper en 10 unités-langues à l'intérieur desquelles les différents parlers entretiendraient plus ou moins des relations d'intercompréhension. Ce qui réduit 
considérablement le nombre de langues et permet de regrouper sous forme de dialectes les cas les plus évidents.

Aux dires d'Idiata (2002: 42) l'information sur le degré d'intercompréhension (entre les composants des unités-langues) qui manquent à l'argumentation de Kwenzi-Mikala "aurait été déterminante pour permettre de trancher l'épineux débat sur le nombre de langues vernaculaires au Gabon". En effet, il est nécessaire dans le regroupement sur la base du critère $d$ 'intercompréhension de mesurer le niveau d'intelligibilité entre les langues ou idiomes d'un même groupe. Ceci permet de distinguer de première vue les différents systèmes qui peuvent s'avérer être des dialectes d'une même langue.

La mesure du niveau d'intercompréhension permet également d'observer les langues les plus intelligibles entre elles et celles qui le sont moins. Néanmoins dans le cas particulier du Gabon où il s'agit de partir du regroupement en unitéslangues pour aboutir à des langues standard, il n'est plus besoin du degré d'intercompréhension si l'on part d'une approche historico-dialectologique de chaque unité-langue.

\section{DES UNITES-LANGUES AUX LANGUES}

D'après Kwenzi-Mikala (1988: 57; 1990: 122) "Une unité-langue est l'ensemble de différents parlers tous mutuellement compréhensibles". Kwenzi-Mikala (1988: 58) souligne que pour l'établissement de sa classification "la première étape du travail de terrain a consisté à obtenir des villageois une information générale sur la situation linguistique telle qu'elle était perçue par eux-mêmes". Cette étape pour laquelle des enquêtes ont été menées dans les villes aussi bien que dans les villages, précise l'auteur, fut précédée par une consultation des travaux de Guthrie (1953) et d' Jacquot (1978) qui donnent un aperçu sur la répartition géographique des groupes linguistiques du Gabon et leur classification interne, comme présenté précédemment.

La seconde étape de cette recherche "a été l'analyse de la situation sociolinguistique par le questionnaire sur l'intercompréhension. Cette seconde phase du travail a consisté à demander aux informateurs de parlers différents s'ils pouvaient comprendre sans apprentissage ni utilisation d'une tierce langue à la première rencontre, s'ils dénombraient une ou plusieurs langues et s'ils pouvaient donner le ou les noms de ces langues".

Selon Kwenzi-Mikala (1988; 1998), il ressort de cette enquête qu'il y a dix groupes de parlers au Gabon ${ }^{15}$ ainsi présenté plus haut. Cette classification a aussi le mérite

15 Je précise qu'en 1988 l'auteur énumère huit groupes. Dix ans plus tard, il révise son travail et porte le nombre des unités-langues finalement à dix. Je présente ici la version révisée et officiellement 
non seulement de répertorier et d'identifier les systèmes linguistiques du Gabon connus jusqu'à ce jour, mais également de les rapprocher selon leurs affinités, c'està-dire en fonction de leur mutuelle compréhension et de leur lexique verbale (si l'on parler ainsi du terme pour dire "je dis que"). D'après Kwenzi-Mikala (1988: 59) "Le dépouillement du questionnaire a permis de montrer que, contrairement à l'idée reçue qui veut qu'il y ait quarante langues, le nombre d'unités-langues est réduit. En effet, si l'on procède au regroupement des parlers sur la base de l'intercompréhension, on obtient huit [aujourd'hui dix] langues... Etant donné que ces langues n'ont pas toutes de nom spécifique, j'ai dî avoir recours au terme qui sert à engager une conversation et qui équivaut à 'je dis que' ».

Je précise que pour certains linguistes gabonais le travail de Kwenzi-Mikala n'est pas une classification mais plutôt un inventaire pour la simple raison qu'une classification repose sur des critères génétiques (cf. l'Indo-européen avec les auteurs de la linguistique comparée ou la classification des langues bantu de Guthrie). Mon point de vue est que le travail de Kwenzi-Mikala est un inventaire des unités-langues, mais c'est une classification à l'intérieur de chaque unitélangue. C'est une prise de position quelque peu politique et non strictement scientifique; et j'use du terme de classification pour les besoins que j'ai l'identifier.

En dépit de quelques incorrections à l'instar de celle de l'intercompréhension entre le civili et les autres parlers du groupe MERYE (Ndinga-Koumba-Binza 2000: 1113; Kwenzi-Mikala 1988: 59; Jacquot 1985: 355-359), ce travail de Kwenzi-Mikala est reconnue par la majorité des chercheurs sur les langues gabonaises comme une source de référence vue son ambition de réduction du nombre des langues du Gabon. En effet, les politiques linguistiques et l'enseignement des langues nationales $\mathrm{du}$ Gabon ne pourraient pas se passer de la connaissance du nombre exacte ainsi que de l'inventaire de ces langues. Comment peut-on vouloir promouvoir et enseigner des langues dont on ne connaît ni le nombre exact ni les différentes variantes? En proposant une identification des unités-langues du Gabon et leur classification interne, Kwenzi-Mikala (1988) a anticipé à l'époque la réponse à cette question.

L'on sait aussi que "L'identification des unités-langues bantu gabonaises et leur classification interne" furent motivées, selon Kwenzi-Mikala (1988: 55), "par le fait qu'il est communément admis qu'il y a quarante langues au Gabon". Dans le cadre d'une approche nouvelle et adéquate des problèmes de classification linguistique au Gabon, l'inventaire de Kwenzi-Mikala mériterait d'être revisité non plus sous l'optique d'un regroupement des parlers en unités-langues, mais plutôt, et désormais, selon l'orientation d'une identification nette des langues à partir d'une approche historico-dialectologique.

reconnue lors de la 1ère Table Ronde sur les "Recherches Linguistiques et l'Enseignement des Langues au Gabon" du 9 au 11 décembre 1997. 
Il faut en effet noter que le terme de dialecte a aussi des connotations historiques. En linguistique historique, par exemple, on parle des dialectes germaniques en référence aux ancêtres des variétés de langue de nos jours reconnues comme langues germaniques modernes. Tel est le cas de l'anglais, du hollandais et de l'allemand pour ne citer que ces langues-là. Ce sont des dialectes du germanique qui ont fini par émerger comme langues selon des facteurs historiques, politiques et culturels (Romaine 1994: 900). De la même manière et selon des facteurs identiques les anciens dialectes du latin de l'Empire Romain ont émergé en langues française, espagnole, italienne et roumaine (Petyt 1980 : 32).

Pour revenir à la situation spécifique des langues du Gabon, le problème est de procéder à l'identification exacte de celles-ci et ce en s'appuyant sur le travail de Kwenzi-Mikala. Kwenzi-Mikala (1990: 122) lui-même reconnaît implicitement une synonymie entre les termes de langue et de groupe de parlers et d'unité-langue qu'il utilise lorsqu'il écrit: "la proposition d'identification des langues ou groupes de parlers que j'ai établie". Ceci revient à dire que Kwenzi-Mikala entend par langue ce qu'il désigne par unité-langue, et entend par dialecte ce qu'il désigne par parler ou sous-groupe d'unité. Je ne doute pas que Kwenzi-Mikala ait préféré les termes d'unité-langue et de parler pour s'éviter quelque débat ou réflexion sur le plan terminologique; les approches définitoires des notions de langues et de dialectes étant d'une sensibilité aiguë selon les perspectives linguistiques, sociologiques, historiques, culturelles et même politiques.

Cette perspective qui envisage les unités-langues en termes de langues et les parlers en termes de dialectes est clairement perceptible dans les groupes quasiment homogènes qui sont le MAZUNA ${ }^{16}$ et le MYENE. En effet, quoique les natifs des deux groupes manifestent leur appartenance à une variante donnée (atsi, okak, ntumu, nzaman, mvaï et mekè pour le fang; orungu, mpongwè, galwa, nkomi, adjumba et enenga pour le myenè), il n'en demeure pas moins que tous parlent la même langue. Cette détermination peut trouver une explication dans l'organisation interne des différents groupes ethnolinguistiques. "Dans le groupe B10 (Myene), tout comme dans le sous-groupe A75 (Fang), il s'agit sur le plan linguistique d'une seule langue avec ses dialectes et sur le plan ethnologique de plusieurs ethnies parlant une même langue" (Ndinga-Koumba-Binza 2000: 15). Nzaman, atsi, okak, mvaï, mekè et ntumu parlent tous le fang. De même, enenga, nkomi, galwa, mpongwè, orungu et adjumba parlent tous l'omyenè.

Pour ce qui est des cas des groupes hétérogènes et hétéroclites comme le MEKANA-MENAA, le MEKONA-MAךGOTE, le MEMBERE ou le MERYE, ils nécessiteraient quelques réaménagements. On pourra cependant trouver dans certains cas qu'il s'agirait des dialectes d'une même langue dont l'évolution tout à

16 Il a été présenté plus haut par Hombert (1991) et Medjo-Mvé (1997a, 1997b et 1997c) que le groupe fang n'est pas aussi homogène qu'on l'a toujours cru. 
fait rapide influence grandement l'intercompréhension entre les différents systèmes linguistiques. Le type d'évolution qu'il y a eu entre l'espagnol, le portugais et l'italien à partir du latin.

L'on notera aussi et ce d'une manière générale que dans d'autres groupes tels que le MAKENA ou le MEMBE il se présente une tacite organisation déterminative des variantes à l'image de celle du fang et de l'omyenè. Les natifs des différentes communautés de ces groupes se définissent généralement comme appartenant à une même et unique souche historico-ethnolinguistique partageant traditions, rites et cultures et origines. Il est donc vraisemblable que dans le cas des groupes MAKENA et MEMBE, on ait affaire à des dialectes d'une même langue ayant connu une évolution particulièrement rapide, celle-ci n'influençant que de façon minime l'intercompréhension entre les divers systèmes linguistiques. Le type d'évolution qu'il y a eu entre l'allemand, le hollandais et l'afrikaans à partir du germanique.

Il est nécessaire de noter que le regroupement des idiomes du Gabon présente des avantages divers telles que:

- la réduction du nombre des langues;

- la décomplexification du processus de standardisation des langues;

- et l'accessibilité du choix des langues à standardiser, officialiser et à insérer dans l'enseignement.

Ce regroupement implique la dialectalisation des différents sous-groupes. La notion de dialectalisation, quoiqu'elle produise d'un point de vue une divergence, peut être vue d'un autre point de vue comme phénomène de convergence (Petyt 1980: 32). Des communautés voisines et proches sur le plan linguistique qui seraient dans un processus de séparation (tel est le cas du makina qui, dans le groupe MAKENA, se sépare de plus en plus des mwesa et bekwil par un certain phénomène d'assimilation au groupe fang auront à retrouver une sorte d'unité dans une forme commune par le besoin que les individus d'un même groupe auront de communiquer régulièrement.

\section{STANDARDISATION DES LANGUES}

\section{GENERALITES}

La standardisation, au sens où je l'entends, est non seulement le développement naturel d'une langue standard dans une communauté linguistique, mais aussi l'imposition par un groupe d'un dialecte comme langue standard. Cette imposition peut être tacite ou implicite comme elle peut être par décision délibérément prise. Il existe bon nombre de modèles de standardisation. Mais d'une manière générale 
la standardisation de certaines langues du monde s'est faite suite à des facteurs extralinguistiques. Romaine (1994: 900) dit d'ailleurs que le processus de standardisation est lié à plusieurs facteurs sociohistoriques parmi lesquels on cite l'alphabétisation, le mouvement nationaliste, et l'identité culturelle et/ou ethnique.

En Grèce antique par exemple, chaque ville utilisait anciennement son propre dialecte à la fois pour la forme orale et pour la forme écrite jusqu'à ce qu'un système de communication plus large conduisant au dialecte d'Athènes fut adopté comme dialecte commun. Les raisons de ce choix, ainsi que l'indique Petyt (1980: 32-33), furent d'ordre social, économique et politique. Car le dialecte choisi fut tout simplement le parler de la classe sociale supérieure de la capitale de la nation, une classe qui inspirait déjà un certain prestige à tous les niveaux de la société, et particulièrement au niveau culturel par le biais des sciences, des lettres et des arts.

Le cas de la langue française est également un fait de standardisation conduite primordialement par des éléments extralinguistiques. Le statut officiel du français parmi toutes les autres langues du royaume de France (de l'époque) fut premièrement déclaré par l'Edit de Villers Cotterêts en 1539, et fortement réaffirmé, comme symbole de l'unité nationale et de la culture, par la convention de 1794 issue de la Révolution de 1789. Pour Prosner (1994: 1300), le français moderne qui triomphe dès le XVIè siècle est le résultat des efforts minutieusement fournis pour la codification et la standardisation de la langue et pour l'extension de son domaine.

Cependant, d'après l'auteur, ceci n'a pas empêché qu'au XVII siècle l'usage de la cour royale fut adopté comme la norme de la langue française, et avec quelques modifications, finalement imposé comme langue nationale et du système éducatif. Le scénario est quasiment le même quand Shaka, roi des Zulu, décida que ses sujets pouvaient parler n'importe quel dialecte de l'isiZulu à domicile, mais que dans son armée chaque guerrier était tenu de parler le dialecte du roi. Poulos \& Msimang (1998: 19) écrivent que Shaka utilisa ce facteur linguistique pour inculquer l'unité et la solidarité dans son armée, puis fit de celle-ci une institution prestigieuse et enviable dans tout le territoire par le biais de la promotion, des récompenses et autres faveurs à tout guerrier qui non seulement se distinguerait dans le champ de bataille mais qui parlerait en plus le bon Zulu.

Aussitôt, bon nombre de dialectes du Zulu ne furent plus parlés que par les femmes et les enfants. De nos jours, le Zulu standard parlé, écrit et enseigné dans les universités sud-africaines est dû au prodigieux dialectologue et sociolinguiste Shaka qui usa non pas de la coercition mais de la persuasion pour imposer son dialecte à lui comme norme du Zulu.

Dans toute langue, cependant, la forme retenue standard n'acquiert son statut de norme que par des chemins lexicographiques. C'est pourquoi Romaine (1994: 900) 
définit la standardisation comme résultant d'un choix et d'une fixation comme norme uniforme d'un usage particulier qui est promu et véhiculé au travers des dictionnaires, des manuels de grammaire et d'enseignement. Une langue standard est une variante qui a été délibérément codifiée de telle sorte qu'elle varie minimalement dans sa forme linguistique mais maximalement élaborée dans sa fonction, ajoute-t-elle.

En effet, le dictionnaire comme tout autre ouvrage didactique joue un rôle très important dans la standardisation d'une langue, celui de véhiculer la norme par l'autorité qu'il exerce auprès de ses usagers dans la société. Je dirais même que la lexicographie au travers du dictionnaire et de manuels d'enseignement fixe la norme de la langue par son influence sur le développement et la standardisation d'une langue. Il est effectivement notable que le dictionnaire participe à grande échelle à l'élévation au-dessus des autres d'une variante linguistique. La standardisation des langues gabonaises a donc nécessairement besoin de la lexicographie dans son sens le plus étendu.

Mabika Mbokou (2001: 220) cite, par exemple, le cas de l'afrikaans où "dans la lutte pour une reconnaissance comme langue officielle au même titre que l'anglais" la confection des dictionnaires s'était assignée l'objectif de la "standardisation par le développement et la promotion de la langue". Pour le cas du Gabon, la confection des dictionnaires dits descriptifs standard dont le but est de refléter la norme s'impose. Comme le précise Nyangone Assam (2001: 193), de tels dictionnaires s'orientent "généralement sur le présent et le futur" et ayant "un plus fort caractère normatif qu'un dictionnaire compréhensif". Pour le cas précis des langues gabonaises, la standardisation ne saurait pas se démarquer complètement d'un support du type dictionnairique ou lexicographique pour diffuser ou véhiculer la norme.

\section{LE CAS PARTICULIER DU GABON}

Dans la lecture que j'ai faite de la classification en unités-langues de KwenziMikala, j'ai présenté dans la section qui précède que l'on pourrait voir en chaque unité-langue une langue au sens proprement dit en tant que "phénomène social dont les règles de fonctionnement sont liées à d'autres règles sociologiques d'interactions humaines" (Kwenzi-Mikala 1988: 55). Et j'ajouterais à ces règles sociologiques aussi bien des règles historiques que des règles culturelles. Je perçois également en chaque sous-groupe d'unité-langue une variante dialectale de la langue anciennement considérée comme unité-langue.

Pour Emejulu et Nzang-Bié (1999a: 7), la classification des langues gabonaises de Kwenzi-Mikala semble offrir une plateforme théorique facile pour l'identification des langues à standardiser. Les auteurs pensent que l'avantage inhérent de cette classification est qu'elle donne aux planificateurs un modèle plus simplifié pour le choix d'une langue. Mieux, je trouve qu'elle oriente le processus de standardisation 
des langues du Gabon. A partir de cette classification de Kwenzi-Mikala, ce processus commence par reconnaître au lieu d'une quarantaine ou soixantaine, une dizaine de langues bantu parlées au Gabon.

La standardisation des langues consisterait donc au choix de l'un des dialectes anciennement sous-groupes d'une même unité-langue. Dans une langue comme le MAZUNA qui présente six formes dialectales, le processus de standardisation aurait à choisir entre l'atsi, le ntumu, le nzaman, le mekè, l'okak et le mvaï; dans le MYENE le choix serait entre le mpongwè, l'enenga, le galwa, le nkomi, l'adjumba et l'orungu; dans le MAKENA l'on choisirait entre le bekwil, le shiwa (ou makina) et le mwesa; etc.

Cependant il se poserait deux problèmes celui du facteur de standardisation et celui des critères de choix de la forme standard. Si le second cas présente une solution rapide et efficace, il n'en est pas de même pour le premier. Emejulu et Nzang (1999b) ont proposé quelques critères pour le choix d'un dialecte dans le cadre de la standardisation. Premièrement ils admettent les 18 critères énumérés par Sadembouo (1980) ${ }^{17}$ pour la sélection d'un dialecte standard, à savoir:

\section{CRITERES FONDAMENTAUX}

- le haut degré de compréhension déclarée du dialecte;

- le haut degré de compréhension prédite du dialecte;

- l'importance numérique des locuteurs du dialecte;

- la position géographique avantageuse du dialecte;

- la localisation du dialecte au centre d'activité;

- le prestige du dialecte;

- la pureté du dialecte;

- la véhicularité du dialecte.

\section{CRITERES SECONDAIRES}

- l'attitude du gouvernement envers le dialecte;

- l'influence religieuse du dialecte;

- l'importance socio-économique du dialecte;

- les documents écrits déjà existants dans le dialecte;

- l'expansion historique de la langue;

- le sentiment exprimé sur la facilité à comprendre et parler le dialecte.

17 Cité par les auteurs. 


\section{CRITERES MARGINAUX}

- la disponibilité des locuteurs du dialecte à coopérer dans le travail de développement de la langue;

- des bonnes conditions de travail pour les chercheurs;

- des relations d'amitié entre les chercheurs et un locuteur du dialecte;

- le statut social des locuteurs du dialecte.

A ceux-ci Emejulu et Nzang (1999b: 5) ajoutent les critères suivants à partir des sources diverses.

- des langues nationales d'une plus large communication;

- la décentralisation des décisions du choix des langues aux régions;

- des langues régionales dominantes;

- le choix laissé aux communautés;

- la langue native de la Capitale

- la présence des leaders engagés dans le processus de standardisation;

- la présence d'une communauté concernée par la standardisation;

- les langues en voie de disparition.

Il est évident, comme le reconnaissent les auteurs eux-mêmes, que le critère le plus important et le plus fonctionnel est l'acceptabilité des communautés linguistiques. Emejulu et Nzang (1999: 5) citent Sadembouo, Tadadjeu et Wiesman (1988) qui affirment que la décision est des hommes et non des principes, "People decide, not principles".

C'est fort de cette raison que je pense que le processus de standardisation des langues du Gabon aura du mal à se poursuivre avec uniquement des facteurs linguistiques et scientifiques. A mon avis, la standardisation des langues au Gabon au même titre que le choix d'un dialecte comme norme dans les différentes langues nécessite une décision politique qui intègre le mouvement de planification et d'aménagement linguistique déjà initié aussi bien par le Ministère de l'Education Nationale que par le GRELACO ${ }^{18}$ au sein de l'Université Omar Bongo. Car, il faut le mentionner, la situation spécifique des langues du Gabon ne cadre pas de manière adéquate avec la critériologie exposée plus haut.

Je proposerais donc une certaine coopération entre les chercheurs et les politiques dans le processus de standardisation de nos langues. Cette coopération est ici une solution au problème de facteur de standardisation, c'est-à-dire qu'elle va constituer l'élément moteur qui devra favoriser la standardisation de nos langues. Elle revient également à combiner les facteurs extralinguistiques et la recherche linguistique. Je voudrais noter que des expériences précédentes, telle que celle de

18 Groupe de Recherche en Langues et Cultures Orales. 
l'alphabet scientifique des langues du Gabon (ASG) ${ }^{19}$, ont échoué faute de cette coopération importante. Nzang-Bié (2001: 27) évoque la nécessité de cette coopération en ces termes, "Nous sommes conscientes que la question de la planification linguistique est politique et académique..."

En effet, quoiqu'il revienne au linguiste de concevoir les méthodes et autres processus d'enseignement ou de développement des langues, il ne lui revient malheureusement pas de décider de l'effectivité de cet enseignement ou de l'application effective de méthodes élaborées. Ceci revient au politique. Ce qui est également difficile ou impossible au décideur politique devra revenir au linguiste. Il faut également noter qu'après cinq décennies d'indépendance et l'enracinement du français dans les mentalités et traditions des Gabonais, le Gabon n'est plus au stade où des facteurs extralinguistiques comme ceux évoqués tout au long de ce texte - nationalisme, prestige culturel, etc. - peuvent effectivement participer à la standardisation des langues. Le seul facteur extralinguistique qui s'impose est celui de la décision politique. Mais celle-ci devra prendre en compte les résultats ou les propositions de la recherche linguistique.

En ce qui me concerne, je crois en une standardisation des langues du Gabon qui commence par la reconnaissance des unités-langues en langues et du choix quasi arbitraire (puisque les critères semblent inadéquats) d'un des dialectes comme norme dans chaque langue. Cette reconnaissance et ce choix devront s'inscrire dans une coopération entre chercheurs et décideurs. L'on pourra enfin introduire les normes reconnues dans le système éducatif que soutiendra la confection des dictionnaires et autres ouvrages d'enseignement.

En effet, on a souvent parlé de l'insertion dans le système éducatif des langues nationales. Cependant, qui dit enseignement d'une langue dit également norme. Puisque l'on n'enseigne pas une langue, mais la norme de cette langue. Le cas spécifique du Gabon suggère que l'enseignement des langues est un atout pour la confirmation et l'expansion de la norme admise dans chaque langue.

\section{DES CAS DE STANDARDISATION IMPLICITE}

Une certaine standardisation a commencé sans dire son nom dans des initiatives privées et par l'expérience des media publics et privés.

La première est celle de l'organisation non gouvernementale Ayile. Cette association dont la mission est la promotion et la sauvegarde de l'identité culturelle et linguistique myenè a en 1992 "édité un manuel élaboré et un coffret de cassette audio sur la méthode de l'enseignement d'un dialecte omyene intitulé: le mpongwe en 26 leçons"

19 Certains auteurs, à l'instar de P.A. Mavoungou (communication personnelle), pensent que l'ASG n'a pas échoué mais qu'il n'a pas reçu la publicité adéquate à sa vulgarisation. 
(Nzang-Bié 2001: 21). Comme précisé dans le titre du manuel, la variante myenè véhiculé est le mpongwè. Il est donc implicitement présenté dans le manuel d'Ayele que la norme myenè est le mpongwè. Les apprenants qui n'ont pas d'autres soucis que celui d'apprendre la langue myenè (et non le dialecte mpongwè) acquièrent ainsi comme norme, une des variantes de la langue qu'ils sont sensés apprendre et parler plus tard.

Le principe est quasiment le même dans la méthode Rapidolangue de la Fondation Raponda Walker. Dans le but de la promotion des langues et cultures du Gabon, ce groupe (composé des chercheurs, pédagogues et missionnaires) poursuit depuis 1995 l'expérimentation d'un manuel multilingue d'enseignement des langues gabonaises (Hubert 1995 \& 1997). Les deux premiers volumes de Rapidolangue actuellement expérimentés dans bon nombre d'établissements secondaires comprennent le fang, le yinzebi, le lembaama, l'omyenè, le yipunu, l'ikota, le civili et le ghetsogho 20 .

Pour les cas du fang et de l'omyenè, il est clair qu'il s'agit de la diffusion de l'un des dialectes respectifs précis du MAZUNA et du MYENE comme présenté dans la classification de Kwenzi-Mikala. Les cas du lembaama, du yinzebi, du yipunu, de l'ikota et du ghetsogho sont à l'évidence une situation du choix quasi arbitraire (dont j'ai déjà fait mention) parmi les langues MEMBERE (pour le lembaama), METYE (pour le yinzebi), MERYE (pour le yipunu et le civili), MEKONA (pour l'ikota) et MEMBE (pour le ghetsogho). Le lembaama, le yinzebi, le yipunu, l'ikota et le ghetsogho sont implicitement véhiculés comme norme de chaque groupe respectif. La situation du civili ici est telle qu'elle se présente dans la classification de Jacquot que nous avons vue plus haut : une classe unique.

En dehors de cette situation d'enseignement, la standardisation implicite est en outre observée dans les mass media publics et privés. A Libreville, la Capitale, comme au niveau des provinces, les stations de Radio et Télévision diffusent de plus en plus non seulement des émissions à caractères promotionnelles, mais surtout des informations d'actualités et des avis et communiqués en langues locales. Ce fait participe également à la standardisation implicite, puisque les langues qui passent à la télévision comme à la radio proviennent à quelques exceptions près de chaque groupe identifié dans la classification de KwenziMikala. Seuls les groupes MERYE (avec le yipunu et le ghisira), METYE (avec le yinzebi et le liduma) et MEMBERE (avec le lembaama et le latege) dont les diffusions sont faites dans plus d'une variante de ces langues.

La standardisation telle que je la perçois à partir de la classification de KwenziMikala consisterait donc en partie à planifier, réguler et officialiser ce phénomène

20 Voir l'article de Jacques Hubert, dans ce volume, pour des détails sur l'expérience de la Fondation Raponda Walker. 
que j'appelle standardisation implicite observable dans les media et les initiatives privées d'enseignement.

\section{CONCLUSION}

La standardisation des langues est un processus difficile à initier pour des pays comme le Gabon qui, après une cinquantaine d'années d'indépendance, s'est accoutumé à la langue héritée de l'ancienne puissance coloniale, reconnue comme unique langue officielle. Cette officialisation de la langue étrangère et cette accoutumance ont durablement fait passer les langues locales pour des laisséspour-compte dans les politiques et planifications linguistiques et les systèmes d'enseignement. Au moment où le Gabon se donne le souci de ses langues locales par la volonté de les promouvoir et les insérer dans le système éducatif, il se pose la difficulté de la non standardisation de celles-ci. Le présent article, après avoir esquissé la situation dialectologique du Gabon et interprété le regroupement des idiomes du Gabon en unités-langues, propose un certain point de départ pour la standardisation des langues du Gabon.

Il y est mentionné qu'en partant de la classification de Kwenzi-Mikala et par une coopération entre chercheurs et politiques, l'on aura affaire à dix langues seulement à standardiser en choisissant quasi arbitrairement dans chaque groupe un dialecte représentatif comme norme standard de la langue. Ceci ne ferait que réorganiser une certaine standardisation observée par ailleurs dans les media et les expériences privées d'enseignement des langues locales. Le choix quasi arbitraire remplace la critériologie élaborée et à laquelle le cas particulier de la situation gabonaise entre difficilement. Le modèle de standardisation trouvera un support d'expansion et de diffusion dans la confection des dictionnaires et autres manuels d'enseignement. Ainsi le processus de standardisation des langues se pose comme une des tâches importantes pour la lexicographie gabonaise en plein essor.

\section{RÉFÉRENCES}

Afane Otsaga, T. 2004. The standard translation dictionary as an instrument in the standardization of Fang. Stellenbosch: Stellenbosch University. Thèse de doctorat.

Ayile. 1992. Méthode d'apprentissage de la langue omyene. Libreville.

Beaudoin, M. 1998. Lexique de la linguistique. Dictionnaire disponible sur le site http://www.ualbeta.ca/beaudoin/ling/lexique

Bothorel-Witz, A. 2002. Domaine de la géolinguistique dialectale. Disponible sur le site web http://www.teluq.uquebec.ca

Chambers, J.K. and P Trudgill. Dialectology. Cambridge Textbooks in Linguistics. Cambridge University Press, 2è Edition. 
Crosbie, J.A. 1994. Geography and Language. R.E. Asher (Ed.). The Encyclopedia of Language and Linguistics, vol. 3: 1411-1417. Pergamon Press.

Dodo-Bounguendza, E. 2002. Les langues du Gabon, le point en 2001. Le Gabon et l'Unesco, Revue d'information. N²: 33-39. Libreville: Commission Nationale Gabonaise pour l'Unesco.

Emejulu, J.D. \& Y. Nzang-Bié. 1999a. Linguistic perspectives in Gabon. Colloque international. Grand Forks: University of North Dakota-SIL, Inc.

Emejulu, J.D. \& Y. Nzang-Bié. 1999b. Initial language development. Explaining Multilingual Education: Information on Some Tough Questions. 39-65.

Grimes, B. 1996. Ethnologue. $13^{\text {th }}$ Ed. Dallas: Summer Institute of Linguistics, Inc.

Guthrie, M. 1953. The Bantu languages of western equatorial Africa. Oxford University: Press for International African Institute.

Hombert, J.M. 1990. Les langues du Gabon: état de connaissance. Revue Gabonaise des Sciences de l'Homme, $N^{\circ}$ 2: 29-36. Libreville: LUTO-UOB.

Hombert, J.M. 1991. Quelques critères de classification des parlers fang. Pholia 6: 145-153. CNRS. Université Lumière-Lyon2.

Hubert, J. 1995. Rapidolangue: Méthode d'apprentissage des langues nationales. Volume N¹ (fang, inzebi, lembaama, omyene, yipunu). Libreville: Editions Raponda-Walker.

Hubert, J. 1997. Rapidolangue: Méthode d'apprentissage des langues nationales. Volume $\mathrm{N}^{\circ} 2$ (ikota, ghetsogho). Libreville: Editions Raponda-Walker.

Idiata, F.D. 2002. Il était une fois les langues gabonaises. Libreville: Editions Raponda-Walker.

Jacquot, A. 1978. Le Gabon. Daniel Barreteau (dir.), CILF: Inventaire des études linguistiques sur les d'Afrique noire d'expression française et sur Madagascar. Paris: SELAF.

Jacquot, A. 1985. Cahier ORSTOM, série Sciences Humaines, vol. XXI, N²-3.

Kwenzi-Mikala, J.T. 1988. L'identification des unités-langues bantu gabonaises et leur classification interne. MUNTU 8: 54-64. Libreville/Paris: CICIBA/Présence Africaine.

Kwenzi-Mikala, J.T. 1990. Quel avenir pour les langues gabonaises? Revue Gabonaise des Sciences de l'Homme, $N^{\circ}$ 2: 121-124. Libreville: LUTO/Publications de l’Université Omar Bongo.

Kwenzi-Mikala, J.T. 1998. Parlers du Gabon: classification du 11.12.97. A. Raponda-Walker, Les langues $d u$ Gabon. Libreville: Editions Raponda-Walker.

Mabika Mbokou, L. 2001. Le rôle du dictionnaire dans le système éducatif. J.D. Emejulu (éd.), Eléments de Lexicographie Gabonaise. Tome 1: 206-222. New York: JimacsHillman Publishers.

Medjo-Mvé, P. 1997a. Essai sur la phonologie panchronique des parlers fang du Gabon et ses implications historiques. Thèse de Doctorat (NR). Université Lumière-Lyon2.

Medjo-Mvé, P. 1997b. Dialectologie fang. Séminaire de Dialectologie organisé par le Lascidyl de l'ENS du 1-6 Décembre 1997. Libreville.

Medjo-Mvé, P. 1997c. Interaction ton et quantité vocalique dans le parler fang de la région de Cocobeach. iBoogha 1: 151-165. Libreville : Les Editions du Silence.

Mouguiama-Daouda, P. 1997. La substitution peut-elle expliquer l'unité linguistique du groupe myene? Ibooga 1: 167-202. Libreville: Les Editions du Silence.

Ndinga-Koumba-Binza, H.S. 2000. Phonologie du civili de Mayumba: langue bantu du Gabon (H12a). Libreville : Université Omar Bongo. Mémoire de Maîtrise. 
Nyangone Assam, B. 2001. La lexicographie dans l'apprentissage des langues gabonaises. J.D. Emejulu (éd.), Eléments de Lexicographie Gabonaise. Tome 1: 187-205. New York: Jimacs-Hillman Publishers.

Nzang-Bié, Y. 2001. Vers une éducation multilingue au Gabon: première approche. Revue Gabonaise des Sciences du Langage/Gabonese Journal of the Language Sciences. №2: 1729. Libreville: GRELACO/Université Omar Bongo.

Nzang-Bié, Y. 2002. Le corpus lexicographique dans les langues à tradition orale : le cas du dialecte fang-mekè. Lexikos 12:221-226. Stellenbosch. WAT.

Nzang-Bié, Y. 2008. Pré-enquête pour une standardisation du groupe linguistique fang : la question du choix du dialecte de référence. Eléments de description des langues $d u$ Gabon (édité par D.F. Idiata):191-208. Libreville. Les Editions du CENAREST.

Petyt, K.M. 1980. The study of dialect: An introduction to dialectology. London: Deutsch.

Posner, R. 1994. French. R.E. Asher (Ed.). The Encyclopedia of Language and Linguistics, vol 3: 1299-1301. Pergamon Press.

Poulos, G. \& C.T. Msimang. 1998. A linguistic analysis of Zulu. Cape Town: Via Afrika. Raponda-Walker, A. 1998. Les langues du Gabon. Libreville: Editions Raponda-Walker.

Romaine, S. 1994. Dialect and Dialectology. R.E. Asher (Ed.). The Encyclopedia of Language and Linguistics, vol 2: 900-907. Pergamon Press. 


\section{RECOMMANDATIONS}

Depuis 1983, lors des Etats Généraux de l'Education Nationale et de la Formation, le Gabon s'est engagé à faire de ses langues et des cultures qu'elles véhiculent, la pierre angulaire de son développement. Mais le constat aujourd'hui est que la traduction de cette résolution en actes concrets se fait toujours attendre: les langues gabonaises ne sont encore pas insérées dans le système éducatif, les discussions sur l'adoption d'un alphabet orthographique unifié et utilisé par tous n'ont pas encore abouti, la majorité des langues gabonaises reste à standardiser, le nombre exact des langues nationales reste encore à déterminer, etc. Les conséquences d'une telle situation empêchent le développement effectif des langues gabonaises, c'est-à-dire l'intégration de ces dernières dans le concert linguistique international.

C'est un fait avéré que le développement des pays est intimement lié à celui de leur culture. Pour que le Gabon puisse enfin prendre son envol sur le plan économique et social, il se doit de prendre des dispositions en vue de rattraper le temps perdu. L'heure n'est donc plus au discours, mais à la mise en place de politiques linguistiques concrètes. En tenant compte des propositions existantes, l'adoption et l'utilisation d'un système alphabétique unifié doit aujourd'hui être le cheval de bataille des chercheurs et décideurs gabonais.

En prenant l'initiative d'écrire cet ouvrage, nous voulons à notre manière contribuer au processus de développement des langues gabonaises par des propositions concrètes susceptibles d'une application immédiate dans le contexte actuel.

Par rapport au concept d'alphabet unifié, nous sommes parvenus à la conclusion qu'il faille adopter les graphèmes proposés par le Rapport Final de la Session de Concertation sur l'Orthographe des Langues Gabonaises d'avril 1999. Néanmoins, nous émettons quelques réserves pour ce qui est de la notation du son [ə] par le symbole a (schwa). Pour ce son, Raponda-Walker (dans l'article intitulé "L'alphabet des idiomes gabonais" publié en 1932 et réédité par la Fondation Raponda-Walker en 1998) propose le ë. Nous estimons que ce graphème est approprié pour transcrire le schwa en lieu et place du symbole phonétique.

L'autre réserve se situe au niveau de la notation des tons sur le plan orthographique. Pour ce qui nous concerne et par rapport à ce qui existe ailleurs, (par exemple dans les pays qui ont déjà une large expérience dans l'écriture de leurs langues respectives par un système orthographique approprié : Cameroun, Afrique du Sud, Botswana, Lesotho, Nigeria, Rwanda, Sénégal, République Démocratique du Congo, etc.) nous proposons que la notation des tons soit 
réservée au niveau des transcriptions phonétiques alors que sur le plan orthographique une transcription sans ton est jugée plus pratique pour le grand public. Toutefois, nous relevons le fait que tout système alphabétique est perfectible en fonction des besoins nouveaux surtout lorsqu'il est au stade expérimental. Par exemple, des recherches ultérieures pourraient mettre en évidence la nécessité de tenir compte d'autres réalités des langues gabonaises (en particuliers les faits prosodiques).

Par ailleurs, nous estimons aussi que des initiatives telle que celle de la Fondation Raponda-Walker, à travers l'enseignement à titre expérimental de quelques langues gabonaises au moyen de la méthode Rapidolangue, sont à encourager. Elles ont le mérite d'avoir lancé un projet (le développement des langues nationales) qui nécessite d'être mené jusqu'à son terme. En supervisant cet enseignement le Ministère de l'Education Nationale a implicitement apporté son cachet officiel et scientifique à l'expérimentation Rapidolangue.

Enfin, il faut souligner que quels que soient les efforts des chercheurs et les propositions faites par ces derniers les choses ne peuvent avancer que si des décisions politiques, allant dans le sens de l'application effective de ces propositions, sont prises.

Jacques Hubert

Paul Achille Mavoungou

Thierry Afane Otsaga

Léandre Serge Soami

Hugues Steve Ndinga-Koumba-Binza 


\section{ANNEXES : \\ PROPOSITIONS POUR L'ORTHOGRAPHE}




\title{
Propositions pour l'Orthographe du Yilumbu
}

\author{
Paul Achille MaVoungou
}

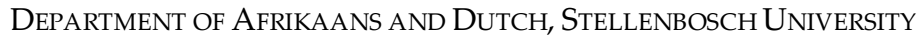

Departement Des Sciences du LANGAge, Universite OMAR Bongo

(moudika2@yahoo.fr)

\section{GRAPHEMES UTILISES ET EXEMPLES}

LES VOYELLES

\begin{tabular}{|c|c|c|c|}
\hline graphèmes & Majuscules & $\begin{array}{c}\text { Exemples en } \\
\text { yilumbu }\end{array}$ & gloses \\
\hline $\mathbf{i}$ & $\mathrm{I}$ & (Ghâng.) fiti & "colère" \\
\hline $\mathbf{i i}$ & $\mathrm{II}$ & "les pieds" $)$ miilu & "espèce de petit poisson \\
d'eau douce"
\end{tabular}

N.B: Le graphème a se réalise tantôt [a] en position préfixale et radicale (ou encore en finale dans les phrases inachevées ou interrompues), tantôt [ə] ailleurs. 


\section{LES CONSONNES}

\begin{tabular}{|c|c|c|c|}
\hline graphèmes & Majuscules & Exemples yilumbu & gloses \\
\hline $\mathrm{p}$ & $\mathrm{P}$ & (Men.) mapapi & "ailes" \\
\hline b & B & (Ghâng.) bwaala & "village" \\
\hline $\mathrm{mb}$ & MB & mbaala & "civette" \\
\hline $\mathbf{t}$ & $\mathrm{T}$ & taata & "père" \\
\hline d & $\mathrm{D}$ & $\begin{array}{ll}\text { (Ghâng. } & \text { Emprunt) } \\
\text { datoola } & \end{array}$ & "docteur" \\
\hline nd & ND & (Men.) ndaghu & "maison" \\
\hline $\mathrm{ndz}$ & NDZ & $\begin{array}{ll}\text { (Ghâng. } & \text { Variante) } \\
\text { ndzubu } & \\
\end{array}$ & "maison" \\
\hline $\mathbf{k}$ & K & kaaka & "grand-parent" \\
\hline g & G & (emprunt) gatu & "gâteau" \\
\hline gh & GH & ughaanga & "saisir, attraper" \\
\hline$\underline{\text { ng }}$ & $\underline{N G}$ & ngaanga & "devin-guérisseur" \\
\hline $\mathrm{f}$ & $\mathrm{F}$ & ufuta & "payer" \\
\hline vh & $\mathrm{VH}$ & vhavha & "ici" \\
\hline $\mathrm{mf}$ & MF & (variante) mfula & "pluie" \\
\hline $\mathbf{m v}$ & MV & mvula & "pluie" \\
\hline s & $S$ & usala & "travailler" \\
\hline $\mathbf{z}$ & $z$ & (Ghâng. emprunt) zeeta & "graisse, huile" \\
\hline $\mathrm{nz}$ & NZ & nzala & "faim" \\
\hline nts & NTS & (variante) ntsala & "faim" \\
\hline ny & NY & (Men.) nyama & "viande; animal" \\
\hline 1 & $\mathrm{~L}$ & ulaamba & "préparer" \\
\hline $\mathbf{r}$ & $\mathrm{R}$ & (Men.) rolu & "sommeil" \\
\hline $\mathbf{w}$ & W & uwaaba & "pêcher" \\
\hline $\mathbf{y}$ & Y & (Ghâng.) yilu & "sommeil" \\
\hline
\end{tabular}




\section{UTILISATION DES MAJUSCULES}

Avant de parler de l'usage de lettres majuscules pour les noms de lieux, clans, personnes, etc. Il convient de rappeler le truisme suivant: Une phrase commence par une lettre majuscule et s'achève par un point: Nzwengiatsifu mo muramba.

Noms de personnes, de clans (tribus), de villages et dénominations des groupes ethnolinguistiques et de leurs langues respectives.

Tous les termes composés représentant des anthroponymes en yilumbu s'écrivent conventionnellement avec un trait d'union. Chaque constituant du mot composé commence par une lettre majuscule. Les noms de clans (tribus), de régions, villes ou pays, de villages, et des groupes ethnolinguistiques ainsi que leurs langues respectives commencent par une lettre majuscule.

\section{EXEMPLES:}

Anthroponymes: Disuvha-di-Koondi; Iiílu-Mubeembi; Mabika-maMbadinga...

Noms de clans: Imondu; Basuumba; Baduumbi...

Noms de régions, villes ou pays: Kulamutu; Masaanga; Fwaala...

Noms de villages: Bilaanga; Muvheemu; Paanga Bikodi...

Noms des groupes ethnolinguistiques: Bapunu; Bapamu; Bawusa...

Dénominations des langues: Yipamu; Yimbaamba; Yingleesa (ou encore Yingesi)...

\section{LES JOURS DE LA SEMAINE}

Les termes désignant les jours de la semaine en yilumbu commencent par une lettre majuscule: Mutoonu, Mudughu, Musiilu; (Men.) Tsona; (Ghâng.) Dumiingu; Sabala...

\section{DIVISION DES MOTS}

Préfixes (nominaux et verbaux), formatifs et extensions

Les préfixes, les formatifs et les extensions sont joints au radical verbal ou au thème nominal pour former un seul mot. Par contre, les mots composant une phrase sont écris séparément.

Exemples: (Men.) Bamaba na misosu; (Ghâng.) Abu tukusilaanu?

\section{LES PREFIXES LOCATIFS AVEC NOMS}

Les préfixes locatifs de classe 16 vha- avec noms s'écrivent en un seul mot: vhoyilu ou encore vhayilu. 
N.B. Les préfixes pronominaux o- (classe17) et mu- (classe 18) utilisés avec des noms ayant une valeur locative s'écrivent de façon disjonctive et non de manière conjonctive.

Exemples: (Ghâng.) o yitanda, (Men.) o dikasa...

\section{LES PRONOMS POSSESSIFS AVEC LES TERMES DE PARENTE}

Les pronoms possessifs utilisés avec des termes de parenté s'écrivent conjointement.

Exemples: mwanaami; nzubwaami; ...

Les démonstratifs

Les démonstratifs constituent une catégorie lexicale à part entière et doivent par conséquent s'écrire de façon disjonctive. Exemples: aghu; ghuna; ghunaa; etc. Cependant, les pronoms démonstratifs utilisés avec des noms s'écrivent conjointement: mutwaghu; mwanaaghu...

\section{LES ADVERBES}

Les adverbes constituent une catégorie lexicale autonome et c'est pourquoi ils s'écrivent de façon disjonctive. Exemples: tiinu; (Men.) biingi; (Ghâng.) pweela, etc. De manière similaire, les adverbes interrogatifs Ana?; Avhe?; Amubi?; Abu? ... s'écrivent conjointement.

\section{APOSTROPHE}

L'apostrophe est utilisée pour indiquer un phénomène d'élision:

Ibonga swe atsikota 'tsi nyosi.

Nongu mu 'wuma urombila dyaambu.

\section{LES TONS}

Deux types de tons sont attestés en yilumbu, à savoir: les tons ponctuels et les tons modulés. Les deux tons ponctuels du yilumbu sont le ton haut (noté par un accent aigu) et le ton bas (noté par un accent grave). Les deux tons modulés du yilumbu sont le ton montant (noté par l'inflexe) et le ton descendant (noté par un accent circonflexe). Les tons sus-mentionnés ne sont pas pris en compte au niveau de l'orthographe. Ils sont réservés aux études scientifiques et universitaires.

\section{EXEMPLE DE TRANSCRIPTION (CONTE)}

Iboonga ya bwaali na ikuumbu 
Iboonga atsiweenda ayenaroomba pira mughetu. Mughetu ghuna akativhita maweela. Nge ke usali isina usoweela mughetu be ghuna. Ti: Pili bi mughetu be ghuna? Ti: Mughetu ta Nza Puungu. Bibulu byootsu batsyeyidama ghuna.

Iboonga atsiweenda. Ti: Mi tsiwiitsa muyitsiweela ikoonda mwaana ta Nza Puungu. $\underline{\text { Oo! }}$ Mughetu vhana rongii ghooghu vho yilu dibange. Ngeeyu ta Nza Puungu uweela mwanaandi mi ifwaana umona maku mwaali ma paasa. Iboonga ti: Ka mbaana buta! Oon! Maruundu ma ta Nza Puungu ali mo ghoghatsa. Iki abu ditwaminaanga? Uya pi dyeela. Iboonga ti: Yo bootsu bayitsaanga batu bakulini. Mughetu ghuna mi nimuweela. Batsimuvhaana buta. Ka mi mweeni! Oo! Mona tsinzayi wali atsina. Musiru a ayi mwaayi. Ndokwaanu! Batsikota musiru. Batsibasigha mo taandu. Aa! Bamona: Aghu! Bali vhaana ka mwa mwa mwa: Tsipaasa. Ya atsikaamba tsinzayi: Talaanu yẹ! Vhole dumona mi vhanalegha tsipaasa yenu dufwaana ughuulu buboti. Dukusyangaanga: Bivholẹ! Bivholẹ! Bivholẹ! Utubilaanga otsi murima. Ee! Baana beeni batsilabule. Iki utuba otsi murima. Iboonga mwa mwa atsimusindila twa. Aghu! Tsinya yo bwaali. Atsisi ti batsyamugha. Aghu! Atsimuyighigha: yo bwaali. Atsiyighigha: batatu. Ti durangunu du taata atsingaamba dukalefwaana. Iboonga atsifila. Atsifila. Atsifila. Atsifila. Maku mwaali na yina itsidughu. Na baana bana mi ivabaghuulu utubilaanga otsi murima. Sungu moosi o dikaata ti: Yaayi inamana. Nge unaweenda na iboonga. Ti bu? Oon! Duraangu taata atsivhaana, atsivhyoosi. Iboonga suruwa ya ka atsimagha muyetaana mughatsi vhoyilu. Ika samba sambaa. Taata Nzaambi ti: Ngeentsa! Nge iboonga utsibagha. Bo mwanaami! Mi vhana ikya na iwuma nyubetuba. Maruundu ma Nza Puungu unaweenda. Batsimuseenda. Yabe tsusu, ibadaangu, tsikoombu, mandomba, tsipoonzi na birevha. Batsiboonga maku mwaali ma batu munata biima bina. Yabe iboonga na mughatsi ombusa nga. Ikuumbu aghuulu mutsaangu ti: Mughatsi be ghuna iboonga anaboonga. Ti: A! Ivhuusi! Biriimbi ghuna abu atsisi? Ikuumbu ti: Mi le muwivhula vho nzila. Batu uvhyoghaanga na mifuna. Mbolwaanu! Ye mbolwaanu! Bamesi ti: O! Ya ikuumbu ya bi avasi vhana? Isalu bi ya avasala vhana? Ka itsighuulu tsaangu ti iboonga anaweela kala mughetu. Ti: Ibeena! Bali o mbusa. Uwiitsa bavawiitsa. Tya: Ayitsa! Ya ti: Iboonga nge dyeela bi utsibagha muye weela mughetu ghuna? Iboonga ti: Mughetu atsintsola. Ik'abu? Ti: Mughetu ghughu sa waaghu vhe. Awaami. Oo! Iboonga ti Anane?

Ti: Yiina! Iboonga ti: Mi sana dyaambu. Vangi mi itsitsogha mu uweela mughetu ghuna. Ya akati yi yootsu ke iliighu ilaambu. Akati yi yootsu ke iliighu veenga nyosi. Oo! Mi byootsu bina o bwaala bwaami. Oo! Atsimupatula mughatsi. Ti: Vhasali dyaambu. Unubo kala mughetu maambu pi. Duvhusu akati tsiinga vho mbaatsu. Ta Nzaambye! Ka mwanaaghu o iboonga atsiweela, ikuumbu aneyepatula iboonga mughetu. Ta Nzaambi ti: Isi bu? Meri ibeena mwanaami opodu ukala na ikuumbu? Batsilelamu vhana. Basamuweela. A! Yobe baluligha ghuna! Iboonga: Abu nyusiila? Iboonga miisu mo teviha nana amona mwa muru ikula ibeli vhana nyosi. Iboonga ti: Minu vho yilu uwe pi. Ika dyeela bi uvhasi vhana? Amona nyumfu vheee. 
Atsimutela. Nyumfu atsituughu. Ti bu? Aa! Mbaatsi ukumbonaanga vhavha ika mukwiilyẹ! Abu dibiila? Minu Ngo anapatula mughatsi. Iboonga anakaamba kala mughatsi vho nzila: Nge usana iliighu isuusu uyi. Veenga nyosi uvhayi. Nyumfu ti: Mfuta! Ti: Nyufuta. Ti: Ndata yesuusa mi vho kasyaati nyosi! Nyumfu ka te atsinaangula iboonga. Ka vhana nyosi. Iboonga swe atsikota'tsi nyosi. Zeeta uyidamaanga na vhotsi. Ngo utela mughatsi. Mughatsi dukuruu. Utuula biliighu ubili pi. Dukuruu. A! Ivhuusi mwanaaghu iboonga! Biriimbi! Kantsi mughetwaghu akati yi iliighu isuusu veenga nyosi. Ngo aka vhana muyenaroomba nyosi. Aa! Amona nana mo tevha tsinyosi. Iboonga atsikaamba nyumfu: Ufwaana uweenda unaa. Umonaanga. Ti: Oọ! Ngo ka atsikaanga dughootsi. Ik' uweenda muyebuka nyosi mughatsi. Ya Ngo asabekipa tsinyosi. Murima ka muleemu nuumba anasyaala. Nuumba akati yi yootsu ke iliighu veenga nyosi. Yitsaanu! Aaa! Mi nafwa! Ikumbu ti: Oo! Mi tsiwiitsa muyitsiroomba ubuka nyosi mughatsi bi binantsiimba? Iboonga ti: Aghu ami. Aa! Tevha ik'ubweela ukula. Ti: Nyuunga ufwaana monaanga! Nyuunga ka de. Tevha ukulaanga. Ikuumbu ti: Inafwa! Yitsaanu! Ya aka vho yilu. Ana obotuugha vhotsi muyemulaanda ghuna? Myooghu mi myooli mike nduruwee. Atsibuunga. Atsibuunga. Atsibuunga. Aghu! Iboonga ti: Anatogha. Atsimupatula. Akawiitsa monaanga dighayi ube yaaba ti mutu lwaa vhotsi. Bibentsigha muru, myooghu uyenatatabula. Iboonga ti: Nyuunga! Ya ti: Oo! Atsibasigha. Atsiwiitsa wu wu wu tena vhana muru nyosi. Atsiboonga iboonga. Atsibemuwubatana wu wu wu ngu na vhotsi. Iboonga ti: Aa! Dibotyẹ! Yanti: Diboti di maamba usavhule tsipuunda utsiwe. Nge untsivaanga pira diboti. Nyuunga ti: Mi tsighuulu wa nana utsibuka noongu. Kantsi mfuta! Iboonga ti: Nge utsiyaabẹ? Ya: Lyongu! Ya: Dede buloongu bumana nge usoburulu vhotsi. Ukuburulaanga vhoyilu. Vangi mwiri yina ivaghuvhaana: Mufumwẹ! Avhana uburulaanga. Nyuunga: Tibu? Nge usamonẹ? Kusu bavayemutsakulaanga. Inooghu bavamutsakulaanga. Vangi mwiri be ghuna itsighuvhaana baana baaghu bobangughaanga usomona ti mutu owiitsa muboonga baana baaghu ti muyelaamba. Nyuunga ya: Ibeena! Mi tsisiinga. Ti: Bweela! Oo! Vhumu swiii! Ti: Nge utsiyaabe? Ti: Lyongu! Ti: Diloongu dyo, maba mo amaaghu ngeeyu. Diba dinasagha uyangaanga tsingatsi. Mughatsyaaghu iloongu ineni bafwaana unataanga dina dyaaghu ti: Beghaanu mughaanda ubola nyuunga. Dimweegha dinẹ! Vhole uyenaghangalaanga, nataanga mikaandi tsina diloongu dyo tsimbari dikenalavhaanga. Bakenabeghaanga dina dyaaghu o meesu. Nyuunga ti: Ibeena! A dibaandu uvamona nyuunga ti aka vhoyilu mwiri uvamughuulu: Han. Han. Han. Ti: Ngeentsa Iboonga! Nge unumbaana kala pira busina. Iboonga anabuka nyosi. Anyetaana mughatsyaandi. Oo! Nuumba aka vhana roongi! Ika samba samba sambaa! Batsiweenda o bwaala. Noongu mu ‘wuma urombila dyaambu.

Conte collecté en 1993 auprès de MOUSSAVOU MOUSSOUAMI plus connu sous le pseudonyme de SAMANGWANA. 


\title{
Propositions pour l'Orthographe du Fang
}

\author{
THIERRY AFANE OTSAGA \\ DEPARTEMENT DES SCIENCES DU LANGAGE \\ UNIVERSITE OMAR BONGO, LIBREVILLE \\ (afanotsaga@gmail.com)
}

\section{GRAPHEMES UTILISES ET EXEMPLES}

Les graphèmes orthographiques que je propose pour la transcription du Fang (ntumu) sont ceux de la Nouvelle Orthographe des Langues Gabonaises qui est le résultat de la Session de Concertation des Experts tenue à Libreville en avril 1999. Le seul graphème de cette orthographe qui n'est pas retenu ici est le ə, car il est identique au symbole phonétique. Pour transcrire ce symbole orthographiquement je suggère de le remplacer par le graphème ë proposé par le premier linguiste gabonaise RapondaWalker et repris dans la méthode Rapidolangue (Editions Raponda-Walker, 1999).

\section{LES VOYELLES}

\begin{tabular}{|c|c|c|c|c|}
\hline \multirow{2}{*}{$\begin{array}{l}\text { Voyelles } \\
\text { de l'alphabet } \\
\text { orthographique }\end{array}$} & \multirow{2}{*}{$\begin{array}{l}\text { Voyelles } \\
\text { de l'alphabet } \\
\text { phonétique } \\
\text { (API) }\end{array}$} & \multicolumn{3}{|l|}{ Exemples } \\
\hline & & $\begin{array}{l}\text { Ecriture } \\
\text { orthographique }\end{array}$ & $\begin{array}{l}\text { Transcription } \\
\text { phonétique }\end{array}$ & $\begin{array}{l}\text { Equivalents en } \\
\text { français }\end{array}$ \\
\hline i & [i] & amin & [ámìn] & "avaler" \\
\hline$\underline{\mathrm{u}}$ & {[]} & ekuri & [ékùrì] & "le piège" \\
\hline$\underline{\mathrm{e}}$ & {$[\varepsilon]$} & bile & [bìlé:] & "les rêves" \\
\hline e & [e] & eve & [èvé:] & "la soif" \\
\hline$\ddot{\mathrm{e}}$ & [ə] & avëp & [àvòp] & "le froid" \\
\hline a & [a] & mvam & [mvám] & "le grand-parent" \\
\hline$\underline{\mathrm{o}}$ & [0] & mbom & [mìbóm] & "la belle-fille" \\
\hline o & [o] & dzom & [dzóm] & "la chose" \\
\hline $\mathrm{u}$ & {$[\mathrm{u}]$} & abum & [àbùm] & "le ventre" \\
\hline
\end{tabular}

N.B. Il est important de noter que les voyelles peuvent être longues ou nasalisées en fonction de leur contexte d'apparition à l'intérieur d'un item lexical. J'estime que ces 
voyelles longues ou nasalisées ne font pas parties des symboles alphabétiques, mais ils peuvent être notés phonétiquement lorsqu'ils ne sont pas pertinents sur le plan phonologique et orthographiquement lorsqu'ils sont phonologiquement pertinents.

\section{LES CONSONNES}

\begin{tabular}{|c|c|c|c|c|}
\hline \multirow{2}{*}{$\begin{array}{l}\text { Consonnes } \\
\text { de l'alphabet } \\
\text { orthographique }\end{array}$} & \multirow{2}{*}{$\begin{array}{l}\text { Consonnes } \\
\text { de l'alphabet } \\
\text { phonétique } \\
\text { (API) }\end{array}$} & \multicolumn{3}{|l|}{ Exemples } \\
\hline & & $\begin{array}{l}\text { Ecriture } \\
\text { orthographique }\end{array}$ & $\begin{array}{l}\text { Transcription } \\
\text { phonétique }\end{array}$ & Equivalents en français \\
\hline$b$ & $b$ & obon & [òbón] & "le menton" \\
\hline $\mathrm{d}$ & $\mathrm{d}$ & adan & [ádàn] & "monter" \\
\hline $\mathrm{f}$ & $\mathrm{f}$ & fam & [fám] & "homme" \\
\hline g & g & ngan & [ngan] & "le caïman" \\
\hline $\mathrm{k}$ & $\mathrm{k}$ & $\underline{\mathrm{nku}}$ & {$[\mathrm{\eta ku}]$} & "Le sel" \\
\hline j & $d_{3}$ & ajenny & [ádzعn] & "la pauvreté" \\
\hline 1 & 1 & alu & [àlù] & "la nuit" \\
\hline $\mathrm{m}$ & $\mathrm{m}$ & aman & [àmãj] & "a joue" \\
\hline $\mathrm{n}$ & $\mathrm{n}$ & anamë & [ánámə] & "monter" \\
\hline$\underline{\mathrm{n}}$ & 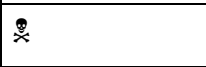 & ntsin & [ntsín] & "le tisserand" \\
\hline $\mathrm{p}$ & $\mathrm{p}$ & ekop & [èkòp] & "la peau" \\
\hline $\mathrm{r}$ & $\mathrm{r}$ & okiri & [òkírí] & "demain"” \\
\hline $\mathrm{s}$ & $\mathrm{s}$ & asum & [ásùm] & "commencer" \\
\hline$t$ & $\mathrm{t}$ & etam & [ètám] & "la solitude" \\
\hline $\mathrm{v}$ & $\mathrm{v}$ & mvën & [mvən] & "la pluie" \\
\hline$w$ & $\mathrm{w}$ & awom & [àwóm] & "dix" \\
\hline $\mathrm{y}$ & $y$ & eye & [èyع] & "la lèvre" \\
\hline $\mathrm{z}$ & z & azu & [ázù] & "venir" \\
\hline gh & $\gamma$ & abëghë & [àbəyə] & "porter" \\
\hline $\mathrm{sh}$ & $\int$ & ashëmë & [àfəmə] & "chasser" \\
\hline ny & $\mathrm{n}$ & abiny & [àbìn] & "attraper" \\
\hline
\end{tabular}


N.B. En fang il est aussi possible de trouver des unités consonantiques complexes telles que dz (dzeny "l'ongle"), gb (ngbo:), kp (okpon), ts (tsí:)

\section{UTILISATION DES MAJUSCULES}

Comme dans toute écriture formelle, il convient de rappeler l'évidence selon laquelle une phrase commence par une lettre majuscule et s'achève par un point: Oshin wa lot nkyeny.

\section{NOMS DE PERSONNES, DE CLANS (TRIBUS), DE VILLAGES ET DENOMINATIONS DES GROUPES ETHNOLINGUISTIQUES ET DE LEURS LANGUES RESPECTIVES.}

Tous les termes composés représentant des anthroponymes en fang s'écrivent conventionnellement avec un trait d'union. Chaque constituant du mot composé commence par une lettre majuscule. Les noms de clans (tribus), de régions, villes ou pays, de villages, et des groupes ethnolinguistiques ainsi que leurs langues respectives commencent par une lettre majuscule.

\section{EXEMPLES:}

Anthroponymes: Afan-Otsagh; Angwe-Ntutum; Obam-Nna...

Noms de clans: Yëmëkak; Esan; Edzoma...

Noms de régions, de villes ou de pays:; Minvul; Oyëm; Fala.

Noms de villages: Mëbëm; Esoñ ; Eboman...

Noms des groupes ethnolinguistiques: Bëfan; Bëpunu; Bëdzabë...

Dénominations des langues: Fang; Punu...

\section{LES JOURS DE LA SEMAINE}

Les termes désignant les jours de la semaine en Fang commencent par une lettre majuscule: Sonë.

\section{APOSTROPHE}

L'apostrophe est utilisée pour indiquer un phénomène de pose dans la prononciation: $N^{\prime} n a, N^{\prime} n a n g . .$.

\section{LES TONS}

Afin de ne pas charger les textes et les rendre répulsifs pour les non-initiés des questions linguistiques, la transcription tonale est évitée en fang sur le plan orthographique. Elle est réservée aux études scientifiques et universitaires surtout au plan phonétique. 


\section{EXEMPLE DE TRANSCRIPTION ORTHOGRAPHIQUE DE L'HISTOIRE SUR L'ORIGINE DU NOM DU VILLAGE AKOAKAM (MICHEL VOLTZ, 1990: 171).}

Engan to Akwakam ndęmbëki eyola na akwakam. Akwakam embë eyola na Mëndun. Mëndun mënë bile. Mbor afan embë vomë të. Andun enë mor ele, dafe afan yë bëbe oshiny.Andung dafe evomë si enë ntaghan. Edë Ekogha Ndon yë ebwan beny bëbeny bënga alon dze ve Akwakam ënë eyola minëna. Mbu 1904 Kapiten Weber anga alon Oyem. Anë mintan minga amanëkom anë bënë bëvë bidzi na: dze sësë dayien so bikwan, ëmbon, ndogho, nkok, nkkoro kwas, nkkoro dzit...Anë Mëndung anga tëlë ëngura minënga na ewo wake wanyon bidzi adze ete ekëkë mintan. Minëna të ambë eyola na 'Akwakam-Akoma, ngwan Odzip, yë a ngwëma. Eenyë anga ajwe nsama binëna bënga vëvë mintan yë bëkwan akum bidzi. Minëna të ambë ngënge. Eyon sësë bënga akë vë bidzi, Mëndung enyë anga adan bëlë abwiny yë fë mbana bidzi, Ambë ewogha abwiny ebë bëjwe. Benga alue fë edze de eyola nzeny, benga alue dë eyola minëna të na akwakam. Akoro yë eyon të edze enga anyon eyola Akwakam. 


\title{
Propositions pour l'Orthographe du Civili
}

\author{
Hugues STEVE NDINGA-KOUMBA-BINZA \\ CENTRE FOR TEXT TECHNOLOGy (CTEXT) \\ NORTH-West UNIVERSITY, POTCHEFSTROOM CAMPUS \\ (22602560@nwu.ac.za)
}

Pour l'orthographe du civili, je propose un système qui s'organise autour d'un alphabet simple de 24 symboles, à savoir 6 voyelles et 16 consonnes. Le critère principal de l'établissement ce système d'orthographe est l'accessibilité auprès des potentiels utilisateurs qui peuvent locuteurs natifs ou apprenants. Les particularités phonético-phonologiques de la langue sont reprises dans le cadre des règles d'écriture et de lecture.

\section{LES VOYELLES}

LES SYMBOLES ORTHOGRAPHIQUES DES VOYELLES

\begin{tabular}{|c|l|l|l|l|}
\hline & Minuscule & Majuscule & Exemples & Gloses \\
\hline $\mathbf{1 .}$ & $\mathbf{a}$ & $\mathbf{A}$ & liba & «le palmier à huile » \\
\hline 2. & $\mathbf{e}$ & $\mathbf{E}$ & ndebu & «filet de pêche » \\
\hline 3. & $\ddot{\mathbf{e}}$ & $\ddot{\text { E }}$ & bëbakëlë & «les garçons » \\
\hline 4. & $\mathbf{i}$ & $\mathbf{I}$ & livapi & « aile » \\
\hline 5. & $\mathbf{0}$ & $\mathbf{O}$ & tolu & «sommeil » \\
\hline $\mathbf{6 .}$ & $\mathbf{u}$ & $\mathbf{U}$ & munu & «bouche » \\
\hline
\end{tabular}

\section{LES REGLES D'ECRITURE DES VOYELLES}

\section{LA LONGUEUR VOCALIQUE}

Toutes les voyelles ont des correspondantes longues, à l'exception du schwa (ayant le symbole orthographique ë). La longueur vocalique, phonologique dans la langue, se marque par le redoublement de la voyelle seulement pour les paires minimales.

Exemples:

$\begin{array}{lll}\text { N'teela «le chasseur» } & \text { versus } & \mathrm{N}^{\prime} \text { tela «la taille» } \\ \text { Mbaasi «l'ami» } & \text { versus } & \mathrm{Mbasi} \text { «demain» } \\ \text { Loosu «le riz» } & \text { versus } & \mathrm{Losu} \text { «la saleté du poisson» }\end{array}$


Mais, la voyelle n'est pas redoublée lorsqu'elle précède un complexe consonantique nasal ou quand elle vient après une semi-consonne (w ou y). Ce sont des contextes où la longueur est prévisible dans le système phonologique de la langue. Les lecteurs non natifs doivent être instruits de ce fait par une règle de lecture.

Exemples :

$\begin{array}{ll}\text { Mweni } & \text { "l'étranger» } \\ \text { Lwibë } & \text { "l'oubli» } \\ \text { Lyesu } & \text { "l'oil » } \\ \text { Ngandu } & \text { "crocodile» } \\ \text { Ndimbu } & \text { "caoutchouc» } \\ \text { Ndungu } & \text { "tambour» }\end{array}$

\section{LA VARIATION A ET Ë.}

En position préfixale, les graphèmes a et ë varient librement. Mais on a besoin de tous les deux dans l'écriture du civili. Le premier apparaît systématiquement dans la voyelle accentuée du radical, et le deuxième qui est le plus fréquent dans la langue occupe toutes les autres positions (non accentuée).

\section{LES VOYELLES NASALES}

La voyelle est généralement nasalisée devant les consonnes mi-nasales. Ce contexte suffit à lui seul pour représenter de manière orthographique la nasalisation qui n'est pas phonémique en civili.

Exemples :

$\begin{array}{ll}\text { Ncenzu } & \text { "la plaie, la maladie» } \\ \text { Ntangu } & \text { "le soleil, la période » } \\ \text { Sindonga } & \text { "les assiettes } \\ \text { Milunga } & \text { «les bracelets }\end{array}$

\section{LES REGLES DE LECTURE DES VOYELLES}

Toutes les voyelles se prononcent comme dans la plupart des langues bantu avec les précisions suivantes.

Le graphème ë se réalise [ə] dans toutes les positions où il se présente.

Le graphème e se prononce à la fois [e] et $[\varepsilon]$. La dernière forme est la plus fréquente et apparaît général dans une syllabe fermée.

Le graphème o se prononce à la fois [o] et [0]. Ici également, la dernière forme est la plus fréquente et apparaît général dans une syllabe fermée 
Le graphème $\mathbf{a}$ est prononcé [a] dans les positions où il apparaît.

Les graphèmes $\mathbf{u}$ et $\mathbf{i}$ se prononcent avec un dévoisement lorsqu'ils apparaissent en finale de mot en isolation ou en fin de phrase.

Toute voyelle est généralement prononcée avec une certaine longueur vocalique lorsqu'elle précède un complexe consonantique nasal ou quand elle vient après une semi-consonne (w ou y).

\section{LES CONSONNES}

LES SYMBOLES ORTHOGRAPHIQUES DES CONSONNES

\begin{tabular}{|c|c|c|c|c|}
\hline & Minuscule & Majuscule & Exemple en Civili & Français \\
\hline 1. & b & B & Bëbakëlë & Les garçons \\
\hline 2. & c & C & Micyodu & Les épées \\
\hline 3. & d & D & Dukudakë & Le requin \\
\hline 4. & $\mathrm{f}$ & F & Fofulu & Allumettes \\
\hline 5. & k & K & Kooku & Le bras \\
\hline 6. & 1 & L & Milelë & Les pagnes \\
\hline 7. & $\mathbf{m}$ & $\mathbf{M}$ & Limemë & La chèvre \\
\hline 8. & $\mathbf{n}$ & $\mathbf{N}$ & Cinunu & Le vieillard \\
\hline 9. & p & $\mathbf{P}$ & Mapaapë & Les babouches \\
\hline 10. & $\mathbf{r}$ & $\mathbf{R}$ & Kwartu & La chambre \\
\hline 11. & $s$ & $S$ & Susu & La poule \\
\hline 12. & $t$ & $\mathrm{~T}$ & Kutetikë & Superposer \\
\hline 13. & $\mathbf{v}$ & V & Bëvikë & Les esclaves \\
\hline 14. & $w$ & W & Awu & Celui-ci \\
\hline 15. & $y$ & $Y$ & Fayetë & Tailleur \\
\hline 16. & $\mathrm{z}$ & $\mathrm{Z}$ & Mëzinë & Les noms \\
\hline
\end{tabular}

\section{LES REGLES D'ECRITURE DES CONSONNES}

La nasale palatale [n] se note orthographiquement par l'association des consonnes $n$ et $y$. Exemples : Nyosë (le miel), nyamu (la tortue de mer), kunyefë (être belle).

Toutes les consonnes palatalisées sont également orthographiées dans un complexe consonantique avec $\boldsymbol{y}$. Exemples : Lyesu (œil), myoku (les bras), byalë (les carpes). 
Les consonnes labialisées s'orthographient dans un complexe avec $w$. Exemples: Lwabi (la courroie), kwendë (s'en aller), mwalë (la marée), kubwa (faire une chute).

La nasale syllabique, très fréquente en civili, se marque par la lettre $n$ apostrophée : $\mathrm{n}^{\prime}$ ( $\mathrm{N}^{\prime}$ en majuscule). Elle se distingue ainsi de la nasale qui est à l'initiale d'une minasale. Exemples: N'cyodu (l'épée, pluriel : micyodu), n'cyetu (la femme, pl : bëcyetu), n'kwati (machette, $\mathrm{pl}$ : minkwati), n'teelë (chasseur, $\mathrm{pl}$ : bëteelë).

Les consonnes mi-nasales sont orthographiées, mais ne sont pas listées comme lettres de l'alphabet vili. L'homorganicité permet au locuteur ou à l'apprenant d'identifier les composantes de chaque mi-nasale. On note :

$\begin{array}{lll}\mathrm{Mb} & \text { Cimbolu } & \text { "le crocodile" } \\ \mathrm{Mp} & \text { Mpemu } & \text { "les vents" } \\ \mathrm{Nc} & \text { Sincimë } & \text { "les singes" } \\ \mathrm{Nd} & \text { Ngandu } & \text { "le caïman" } \\ \mathrm{Ng} & \text { Ngangë } & \text { "le guérisseur" } \\ \mathrm{Nj} & \text { Nzunji } & \text { "la bile" } \\ \mathrm{Nt} & \mathrm{Ntangu} & \text { "le soleil" } \\ \mathrm{Nz} & \text { Nzalë } & \text { "la faim" }\end{array}$

\section{LES REGLES DE LECTURE DES CONSONNES}

La consonne c se prononce [t $\left.\int\right]$ comme dans le mot français 'Tchèque'. Exemples: civili (la langue vili), cikwangë (le baton de manioc). Elle a la même réalisation dans la mi-nasale nc comme dans le mot sincimë (les singes)

La consonne nasale ng se prononce [y] comme dans le mot très connu 'nganga'.

Les consonnes sourdes $\mathbf{k}$, $\mathbf{p}$ et $\mathbf{t}$ sont généralement aspirées lorsqu'elles sont précédées d'une nasale comme dans les mots suivants.

$\begin{array}{lll}\text { Simpuku } & \text { [símphúkù] } & \text { les rats } \\ \text { Muntu } & {\left[\text { mú: } \text { thù }^{\text {hú }}\right.} & l^{\prime} \text { Homme } \\ \text { Minkwati } & {\left[\text { mínk }^{\mathrm{h}} \text { wátì] }\right.} & \text { les machettes }\end{array}$

La dernière partie de la mi-nasale $n j$ se prononce [dz] comme le mot anglais ' job'. Elle ne retrouve pas en dehors du complexe nasal. Exemples: Nje (toi), mbunji (la moisissure).

Selon les variantes dialectales et/ou régionales les consonnes $s$ et $z$ peuvent se prononcés respectivement [s] ou [ $]$ ] et [z] ou [3] en variation libre. Exemples : Mbisi 'viande' (Mayumba/Ndindi: [mbísi] ; Loango/Pointe-Noire: [mbíî]), lizinë 'nom' (Mayumba/Ndindi : [lízínò] ; Loango/Pointe-Noire : [lízíṅ̀]). 
La consonne $v$ se prononce tantôt $[\beta]$ tantôt $[v]$ en variation complémentaire. La dernière est celle qui se prononce lorsque la consonne est précédée par une nasale. Mais elle tend à supplanter la première forme, qui devient de plus en rare, à cause de l'influence du français.

\section{LES SIGNES DE PONCTUATION}

\section{LES MAJUSCULES}

Pour tous les noms propres (lieux, clans et tribus, groupes ethnolinguistiques, langues, rivières et fleuves, montagnes, forêts, etc.) et les noms de personnes, la majuscule est portée sur le préfixe nominal. Les jours de la semaine et les noms des mois s'écrivent aussi avec une majuscule.

Exemples :

$\begin{array}{ll}\text { Sabëlë } & \text { «samedi } \\ \text { Lumingu } & \text { «dimanche, la semaine } \\ \text { Mayumbë } & \text { "Mayumba» } \\ \text { Baanyi } & \text { «la lagune Banio» } \\ \text { Badumbi } & \text { "clan Baduumbi» } \\ \text { Bayenji } & \text { "clan Bayeenji } »\end{array}$

Le trait d'union. Tous les termes composés s'écrivent de manière conventionnelle avec un trait d'union.

\section{Exemples :}

Biva-bi-Lwangu « clan Biva-bi-Lwaangu»

Basë-u-Kongu «clan des Bakongo »

Mwa-ci-Kambisi "génie des eaux anciennement vénéré chez les Bavili »

Les points: le point final ou d'abréviation (.) - le point-virgule (;) - les deux points (:) le point d'interrogation (?) - le point d'exclamation (!) - les trois points de suspension (...), ils sont marqués comme en français et remplissent également les mêmes fonctions.

Maamë afumë ku n'situ. «Maman est rentrée de brousse. »

Maamë afumë ku n'situ? «Maman est-elle rentrée de brousse ?»

Maamë afumë ku n'situ, anyikë mëyakë : bitu byalë volë. «Maman est rentré de brousse, puis elle a pilé le manioc : elle est épuisée. »

Mphonzë yi maamë yiwalë yi biimu : mphindë, bitebë, ... byosu biimu bi mboti. « La hotte de maman est pleine de choses : des arachides, des bananes... Toutes des bonnes choses ». 
Les autres signes: la virgule (,) - les guillemets («») - les parenthèses ( ) l'espacement, on les écrit de la même manière avec les mêmes portées sémantiques qu'en français.

Exemples:

N'tinu atubilë abu (yi mwangu) : «Mu fwanë kutungë bwalë bwinu! ». Le chef a dit ceci (avec colère) : "il faut développer votre village! »

\section{L'APOSTROPHE}

L'apostrophe est utilisée pour indiquer un phénomène d'élision, mais aussi la nasale syllabique à l'initiale de certains mots.

Exemples:

Mwan'awu atiinë cikoolë.

Taat'akwe ku londu.

N'cyetu

N'senfu

N'situ
«Cet enfant a fui de l'école ».

«Papa est allé au débarcadère ».

« la femme » (Pluriel : Bëcyetu)

«la banane douce » (Pl : Misenfu)

«la brousse » (Pl : Misitu)

\section{LA DIVISION DES MOTS}

\section{LE MOT}

Le mot en civili est constitué d'un préfixe (nominal ou verbal) et d'un radical (thème nominal ou racine verbale). Les extensions (affixes, suffixes et finale verbale aussi) au radical font également partie du mot.

\section{Exemples:}

$\begin{array}{ll}\text { Kulambë } & \text { "préparer» } \\ \text { Kulambisë } & \text { "faire préparer» } \\ \text { Kulambilë } & \text { "préparer pour quelqu'un» } \\ \text { Kulalambë } & \text { "préparer précipitamment» }\end{array}$

\section{Préfixes (nominaux, verbaux et pronominaux), formatifs et extensions}

Les préfixes (à l'exception des locatifs) et les extensions sont joints au radical du verbe ou du thème nominal pour former un seul mot. Voir les exemples ci-dessus avec Kulaambë.

Les mots dans une phrase sont écris séparément. Exemples : 
Taatë akwe ku bisalu «Papa est allé au travail ».

Ya tinë singasi

«j'ai coupé un régime de palme »

Les pronoms participants, les formatifs et les connectifs sont écrits séparément: ils constituent des mots à part entière.

Exemples :

Libakëlë li nzu «l'homme de la maison»

A kwe yi taatë «il est partiavec papa»

Ya fi bwa "j'ai failli tomber »

\section{LES PREFIXES LOCATIFS AVEC NOMS}

Les préfixes locatifs de classe 16 (fë ou $f a), 17(k u)$ et $18(m u)$ s'écrivent de façon disjonctive. Ceci les distingue des préfixes $m u$ - et $k u$ - des classes respectives 1 et 3 , et 15.

Exemples:

Mu bwatu «dans la pirogue».

Fë n'toku «sur terre»

Ku bwalë «au village, à la maison»

\section{LES PRONOMS PARTICIPANTS ET PERSONNELS}

Les pronoms participants et personnels s'écrivent disjonctivement.

Ya (ou Y dans certaines conditions) pour dire je comme dans ya bwilë mbisi wali (j'ai attrapé deux poissons).

Mi (ou miinu en fin de phrase) pour dire moi ou me comme dans mi be ba monë (je les ai vus)

$\mathrm{Wa}$ (ou $W$ dans certaines conditions) $=\mathbf{t u}$;

Nje (ou njeyu en fin de phrase) = toi

$\mathrm{A}=\mathrm{il}$, elle, lui

Beesu (ou Be en contexte de discours) = nous

Beenu (ou $\mathrm{Be}$ en contexte de discours) = vous

Bawu (ou $B a$ dans certaines conditions) = ils, elles, eux

Exemple:

Baawu banonga sifooyi, balaka miinu «Ils ramassent des boîtes, et les jêtent sur moi». 


\section{LES ADJECTIFS POSSESSIFS}

Les adjectifs possessifs s'écrivent conjonctivement. Mais ils sont le plus souvent apostrophés compte tenu de l'élision vocalique qui les précède et qui est due à leur initiale vocalique.

Exemples:

Mwan'aami «mon fils »
Maam'itu «notre mère »

\section{LES DEMONSTRATIFS}

Les démonstratifs constituent une catégorie lexicale à part entière et doivent par conséquent s'écrire de façon disjonctive. Exemples: awu (celui-ci), wuna (celui là-bas), etc. Ils peuvent aussi être mis en apostrophes dans les contextes d'élision vocalique. Exemples:

$\begin{array}{ll}\text { Libë lina } & \text { "ce palmier là-bas» } \\ \text { Lib' oli } & \text { "ce palmer-ci» } \\ \text { Ngasi osi } & \text { "cette noix de palme-ci » } \\ \text { Singasi sina } & \text { «ces noix de palme là-bas }\end{array}$

L'on remarque l'adjectif démonstratif équivaut à la voyelle a et/ou le morphème na à laquelle s'associe le préfixe nominal du mot désigné. C'est la raison pour laquelle le démonstratif doit être disjoint du mot puisque la forme préfixale est en constant changement.

\section{LES ADVERBES}

Les adverbes constituent une catégorie lexicale autonome et c'est pourquoi ils s'écrivent de façon disjonctive.

Exemples:

$\begin{array}{ll}\text { Tiinu } & \text { «vite» } \\ \text { Mpampë } & \text { «eaucoup } \\ \text { Nyakëlë } & \text { "plusieurs }\end{array}$

\section{LES PRONOMS INTERROGATIFS}

Les pronoms interrogatifs et relatifs s'écrivent disjonctivement. 
$\mathrm{Na}$ ? (pluriel Bana?)

$\mathrm{Ku}$ ?

$\mathrm{Mbi}$ ?

Abu? qui ?

où ?

quoi ? (ou pourquoi?)

Comment? (C'est comment?)

\section{LES TONS}

Sur le plan tonétique, on peut observer en civili quatre tons : le ton bas, le ton haut, le ton descendant et le ton montant. Le ton montant est très rare dans la langue. Le ton haut et le ton bas sont des tons ponctuels très fréquents. Les tons modulés (montant et descendant) se réalisent à la suite d'un phénomène d'allongement vocalique, ils ne sont pas tonologiques.

Les tons du civili ne sont pas transcrits sur le plan orthographique. Ils sont réservés aux études scientifiques.

\section{EXEMPLE DE TRANSCRIPTION ORTHOGRAPHIQUE}

\section{DEVISE DE L'ANCIEN ROYAUME DE LOANGO:}

Likandë li kooku li simbë simbotë sambwali

La paume de la main qui tient sept étoiles

\section{EXTRAIT DE CONTE:}

Fëba n'cyetu ubanga yi solë n'nuni aandi unsolilë. Bunë amanë kuvatë, aba yekë kwenda mu kusakulë. Cilumbë ci mesë akwendë, utalë ku yulu, umonë abu singasi. Naandi ti: osi? »

«Eeh, aku yakë n’bacilë kwandi cisilikilë ci libakëlë wu ku n'tininë singasi

Bo keke yamba bobu, bemonë abu cisilikilë ci libakëlë cyakituukë. N'cyetu benë be n'kwambililë :

«Eeh, ya cisilikilë ci libakëlë, n'tininë kwa singasi osi e! » (minaanu matë) 


\title{
Propositions pour l'Orthographe du Yipunu
}

\author{
LEANDRE SERGE SOAMI \\ INSTITUT DE RECHERCHE EN SCIENCES HUMAINES (IRSH) \\ Centre National de Recherche Scientifique et Technologique (CENAREST), Libreville \\ (sergesoami@gmail.com)
}

\section{GRAPHEMES UTILISES ET EXEMPLES}

\section{LES VOYELLES}

\begin{tabular}{|c|c|c|c|c|c|}
\hline 1. & i & I & [i] & ditoghu & Natte \\
\hline 2. & ii & Ii & [i:] & diimbu & Village \\
\hline 3. & e & E & [e] & temu & Epoque \\
\hline 4. & ee & Ee & [e:] & nzwengi & Colibri \\
\hline 5. & $\underline{\mathbf{e}}$ & E & {$[\varepsilon]$} & yesa & Chance \\
\hline 6. & ee & Ee & {$[\varepsilon:]$} & Yisyeela & Récolteur \\
\hline 7. & a & A & {$[\mathrm{a}],[ə]$} & taji & Père \\
\hline 8. & aa & Aa & [a:] & ejaabi & Il connaît \\
\hline 9. & $\underline{\mathbf{o}}$ & $\mathrm{O}$ & [0] & Uboka & Tuer \\
\hline 10. & $\underline{\text { oo }}$ & Oo & [0:] & Uboola & Ramasser \\
\hline 11. & $\mathbf{o}$ & 0 & [o] & kodu & Nuque \\
\hline 12. & oo & Oo & [o:] & diloongi & Conseil \\
\hline 13. & $\mathbf{u}$ & $\mathbf{U}$ & {$[\mathrm{u}]$} & mutu & Homme \\
\hline 14. & uu & $\mathbf{u}$ & [u:] & nuungi & Plantation \\
\hline
\end{tabular}

Ce tableau présente les symboles orthographiques des voyelles du yipunu. Il contient 14 voyelles dont 10 fermes et 4 ouvertes. Cet inventaire alphabétique se base sur le principe qui veut que chaque son de la langue soit associé à un symbole. L'alphabet du yipunu tient compte de tous les sons vocaliques de la langue avec l'objectif de mettre a la disposition des usagers une orthographe qui tiennent compte de toutes les situations d'écriture.

La voyelle a se réalise [ə] en position finale de mot (en isolation) et de phrase. Elle se réalisera[a] partout ailleurs. 


\section{LES CONSONNES}

\begin{tabular}{|l|l|l|l|l|l|}
\hline $\mathbf{1 .}$ & $\mathbf{p}$ & $\mathbf{P}$ & {$[\mathrm{p}]$} & Putu & Maïs \\
\hline $\mathbf{2}$. & $\mathbf{b}$ & $\mathbf{B}$ & {$[\mathrm{b}]$} & Batu & Hommes \\
\hline $\mathbf{3 .}$ & $\mathbf{m b}$ & $\mathbf{M b}$ & {$[\mathrm{mb}]$} & Mbata & Giffle \\
\hline $\mathbf{4 .}$ & $\mathbf{t}$ & $\mathbf{T}$ & {$[\mathrm{t}]$} & Taba & Mouton \\
\hline $\mathbf{5 .}$ & $\mathbf{d}$ & $\mathbf{D}$ & {$[\mathrm{d}]$} & Diisu & Eil \\
\hline $\mathbf{6 .}$ & $\mathbf{n d}$ & $\mathbf{N d}$ & {$[\mathrm{nd}]$} & Ndasi & Montre-moi \\
\hline $\mathbf{7 .}$ & $\mathbf{k}$ & $\mathbf{K}$ & {$[\mathrm{k}]$} & Kaki & Tonnère \\
\hline $\mathbf{8 .}$ & $\mathbf{g}$ & $\mathbf{G}$ & {$[\mathrm{g}]$} & Galu & Galon \\
\hline $\mathbf{9 .}$ & $\mathbf{g h}$ & $\mathbf{G h}$ & {$[\mathrm{y}]$} & Ghaba & Partage \\
\hline $\mathbf{1 0 .}$ & $\mathbf{n g}$ & $\mathbf{N g}$ & {$[\mathrm{p}]$} & Ngandu & Caïman \\
\hline $\mathbf{1 1 .}$ & $\mathbf{f}$ & $\mathbf{F}$ & {$[\mathrm{f}]$} & Ifumba & Famille \\
\hline $\mathbf{1 2 .}$ & $\mathbf{v h}$ & $\mathbf{V h}$ & {$[\beta]$} & Vhatsi & Par terre \\
\hline $\mathbf{1 3 .}$ & $\mathbf{m v}$ & $\mathbf{M v}$ & {$[\mathrm{mv}]$} & Mvumbi & Cadavre \\
\hline $\mathbf{1 4}$. & $\mathbf{s}$ & $\mathbf{S}$ & {$[\mathrm{s}]$} & Disala & Pluie fine" \\
\hline $\mathbf{1 5 .}$ & $\mathbf{n z}$ & $\mathbf{N z}$ & {$[\mathrm{nz}]$} & Nzyembu & Reproche \\
\hline $\mathbf{1 6 .}$ & $\mathbf{t s}$ & $\mathbf{T s}$ & {$[\mathrm{ts}]$} & Tsana & Assieds-toi \\
\hline $\mathbf{1 7 .}$ & $\mathbf{n y}$ & $\mathbf{N y}$ & {$[\mathrm{n}]$} & Nyambi & Dieu \\
\hline $\mathbf{1 8 .}$ & $\mathbf{1}$ & $\mathbf{L}$ & {$[\mathrm{L}]$} & Dilolu & Papaye \\
\hline $\mathbf{1 9 .}$ & $\mathbf{r}$ & $\mathbf{R}$ & {$[\mathrm{r}]$} & Burela & Chasse \\
\hline $\mathbf{2 0 .}$ & $\mathbf{w}$ & $\mathbf{W}$ & {$[\mathrm{w}]$} & Bwali & Maladie \\
\hline $\mathbf{2 1 .}$ & $\mathbf{y}$ & $\mathbf{Y}$ & {$[\mathrm{y}]$} & Yotsi & Froid \\
\hline & & & & & \\
\hline
\end{tabular}

\section{UTILISATION DES MAJUSCULES}

Le yipunu utilise la majuscule dans les lettres majuscule dans les mêmes contextes que le français, c'est-à-dire à l'initiale d'une phrase et pour tout type de nom propre: noms de personne, dénomination des localités. Les noms de clans, tribus et de langues s'écrivent aussi avec une lettre majuscule.

Exemples:

\section{Masanga}

Dibala amawanga nuungi. 
Dans les mots composés, chaque constituant du mot commence par une lettre majuscule si c'est un nom propre.

Exemples:

Dibaamba-Kadi (nom de clan)

Duvutu-loongu (nom d'oiseau)

Mavhaanzi-Bakonu (nom d'arbre)

\section{LES JOURS DE LA SEMAINE SE NOTENT EGALEMENT AVEC UNE MAJUSCULE A L'INITIALE.}

Exemples:

Ghari-tsona (mercredi)

Yilumbu-i-Yesu (jeudi)

Tsona (dimanche)

\section{LA PONCTUATION}

\section{LE TRAIT D'UNION}

Tous les termes composés s'écrivent conventionnellement avec un trait d'union en yipunu. Voir les exemples notés plus haut.

\section{LES POINTS:}

Le point final ou d'abréviation (.) - le point-virgule (;) - les deux points (:) - le point d'interrogation (?) - le point d'exclamation (!) - les trois points de suspension (...). Ils sont marqués comme en français et remplissent également les mêmes fonctions.

\section{LES AUTRES SIGNES :}

la virgule $($,$) - les guillemets («») - les parenthèses ($ ) - l'espacement. On les écrit de la même manière avec les mêmes portées sémantiques qu'en français. 


\section{LA DIVISION DU MOT}

\section{LES PREFIXES (NOMINAUX ET VERBAUX), FORMATIFS ET EXTENSIONS}

Les préfixes, les formatifs et les extensions sont joints au radical verbal ou au thème nominal pour former un seul mot. Par contre, les mots composant une phrase sont écris séparément.

Exemple: Amawenda o diimbu (il est parti au village)

\section{LES PREFIXES LOCATIFS AVEC NOMS}

Les préfixes locatifs de classe 16 vha- avec noms s'écrivent en un seul mot: vhatsi (au sol) ou encore vhaghari (au centre).

Les locatifs o- (classe17) et mu- (classe 18) utilisés avec des noms ayant une valeur locative s'écrivent de façon disjonctive.

Exemples: o mbuwaami (chez moi), mu kumu (dans la plaine)

\section{LES PRONOMS POSSESSIFS AVEC LES TERMES DE PARENTE}

Les pronoms possessifs utilisés avec des termes de parenté s'écrivent conjonctivement. Exemples: Tajyaami (mon père), mbatsyaandi (son ami)

\section{LES DEMONSTRATIFS}

Les démonstratifs s'orthographient de façon disjonctive. Exemples: aghu; ghuna; ghunaa; etc. Cependant, les pronoms démonstratifs utilisés avec des noms s'écrivent conjointement: mukwatyaghu (cette machette-ci), murambwaghu (ce piège-ci).

\section{LES ADVERBES}

Les adverbes s'écrivent de façon disjonctive. Car ils constituent une catégorie lexicale autonome.

\section{EXEMPLES:}

Maswasu (vite)

Pweela (beaucoup)

De manière similaire, les adverbes interrogatifs s'écrivent conjointement:

Anyi?; (qui?)

Uka?; (où?)

Ayi?; (quoi?)

Tsye? (comment?) 


\section{APOSTROPHE}

L'apostrophe est utilisée pour indiquer un phénomène d'élision.

\section{LES TONS}

Le yipunu est une langue à tons, c'est-à-dire qu'il utilise les hauteurs mélodiques à des fins distinctives. L'on dénombre cinq contrastes possible aussi bien sur une voyelle brève que sur une voyelle longue: le ton haut, le ton bas, le ton supra haut, le ton montant et le ton descendant. Les indications tonales sont réservées aux études scientifiques dans les universités et autres unités de recherche.

\section{EXEMPLE DE TRANSCRIPTION ORTHOGRAPHIQUE}

Menu, ni maburulu o Masanga, diimbu dyaami Keri-Nzambi. Ivhuunda yi diimbu ayi tegha ayimabanga vha diimbu kaghaami. Dina dyaandi Butamba Bu-Bibogha Théophile. Amabanga na dighumi na baghatsi bana na inombu i baana. $\mathrm{O}$ diimbu bamaama beetu isalu uvhara. Bamabaanda na uresi baka siita na mipala, malaanga, makamba, batiimba nde uranga-urakma. Tumaghwenda o masaku bangebi na bivhuunda byotsu. O masuku meeni tumajannga matuka na banyama ba disimu. 



\section{INDEX THEMATIQUES}

Afrique francophone, 5

Agence de Coopération Culturelle et

Technique (ACCT aujourd'hui

Agence Internationale de la

Francophonie), 27, 56

Alphabet Africa, 40, 41, 43, 50, 75, 76, 104

Alphabet Africain de Référence, 40, 41, 43

Alphabet des idiomes gabonais, 9, 55, 103, 134

Alphabet des langues gabonaises, 55, 61

Alphabet orthographique, 80

Alphabet Phonétique International (API), $16,23,24,41,50,55,57,58,59,60$,

$62,75,76,79,84,104,188,189$

Alphabet Scientifique des Langues du Gabon (ASG), 12, 56, 57, 58, 59, 60, 63, 64, 75, 76, 103, 104, 106, 130, $132,135,136,147,174$

Aménagement linguistique au Gabon, 11

Atlas Linguistique du Gabon (ALGAB), 160

Centre National de la Recherche

Scientifique et Technologique

(CENAREST), 158

CERLAC (Centre d'Etude et de

Recherche sur les Langues d'Afrique

Centrale), 20

CICIBA (Centre International des

Civilisations Bantu), 104, 177

CLAD (Centre de Linguistique

Appliquée de Dakar), 13, 23, 26

CRELL (Centre de recherche en langues et linguistique), 10

Département des langues nationales de l'Institut Pédagogique National, 158

Département des Sciences du Langage (DSL), 10, 86, 148, 158, 159, 209

Direction des Langues Nationales, 10

Ecole Normale Supérieure (ENS), 10, $18,25,158,177$

Etats Généraux du baccalauréat, 61
Fondation Raponda Walker, 10, 162, 175

Francophonie, 6, 8, 56, 104

GRELACO (Groupe de recherche en langues et cultures orales), 10, 26, 173,178

Institut de Recherche en Sciences

Humaines (IRSH), 158

Institut Pédagogique National (IPN), 10

International African Institute (IAI), 41, 50, 58, 59, 60, 75, 76, 79, 103, 104, 177

Journal de la Société des Africanistes, 9, $50,55,105,134$

Laboratoire de Phonétique et Linguistique Africaine (LAPHOLIA), 159

Laboratoire des Sciences Humaines et de la Dynamique du Langage (Lascidyl), $158,159,177$

Laboratoire Universitaire de la Tradition Orale (LUTO), 10, 12, 17, 24, 27, 56, $57,65,66,67,151,177$

Language and Literature Committee of the Christian and Missionary

Alliance (CMA), 118, 127, 128

Méthode Rapidolangue, 105, 209

Ministère de l'Education Nationale, 10, $13,18,26,60,66,80,151,180$

NDAGA (Centre pour l'étude des langues gabonaises), 10, 151

Programme Expérimental Mondial d'Alphabétisation (PEMA), 40

Rapport Final de la Session de Concertation sur l'Orthographe des langues Gabonaises, 130

Recherches linguistiques et l'enseignement des langues gabonaises, 10

Revue Gabonaise des Sciences de l'Homme, 27, 66, 67, 83, 104, 132, 151 
Session de Concertation sur

l'Orthographe des Langues

Gabonaises, 2, 3, 66, 109, 110, 113, $126,140,151,179$

SIL International, 11, 149

Table Ronde sur les Politiques

Linguistiques et l'Enseignement des

Langues Gabonaises, 52
The Practical Orthography of African

Languages, 103

UNESCO, 7, 11, 17, 40, 60

Université Lumière Lyon 2, 27, 66, 103, 131, 132, 133

Université Omar Bongo (UOB), iii, 2 , $10,27,56,65,66,67,79,86,88,133$, 151, 158, 159, 173, 177, 178, 209 


\section{LES CONTRIBUTEURS}

\section{THIERry AfANe OTSAGA}

Linguiste (DUEL, Licence, Maîtrise à l'UOB, Gabon) et lexicographe (Doctorat à l'Université de Stellenbosch, Afrique du Sud). Thierry Afane Otsaga a réalisé une étude phonologique et morphologique du "məká ou Siwa", parler du groupe A70 dans le cadre de son mémoire de Maîtrise. Sa thèse de doctorat a porté sur le rôle du dictionnaire standard bilingue dans la standardisation du fang. Thierry Afane Otsaga s'intéresse particulièrement à l'usage des sciences informatiques pour le développement des langues gabonaises. Après avoir été étudiant conférencier Shell à la Fondation Entreprises en Création (FEC/UOB-Gabon) de 1994 à 1999 et enseignant de langue nationale de 1997 à 1999 au Collège Sainte-Marie et à l'Institution Immaculé Conception, il est actuellement enseignant chercheur au Département des Sciences du Langage de l'Université Omar Bongo (UOB) ainsi que membre permanent de la Chaire UNESCO Interculturalité de Libreville. Il est l'un des membres fondateurs du Gabonese Research E Scientific Discussion Group (GRSDG). Auteur de nombreux articles en lexicographie et en linguistique africaine, Thierry Afane Otsaga est membre de l'Association Africaine pour la Lexicographie (AFRILEX) E-mail: afanotsaga@gmail.com

\section{JACQUES HUBERT}

Diplômé de Cambridge University et de la Royal Society of Arts of London, des Universités de Nantes et d'Angers, professeur de langues (anglais, espagnol et latin) pendant quatorze ans, proviseur du Lycée Catholique Raponda-Walker durant quinze ans, officier dans l'Ordre de l'Education Nationale du Gabon et du Sénégal, Jacques Hubert est le concepteur de la Méthode Rapidolangue. Par rapport à cette dernière, l'auteur fait des emprunts non seulement à des méthodes actuelles d'apprentissage des langues en usage dans divers pays $\mathrm{d}$ 'Afrique mais également aux dernières techniques pédagogiques en la matière. Cette initiative est soutenue par la Fondation Raponda-Walker. Il est l'auteur de plusieurs ouvrages non seulement sur l'enseignement des langues du Gabon et du Cameroun, mais également sur la culture africaine et la liturgie en Afrique. Email : hguerineau@yahoo.fr

\section{Paul Achille Mavoungou}

Maître-Assistant (CAMES) à l'Université Omar Bongo, Paul Achille Mavoungou est linguiste (DUEL, Licence, Maîtrise à l'UOB, Gabon) et lexicographe (Doctorat à l'Université de Stellenbosch en Afrique du Sud). Il a réalisé une étude phonologique et morphologique du "ndafa" (parler du groupe B20) dans le cadre de son mémoire de Maîtrise. Sa thèse de Doctorat a porté sur les critères métalexicographiques pour la confection d'un dictionnaire trilingue yilumbu-anglais-français. Après avoir été tour à tour chercheur affilié au Research Unit for Experimental Phonology University of Stellenbosch (RUEPUS) de 1999 à 2000 et assistant chercheur au Département d'Afrikaans et du Néerlandais à l'Université de Stellenbosch de 1999 à 2001, il est actuellement enseignant-chercheur au Département des Sciences du Langage de l'UOB, membre permanent de la Chaire UNESCO Interculturalité de Libreville 
et egalement Secrétaire Administratif et Financier du Laboratoire Universitaire des Traditions Orales et des Dynamiques Contemporaines. Il est par ailleurs membre de l'Association Africaine pour la Lexicographie (AFRILEX). Il est l'auteur de nombreuses publications en lexicographie et en linguistique africaine. E-mail: moudika2@yahoo.fr

\section{Hugues STEVE NDINGA-Koumba-BinZA}

Spécialiste de phonétique, phonologie et technologies du langage humain, Hugues Steve Ndinga-Koumba-Binza est Attaché de Recherche Post-doctorale au Centre for Text Technology (CTexT) de la North-West University à Potchefstroom (Afrique du Sud) et vacataire à l'Université Omar Bongo. Auparavant à l'Université de Stellenbosch en Afrique du Sud, il a été enseignant invité au Département de Linguistique Générale (2002-2007), vacataire au Département des Langues Etrangères Modernes (2005-2006) et Chercheur au Stellenbosch University Centre for Language and Speech Technology (2003-2009). Sa thèse doctorat sur la durée vocalique en civili fut un travail pionnier des technologies du langage humain et phonologie expérimentale pour les langues gabonaises. Auteur de nombreuses publications, membre de plusieurs organismes professionnels, il siège au Comité Editorial de la revue internationale Lexikos et au Comité d'Evaluation de la Revue des Etudiants en Linguistique du Québec. Membre fondateur de la Stellenbosch University Poetry Society, membre fondateur du Gabonese Research \& Scientific Discussion Group, il a contribué au relancement de la Stellenbosch Post-doctoral Society dont il fut Secrétaire Général (2009-2010). E-mail: 22602569@nwu.ac.za

\section{LEANDRE SERGE SOAMI}

Linguiste (DUEL, Licence, Maîtrise) et lexicographe (Doctorat). Il a réalisé une étude sociolinguistique sur les prénoms à l'Hôpital Pédiatrique d'Owendo (HPO) dans le cadre de son mémoire de Maîtrise. Il a soutenu à l'Université de Stellenbosch, en Afrique du Sud., une thèse portant sur le développement des bases de données lexicographiques représentatives dans les langues gabonaises. Dans le cadre du programme RAPIDOLANGUE, Léandre Serge Soami a été enseignant de langue nationale de 1996 à 2000 au Collège Sainte Marie, à l'Institution Immaculé Conception et au Collège Notre Dame de Quaben. Il est actuellement Attaché de Recherche à l'Institut de Recherche en Sciences Humaines (IRSH-CENAREST) et membre de l'Association Africaine pour la Lexicographie (AFRILEX). Ses articles et autres publication s'inscrivent dans les domaines de la lexicographie et de la sociolinguistique. E-mail: sergesoami@gmail.com 
The language situation of Gabon is given fair treatment and specific issues of alphabet and writing, orthography and standardisation, phonology and graphic representation are discussed and resolved. Standardisation of orthography is also argued for as a way to facilitate the development of dictionaries and the sharing of research data and analyses within the Gabonese languages clusters' domain.

Importantly, this work contributes to the debate on the state of African languages in general, and on Gabonese languages specifically that hitherto have had little fortune in being reduced to a written code. The need for research to empower Gabonese languages through their development is argued.

For policy makers and researchers, this book provides important information for practical consideration regarding the implementation of teaching and writing Gabonese languages.

\section{Dr Andy Chebanne}

The Centre for the Advanced Studies of African Society (CASAS), Cape Town

The validity of this book lies in its comprehensive description of various challenges with the standardisation of Gabonese languages in terms of adopting a common alphabet and phonetics suitable for the transcription of national languages. Most importantly, this book offers new perspectives in addressing the development of Gabonese languages through the appropriation of a unified system of transcription of these languages. The authors of the book suggest a few solutions in the field of linguistics, as well as political decisions which should be made in order to achieve a standardised system of writing Gabonese languages. A particular case study enriches the scientific debate on the development of minority languages and cultures which run the risk of extinction when an established system of writing these languages in terms of alphabet and phonetics is lacking.

\section{Dr Stéphane Serge Ibinga}

Stellenbosch University
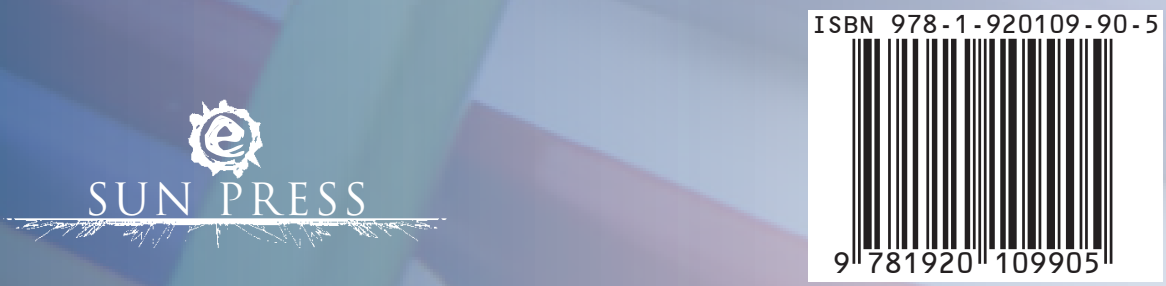

www.sun-e-shop.co.za 